$\mathrm{HL}-90-6$

c. 2

\title{
NUMERICAL MODELING OF KENTUCKY LAKE FLOOD EVENTS
}

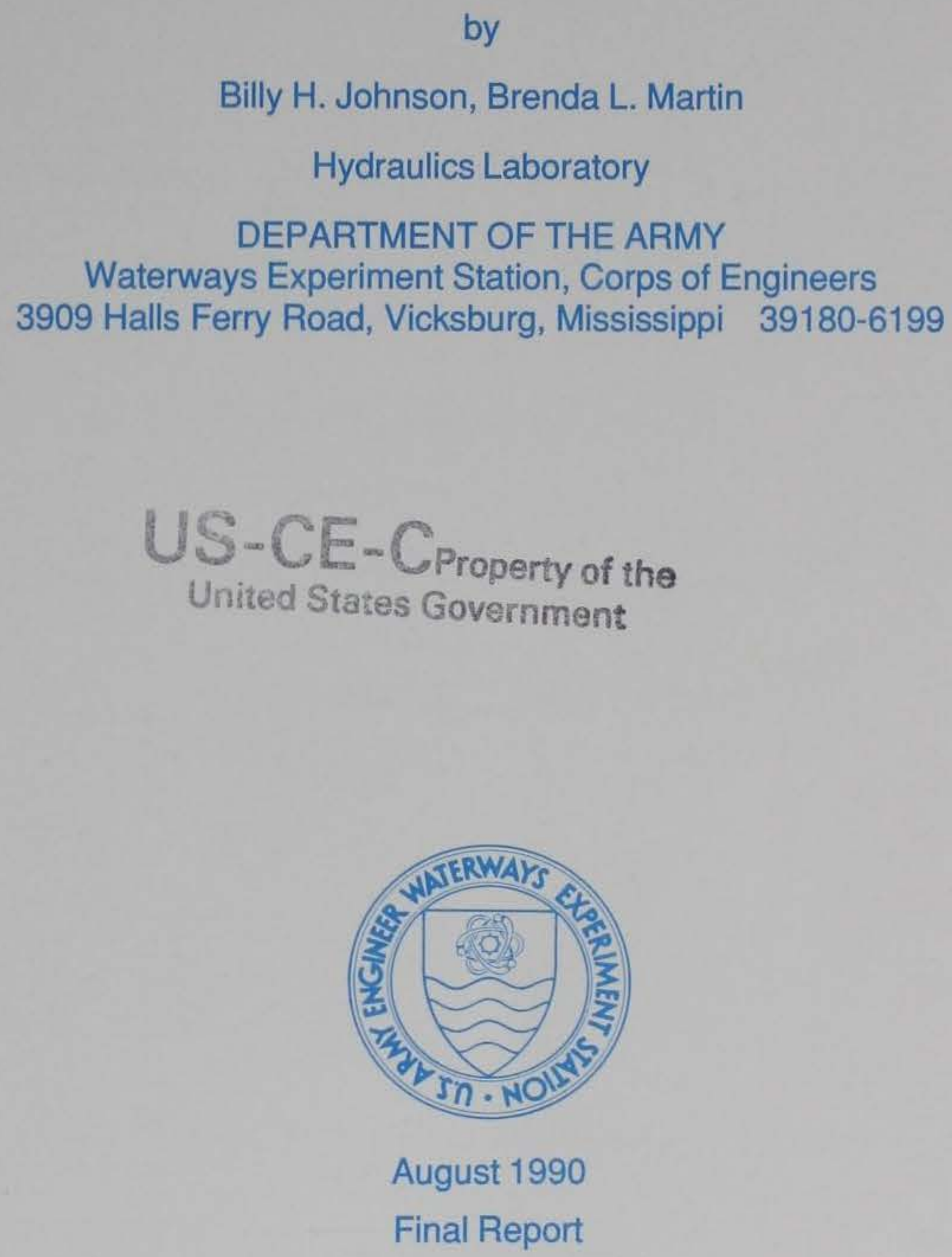

Approved For Public Release; Distribution Unlimited

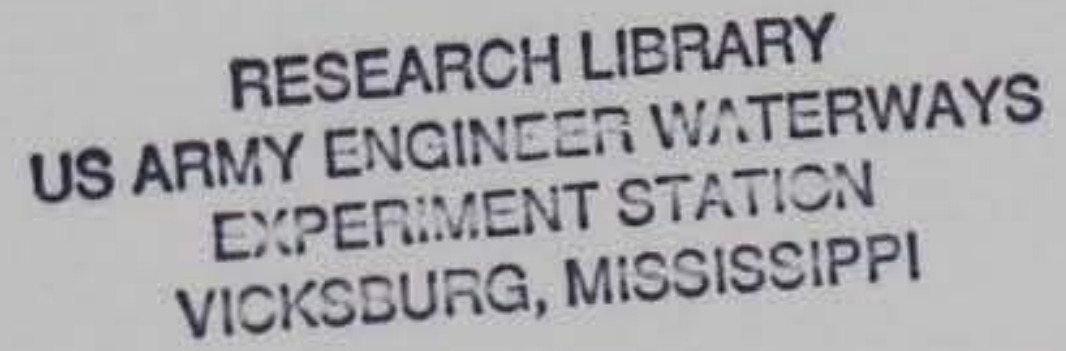

HYDRAULICS 


\title{
DEPARTMENT OF THE ARMY \\ WATERWAYS EXPERIMENT STATION, CORPS OF ENGINEERS \\ P.O. BOX 631 \\ VICKSBURG, MISSISSIPPI 39180-0631
}

REPLY TO

ATTENTION OF

CEWES-HR

14 June 1991

\section{Errata Sheet}

\author{
No. 1 \\ NUMERICAL MODELING OF KENTUCKY LAKE \\ FLOOD EVENTS \\ Miscellaneous Paper HL-90-6 \\ August 1990
}

1. Cover, first figure on left side, downstream end of figure. Change Caruthersville, MO (MRM 847.96) to

MRM 831.72

2. Form 1473, Block 19. ABSTRACT, fifth and seventh lines. Change Caruthersville, MO, to

Mississippi river mile 831.72 ,

3. Page 13, paragraph 24, third line. Change Caruthersville, Mo, to Mississippi river mile (MRM) 831.72,

4. Page 13, paragraph 25, fourth line. Change Caruthersville, MO, to MRM 831.72 .

5. Figure 1, downstream end of figure. Change Caruthersville, Mo (MRM 847.96 ) to

MRM 831.72 
Unclassified

SECURITY CLASSIFICATION OF THIS PAGE

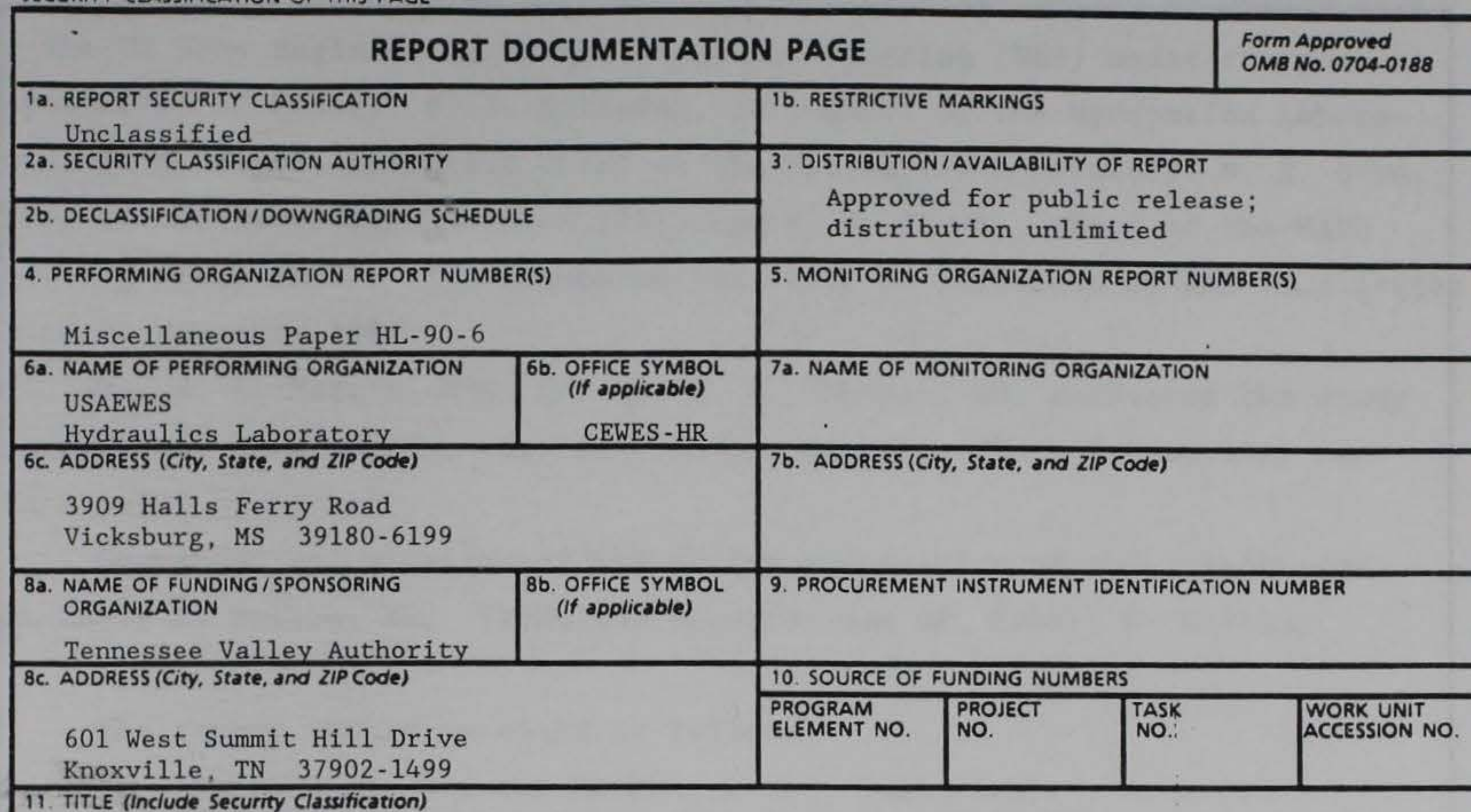

11. TITLE (Include Security Classification)

Numerical Modeling of Kentucky Lake Flood Events

12. PERSONAL AUTHOR(S)

Johnson, Billy $\mathrm{H}$. : Martin, Brenda L

\begin{tabular}{|c|c|c|c|c|}
\hline $\begin{array}{c}\text { 13a. TYPE OF REPORT } \\
\text { Einal Report }\end{array}$ & $\begin{array}{c}\text { 13b. TIME COVERED } \\
\text { FROM }\end{array}$ & $\begin{array}{c}\text { 14. OATE OF REPORT (Year, MONth, Day) } \\
\text { August } 1990\end{array}$ & $\begin{array}{c}\text { 15. PAGE COUNT } \\
133\end{array}$ \\
\hline
\end{tabular}

16. SUPPLEMENTARY NOTATION

Available from National Technical Information Service, 5285 Port Royal Road, Springfield,

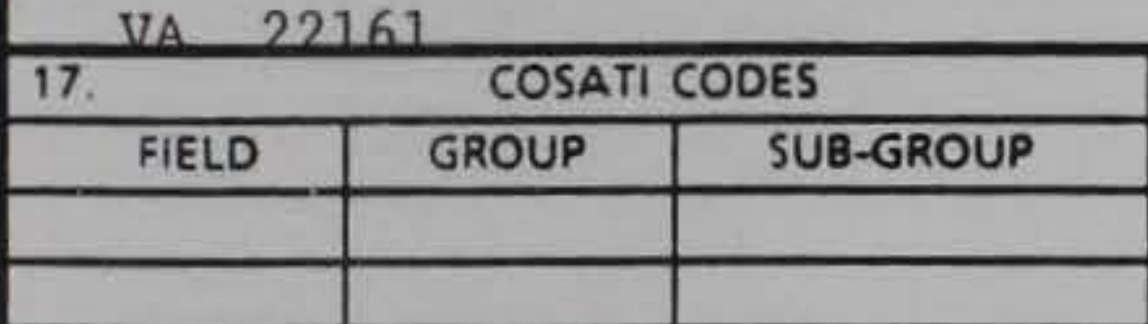

18. SUBJECT TERMS (Continue on reverse if necessary and identify by block number)

Dam break Numerical model

Flood routing Unsteady flow

19. ABSTRACT (Continue on reverse if necessary and identify by block number)

A numerical model called BIRM (B $r a n c h e d ~ I m p l i c i t ~ \underline{R} i v e r ~ M o d e l)$ has been applied assuming that various flood event outflows at Kentucky Dam on the Tennessee River are superimposed on 1937 flow conditions on the Ohio and Mississippi Rivers. Eight different flood events have been routed down the Tennessee, Ohio, and Lower Mississippi Rivers as far as Caruthersville, MO, on the Lower Mississippi. Results in the form of elevation and discharge hydrographs at several key locations plus envelope profiles of the maximum and minimum water-surface elevations from Kentucky Dam to Caruthersville, Mo, are presented for each flood event.

\begin{tabular}{|c|c|c|}
\hline $\begin{array}{l}\text { 20. DISTRIBUTION / AVAILABILITY OF ABSTRACT } \\
\text { Q UNCLASSIFIEDNNLIMITED } \square \text { SAME AS RPT. }\end{array}$ & $\square$ DTIC USERS & $\begin{array}{l}\text { 21. ABSTRACT SECURITY CLASSIFICATION } \\
\text { Unclassified }\end{array}$ \\
\hline 22a. NAME OF RESPONSIBLE INDIVIDUAL & & \begin{tabular}{|l|l|} 
22b. TELEPHONE (InCludo AVed Code) & 22C. OFFICE SYMBOL
\end{tabular} \\
\hline
\end{tabular}




\section{PREFACE}

The work described herein and the preparation of this report were conducted during January to May 1990 for the Tennessee Valley Authority (TVA) by the US Army Engineer Waterways Experiment Station (WES) under the general supervision of Messrs. F. A. Herrmann, Jr., Chief of the Hydraulics Laboratory; R. A. Sager, Assistant Chief of the Hydraulics Laboratory; M. B. Boyd, Chief of the Waterways Division (WD); and M. J. Trawle, Chief of the Math Modeling Group (MMG). TVA requested the study be performed by WES in a letter dated 28 November 1989.

Ms. B. L. Martin, MMG, and Dr. B. H. Johnson, WD, conducted the study and prepared this report. Mr. G. W. Lowe, Manager, Flood Protection, was TVA's point of contact.

Commander and Director of WES during preparation of this report was COL Larry B. Fulton, EN. Technical Director was Dr. Robert W. Whalin.

The report should be cited as follows:

Johnson, B. H., and Martin, B. L. 1990 (Aug). "Numerical Modeling of Kentucky Lake Flood Events," Miscellaneous Paper HL-90-6, US Army Engineer Waterways Experiment Station, Vicksburg, MS. 
PREFACE.

CONVERSION FACTORS, NON-SI TO SI (METRIC)

UNITS OF MEASUREMENT.

Purpose.

Scope.

PART II: DESCRIPTION OF NUMERICAL MODEL.

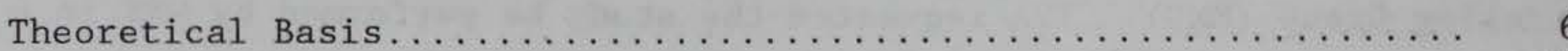

Model Capabilities............................ 10

Limitations. . . . . . . . . . . . 11

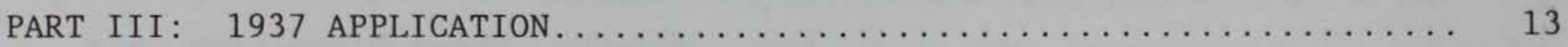

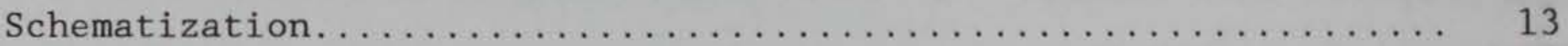

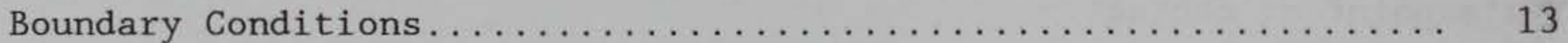

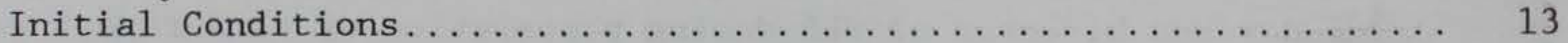

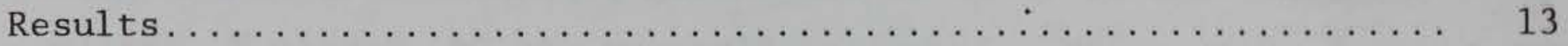

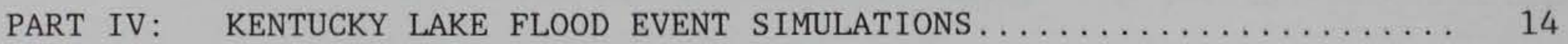

Wilson PMF Dam Break............................. 14

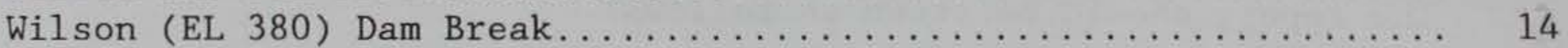

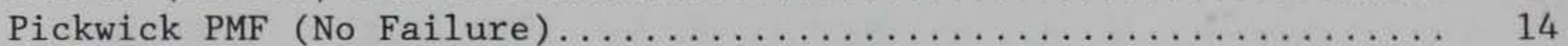

Pickwick PMF Dam Break............................ 15

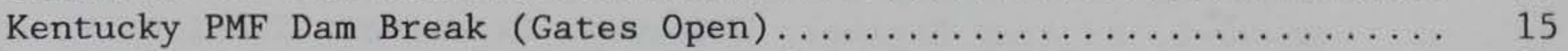

Kentucky PMF Dam Break (Gates Closed) . . . . . . . . . . . . . . 15

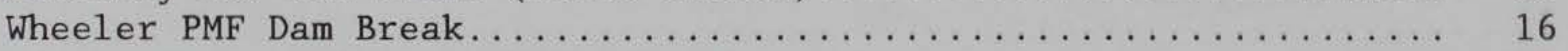

Fort Loudoun-Tellico PMF Dam Break................... 16

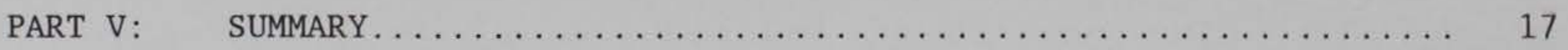

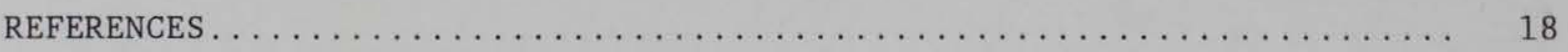

TABLES $1-8$

FIGURES 1-108 
CONVERSION FACTORS, NON-SI TO SI (METRIC)

UNITS OF MEASUREMENT

Non-SI units of measurement used in this report can be converted to SI (metric) units as follows:

Multiply

cubic feet

feet

miles (US statute)
By

0.02831685

0.3048

1.609344
To Obtain

cubic metres

metres

kilometres 
PART I: INTRODUCTION

\section{Purpose}

1. The Tennessee Valley Authority (TVA) is responsible for developing an emergency action plan for Kentucky Dam in the implementation of the Federal Guidelines for Dam Safety. In a letter dated 28 November 1989, the Waterways Experiment Station was requested to route 8 flood events from Kentucky Dam down the Tennessee, Ohio and Lower Mississippi Rivers to Caruthersville, MO. The eight flood events at Kentucky Dam are hypothetical events described to WES purely by the discharge hydrograph from Kentucky Dam furnished by TVA for each event. Results in the form of discharge and elevation hydrographs at key downstream locations as well as envelope profiles of the maximum and minimum water surface elevations will be employed to aid in developing a plan of action.

\section{$\underline{\text { Scope }}$}

2. As a result of previous work for the US Army Engineer, Ohio River Division, WES possesses an open channel unsteady river flow model of the OhioCumberland-Tennessee-Mississippi River system. An early version of that model is called FLOWSED (Johnson, 1982). A later version called BIRM ( r ranched Implicit $\underline{R}$ iver Model) (Johnson 1983) was applied in this study. BIRM was applied a few years ago to route dam breaks from Lake Barkley on the Cumberland River down much of the same river system modeled here (Johnson 1984).

3. In the Lake Barkley dam break study, the basic model was adjusted to match water surface elevations for December 1936 - February 1937 conditions (regulated) as recorded on the Mississippi Basin physical model. The first task implemented in this study was revisiting the basic numerical model to ensure that the 1937 conditions were being reproduced with the correct files. This was required since an extended length of time had passed since the Lake Barkley dam break study and a different computer system was being used.

4. After verifying that the existing numerical model reproduced the 1937 flow conditions, the eight flood events were then routed. In these 
simulations, 1937 inflows were input at Golconda, IL, on the Ohio River and Thebes, MO, on the Upper Mississippi River. As noted above, model discharges at Barkley Dam on the Cumberland River and Kentucky Dam on the Tennessee River for each of the eight flood events were provided by TVA.

5. Following a brief description of the numerical model, results from the 1937 model verification and the eight flood event simulations are presented. 
PART II: DESCRIPTION OF NUMERICAL MODEL

\section{Theoretical Basis}

\section{Basic equations}

6. For the case of one-dimensional open-channel flows within rigid boundaries, the flow behavior can be adequately described by the de SaintVenant partial differential equations of unsteady flow. These equations, which are presented below, are derived by considering the conservation principle for mass and for the momentum of the flow.

Flow Continuity: $\frac{\partial Q}{\partial x}+\frac{\partial A}{\partial t}=q_{\ell}$

Momentum: $\frac{\partial Q}{\partial t}+\mathrm{V} \frac{\partial}{\partial \mathrm{x}}(\beta \mathrm{Q})+\beta \mathrm{V} \frac{\partial \mathrm{Q}}{\partial \mathrm{x}}-\beta \mathrm{V}^{2} \mathrm{~B} \frac{\partial \mathrm{y}}{\partial \mathrm{x}}$

$$
+g A \frac{\partial y}{\partial x}=g A\left(S_{x}-S_{f}-S_{e}\right)+\beta V^{2} A_{x}^{y}
$$

7. Equations 1 and 2 are the set of equations governing the motion of water in open channels in a one-dimensional sense and involve four unknowns; namely, the flow discharge $Q$, the flow depth $y$, the frictional slope $S_{f}$, and an eddy loss term $\mathrm{S}_{\mathrm{e}}$. Other variables such as the lateral inflows and geometry data are expected to be known. To achieve closure of the system, two additional relations are required. These are provided by Manning's equation which relates the friction slope to the flow and channel characteristics,

$$
S_{f}=\frac{n^{2} Q|Q|}{2.25 A^{2} R^{4 / 3}}
$$

and an equation to account for losses due to large-scale eddies formed in the flow at rather abrupt changes in the cross sections along the channel

$$
S_{e}=\frac{K_{e}}{2 g} \frac{\partial v^{2}}{\partial x},
$$

With these additional relations, one can then solve for the basic unknowns Q 
and $y$. Variables in the equations are defined as:

A Total cross-sectional area of channel

$\mathrm{A}_{\mathrm{x}}^{\mathrm{y}} \quad$ Derivative of $\mathrm{A}$ with respect to channel distance at a constant flow depth

B Top width of water surface

g Acceleration due to gravity

$\mathrm{K}_{\mathrm{e}} \quad$ Coefficient in eddy head loss term

$n$ Coefficient in Manning's Equation

Q Flow discharge in cfs

$\mathrm{q}_{\ell} \quad$ Lateral discharge of water

$R \quad$ Hydraulic radius

$\mathrm{S}_{\mathrm{x}} \quad$ Slope of channel bed

$S_{f} \quad$ Friction slope

$\mathrm{S}_{\mathrm{e}} \quad$ Eddy head loss term

$t \quad$ Time

$\mathrm{V} \quad$ Average flow velocity

$\mathrm{x} \quad$ Distance along the channel

$y$ Depth of water in the channel

$z \quad$ Elevation of channel bed

$\beta \quad$ Momentum correction factor

$\partial / \partial t \quad$ Derivative with respect to time

$\partial / \partial \mathrm{x} \quad$ Derivative with respect to channel distance

8. The governing equations do not in general possess analytic solutions. One must therefore rely upon numerical techniques such as finite differences to obtain a solution. The finite difference approximations used in BIRM to express the partial derivatives of a function $\phi$, where $\phi$ represents the dependent variables $Q$ and $y$ are given as

$$
\left.\begin{array}{l}
\frac{\partial \phi}{\partial x} \cong\left(\frac{\phi_{i+1}^{n+1}-\phi_{i}^{n+1}}{\Delta x}\right) \\
\frac{1}{2 \Delta t}\left[\left(\phi_{i}^{n+1}-\phi_{i}^{n}\right)+\left(\phi_{i+1}^{n+1}-\phi_{i+1}^{n}\right)\right]
\end{array}\right\}
$$


where

$$
\Delta \mathrm{x}=\text { spatial computation step }
$$

$\Delta t=$ time computation step

9. Constructing difference equations from the governing differential equations through use of the finite difference approximations presented above results in a linear-implicit finite difference scheme. The difference form of the governing equations written over a computational cell formed by sections $i$ and $i+1$ take the form

$$
\begin{aligned}
& k_{k 1}^{n} Q_{i}^{n+1}+k_{k 2}^{n} y^{n+1}+k_{k 3}^{n} Q_{i+1}^{n+1}+k_{k 4}^{n} Y_{i+1}^{n+1}=E_{k}^{n} \\
& k_{\ell 1}^{n} Q_{i}^{n+1}+k_{\ell 2}^{n} y_{i}^{n+1}+k_{\ell 3}^{n} Q_{i+1}^{n+1}+k_{\ell 4}^{n} Y_{i+1}^{n+1}=E_{\ell}^{n}
\end{aligned}
$$

where $k=2 i, \quad \ell=2 i+1$ and the coefficients $K$ and $E$ are functions of known variables from the previous time line. These equations are then solved using what is commonly called the double sweep algorithm. The interaction between the main river and a tributary being handled in a dynamic fashion as opposed to being treated as lateral inflow is simulated by the following continuity and energy equations:

$$
\begin{aligned}
& Q_{3}=Q_{2}+Q_{1} \\
& Z_{2}+Y_{2}+\frac{v_{2}^{2}}{2 g}=Z_{3}+Y_{3}+\alpha_{2} \frac{v_{3}^{2}}{2 g}+\left(S_{f} \Delta x\right)_{2} \\
& Z_{1}+Y_{1}+\frac{v_{1}^{2}}{2 g}=Z_{3}+Y_{3}+\alpha_{1} \frac{v_{3}^{2}}{2 g}+\left(S_{f} \Delta x\right)_{1}
\end{aligned}
$$

in which $\alpha$ is the energy correction factor, $s_{f} \Delta x$ is the energy head loss and subscripts $1,2,3$, as illustrated below, refer to sections above the confluence on the tributary and main river and below the confluence on the main river. 


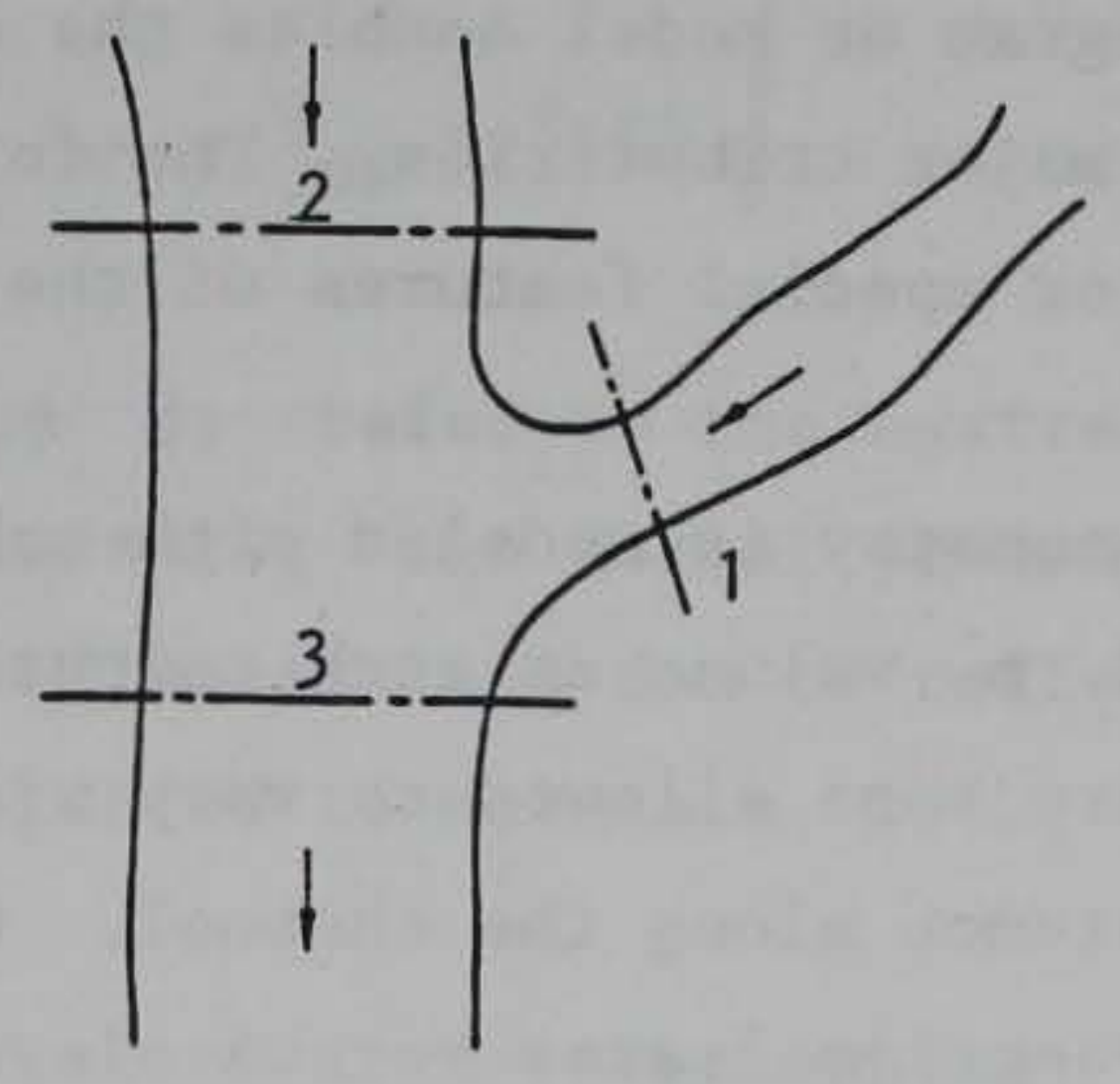

For brevity, details concerning how the junction equations are incorporated into the overall solution scheme using the double sweep algorithm are not presented. Similar computations are discussed by Chen (1973) and Johnson (1982).

\section{Time step restrictions}

10. The solution scheme employed is an implicit finite difference scheme. Unlike explicit schemes, implicit schemes are unconditionally stable. Therefore, the computational time step is not restricted as far as numerical stability is concerned. However, the time step employed does influence the accuracy of the computations.

\section{Boundary computations}

11. Boundary conditions are incorporated directly into the overall solution scheme. This is made possible by writing the boundary conditions in the form of Equation 6. Details are provided by Chen (1973) with additional discussion by Johnson (1982).

\section{Initial conditions}

12. The equations to be solved constitute a hyperbolic system and initial values of the dependent variable, i.e. water surface elevation and discharge must be prescribed to begin the time marching of the solution. A steady-flow profile or perhaps a transient profile from previous computations can be used. The specification of initial conditions is flexible due to the characteristic of hyperbolic equations that the solution becomes independent of initial conditions after a sufficient length of time. 
13. The basic program or model enables the computation of unsteady flow in a main river and its major tributaries. The following discussion describes particular capabilities or special features of the computer code.

\section{Geometric data}

14. The channel geometry is modeled with tables of elevation versus flow area, top width and $n$ values at each computation point along the study reach. The $\mathrm{n}$ values are thus allowed to vary with elevation at a particular node as well as with distance along the channel. Overbank storage is handled by specifying the cross-sectional area versus elevation. The overbank crosssectional areas are then internally converted to plan form areas.

Boundary conditions

15. An upstream boundary condition is prescribed by a flow discharge hydrograph. The hydrograph is coded by specifying the discharge and the number of time steps before a new value will be read. Only those points required to approximate the hydrograph with a sequence of straight lines need. to be entered because linear interpolation is used to develop required values during the computations.

16. At the downstream boundary, a rating curve, a discharge or an elevation hydrograph may be input. An elevation hydrograph is input in exactly the same way as a discharge hydrograph at an upstream boundary. Rating curves are input by representing the rating curve as a sequence of linear segments. Each linear segment is then defined by specifying the discharge and corresponding elevation at the end of the segment.

Local inflow

17. Local inflow may be specified in any computation reach of the study area. Inflows for each specified reach are input in the same manner as the upstream discharge hydrographs.

\section{Locks and dams}

18. To account for the effect of navigation locks and dams, the following equations are utilized: 


$$
\left.\begin{array}{l}
Q_{u s}=Q_{d s} \\
Y_{u s}=f(t)
\end{array}\right\}
$$

where the subscripts us and ds refer to the upstream and downstream sections surrounding the structure. The normal procedure is to input a constant elevation upstream of a lock and dam to reflect the pool elevation that the lock operator is expected to maintain, i.e., $f(t)=$ constant . Levees

19. To handle the effect of levee overtopping, the equation for the discharge over a weir

$$
\mathrm{q}=\mathrm{C} \Delta \mathrm{xH} \mathrm{H}^{3 / 2}
$$

where $\mathrm{H}$ is the height of water over the levee and $\mathrm{C}$ is the discharge coefficient, is invoked to compute lateral outflow from the channel whenever the water surface elevation exceeds the height of the levee. Basic assumptions in the current handling of levees is that the levees do not fail and flow which leaves the channel as a result of levee overtopping is lost from the system. The location and average height of the levee must be specified as input.

Overbanks

20. The lateral inflow $\mathrm{q}_{\ell}$ consists of two components, $\mathrm{q}_{\ell_{1}}$ and $\mathrm{q}_{\ell_{2}}$, induced by the handling of flood plains and tributaries, respectively. For overbank storage, ${ }^{\mathrm{q}} \ell_{1}$ is computed from

$$
\mathrm{q}_{\ell_{1}}=-\frac{\mathrm{A}_{f}}{\Delta \mathrm{x} \Delta t} \Delta \mathrm{h}
$$

where $A_{f}$ is the surface area of the flood plain and $\Delta h$ is the change over a time period $\Delta t$ of the water surface elevation.

\section{Limitations}

21. The model provides a solution of the one-dimensional equations 
describing the motion of water in open channels with irregular cross sections. Therefore, the river reach to be modeled should be reasonably straight with the free surface assumed to be a horizontal line across the section. In addition, the pressure field is assumed to vary in the vertical direction in a hydrostatic manner. Thus, vertical accelerations are negligible, i.e., the flow is gradually varied. Also, the density of the water is homogeneous.

22. The computer model is not a network model since multiple connected systems, i.e., closed loops, cannot be handled. However, it is a junction model since any number of tributaries into the main river are handled in a fully dynamic fashion. In addition, lateral inflows from minor tributaries or ungaged local flows can be accommodated.

23. A current limitation on the physical region is that there can only be one downstream boundary at which a rating curve is employed. In its present form, there is another limitation on the specification of boundary conditions. At an upstream boundary only discharges can be prescribed; whereas, at a downstream boundary either a rating curve, discharges, or water surface elevations may be specified. 


\section{Schematization}

24. A schematization of the study area which is composed of the Ohio River from Golconda, IL, to its junction with the Mississippi River, the Mississippi River from Thebes, IL, to Caruthersville, MO, the Cumberland River from Barkley Dam to its junction with the Ohio River and the Tennessee River from Kentucky Dam to its junction with the Ohio River is presented in Figure 1. The system is discretized with 79 computational points and 7 branches. Key locations are indicated on Figure 1. The spatial step, i.e. $\Delta \mathrm{x}$, is variable but generally varies from one mile to about 5 miles.

\section{Boundary Conditions}

25. Model inflows at Golconda, IL, Barkley Dam, Kentucky Dam and Thebes, MO, were obtained from the Mississippi Basin physical model. These are presented in Figures 2-5. The rating curve presented in Figure 6 was prescribed as the downstream boundary condition at Caruthersville, Mo.

\section{Initial Conditions}

26. Initial conditions were created by holding the inflows at Golconda, Barkley and Kentucky Dams and Thebes constant for 10 days. Model results were then saved and employed as the initial state for the 1937 application as well as the dam break applications discussed in PART IV.

\section{$\underline{\text { Results }}$}

27. Comparisons of computed and recorded elevations from the Mississippi Basin Model at several locations are presented in Figures 7-12. Generally, the computed elevations match the observed elevations to within 0.5 to 1.0 foot. The runs were initiated on 25 December 1936 . Thus day 0 on these plots as well as those presented in PART IV corresponds to 25 December 1936. 
28. Eight different flood events have been modeled. In each case, the 1937 inflow hydrographs at Golconda, IL, and Thebes, MO, presented in Figures 3 and 5, respectively, were prescribed. Discharges from Barkley Dam on the Cumberland River and Kentucky Dam on the Tennessee River for each of the flood event runs were provided by TVA. Initial conditions were those used in the 1937 application discussed in PART III. Results from each of the flood event runs are presented below.

\section{Wilson PMF Dam Break}

29. Figures 13 and 14 show the discharges prescribed from Barkley Lake and Kentucky Lake, respectively, for the Wilson PMF with the postulated failure of Wilson Dam simulation. Time histories of both discharge and elevation computed at several locations along the Ohio and Lower Mississippi Rivers are presented in Figures 15-23. Figure 24 is an envelope profile illustrating both the maximum and minimum elevations computed downstream of Kentucky Dam. These results are presented in tabular form in Table 1.

\section{Wilson (EL 380) Dam Break}

30. Figures 25 and 26 show the discharges prescribed from Barkley Lake and Kentucky Lake, respectively, for the Wilson PMF with the postulated failure of Wilson Dam simulation (Kentucky surcharged to elevation 380). Time histories of both discharge and elevation computed at several locations along the Ohio and Lower Mississippi Rivers are presented in Figures 27-35. Figure 36 is an envelope profile illustrating both the maximum and minimum elevations computed downstream of Kentucky Dam. These results are presented in tabular form in Table 2 .

\section{Pickwick PMF (No Failure)}

31. Figures 37 and 38 show the discharges prescribed from Barkley Lake and Kentucky Lake, respectively, for the Pickwick PMF and no failure of Pickwick Dam simulation. Time histories of both discharge and elevation 
computed at several locations along the Ohio and Lower Mississippi Rivers are presented in Figures 39-47. Figure 48 is an envelope profile illustrating both the maximum and minimum elevations computed downstream of Kentucky Dam. These results are presented in tabular form in Table 3.

\section{Pickwick PMF Dam Break}

32. Figures 49 and 50 show the discharges prescribed from Barkley Lake and Kentucky Lake, respectively, for the Pickwick PMF with the postulated failure of Pickwick Dam simulation. Time histories of both discharge and elevation computed at several locations along the Ohio and Lower Mississippi Rivers are presented in Figures 51-59. Figure 60 is an envelope profile illustrating both the maximum and minimum elevations computed downstream of Kentucky Dam. These results are presented in tabular form in Table 4.

\section{Kentucky PMF Dam Break (Gates Open)}

33. Figures 61 and 62 show the discharges prescribed from Barkley Lake and Kentucky Lake, respectively, for the Kentucky PMF with the postulated failure of Kentucky Dam simulation (with gates remaining open at Kentucky following failure). Time histories of both discharge and elevation computed at several locations along the Ohio and Lower Mississippi Rivers are presented in Figures 63-71. Figure 72 is an envelope profile illustrating both the maximum and minimum elevations computed downstream of Kentucky Dam. These results are presented in tabular form in Table 5.

\section{Kentucky PMF Dam Break (Gates Closed)}

34. Figures 73 and 74 show the discharges prescribed from Barkley Lake and Kentucky Lake, respectively, for the Kentucky PMF with the postulated failure of Kentucky Dam simulation (with gates closed at Kentucky following failure). Time histories of both discharge and elevation computed at several locations along the Ohio and Lower Mississippi Rivers are presented in Figures 75-83. Figure 84 is an envelope profile illustrating both the maximum and minimum elevations computed downstream of Kentucky Dam. These results are presented in tabular form in Table 6 . 


\section{Wheeler PMF Dam Break}

35. Figures 85 and 86 show the discharges prescribed from Barkley Lake and Kentucky Lake, respectively, for the Wheeler PMF with the postulated failure of Wheeler Dam simulation. Time histories of both discharge and elevation computed at several locations along the Ohio and Lower Mississippi Rivers are presented in Figures 87-95. Figure 96 is an envelope profile illustrating both the maximum and minimum elevations computed downstream of Kentucky Dam. These results are presented in tabular form in Table 7.

\section{Fort Loudoun-Tellico PMF Dam Break}

36. Figures 97 and 98 show the discharges prescribed from Barkley Lake and Kentucky Lake, respectively, for the Fort Loudoun-Tellico PMF with the postulated failure of Fort Loudoun-Tellico simulation. Time histories of both discharge and elevation computed at several locations along the Ohio and Lower Mississippi Rivers are presented in Figures 99-107. Figure 108 is an envelope profile illustrating both the maximum and minimum elevations computed downstream of Kentucky Dam. These results are presented in tabular form in Table 8 . 


\section{PART V: SUMMARY}

37. A 1D numerical model called BIRM for computing unsteady flow in open channels has been applied to route several flood events down the Tennessee, Ohio and Lower Mississippi Rivers. These floods include PMF events and assumed dam failures at Kentucky Dam and other upstream dams on the Tennessee River. Results in the form of discharge and elevation hydrographs at several locations along the Ohio and Lower Mississippi Rivers are presented for each routed flood event along with profiles of the maximum and minimum water surface elevation downstream of Kentucky Dam. These results, will be used by TVA in developing an emergency action plan for Kentucky Dam. 


\section{REFERENCES}

Chen, Y. H. 1973. "Mathematical Modeling of Water and Sediment Routing in Natural Channels," Ph.D. Dissertation at Colorado State University, Fort Collins, CO.

Johnson, B. H. 1982. "Development of a Numerical Modeling Capability for the Computation of Unsteady Flow on the Ohio River and Its Major Tributaries," Technical Report HL-82-20, US Army Engineer Waterways Experiment Station, Vicksburg, MS.

\footnotetext{
1983. "User's Guide for Branched Implicit River Model (BIRM) with Application to the Lower Mississippi river," Unpublished Internal Report in Hydraulics Laboratory, US Army Engineer Waterways Experiment Station, Vicksburg, MS.

. 1984. "Computation of Unsteady Flow on the Lower Cumberland, Lower Tennessee, Ohio and Mississippi Rivers as a Result of Dam Failure at Barkley and Kentucky Dams," Unpublished Internal Report in Hydraulics Laboratory, US Army Engineer Waterways Experiment Station, Vicksburg, MS.
} 
Table 1

\section{Envelope Profile Downstream of Kentucky Dam for Wilson PMF Dambreak}

\begin{tabular}{|c|c|c|}
\hline $\begin{array}{l}\text { Maximum } \\
\text { Elevation } \\
(\mathrm{Ft}) \\
\end{array}$ & $\begin{array}{l}\text { Minimum } \\
\text { Elevation } \\
\text { (Ft) } \\
\end{array}$ & $\begin{array}{c}\text { Distance Below } \\
\text { Kentucky Dam } \\
\text { (Miles) } \\
\end{array}$ \\
\hline 355.31 & 303.44 & 0.00 \\
\hline 355.19 & 303.06 & 3.82 \\
\hline 354.99 & 302.56 & 7.41 \\
\hline 354.80 & 302.09 & 11.00 \\
\hline 354.67 & 301.73 & 14.54 \\
\hline 354.46 & 301.37 & 18.03 \\
\hline 353.96 & 299.84 & 19.88 \\
\hline 353.48 & 299.80 & 22.58 \\
\hline 352.87 & 299.41 & 23.53 \\
\hline 352.47 & 299.08 & 24.28 \\
\hline 352.05 & 298.76 & 24.98 \\
\hline 351.68 & 298.49 & 28.06 \\
\hline 348.89 & 296.80 & 33.44 \\
\hline 346.50 & 295.64 & 38.71 \\
\hline 344.19 & 294.31 & 44.04 \\
\hline 342.29 & 293.15 & 49.36 \\
\hline 340.54 & 292.20 & 54.69 \\
\hline 338.57 & 290.52 & 59.46 \\
\hline 337.41 & 289.01 & 63.79 \\
\hline 336.42 & 287.94 & 67.10 \\
\hline 335.86 & 287.48 & 70.65 \\
\hline 333.08 & 286.13 & 75.54 \\
\hline 330.71 & 284.65 & 80.48 \\
\hline 328.45 & 283.14 & 86.99 \\
\hline 325.41 & 280.79 & 94.48 \\
\hline 323.06 & 278.42 & 100.45 \\
\hline 321.19 & 276.45 & 105.44 \\
\hline 319.08 & 274.25 & 111.29 \\
\hline 317.00 & 271.36 & 116.59 \\
\hline 315.62 & 269.70 & 120.64 \\
\hline 314.05 & 268.75 & 124.82 \\
\hline 311.80 & 266.05 & 127.89 \\
\hline 310.69 & 264.69 & 130.93 \\
\hline 308.80 & 263.19 & 134.82 \\
\hline 306.86 & 262.18 & 138.38 \\
\hline 305.32 & 261.52 & 142.60 \\
\hline 303.48 & 261.17 & 147.03 \\
\hline 301.56 & 260.17 & 150.94 \\
\hline 299.12 & 257.36 & 155.11 \\
\hline 296.93 & 255.32 & 159.37 \\
\hline 294.36 & 254.64 & 163.53 \\
\hline 293.00 & 254.33 & 167.83 \\
\hline 290.09 & 252.35 & 172.04 \\
\hline 285.23 & 249.54 & 176.48 \\
\hline
\end{tabular}


Table 1 (Continued)

\begin{tabular}{|c|c|c|}
\hline $\begin{array}{l}\text { Maximum } \\
\text { Elevation } \\
\text { (Ft) } \\
\end{array}$ & $\begin{array}{c}\text { Minimum } \\
\text { Elevation } \\
\text { (Ft) } \\
\end{array}$ & $\begin{array}{c}\text { Distance Below } \\
\text { Kentucky Dam } \\
\text { (Miles) } \\
\end{array}$ \\
\hline 280.11 & 247.73 & 180.55 \\
\hline 278.18 & 247.09 & 184.16 \\
\hline 275.43 & 246.62 & 188.21 \\
\hline 272.96 & 245.97 & 192.35 \\
\hline
\end{tabular}


Table 2

Envelope Profile downstream of Kentucky Dam for Wilson PMF (Elev 380) Dambreak

\begin{tabular}{|c|c|c|}
\hline $\begin{array}{l}\text { Maximum } \\
\text { Elevation } \\
\text { (Ft) } \\
\end{array}$ & $\begin{array}{l}\text { Minimum } \\
\text { Elevation } \\
\text { (Ft) } \\
\end{array}$ & $\begin{array}{c}\text { Distance Below } \\
\text { Kentucky Dam } \\
\text { (Miles) } \\
\end{array}$ \\
\hline 353.19 & 303.69 & 0.00 \\
\hline 353.09 & 303.30 & 3.82 \\
\hline 352.92 & 302.77 & 7.41 \\
\hline 352.75 & 302.27 & 11.00 \\
\hline 352.63 & 301.88 & 14.54 \\
\hline 352.44 & 301.39 & 18.03 \\
\hline 352.02 & 299.84 & 19.88 \\
\hline 351.55 & 299.81 & 22.58 \\
\hline 350.96 & 299.41 & 23.53 \\
\hline 350.56 & 299.08 & 24.28 \\
\hline 350.16 & 298.76 & 24.98 \\
\hline 349.80 & 298.49 & 28.06 \\
\hline 347.23 & 296.80 & 33.44 \\
\hline 345.06 & 295.64 & 38.71 \\
\hline 342.96 & 294.31 & 44.04 \\
\hline 341.22 & 293.16 & 49.36 \\
\hline 339.62 & 292.22 & 54.69 \\
\hline 337.81 & 290.57 & 59.46 \\
\hline 336.72 & 289.08 & 63.79 \\
\hline 335.80 & 288.01 & 67.10 \\
\hline 335.24 & 287.54 & 70.65 \\
\hline 332.48 & 286.19 & 75.54 \\
\hline 330.13 & 284.71 & 80.48 \\
\hline 327.89 & 283.19 & 86.99 \\
\hline 324.85 & 280.82 & 94.48 \\
\hline 322.51 & 278.42 & 100.45 \\
\hline 320.65 & 276.45 & 105.44 \\
\hline 318.55 & 274.25 & 111.29 \\
\hline 316.46 & 271.36 & 116.59 \\
\hline 315.09 & 269.70 & 120.64 \\
\hline 313.52 & 268.75 & 124.82 \\
\hline 311.27 & 266.05 & 127.89 \\
\hline 310.16 & 264.69 & 130.93 \\
\hline 308.28 & 263.19 & 134.82 \\
\hline 306.34 & 262.18 & 138.38 \\
\hline 304.80 & 261.52 & 142.60 \\
\hline 302.98 & 261.17 & 147.03 \\
\hline 301.08 & 260.17 & 150.94 \\
\hline 298.65 & 257.36 & 155.11 \\
\hline 296.47 & 255.32 & 159.37 \\
\hline 293.93 & 254.64 & 163.53 \\
\hline 292.58 & 254.33 & 167.83 \\
\hline
\end{tabular}


Table 2 (Continued)

\begin{tabular}{ccc}
\hline $\begin{array}{c}\text { Maximum } \\
\text { Elevation } \\
\text { (Ft) }\end{array}$ & $\begin{array}{c}\text { Minimum } \\
\text { Elevation } \\
(\mathrm{Ft})\end{array}$ & $\begin{array}{c}\text { Distance Below } \\
\text { Kentucky Dam } \\
\text { (Miles) }\end{array}$ \\
\cline { 1 - 2 } 289.70 & 252.35 & 172.04 \\
284.86 & 249.54 & 176.48 \\
279.76 & 247.73 & 180.55 \\
277.85 & 247.09 & 184.16 \\
275.15 & 246.62 & 188.21 \\
272.72 & 245.97 & 192.35
\end{tabular}


Table 3

Envelope Profile Downstream of Kentucky Dam for Pickwick

PMF (no failure)

\begin{tabular}{|c|c|c|}
\hline $\begin{array}{l}\text { Maximum } \\
\text { Elevation } \\
\text { (Ft) } \\
\end{array}$ & $\begin{array}{l}\text { Minimum } \\
\text { Elevation } \\
(\mathrm{Ft}) \\
\end{array}$ & $\begin{array}{c}\text { Distance Below } \\
\text { Kentucky Dam } \\
\text { (Miles) } \\
\end{array}$ \\
\hline 354.01 & 303.69 & 0.00 \\
\hline 353.90 & 303.30 & 3.82 \\
\hline 353.70 & 302.77 & 7.41 \\
\hline 353.52 & 302.27 & 11.00 \\
\hline 353.39 & 301.88 & 14.54 \\
\hline 353.18 & 301.39 & 18.03 \\
\hline 352.71 & 299.84 & 19.88 \\
\hline 352.24 & 299.81 & 22.58 \\
\hline 351.64 & 299.41 & 23.53 \\
\hline 351.24 & 299.08 & 24.28 \\
\hline 350.82 & 298.76 & 24.98 \\
\hline 350.46 & 298.49 & 28.06 \\
\hline 347.69 & 296.80 & 33.44 \\
\hline 345.33 & 295.64 & 38.71 \\
\hline 343.05 & 294.31 & 44.04 \\
\hline 341.17 & 293.16 & 49.36 \\
\hline 339.44 & 292.22 & 54.69 \\
\hline 337.49 & 290.57 & 59.46 \\
\hline 336.34 & 289.08 & 63.79 \\
\hline 335.37 & 288.01 & 67.10 \\
\hline 334.81 & 287.54 & 70.65 \\
\hline 332.04 & 286.19 & 75.54 \\
\hline 329.69 & 284.71 & 80.48 \\
\hline 327.45 & 283.19 & 86.99 \\
\hline 324.43 & 280.82 & 94.48 \\
\hline 322.09 & 278.42 & 100.45 \\
\hline 320.24 & 276.45 & 105.44 \\
\hline 318.14 & 274.25 & 111.29 \\
\hline 316.06 & 271.36 & 116.59 \\
\hline 314.69 & 269.70 & 120.64 \\
\hline 313.12 & 268.75 & 124.82 \\
\hline 310.87 & 266.05 & 127.89 \\
\hline 309.77 & 264.69 & 130.93 \\
\hline 307.88 & 263.19 & 134.82 \\
\hline 305.95 & 262.18 & 138.38 \\
\hline 304.42 & 261.52 & 142.60 \\
\hline 302.61 & 261.17 & 147.03 \\
\hline 300.72 & 260.17 & 150.94 \\
\hline 298.30 & 257.36 & 155.11 \\
\hline 296.13 & 255.32 & 159.37 \\
\hline 293.62 & 254.64 & 163.53 \\
\hline 292.27 & 254.33 & 167.83 \\
\hline
\end{tabular}

(Continued) 
Table 3 (Continued)

\begin{tabular}{ccc}
\hline $\begin{array}{c}\text { Maximum } \\
\text { Elevation } \\
(\mathrm{Ft})\end{array}$ & $\begin{array}{c}\text { Minimum } \\
\text { Elevation } \\
(\mathrm{Ft})\end{array}$ & $\begin{array}{c}\text { Distance Below } \\
\text { Kentucky Dam } \\
\text { (Miles) }\end{array}$ \\
\cline { 1 - 2 } 289.41 & 252.35 & $\begin{array}{c}172.04 \\
284.58\end{array}$ \\
279.51 & 249.54 & 176.48 \\
277.61 & 247.73 & 180.55 \\
274.95 & 247.09 & 184.16 \\
272.54 & 246.62 & 188.21 \\
& 245.97 & 192.35
\end{tabular}


Table 4

Envelope Profile Downstream of Kentucky Dam for

Pickwick PMF Dambreak

\begin{tabular}{|c|c|c|}
\hline $\begin{array}{l}\text { Maximum } \\
\text { Elevation } \\
\text { (Ft) } \\
\end{array}$ & $\begin{array}{c}\text { Minimum } \\
\text { Elevation } \\
\text { (Ft) } \\
\end{array}$ & $\begin{array}{c}\text { Distance Below } \\
\text { Kentucky Dam } \\
\text { (Miles) } \\
\end{array}$ \\
\hline 354.39 & 303.69 & 0.00 \\
\hline 354.27 & 303.30 & 3.82 \\
\hline 354.08 & 302.77 & 7.41 \\
\hline 353.91 & 302.27 & 11.00 \\
\hline 353.79 & 301.88 & 14.54 \\
\hline 353.59 & 301.39 & 18.03 \\
\hline 353.13 & 299.84 & 19.88 \\
\hline 352.66 & 299.81 & 22.58 \\
\hline 352.06 & 299.41 & 23.53 \\
\hline 351.66 & 299.08 & 24.28 \\
\hline 351.24 & 298.76 & 24.98 \\
\hline 350.88 & 298.49 & 28.06 \\
\hline 348.12 & 296.80 & 33.44 \\
\hline 345.76 & 295.64 & 38.71 \\
\hline 343.48 & 294.31 & 44.04 \\
\hline 341.61 & 293.16 & 49.36 \\
\hline 339.88 & 292.22 & 54.69 \\
\hline 337.93 & 290.57 & 59.46 \\
\hline 336.78 & 289.08 & 63.79 \\
\hline 335.80 & 288.01 & 67.10 \\
\hline 335.24 & 287.54 & 70.65 \\
\hline 332.47 & 286.19 & 75.54 \\
\hline 330.12 & 284.71 & 80.48 \\
\hline 327.87 & 283.19 & 86.99 \\
\hline 324.84 & 280.82 & 94.48 \\
\hline 322.51 & 278.42 & 100.45 \\
\hline 320.65 & 276.45 & 105.44 \\
\hline 318.54 & 274.25 & 111.29 \\
\hline 316.46 & 271.36 & 116.59 \\
\hline 315.09 & 269.70 & 120.64 \\
\hline 313.52 & 268.75 & 124.82 \\
\hline 311.27 & 266.05 & 127.89 \\
\hline 310.16 & 264.69 & 130.93 \\
\hline 308.27 & 263.19 & 134.82 \\
\hline 306.34 & 262.18 & 138.38 \\
\hline 304.80 & 261.52 & 142.60 \\
\hline 302.98 & 261.17 & 147.03 \\
\hline 301.08 & 260.17 & 150.94 \\
\hline 298.65 & 257.36 & 155.11 \\
\hline 296.47 & 255.32 & 159.37 \\
\hline 293.94 & 254.64 & 163.53 \\
\hline 292.59 & 254.33 & 167.83 \\
\hline
\end{tabular}


Table 4 (Continued)

\begin{tabular}{ccc}
\hline $\begin{array}{c}\text { Maximum } \begin{array}{c}\text { Elevation } \\
(\text { Ft) }\end{array} \\
\text { 289.70 }\end{array}$ & $\begin{array}{c}\text { Minimum } \\
\text { Elevation } \\
(\mathrm{Ft})\end{array}$ & $\begin{array}{c}\text { Distance Below } \\
\text { Kentucky Dam } \\
\text { (Miles) }\end{array}$ \\
\cline { 2 - 2 } 284.86 & 252.35 & 172.04 \\
279.77 & 249.54 & 176.48 \\
277.85 & 247.73 & 180.55 \\
275.15 & 247.09 & 184.16 \\
272.72 & 246.62 & 188.21 \\
& 245.97 & 192.35
\end{tabular}


Table 5

Envelope Profile Downstream of Kentucky Dam

for Kentucky PMF Dambreak (Gates Open)

\begin{tabular}{|c|c|c|}
\hline $\begin{array}{l}\text { Maximum } \\
\text { Elevation } \\
\text { (Ft) } \\
\end{array}$ & $\begin{array}{l}\text { Minimum } \\
\text { Elevation } \\
(\mathrm{Ft}) \\
\end{array}$ & $\begin{array}{c}\text { Distance Below } \\
\text { Kentucky Dam } \\
\text { (Miles) } \\
\end{array}$ \\
\hline 361.74 & 303.69 & 0.00 \\
\hline 361.59 & 303.30 & 3.82 \\
\hline 361.38 & 302.77 & 7.41 \\
\hline 361.21 & 302.27 & 11.00 \\
\hline 361.09 & 301.88 & 14.54 \\
\hline 360.87 & 301.39 & 18.03 \\
\hline 360.28 & 299.84 & 19.88 \\
\hline 359.77 & 299.81 & 22.58 \\
\hline 359.13 & 299.41 & 23.53 \\
\hline 358.70 & 299.08 & 24.28 \\
\hline 358.26 & 298.76 & 24.98 \\
\hline 357.86 & 298.49 & 28.06 \\
\hline 354.89 & 296.80 & 33.44 \\
\hline 352.36 & 295.64 & 38.71 \\
\hline 349.94 & 294.31 & 44.04 \\
\hline 347.96 & 293.16 & 49.36 \\
\hline 346.13 & 292.22 & 54.69 \\
\hline 344.10 & 290.57 & 59.46 \\
\hline 342.93 & 289.08 & 63.79 \\
\hline 341.95 & 288.01 & 67.10 \\
\hline 341.36 & 287.54 & 70.65 \\
\hline 338.56 & 286.19 & 75.54 \\
\hline 336.16 & 284.71 & 80.48 \\
\hline 333.84 & 283.19 & 86.99 \\
\hline 330.68 & 280.82 & 94.48 \\
\hline 328.28 & 278.42 & 100.45 \\
\hline 326.36 & 276.45 & 105.44 \\
\hline 324.17 & 274.25 & 111.29 \\
\hline 322.09 & 271.36 & 116.59 \\
\hline 320.69 & 269.70 & 120.64 \\
\hline 319.13 & 268.75 & 124.82 \\
\hline 316.87 & 266.05 & 127.89 \\
\hline 315.69 & 264.69 & 130.93 \\
\hline 313.76 & 263.19 & 134.82 \\
\hline 311.80 & 262.18 & 138.38 \\
\hline 310.20 & 261.52 & 142.60 \\
\hline 308.17 & 261.17 & 147.03 \\
\hline 306.13 & 260.17 & 150.94 \\
\hline 303.61 & 257.36 & 155.11 \\
\hline 301.33 & 255.32 & 159.37 \\
\hline 298.45 & 254.64 & 163.53 \\
\hline 297.02 & 254.33 & 167.83 \\
\hline
\end{tabular}


Table 5 (Continued)

\begin{tabular}{|c|c|c|}
\hline $\begin{array}{l}\text { Maximum } \\
\text { Elevation } \\
(\mathrm{Ft}) \\
\end{array}$ & $\begin{array}{l}\text { Minimum } \\
\text { Elevation } \\
\text { (Ft) } \\
\end{array}$ & $\begin{array}{c}\text { Distance Below } \\
\text { Kentucky Dam } \\
\text { (Miles) } \\
\end{array}$ \\
\hline 293.88 & 252.35 & 172.04 \\
\hline 288.85 & 249.54 & 176.48 \\
\hline 283.54 & 247.73 & 180.55 \\
\hline 281.39 & 247.09 & 184.16 \\
\hline 278.21 & 246.62 & 188.21 \\
\hline 275.41 & 245.97 & 192.35 \\
\hline
\end{tabular}


Table 6

Envelope Profile Downstream of Kentucky Dam for

Kentucky PMF Dambreak (Gates Closed)

\begin{tabular}{|c|c|c|}
\hline $\begin{array}{l}\text { Maximum } \\
\text { Elevation } \\
\text { (Ft) } \\
\end{array}$ & $\begin{array}{l}\text { Minimum } \\
\text { Elevation } \\
\text { (Ft) } \\
\end{array}$ & $\begin{array}{c}\text { Distance Below } \\
\text { Kentucky Dam } \\
\text { (Miles) } \\
\end{array}$ \\
\hline 358.00 & 303.69 & 0.00 \\
\hline 357.88 & 303.30 & 3.82 \\
\hline 357.69 & 302.77 & 7.41 \\
\hline 357.52 & 302.27 & 11.00 \\
\hline 357.40 & 301.88 & 14.54 \\
\hline 357.19 & 301.39 & 18.03 \\
\hline 356.68 & 299.84 & 19.88 \\
\hline 356.19 & 299.81 & 22.58 \\
\hline 355.57 & 299.41 & 23.53 \\
\hline 355.16 & 299.08 & 24.28 \\
\hline 354.74 & 298.76 & 24.98 \\
\hline 354.37 & 298.49 & 28.06 \\
\hline 351.54 & 296.80 & 33.44 \\
\hline 349.12 & 295.64 & 38.71 \\
\hline 346.79 & 294.31 & 44.04 \\
\hline 344.89 & 293.16 & 49.36 \\
\hline 343.13 & 292.22 & 54.69 \\
\hline 341.16 & 290.57 & 59.46 \\
\hline 340.01 & 289.08 & 63.79 \\
\hline 339.05 & 288.01 & 67.10 \\
\hline 338.47 & 287.54 & 70.65 \\
\hline 335.70 & 286.19 & 75.54 \\
\hline 333.33 & 284.71 & 80.48 \\
\hline 331.04 & 283.19 & 86.99 \\
\hline 327.95 & 280.82 & 94.48 \\
\hline 325.58 & 278.42 & 100.45 \\
\hline 323.69 & 276.45 & 105.44 \\
\hline 321.54 & 274.25 & 111.29 \\
\hline 319.46 & 271.36 & 116.59 \\
\hline 318.07 & 269.70 & 120.64 \\
\hline 316.51 & 268.75 & 124.82 \\
\hline 314.25 & 266.05 & 127.89 \\
\hline 313.11 & 264.69 & 130.93 \\
\hline 311.20 & 263.19 & 134.82 \\
\hline 309.25 & 262.18 & 138.38 \\
\hline 307.68 & 261.52 & 142.60 \\
\hline 305.75 & 261.17 & 147.03 \\
\hline 303.77 & 260.17 & 150.94 \\
\hline 301.29 & 257.36 & 155.11 \\
\hline 299.06 & 255.32 & 159.37 \\
\hline 296.34 & 254.64 & 163.53 \\
\hline
\end{tabular}


Table 6 (Continued)

\begin{tabular}{ccc}
\hline $\begin{array}{c}\text { Maximum } \begin{array}{c}\text { Elevation } \\
(\mathrm{Ft})\end{array} \\
294.95\end{array}$ & $\begin{array}{c}\text { Minimum } \\
\text { Elevation } \\
(\mathrm{Ft})\end{array}$ & $\begin{array}{c}\text { Distance Below } \\
\text { Kentucky Dam } \\
\text { (Miles) }\end{array}$ \\
\cline { 2 - 3 } 291.92 & 254.33 & 167.83 \\
286.97 & 252.35 & 172.04 \\
281.76 & 249.54 & 176.48 \\
279.72 & 247.73 & 180.55 \\
276.76 & 247.09 & 184.16 \\
274.13 & 246.62 & 188.21 \\
& 245.97 & 192.35
\end{tabular}


Table 7

Envelope Profile Downstream of Kentucky Dam for

Wheeler PMF Dambreak

\begin{tabular}{|c|c|c|}
\hline $\begin{array}{l}\text { Maximum } \\
\text { Elevation } \\
(\mathrm{Ft}) \\
\end{array}$ & $\begin{array}{l}\text { Minimum } \\
\text { Elevation } \\
\text { (Ft) } \\
\end{array}$ & $\begin{array}{c}\text { Distance Below } \\
\text { Kentucky Dam } \\
\text { (Miles) } \\
\end{array}$ \\
\hline 354.36 & 300.75 & 0.00 \\
\hline 354.23 & 300.66 & 3.82 \\
\hline 354.02 & 300.55 & 7.41 \\
\hline 353.82 & 300.44 & 11.00 \\
\hline 353.68 & 300.37 & 14.54 \\
\hline 353.46 & 300.30 & 18.03 \\
\hline 352.95 & 299.83 & 19.88 \\
\hline 352.48 & 299.79 & 22.58 \\
\hline 351.87 & 299.40 & 23.53 \\
\hline 351.47 & 299.07 & 24.28 \\
\hline 351.05 & 298.75 & 24.98 \\
\hline 350.68 & 298.49 & 28.06 \\
\hline 347.89 & 296.80 & 33.44 \\
\hline 345.49 & 295.33 & 38.71 \\
\hline 343.18 & 293.72 & 44.04 \\
\hline 341.28 & 292.49 & 49.36 \\
\hline 339.53 & 291.54 & 54.69 \\
\hline 337.56 & 289.87 & 59.46 \\
\hline 336.40 & 288.38 & 63.79 \\
\hline 335.42 & 287.35 & 67.10 \\
\hline 334.85 & 286.89 & 70.65 \\
\hline 332.07 & 285.56 & 75.54 \\
\hline 329.71 & 284.11 & 80.48 \\
\hline 327.46 & 282.63 & 86.99 \\
\hline 324.43 & 280.31 & 94.48 \\
\hline 322.10 & 278.04 & 100.45 \\
\hline 320.24 & 276.27 & 105.44 \\
\hline 318.14 & 274.25 & 111.29 \\
\hline 316.06 & 271.36 & 116.59 \\
\hline 314.69 & 269.70 & 120.64 \\
\hline 313.11 & 268.75 & 124.82 \\
\hline 310.86 & 266.05 & 127.89 \\
\hline 309.76 & 264.69 & 130.93 \\
\hline 307.88 & 263.19 & 134.82 \\
\hline 305.95 & 262.18 & 138.38 \\
\hline 304.41 & 261.52 & 142.60 \\
\hline 302.61 & 261.17 & 147.03 \\
\hline 300.71 & 260.17 & 150.94 \\
\hline 298.29 & 257.36 & 155.11 \\
\hline 296.12 & 255.32 & 159.37 \\
\hline 293.61 & 254.64 & 163.53 \\
\hline 292.26 & 254.33 & 167.83 \\
\hline
\end{tabular}


Table 7 (Continued)

\begin{tabular}{ccc}
\hline $\begin{array}{c}\text { Maximum } \\
\text { Elevation } \\
(\mathrm{Ft})\end{array}$ & $\begin{array}{c}\text { Minimum } \\
\text { Elevation } \\
(\mathrm{Ft})\end{array}$ & $\begin{array}{c}\text { Distance Below } \\
\text { Kentucky Dam } \\
\text { (Miles) }\end{array}$ \\
\hline $\begin{array}{c}289.40 \\
284.57\end{array}$ & 242.35 & 172.04 \\
279.49 & 247.73 & 176.48 \\
277.59 & 247.09 & 180.55 \\
274.93 & 246.62 & 184.16 \\
272.52 & 245.97 & 188.21 \\
& & 192.35
\end{tabular}


Table 8

Envelope Profile Downstream of Kentucky Dam for

Tellico PMF Dambreak

\begin{tabular}{|c|c|c|}
\hline $\begin{array}{l}\text { Maximum } \\
\text { Elevation } \\
\text { (Ft) } \\
\end{array}$ & $\begin{array}{l}\text { Minimum } \\
\text { Elevation } \\
\text { (Ft) } \\
\end{array}$ & $\begin{array}{c}\text { Distance Below } \\
\text { Kentucky Dam } \\
\text { (Miles) } \\
\end{array}$ \\
\hline 352.62 & 303.69 & 0.00 \\
\hline 352.54 & 303.30 & 3.82 \\
\hline 352.39 & 302.77 & 7.41 \\
\hline 352.24 & 302.27 & 11.00 \\
\hline 352.14 & 301.88 & 14.54 \\
\hline 351.97 & 301.39 & 18.03 \\
\hline 351.60 & 299.84 & 19.88 \\
\hline 351.15 & 299.81 & 22.58 \\
\hline 350.59 & 299.41 & 23.53 \\
\hline 350.22 & 299.08 & 24.28 \\
\hline 349.84 & 298.76 & 24.98 \\
\hline 349.50 & 298.49 & 28.06 \\
\hline 346.96 & 296.80 & 33.44 \\
\hline 344.79 & 295.64 & 38.71 \\
\hline 342.71 & 294.31 & 44.04 \\
\hline 340.99 & 293.16 & 49.36 \\
\hline 339.40 & 292.22 & 54.69 \\
\hline 337.60 & 290.57 & 59.46 \\
\hline 336.53 & 289.08 & 63.79 \\
\hline 335.62 & 288.01 & 67.10 \\
\hline 335.06 & 287.54 & 70.65 \\
\hline 332.32 & 286.19 & 75.54 \\
\hline 329.98 & 284.71 & 80.48 \\
\hline 327.75 & 283.19 & 86.99 \\
\hline 324.73 & 280.82 & 94.48 \\
\hline 322.40 & 278.42 & 100.45 \\
\hline 320.54 & 276.45 & 105.44 \\
\hline 318.45 & 274.25 & 111.29 \\
\hline 316.36 & 271.36 & 116.59 \\
\hline 314.99 & 269.70 & 120.64 \\
\hline 313.42 & 268.75 & 124.82 \\
\hline 311.17 & 266.05 & 127.89 \\
\hline 310.07 & 264.69 & 130.93 \\
\hline 308.18 & 263.19 & 134.82 \\
\hline 306.25 & 262.18 & 138.38 \\
\hline 304.71 & 261.52 & 142.60 \\
\hline 302.90 & 261.17 & 147.03 \\
\hline 300.99 & 260.17 & 150.94 \\
\hline 298.57 & 257.36 & 155.11 \\
\hline 296.39 & 255.32 & 159.37 \\
\hline 293.86 & 254.64 & 163.53 \\
\hline
\end{tabular}


Table 8 (Continued)

\begin{tabular}{ccc}
\hline $\begin{array}{c}\text { Maximum } \\
\begin{array}{c}\text { Elevation } \\
\text { (Ft) }\end{array}\end{array}$ & $\begin{array}{c}\text { Minimum } \\
\text { Elevation } \\
\text { (FT) }\end{array}$ & $\begin{array}{c}\text { Distance Below } \\
\text { Kentucky Dam } \\
\text { (Miles) }\end{array}$ \\
\cline { 1 - 2 } 292.51 & 254.33 & 167.83 \\
289.64 & 252.35 & 172.04 \\
284.80 & 249.54 & 176.48 \\
279.70 & 247.73 & 180.55 \\
277.80 & 247.09 & 184.16 \\
275.10 & 246.62 & 188.21 \\
272.68 & 245.97 & 192.35
\end{tabular}




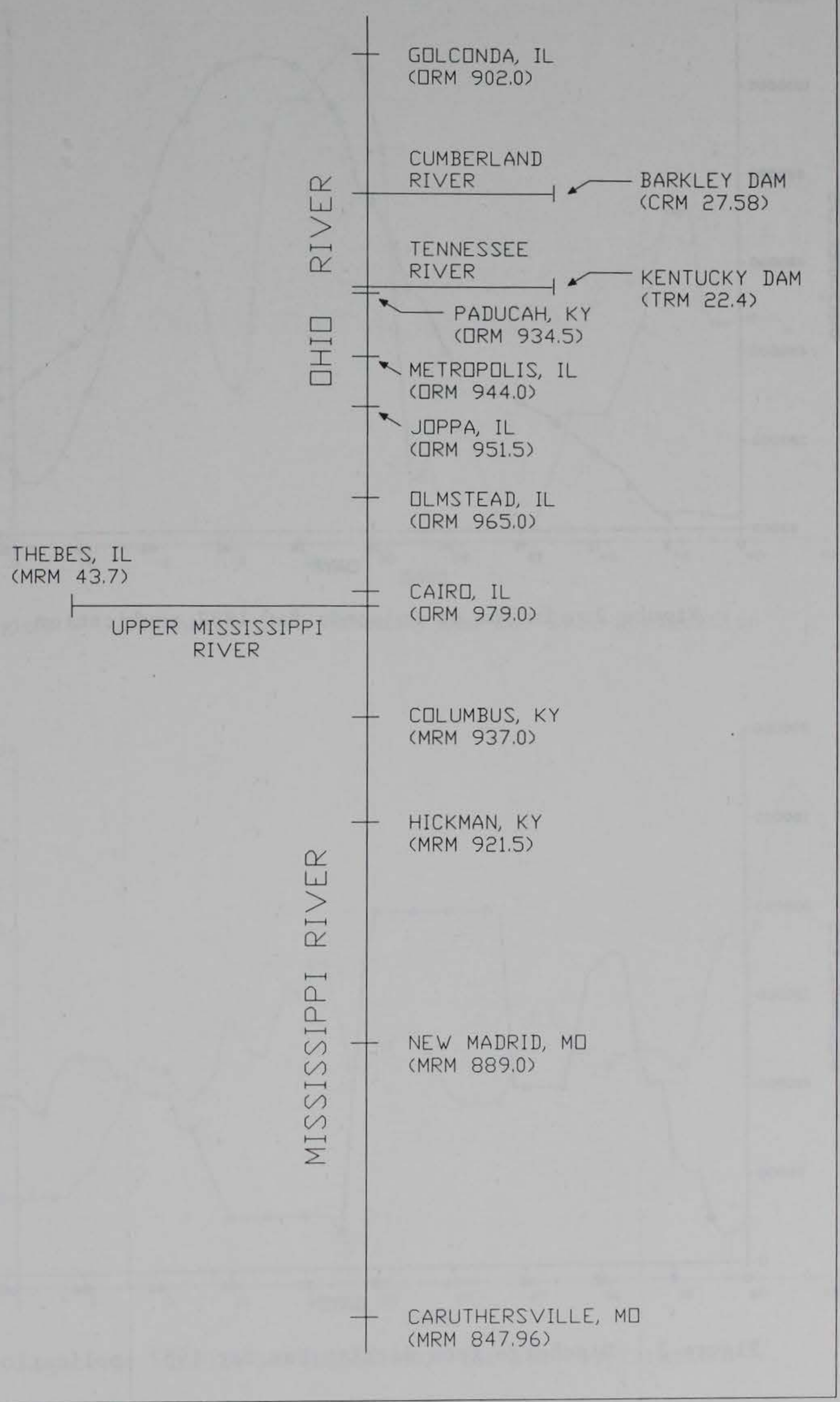




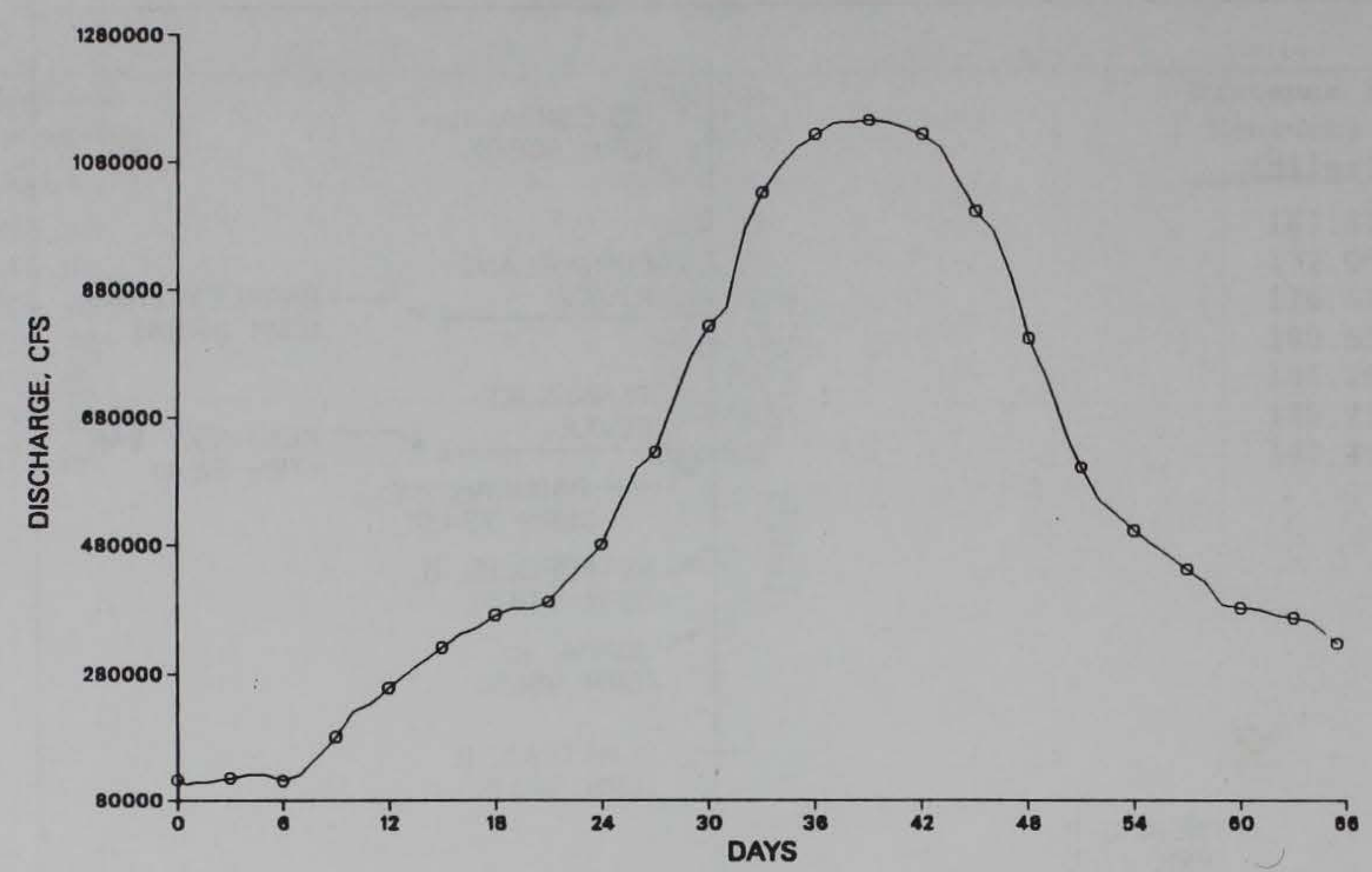

Figure 2. Inflow at Golconda for 1937 application

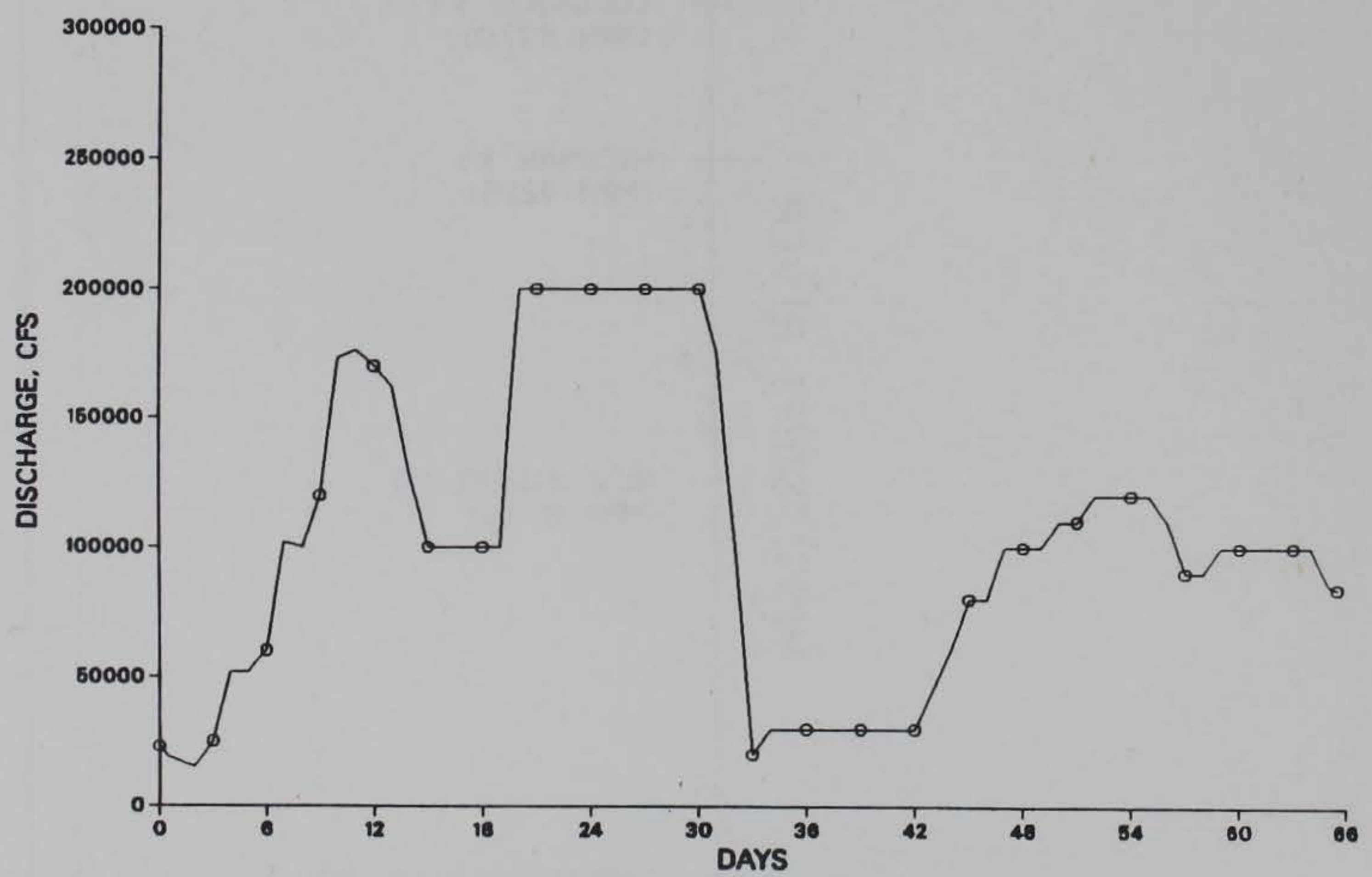

Figure 3. Discharge from Barkley Dam for 1937 application 


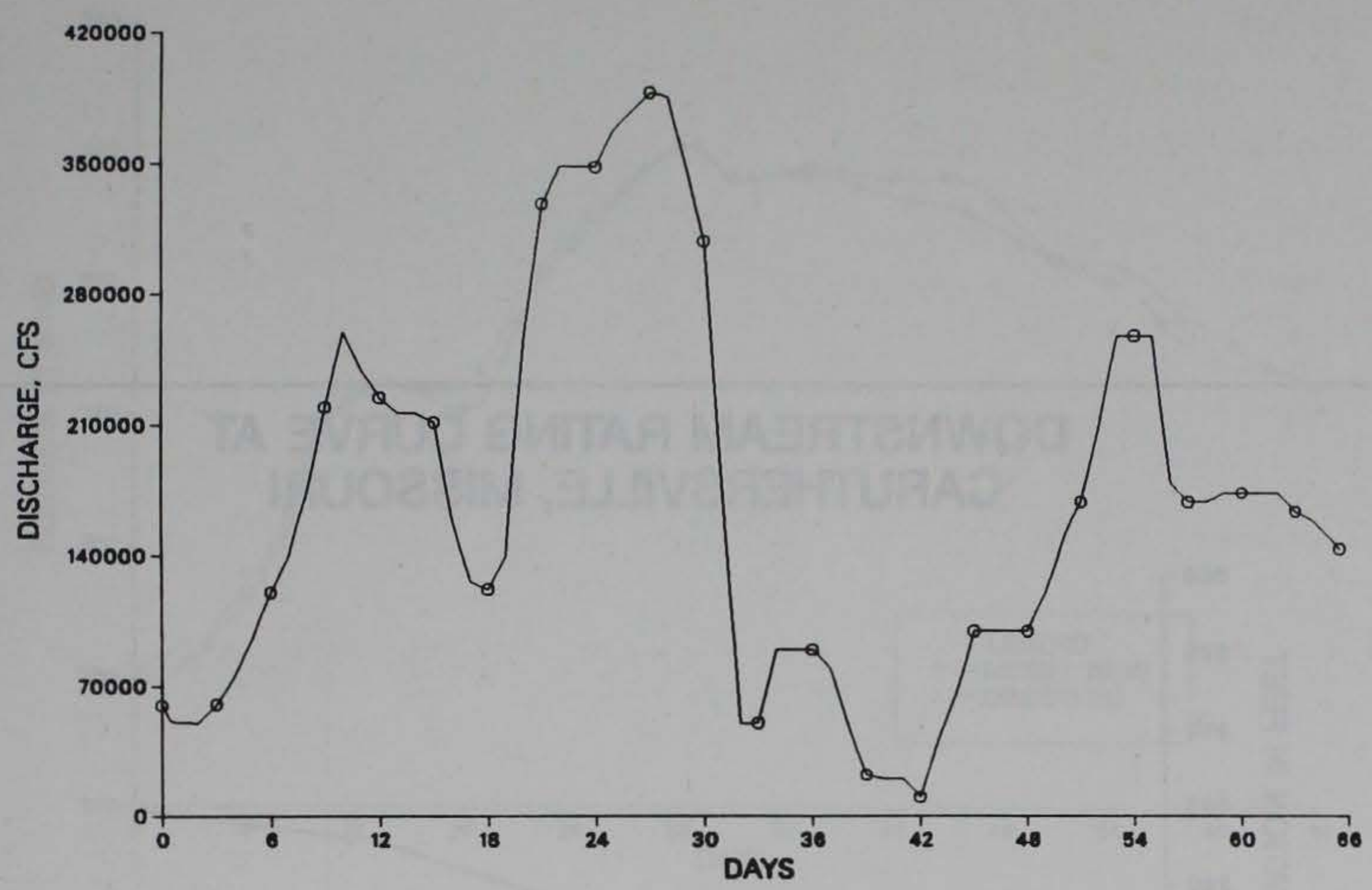

Figure 4. Discharge from Kentucky Dam for 1937 application

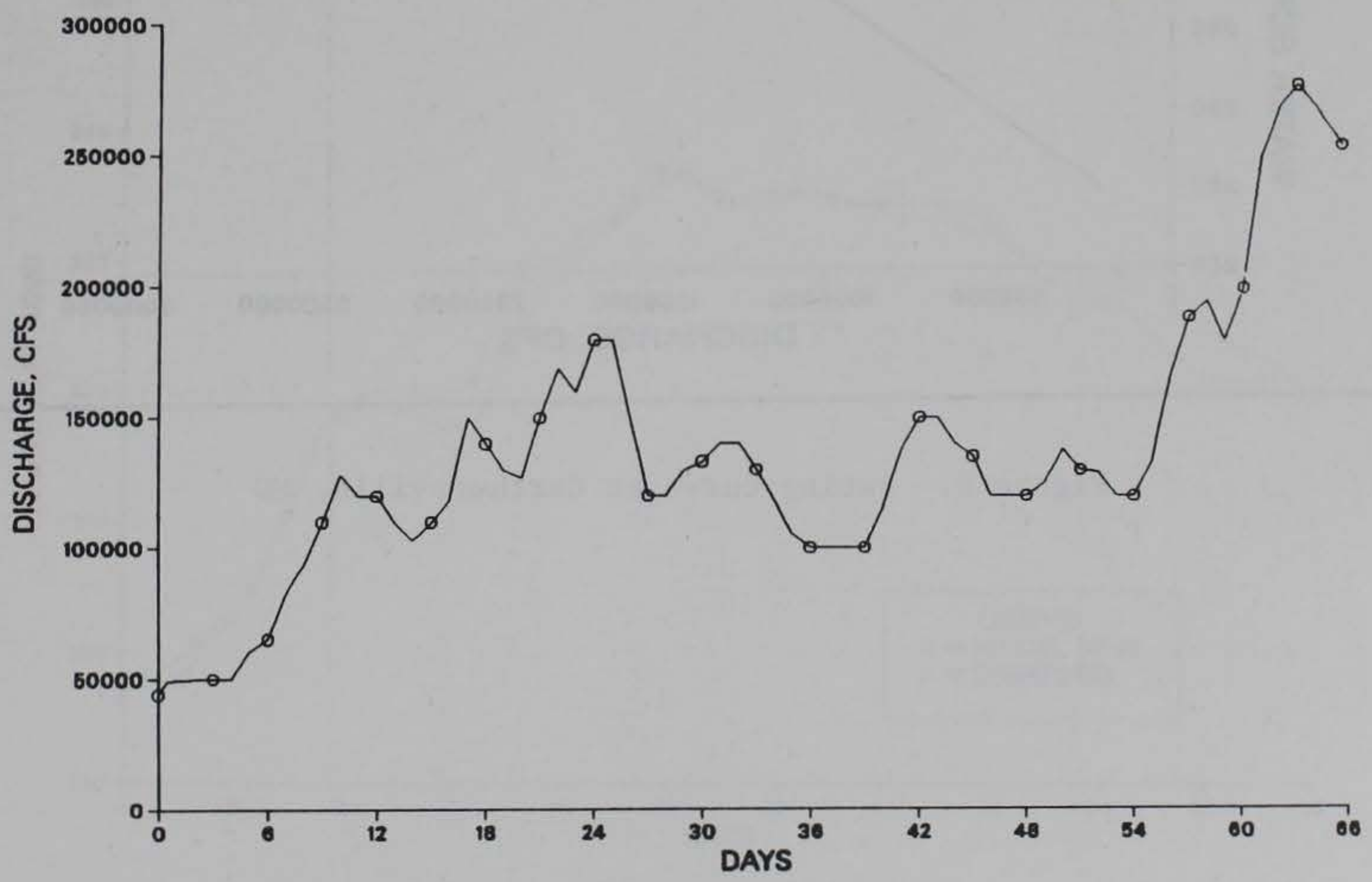

Figure 5. Inflow at Thebes, MO, for 1937 application 


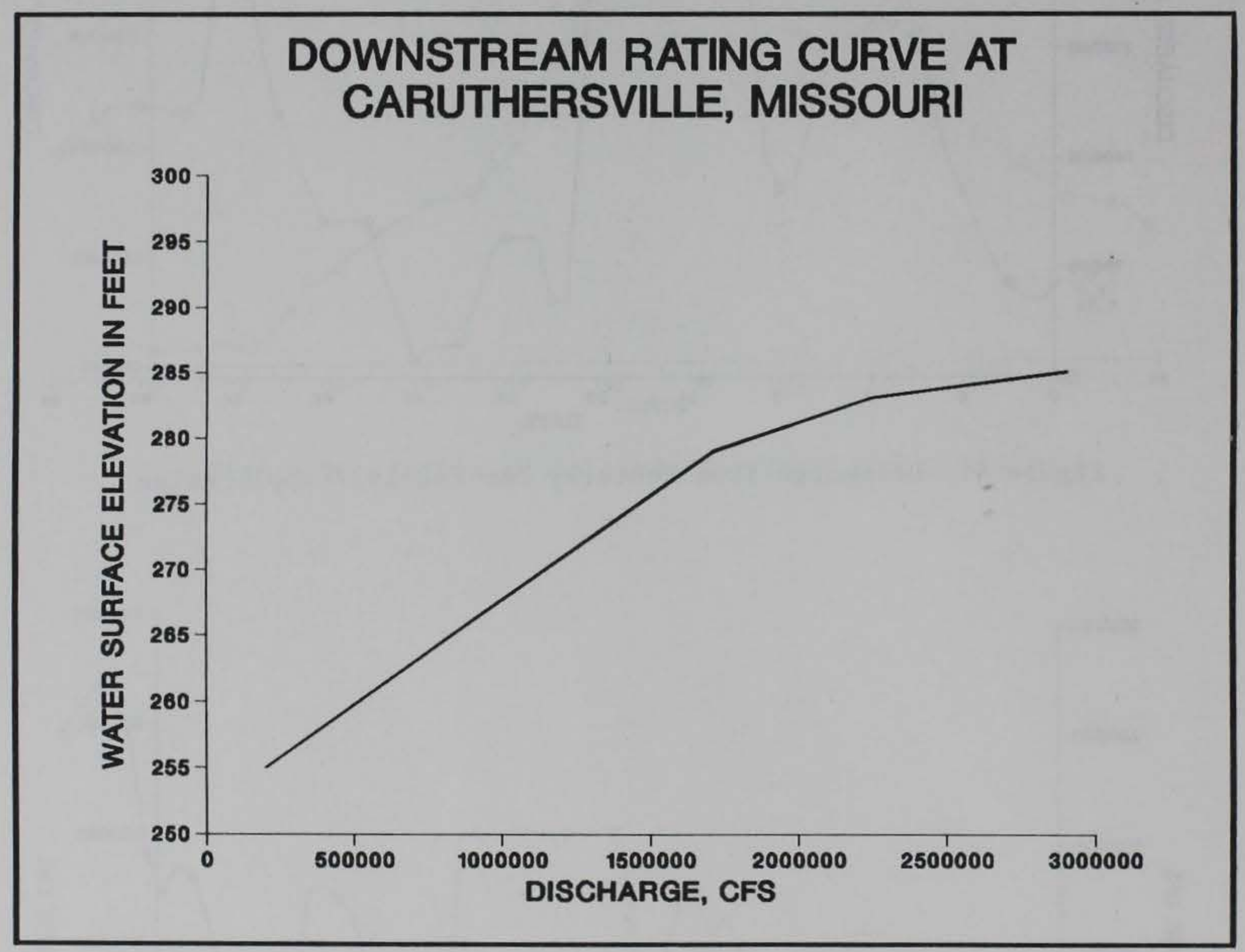

Figure 6. Rating curve at Carthersville, MO 


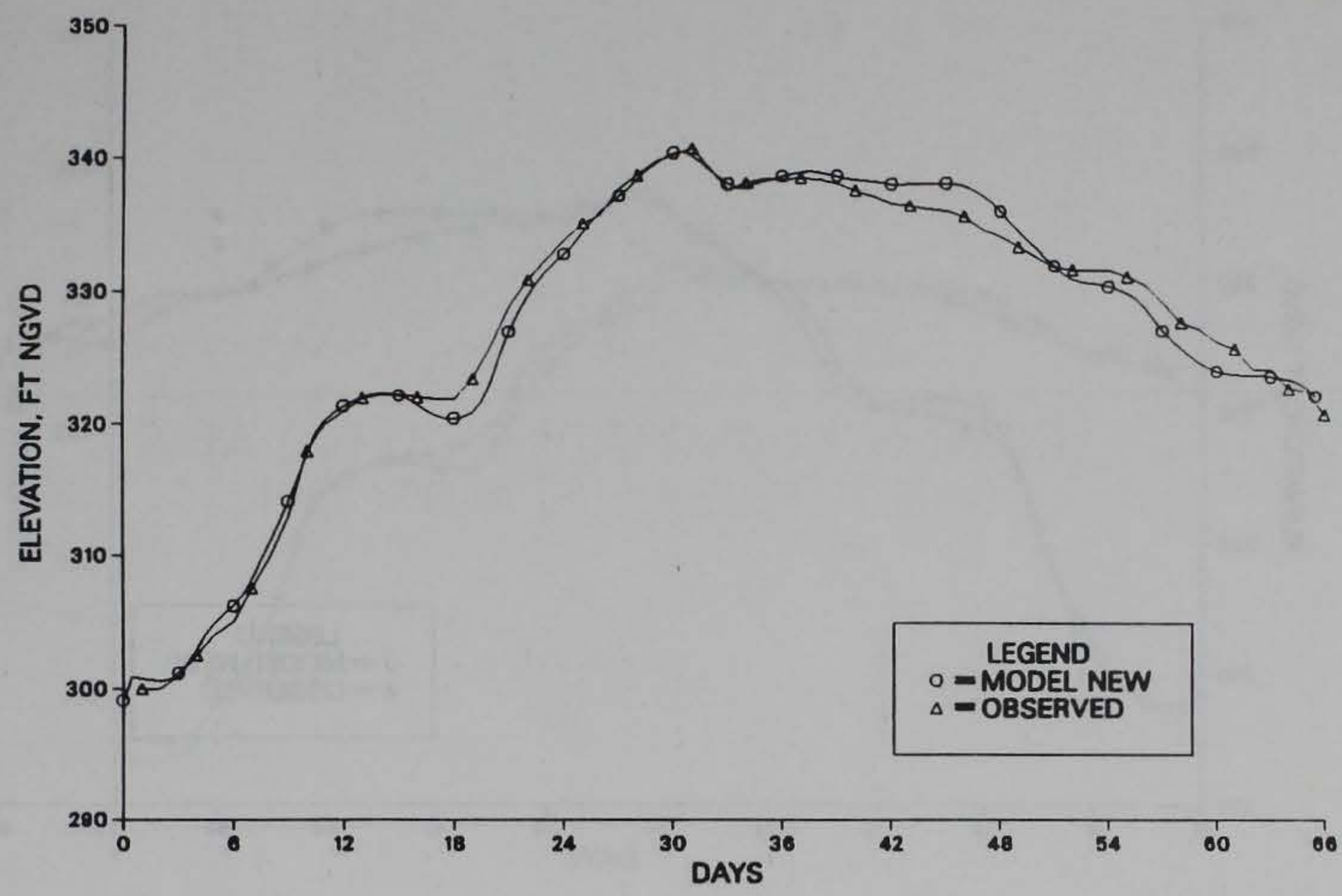

Figure 7. Comparison of 1937 computer and Mississippi Basin model elevations at Paducah, KY

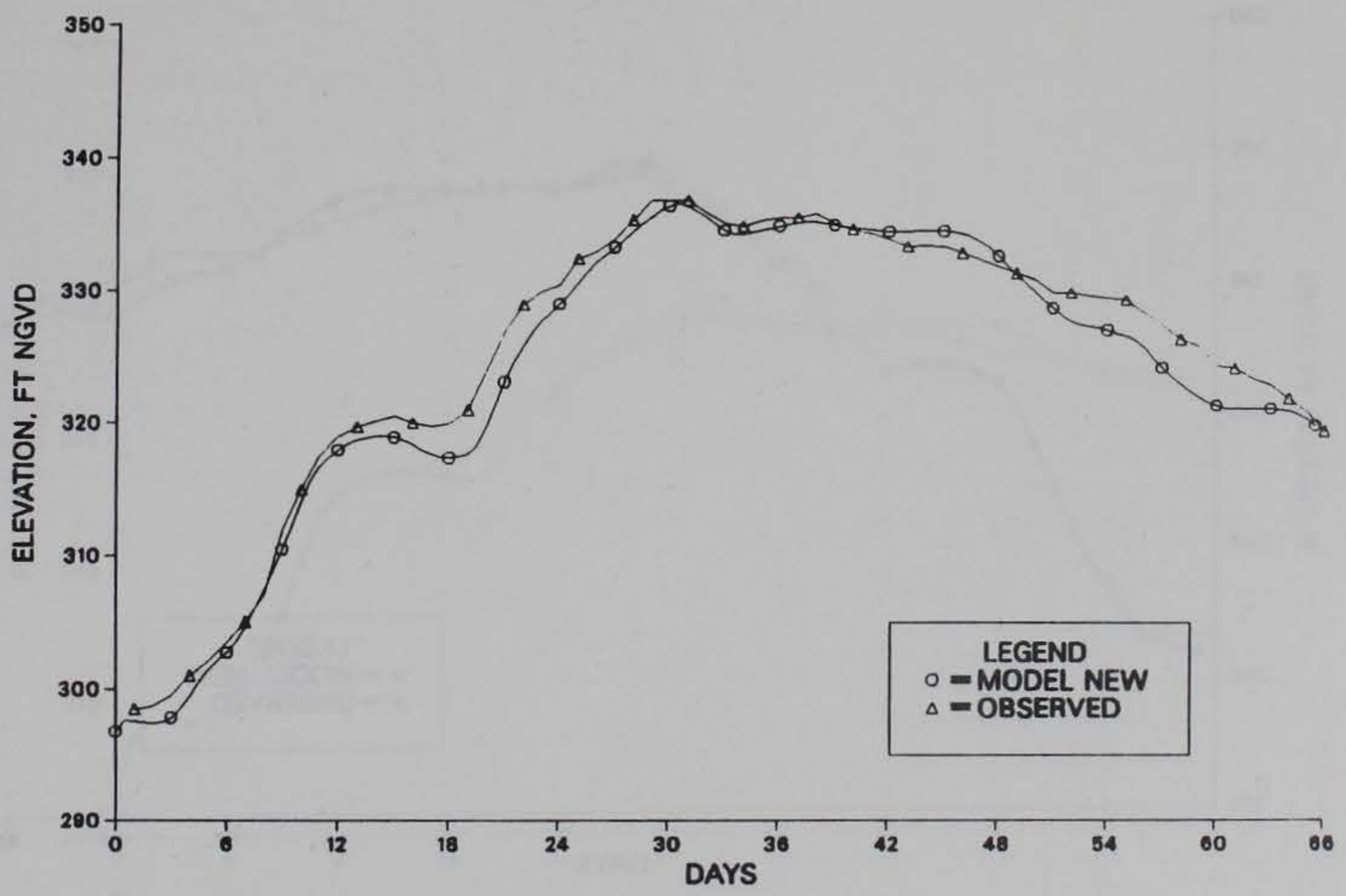

Figure 8. Comparison of 1937 computed and Mississippi Basin model elevations at Metropolis, IL 


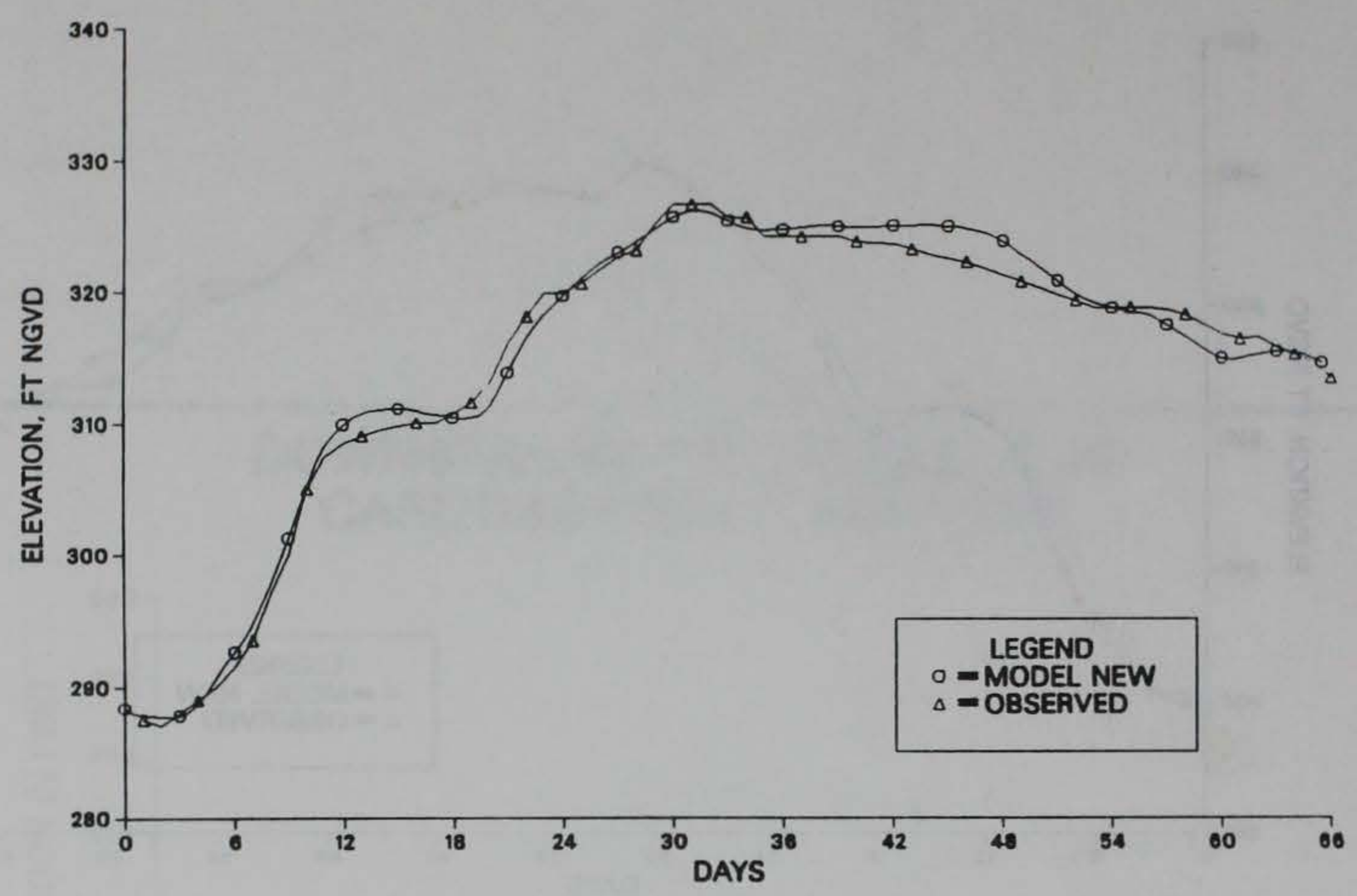

Figure 9. Comparison of 1937 computer and Mississippi Basin model elevations at Cairo, IL

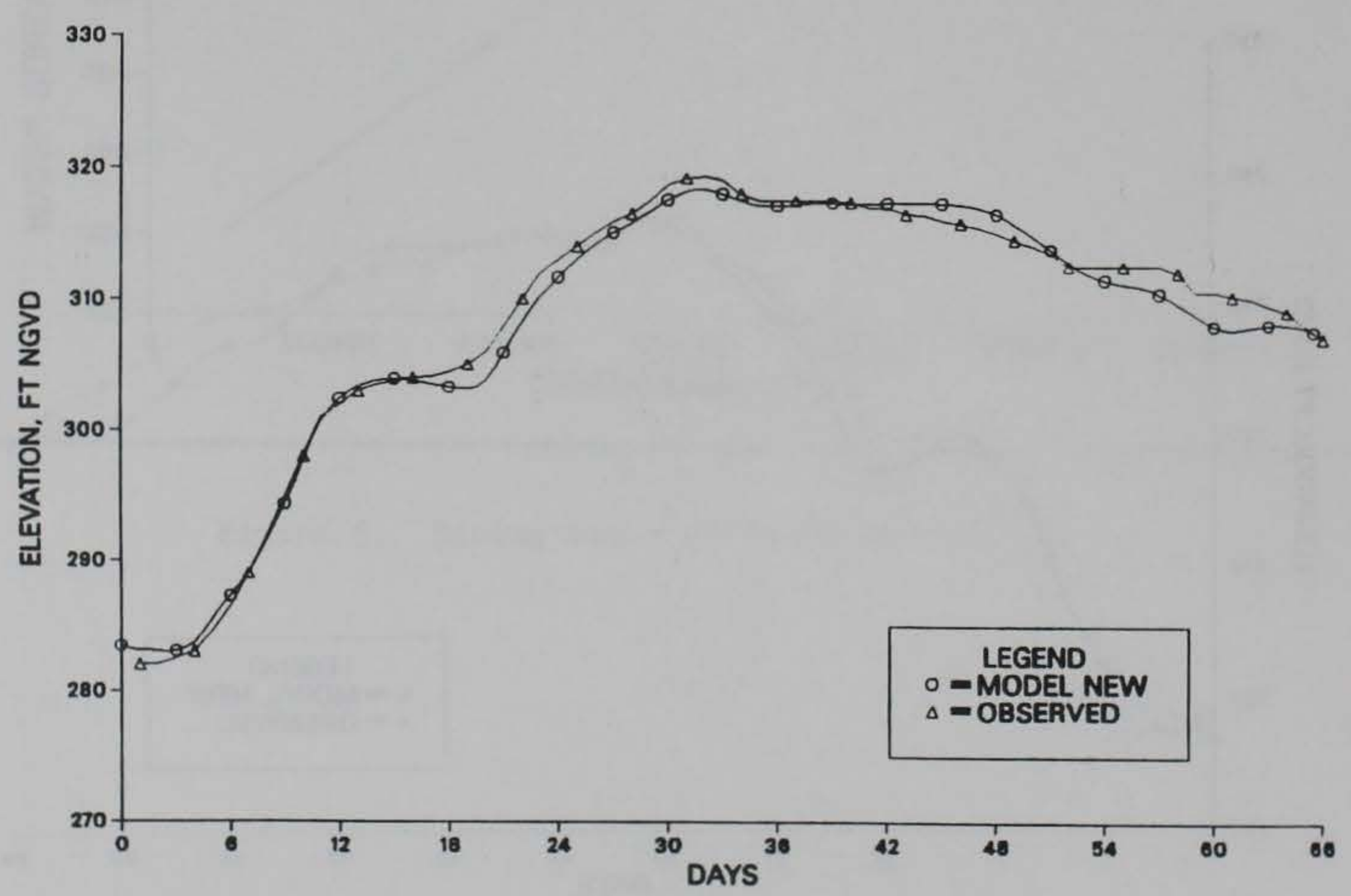

Figure 10. Comparison of 1937 computed and Mississippi Basin model elevations at Columbus, KY 


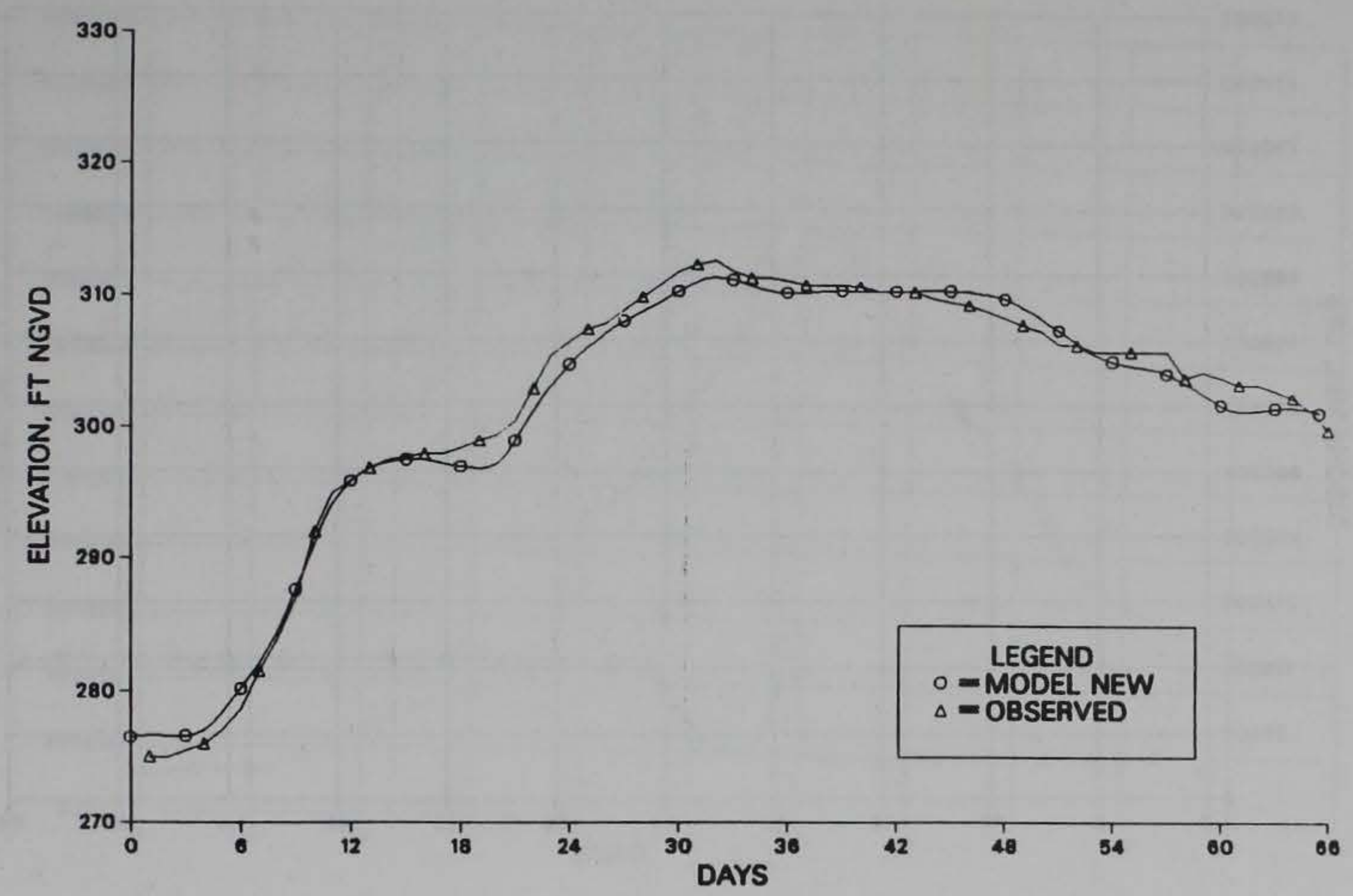

Figure 11. Comparison of 1937 computer and Mississippi Basin model elevations at Hickman, KY

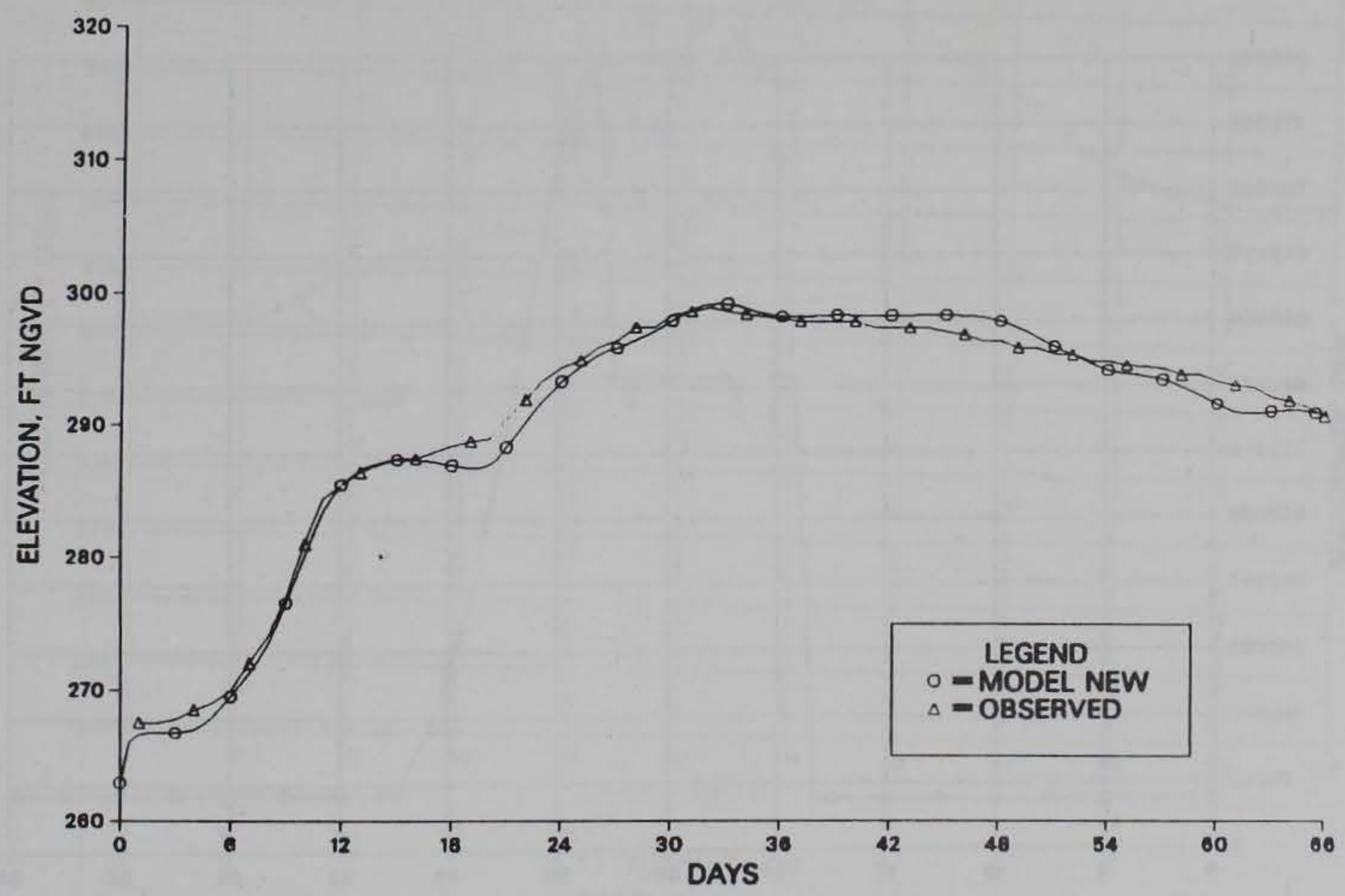

Figure 12. Comparison of 1937 computed and Mississippi Basin model elevations at New Madrid, MO 


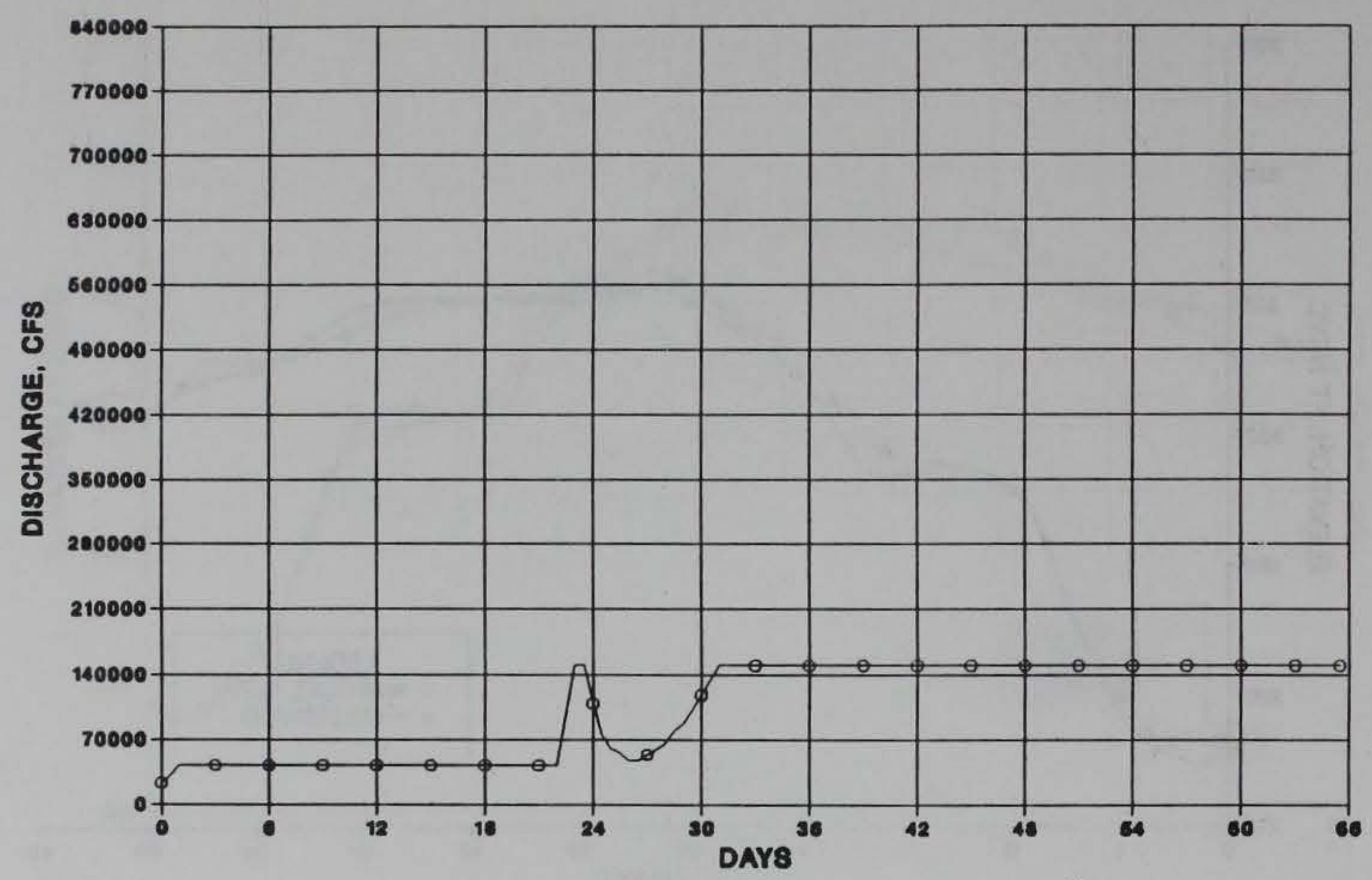

Figure 13. Discharge from Barkley Lake for Wilson PMF dam break

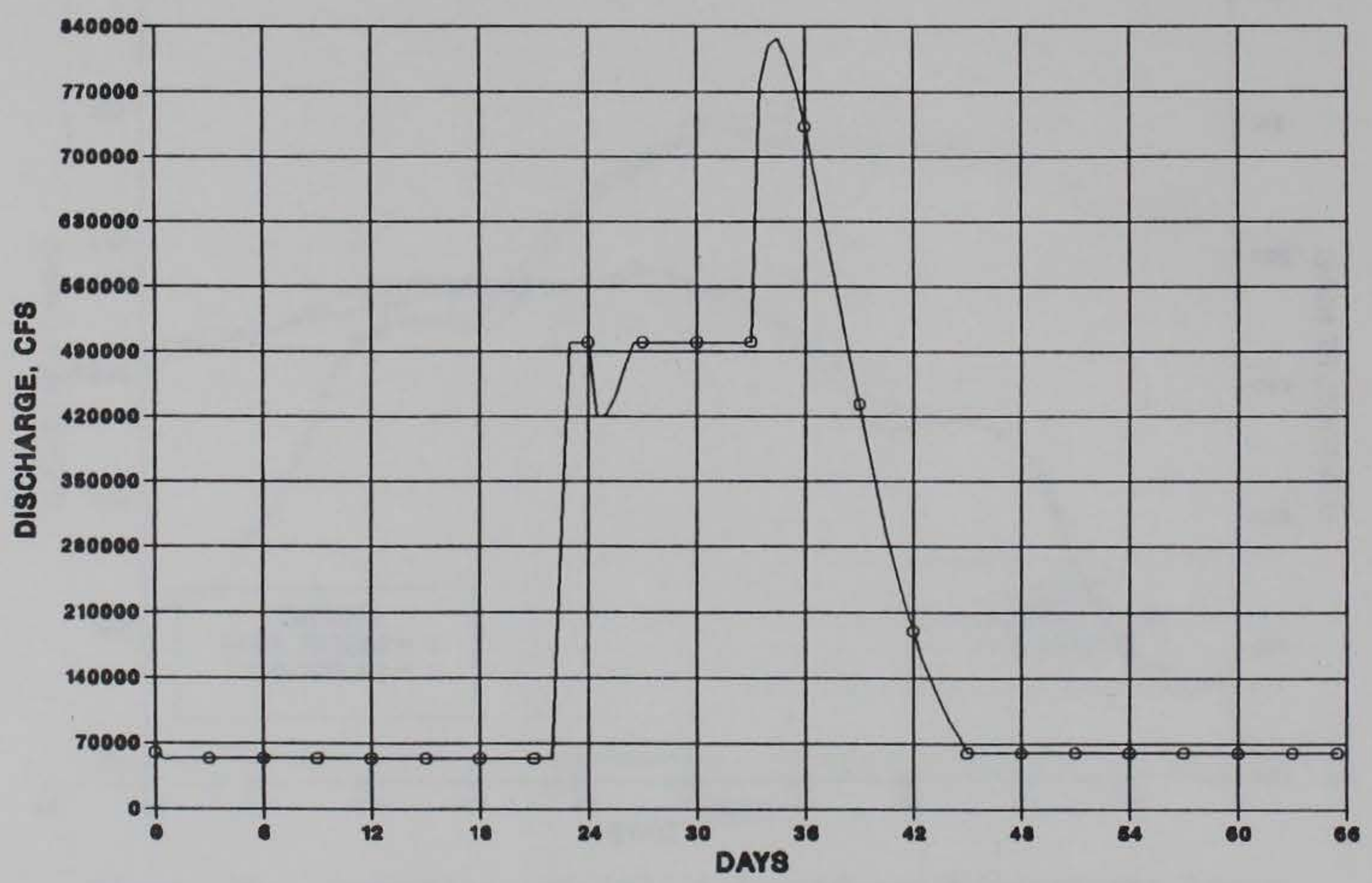

Figure 14. Discharge from Kentucky Lake for Wilson PMF dam break 


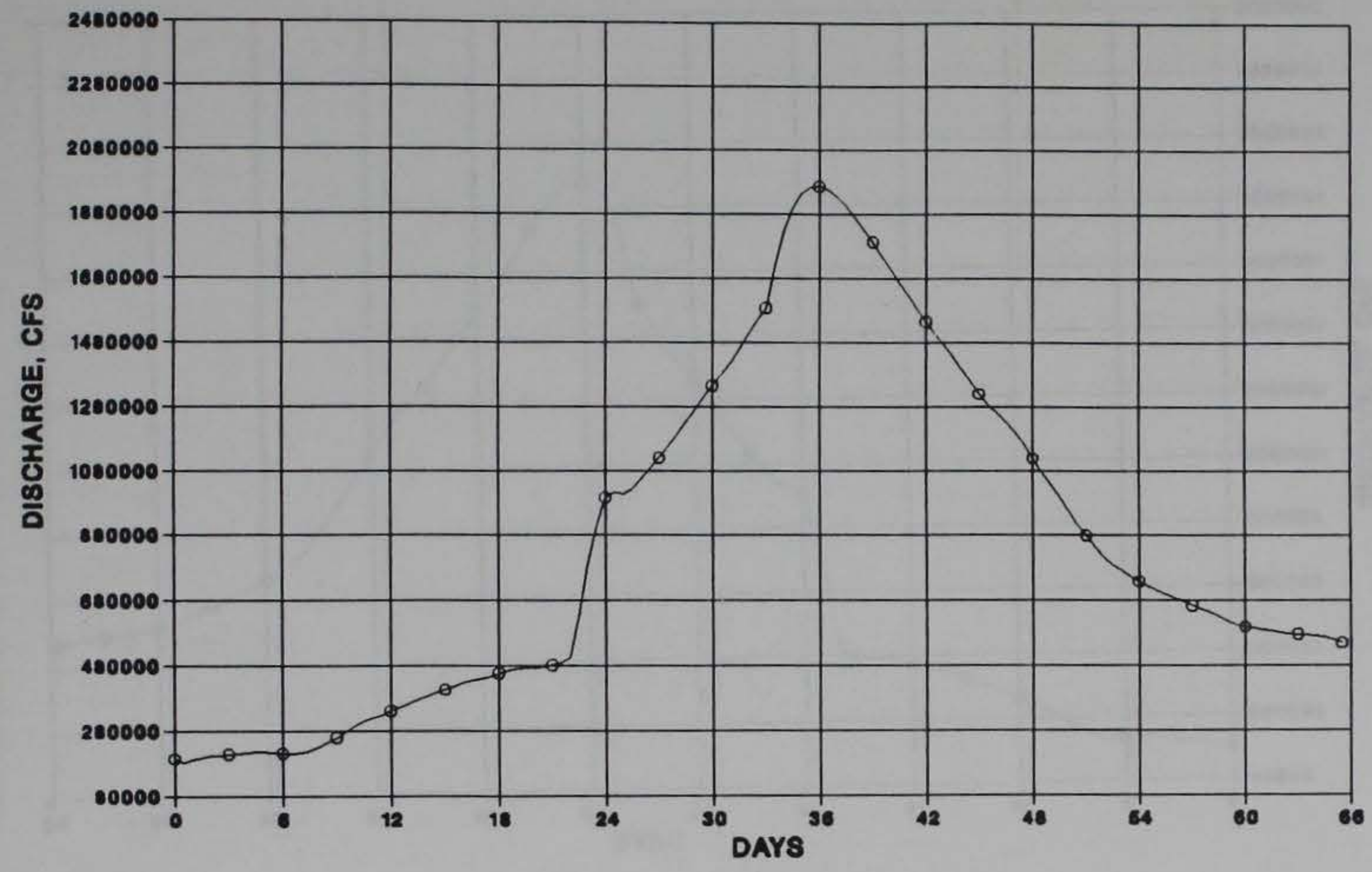

a. Discharge

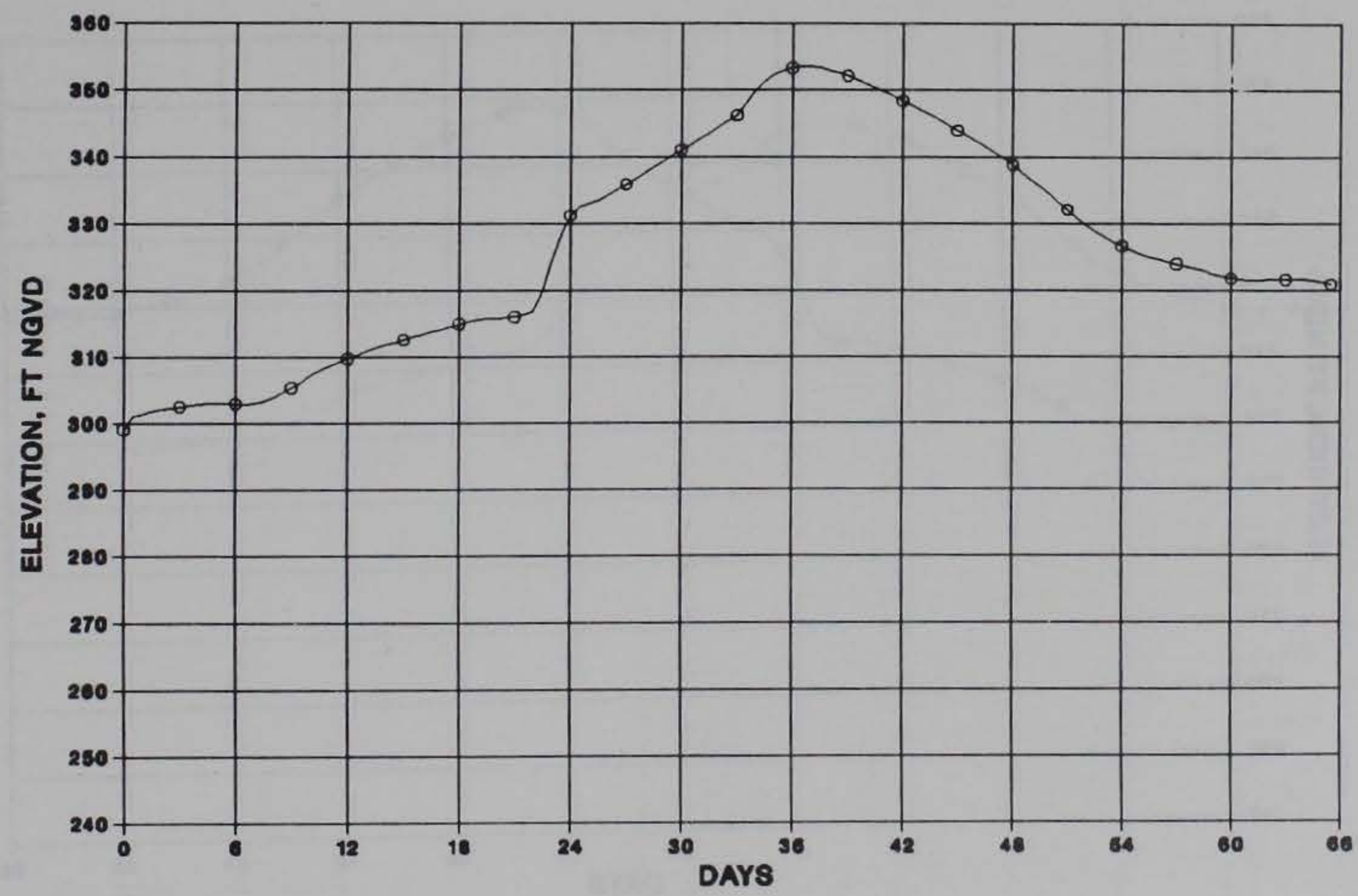

b. Elevation

Figure 15. Results at Paducah, KY, for Wilson PMF dam break 


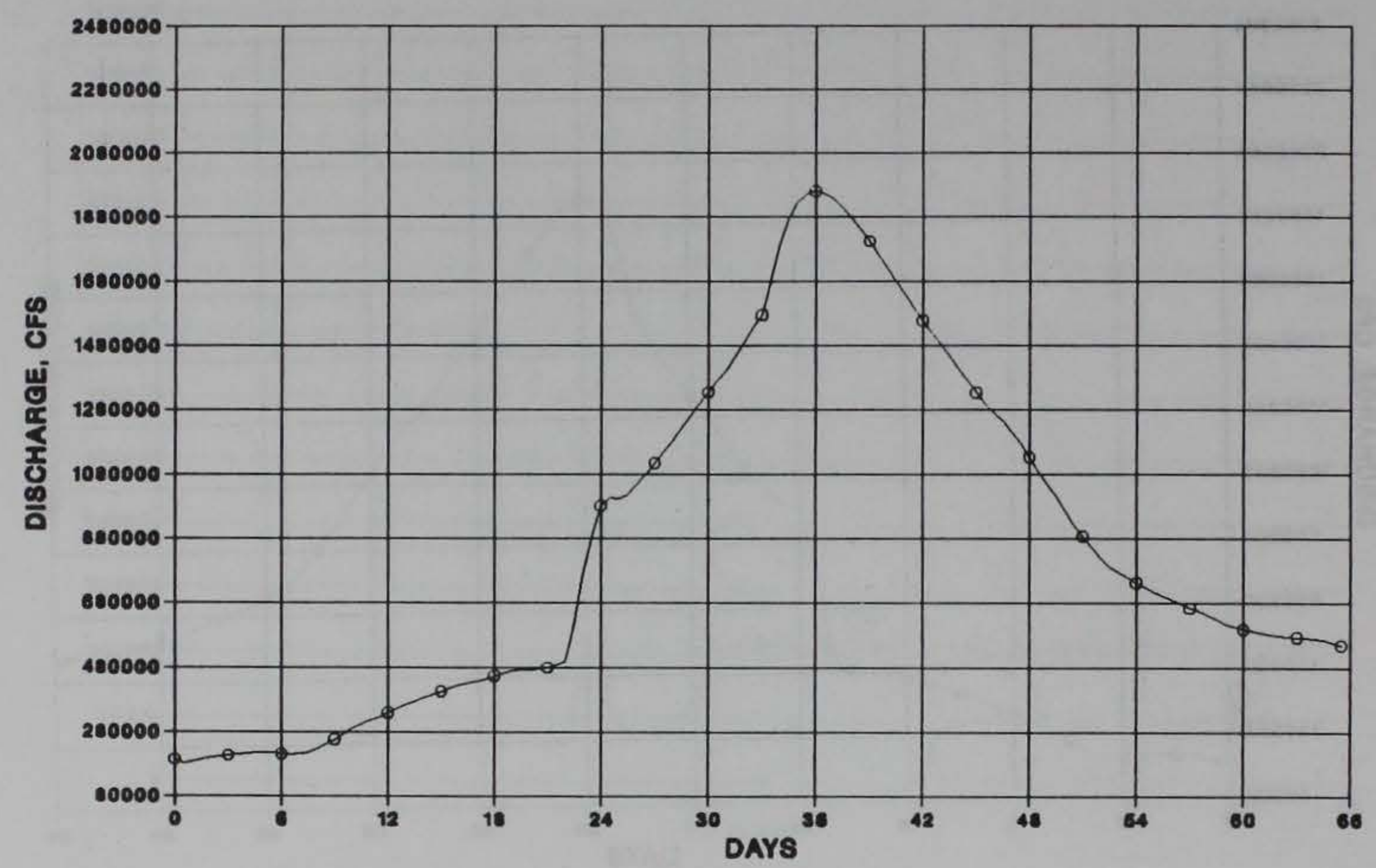

a. Discharge

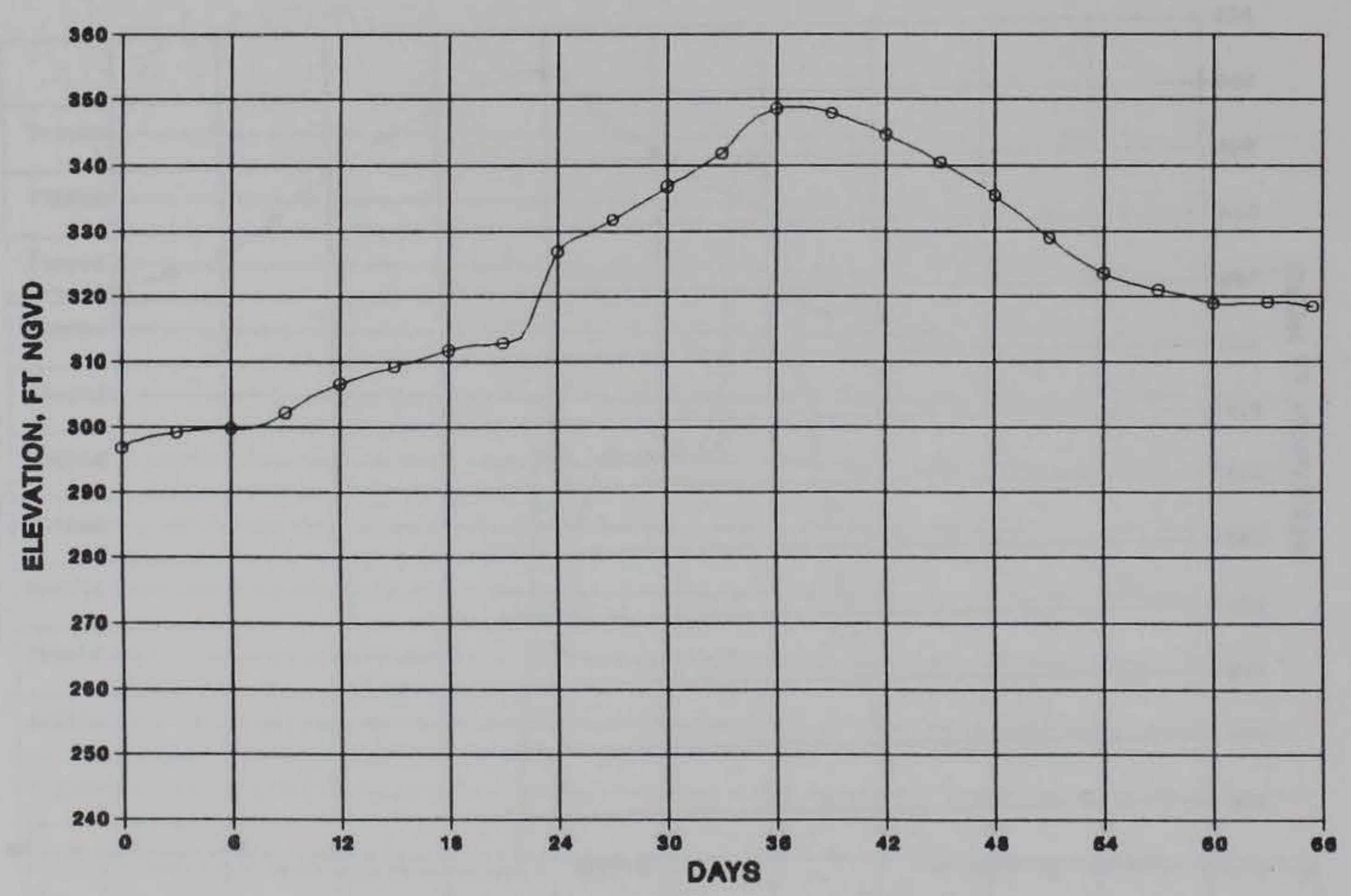

b. Elevation

Figure 16. Results at Metropolis, IL, for Wilson PMF dam break 


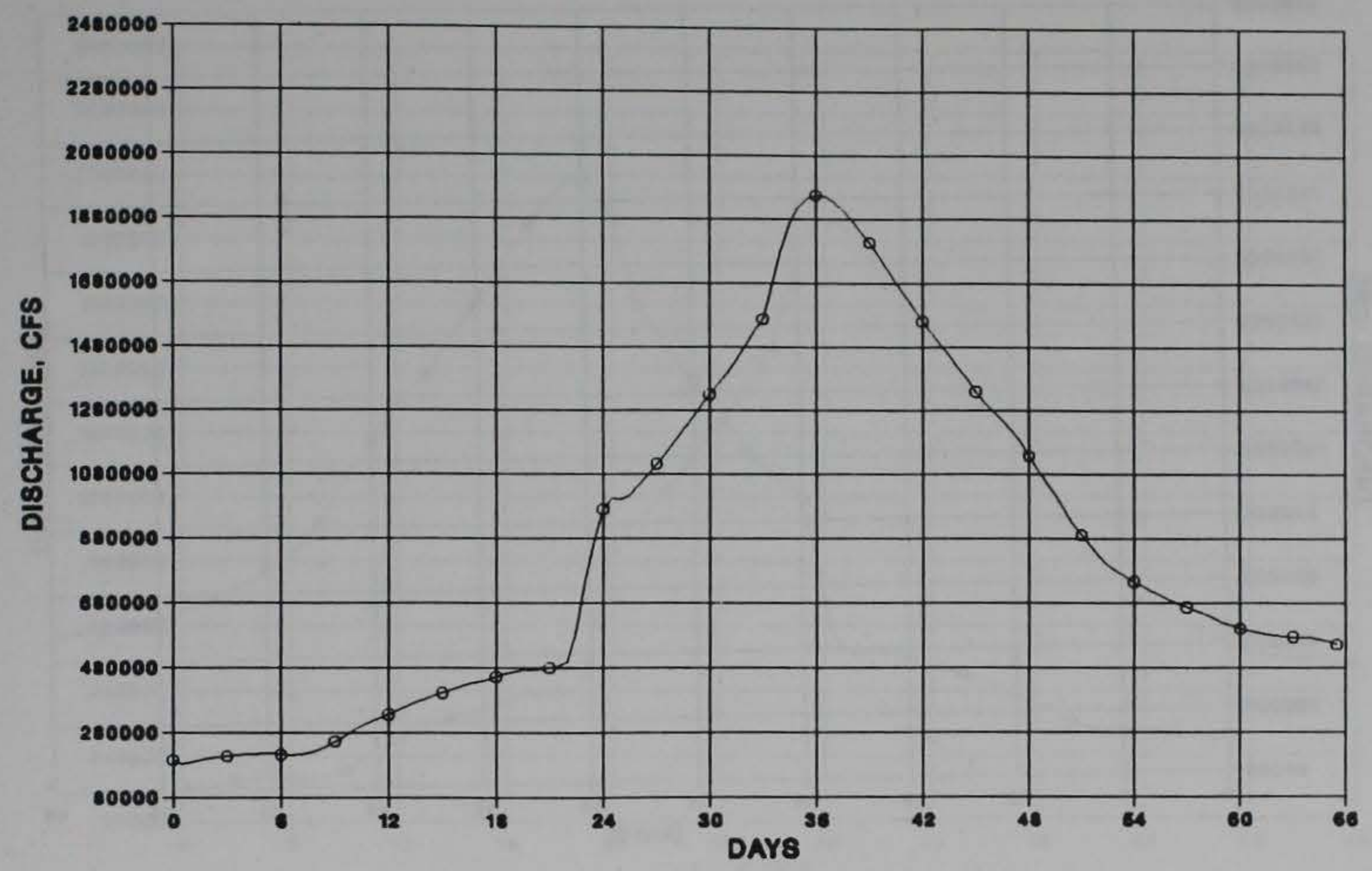

a. Discharge

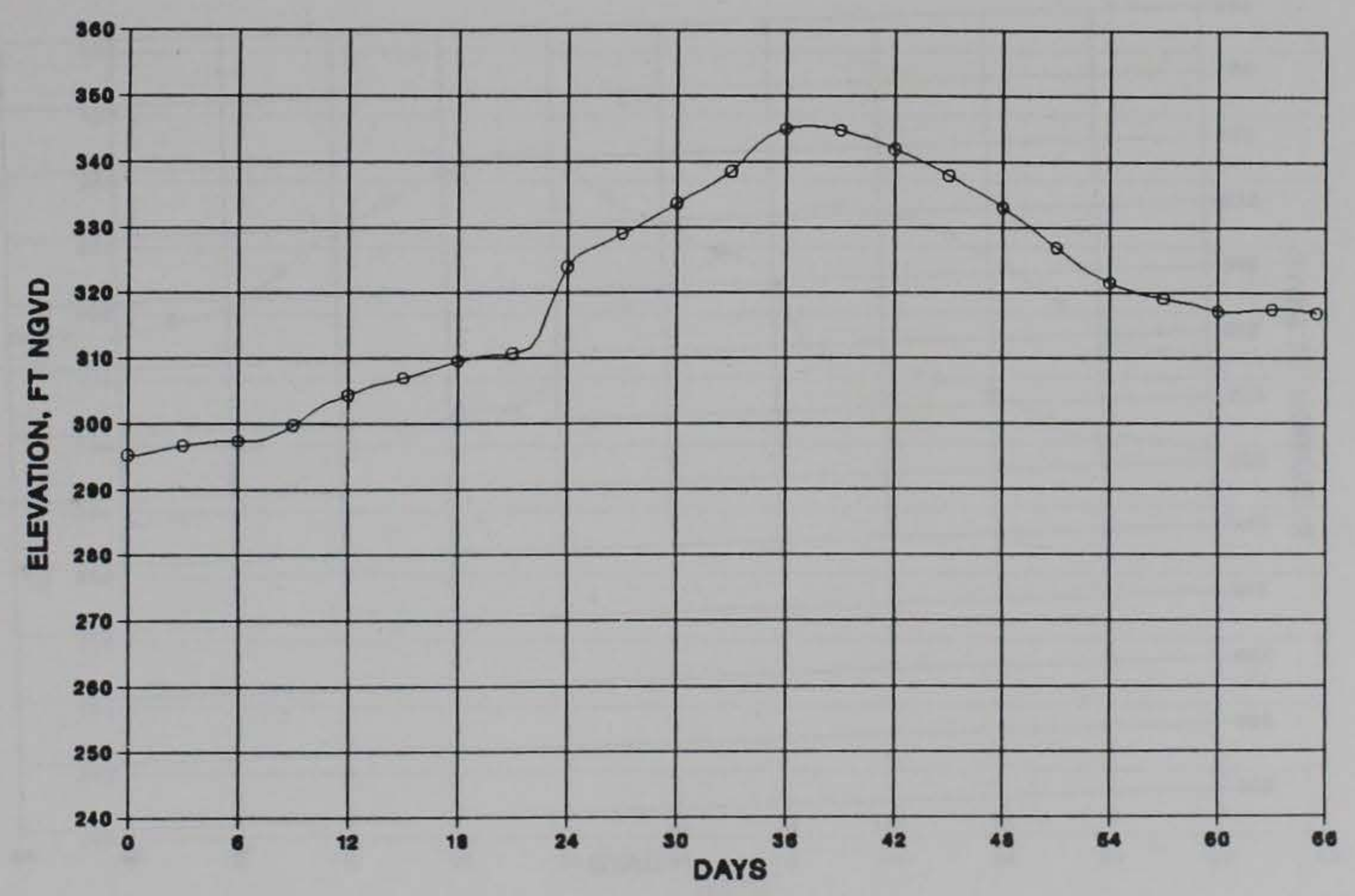

b. Elevation

Figure 17. Results at Joppa, IL, for Wilson PMF dam break 


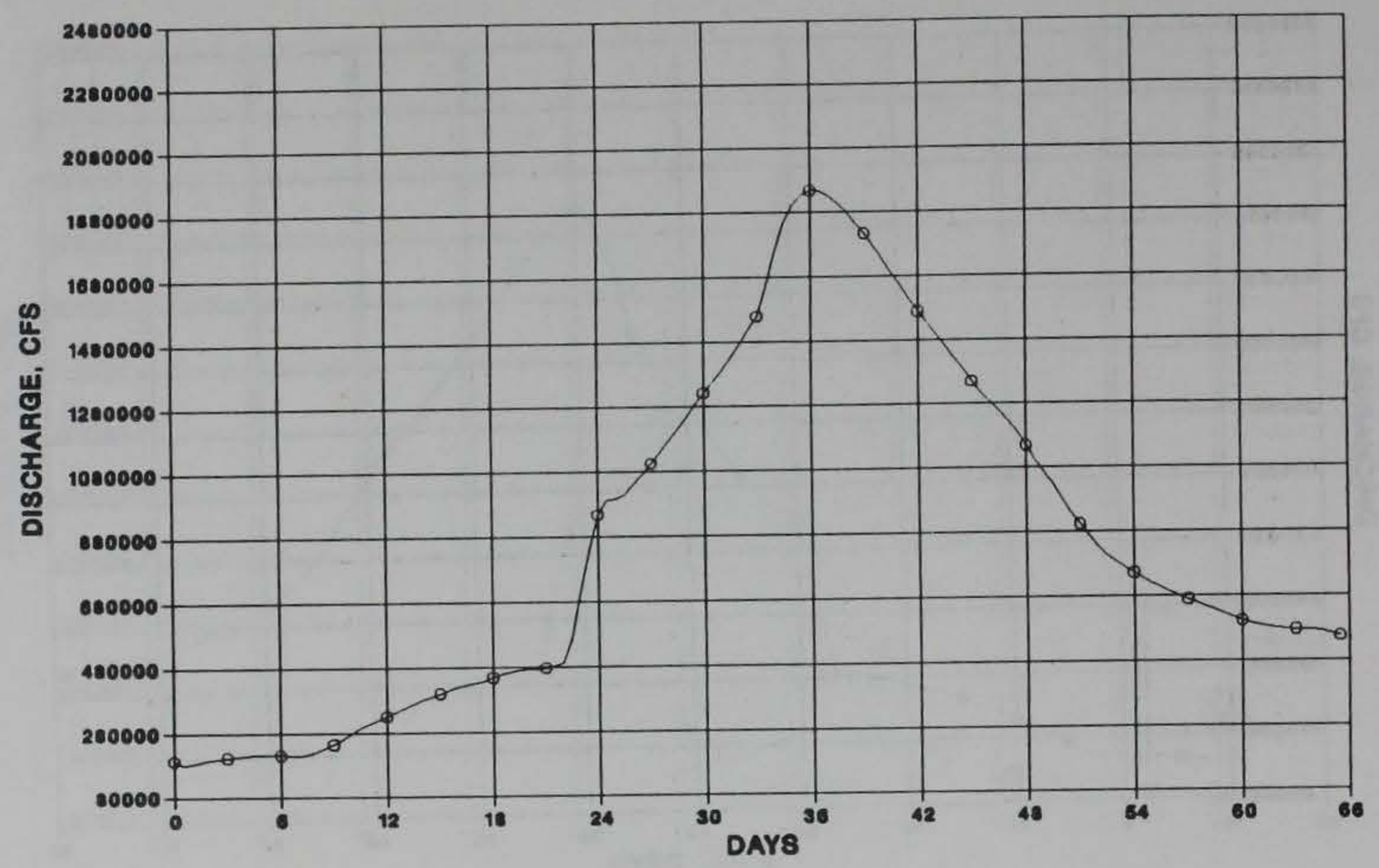

a. Discharge

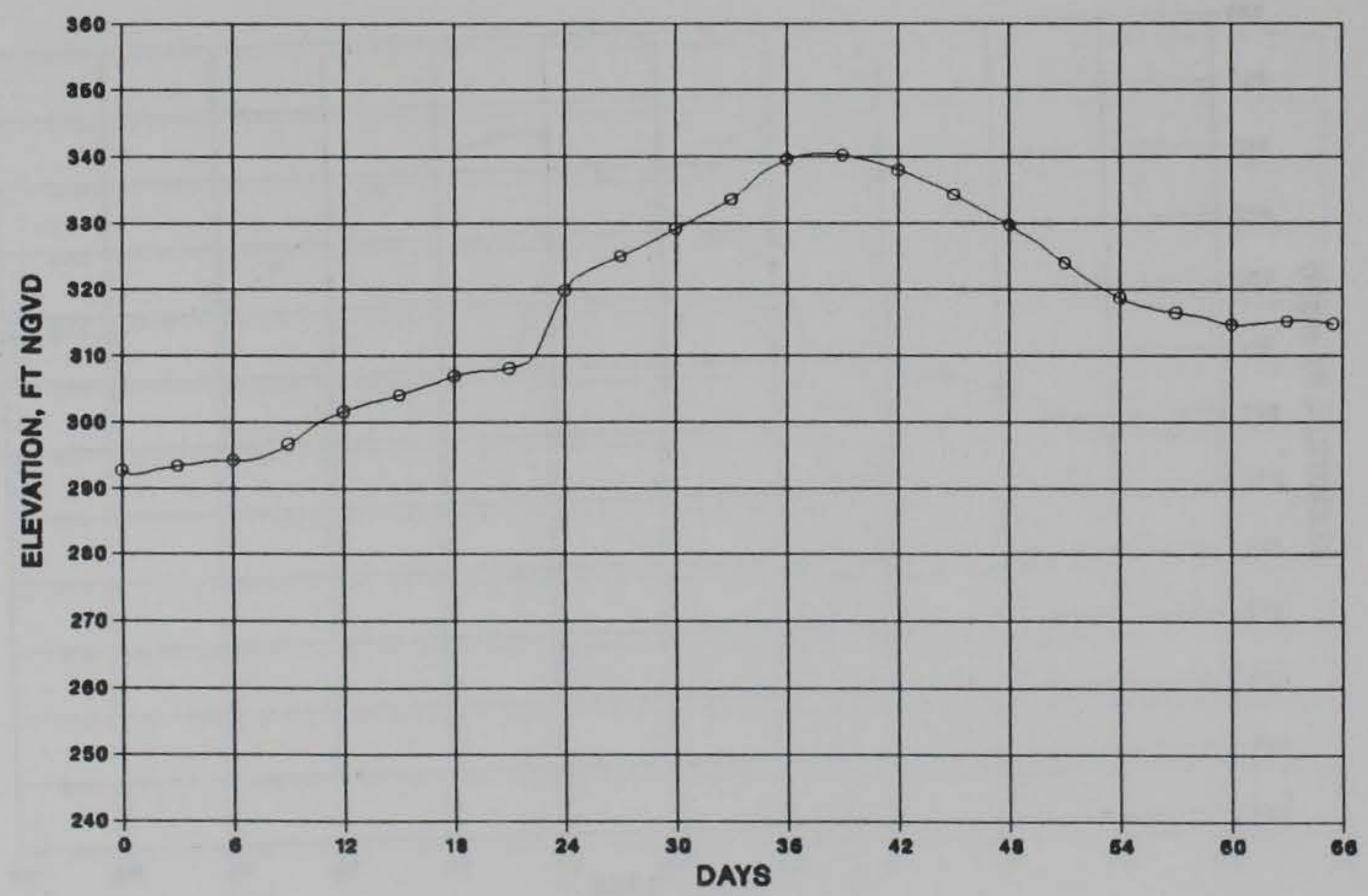

b. Elevation

Figure 18. Results at Olmsted, IL, for Wilson PMF dam break 


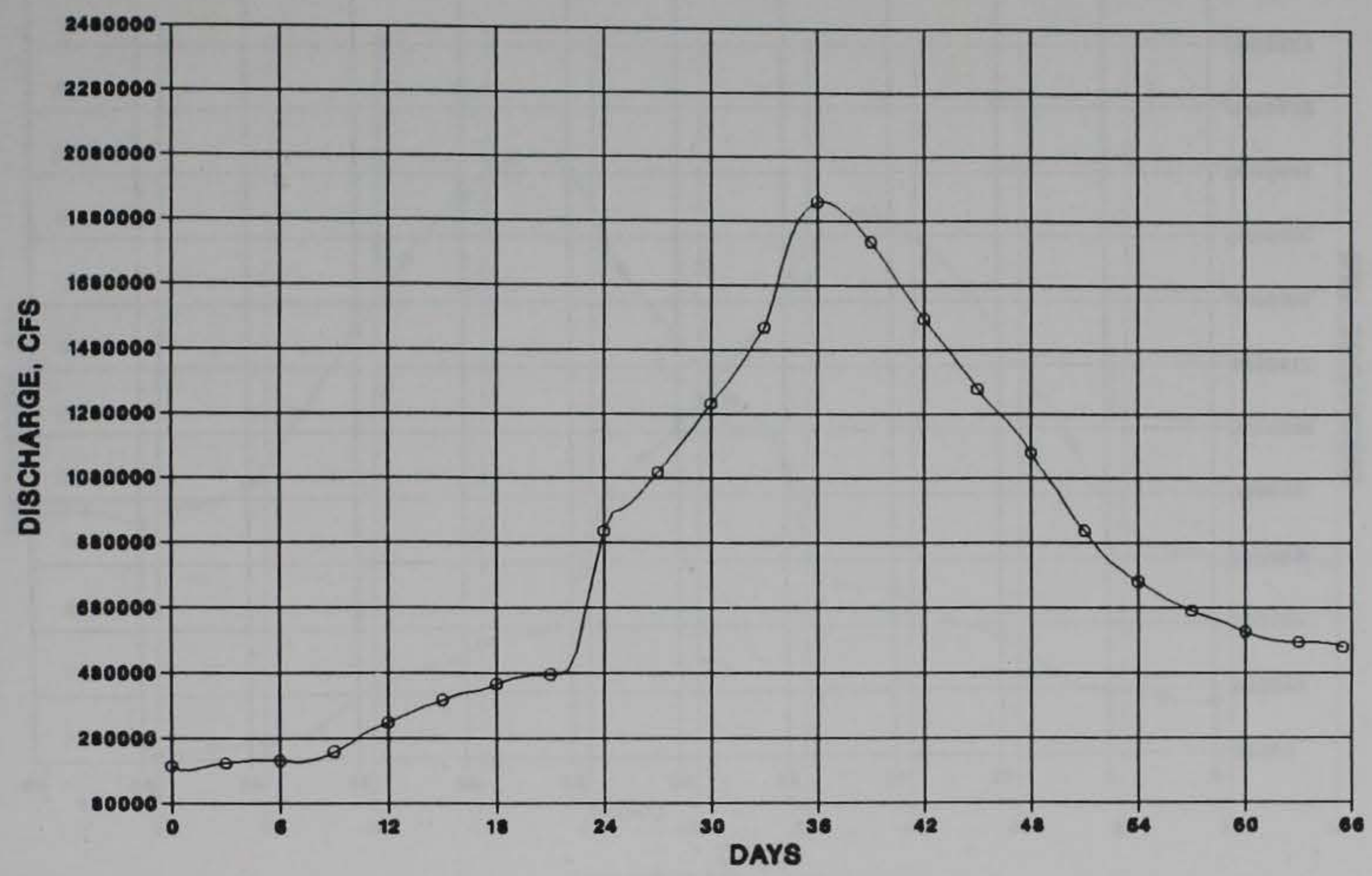

a. Discharge

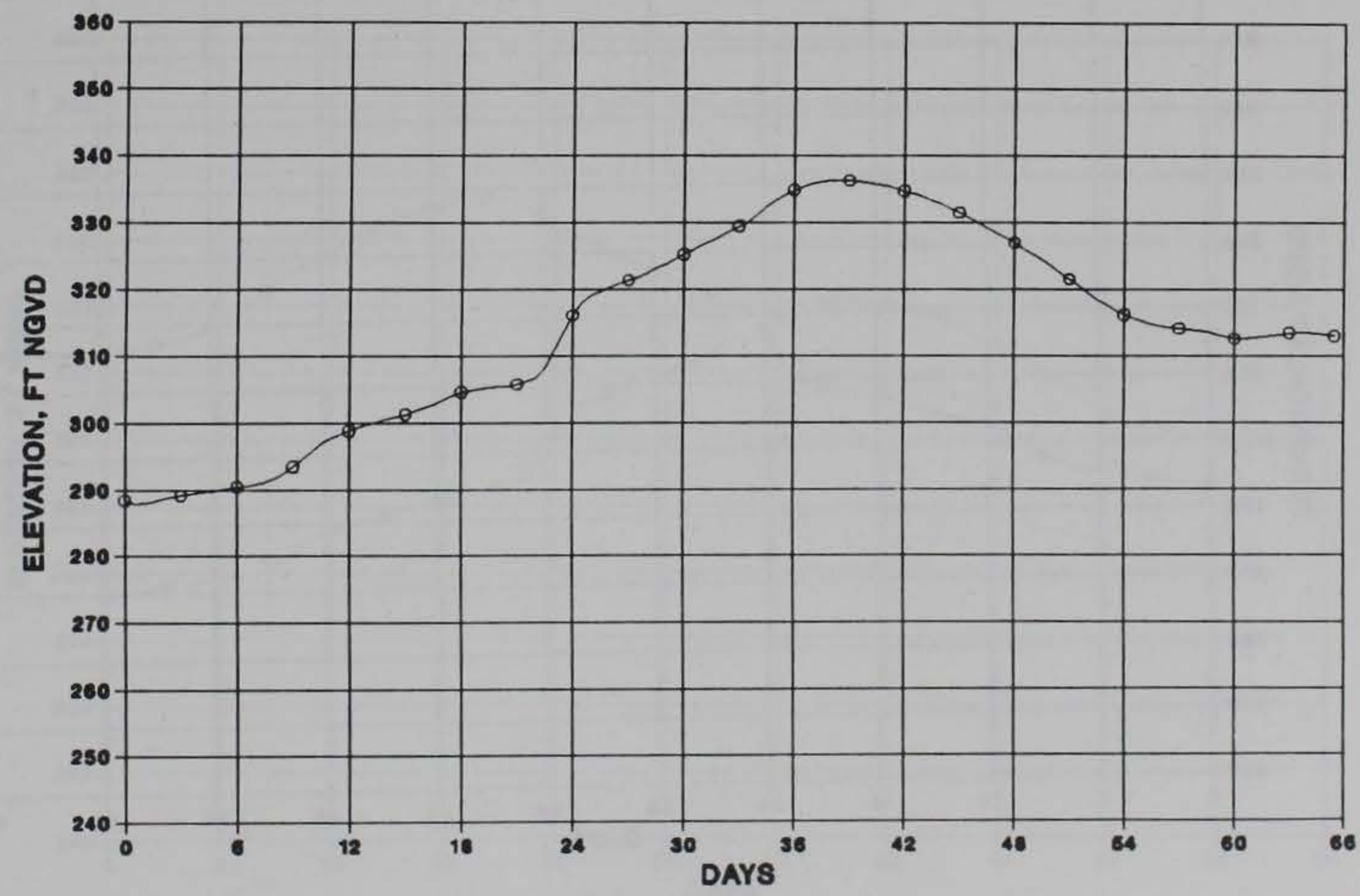

b. Elevation

Figure 19. Results at Cairo, IL, for Wilson PMF dam break 


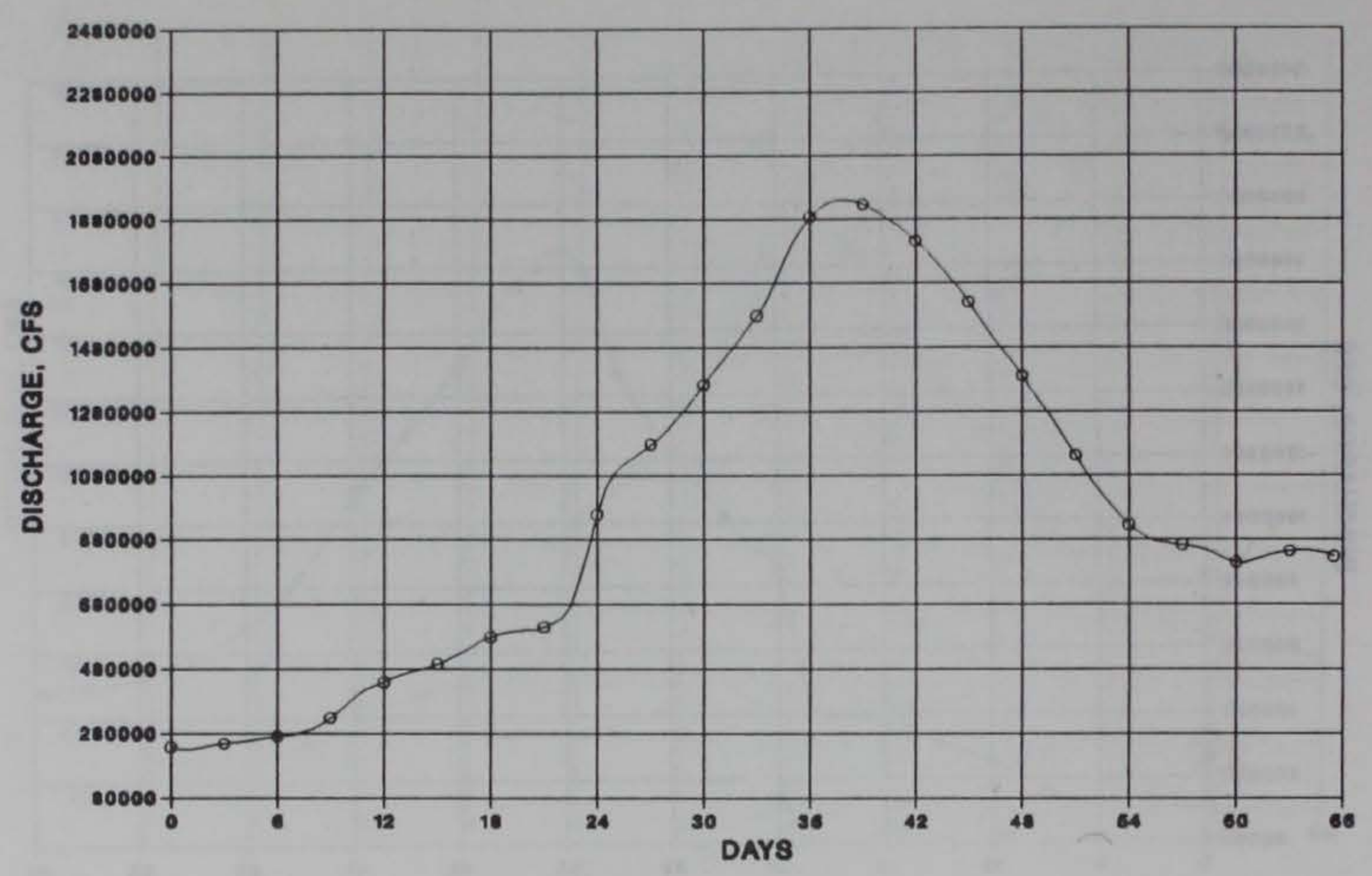

a. Discharge

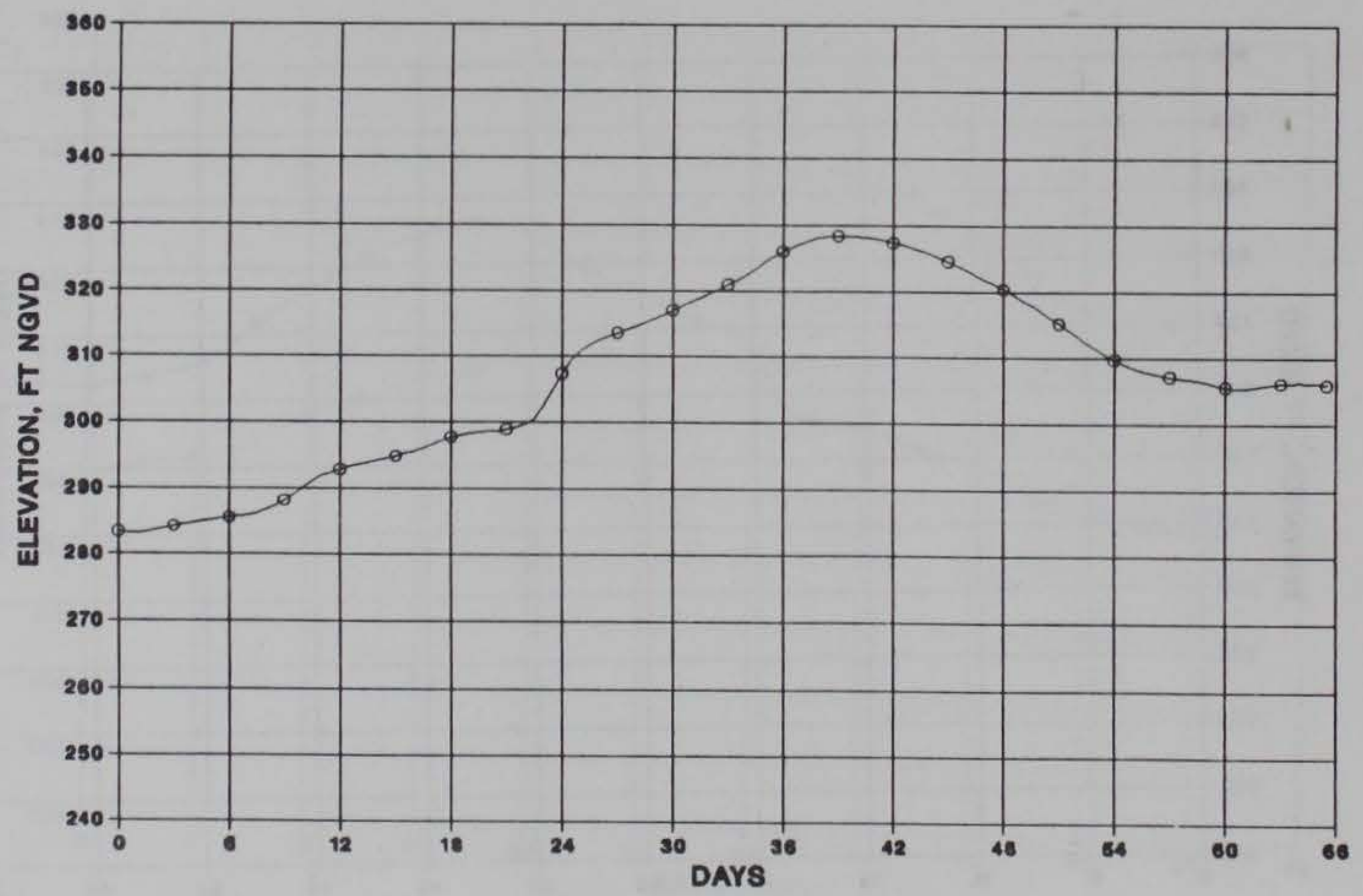

b. Elevation

Figure 20. Results at Columbus, KY, for Wilson PMF dam break 


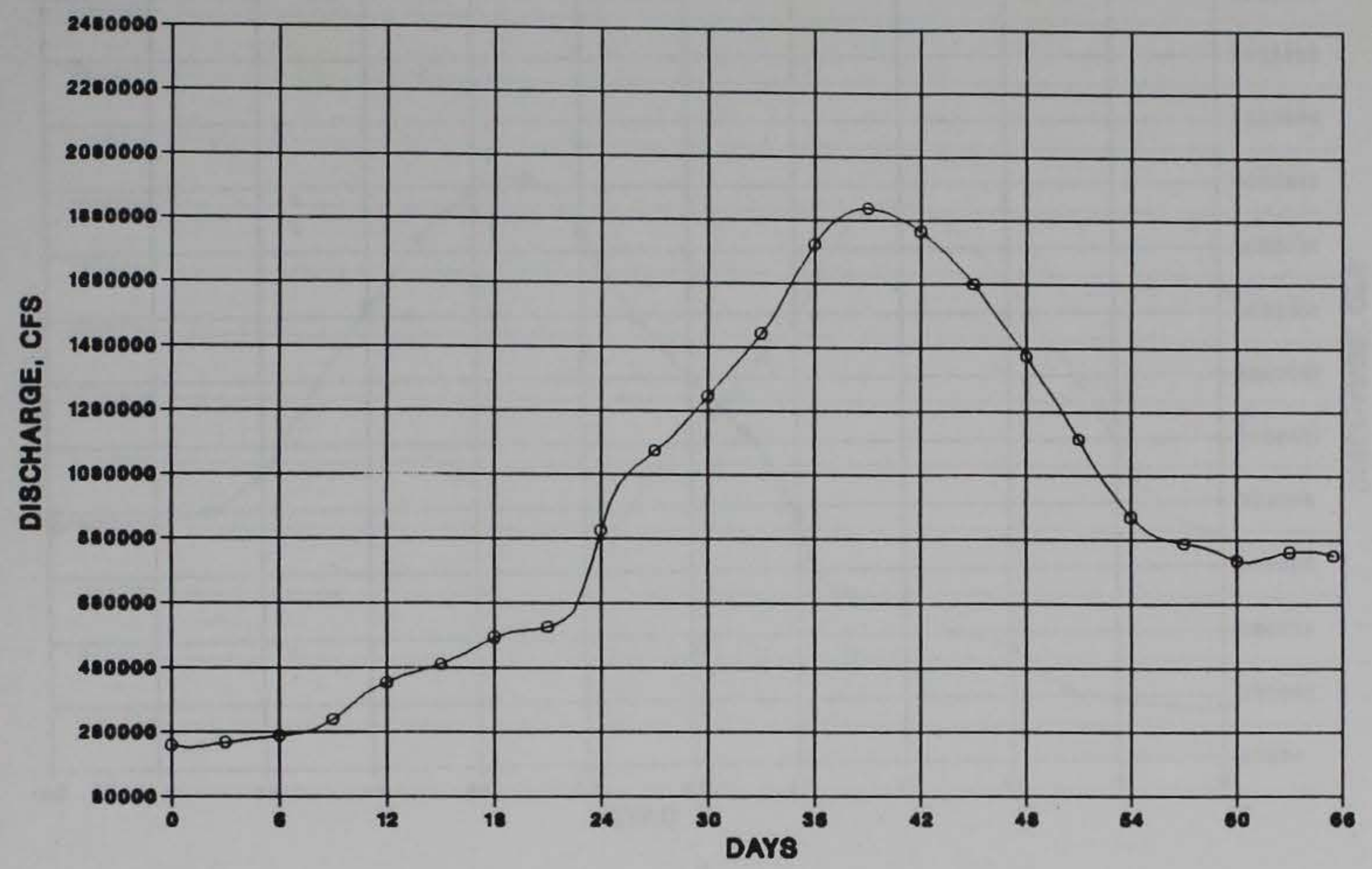

a. Discharge

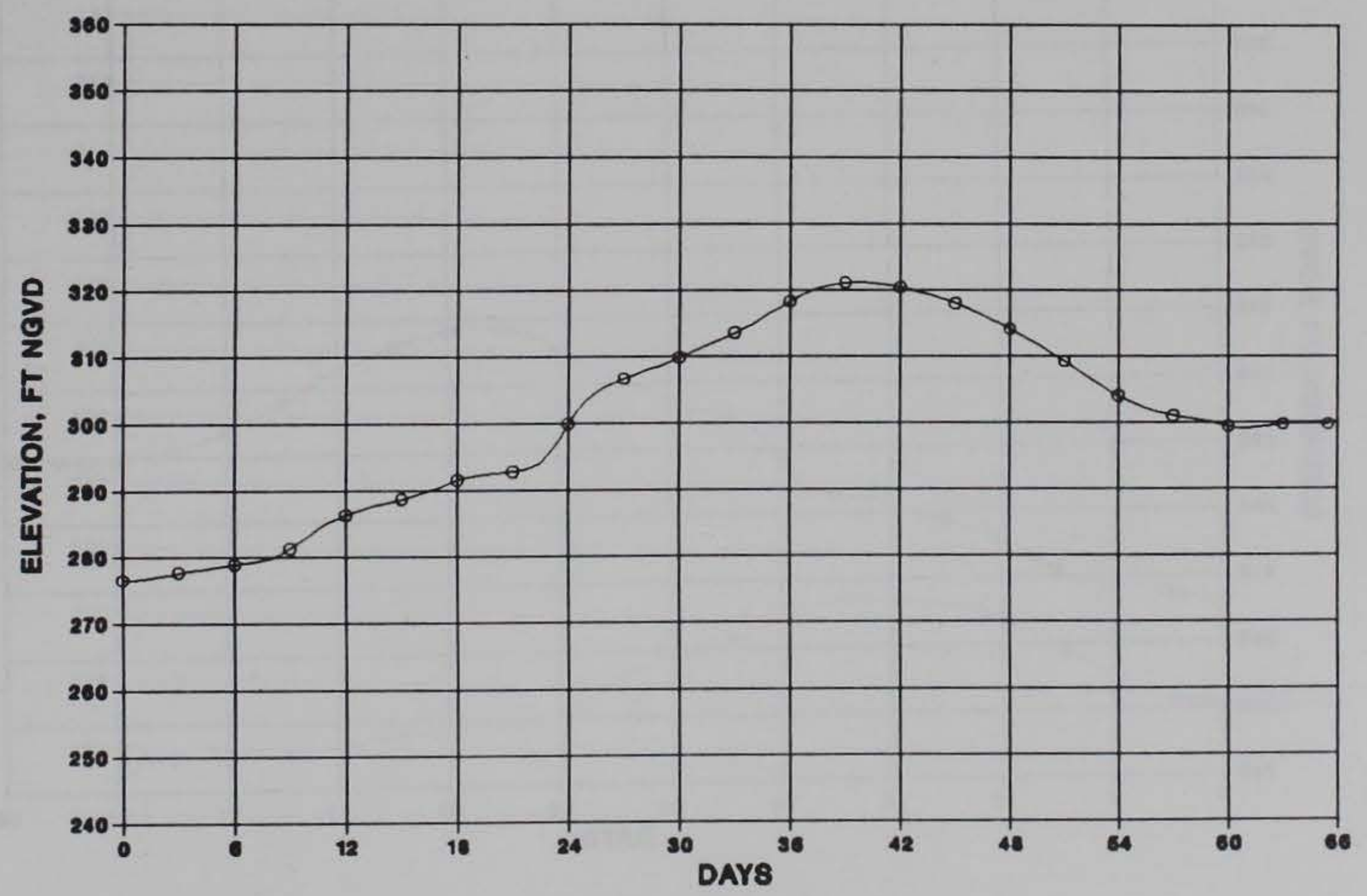

b. Elevation

Figure 21. Results at Hickman, KY, for Wilson PMF dam break 


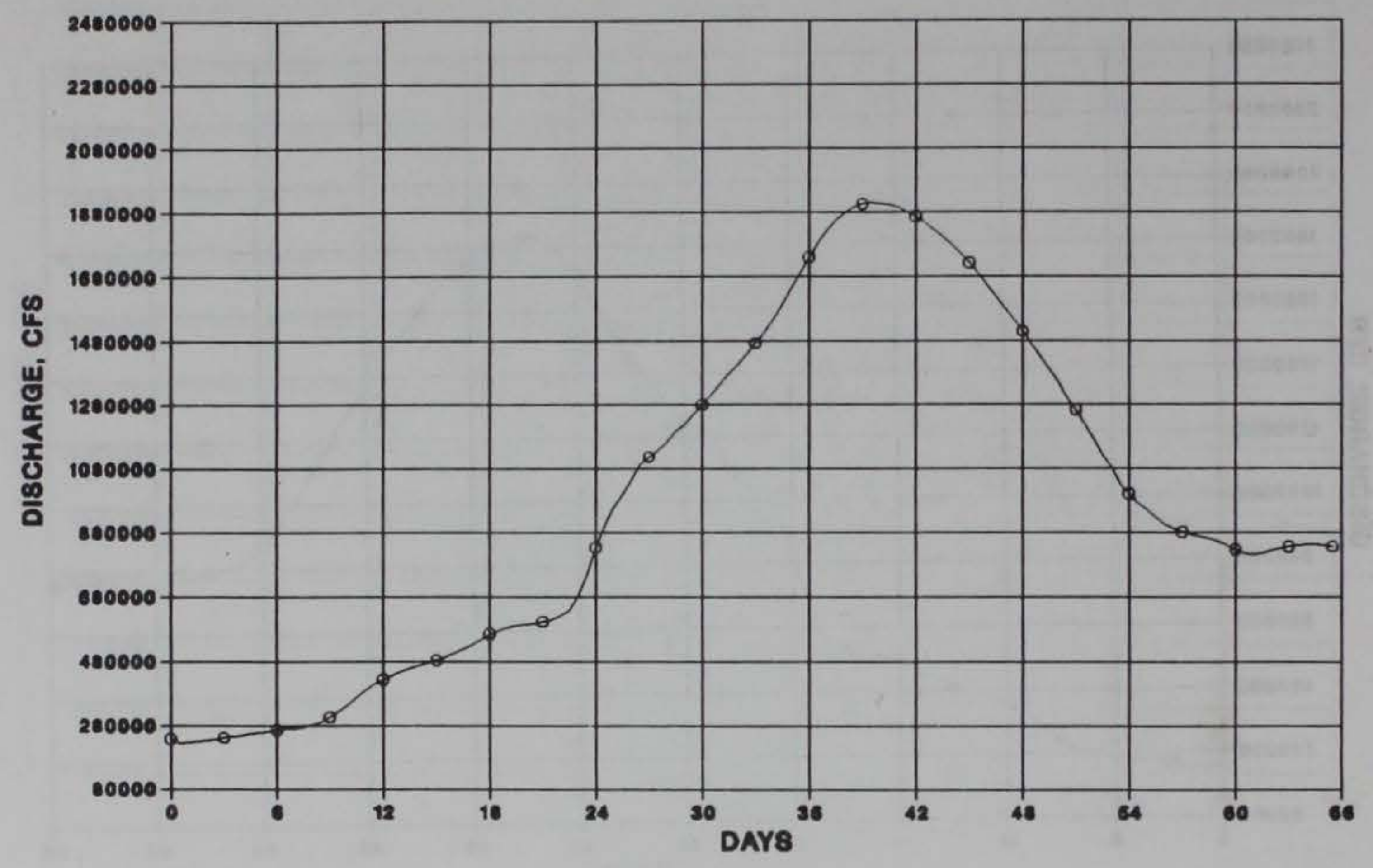

a. Discharge

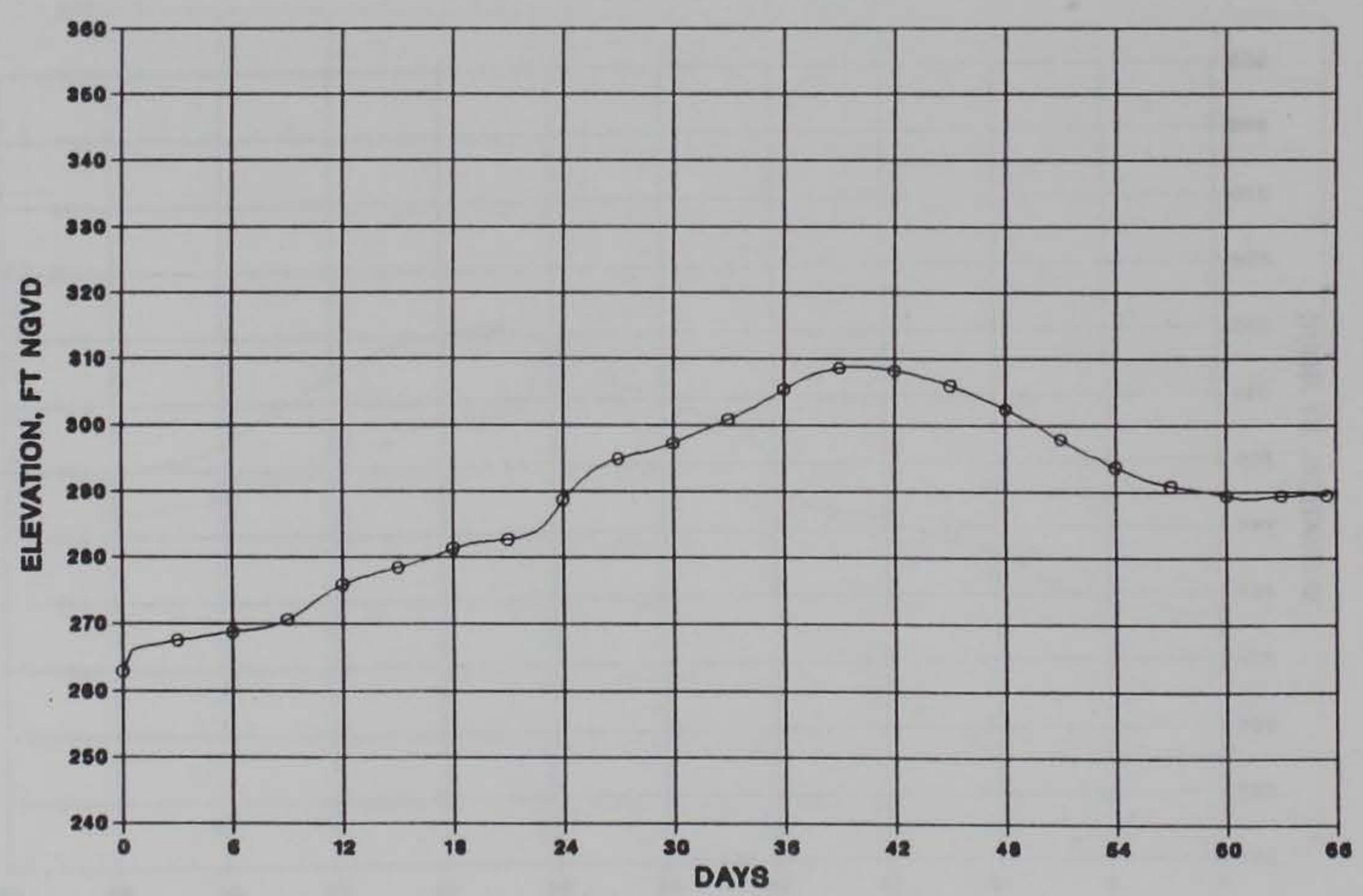

b. Elevation

Figure 22. Results at New Madrid, MO, for Wilson PMF dam break 


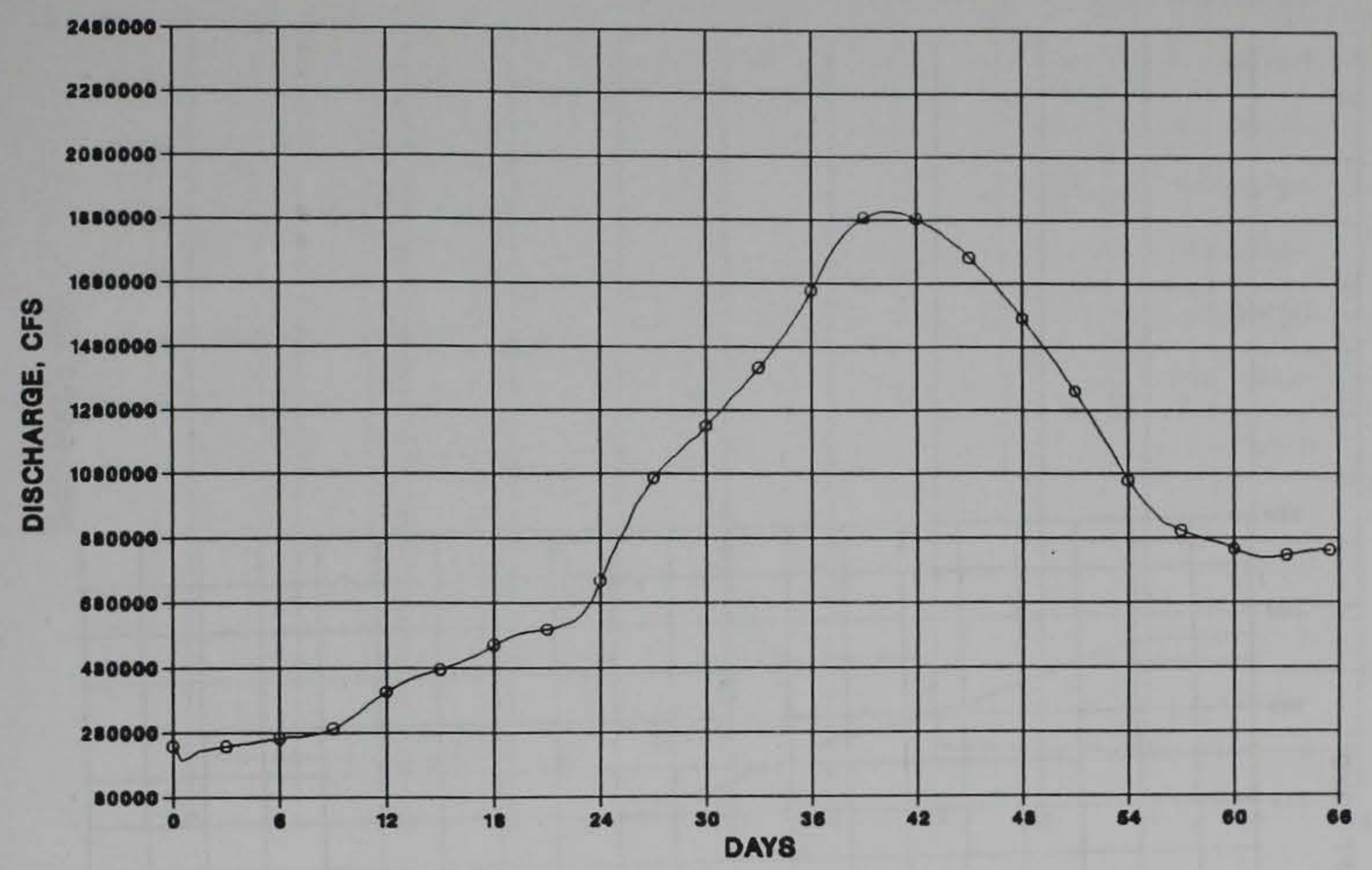

a. Discharge

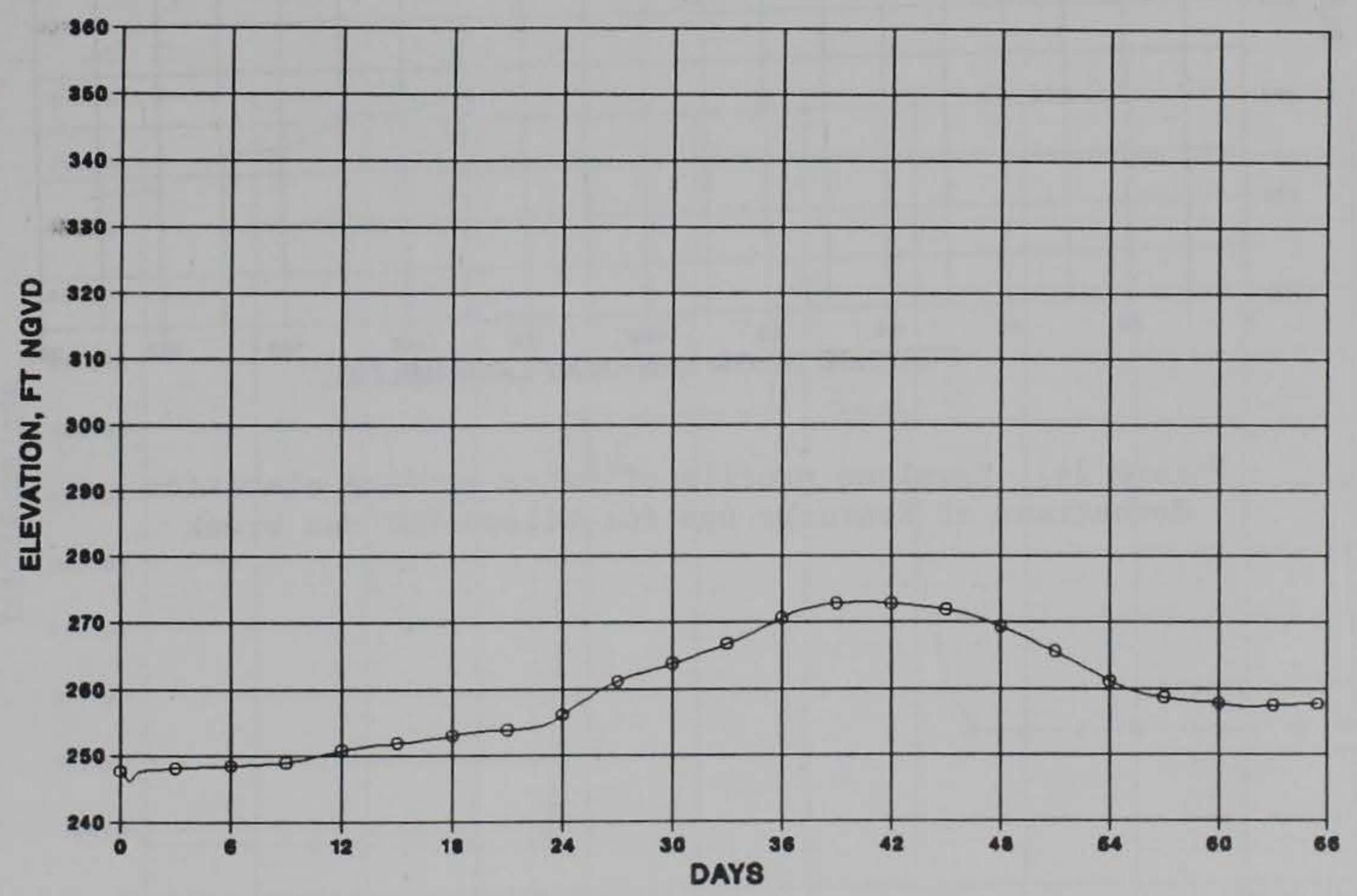

b. Elevation

Figure 23. Results at Caruthersville, MO, for Wilson PMF dam break 


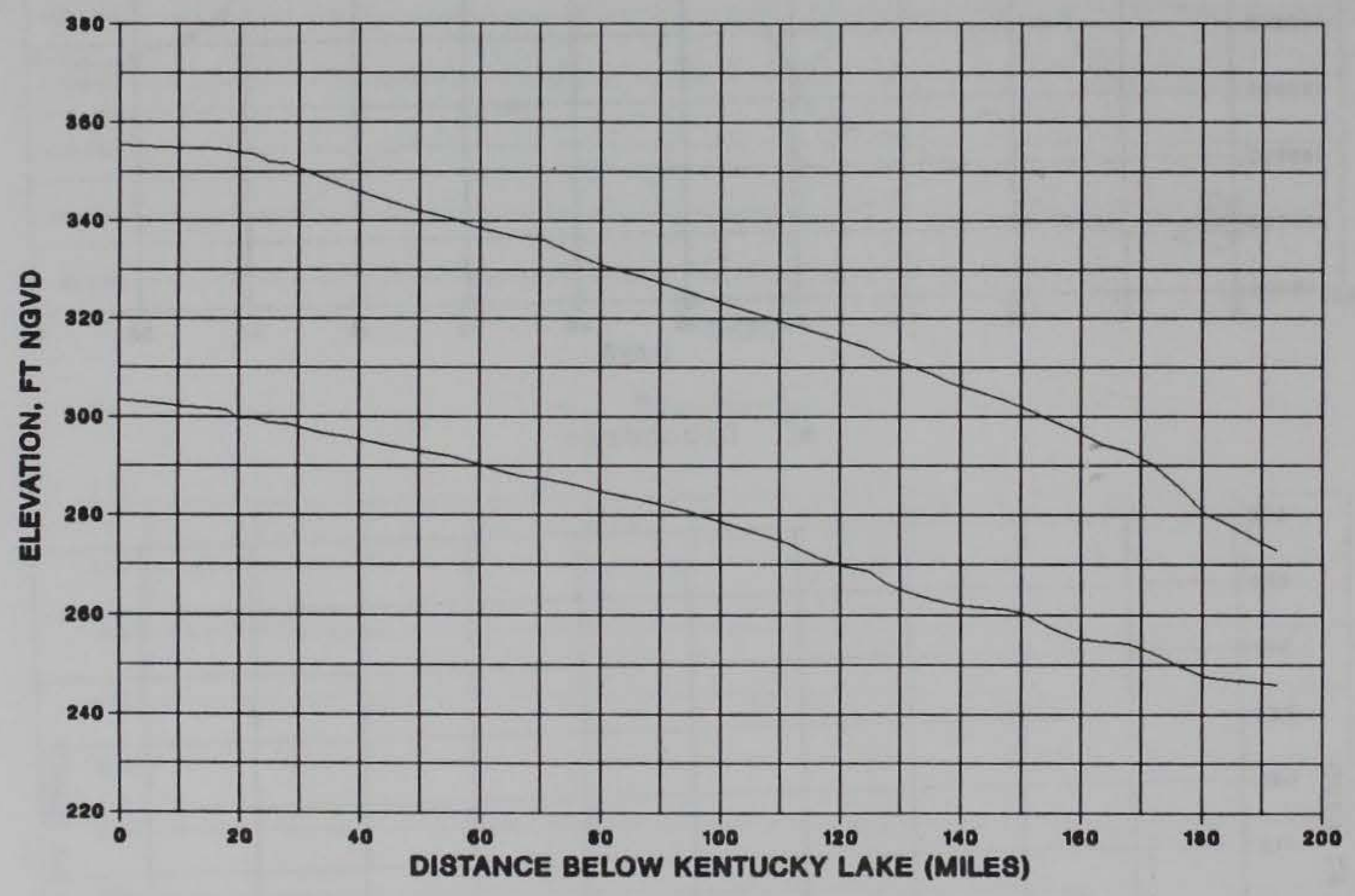

Figure 24. Envelope profile of water surface elevation downstream of Kentucky Dam for Wilson PMF dam break 


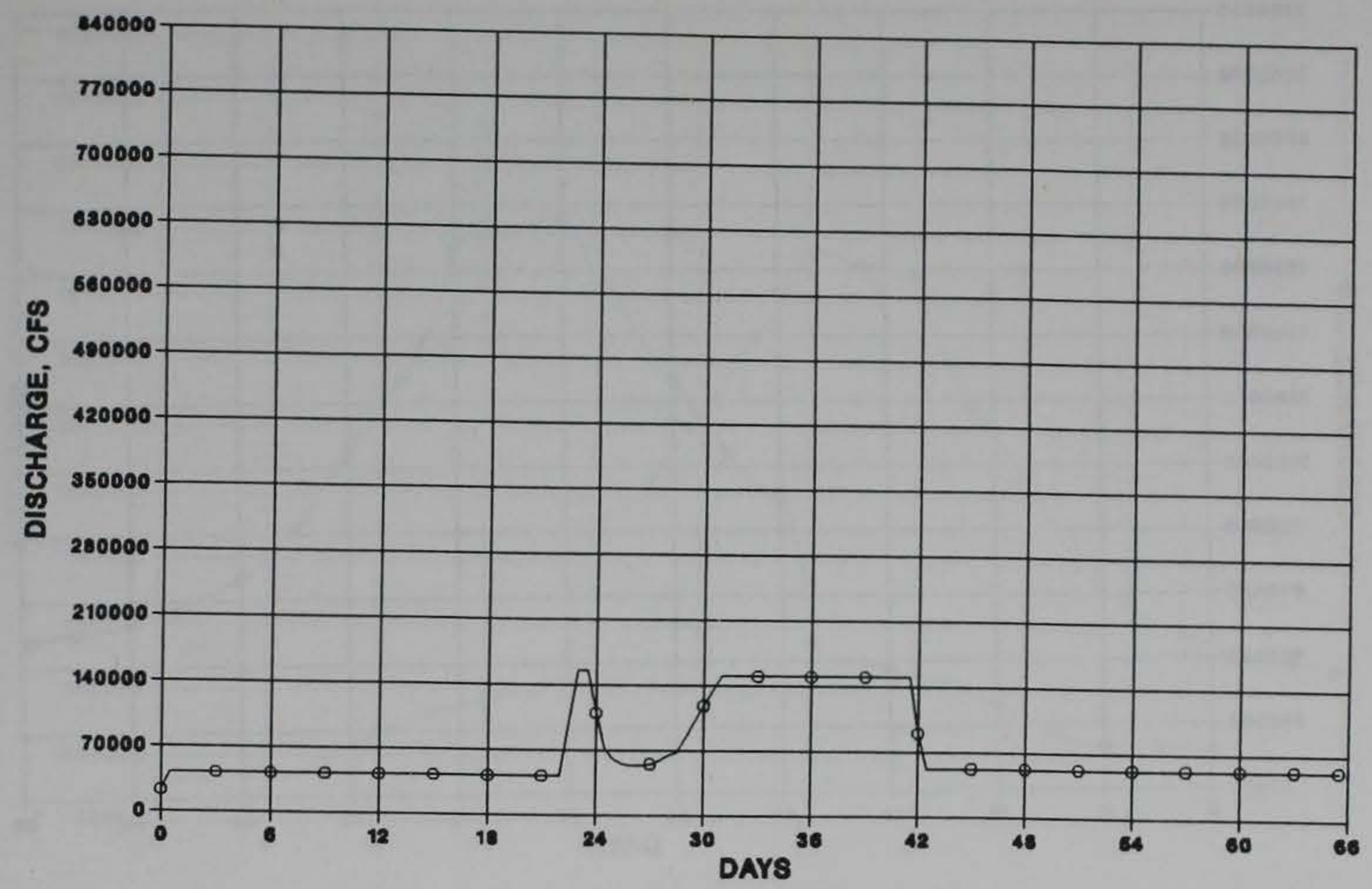

Figure 25. Discharge from Barkley Lake for Wilson PMF (Elev 380) dam break

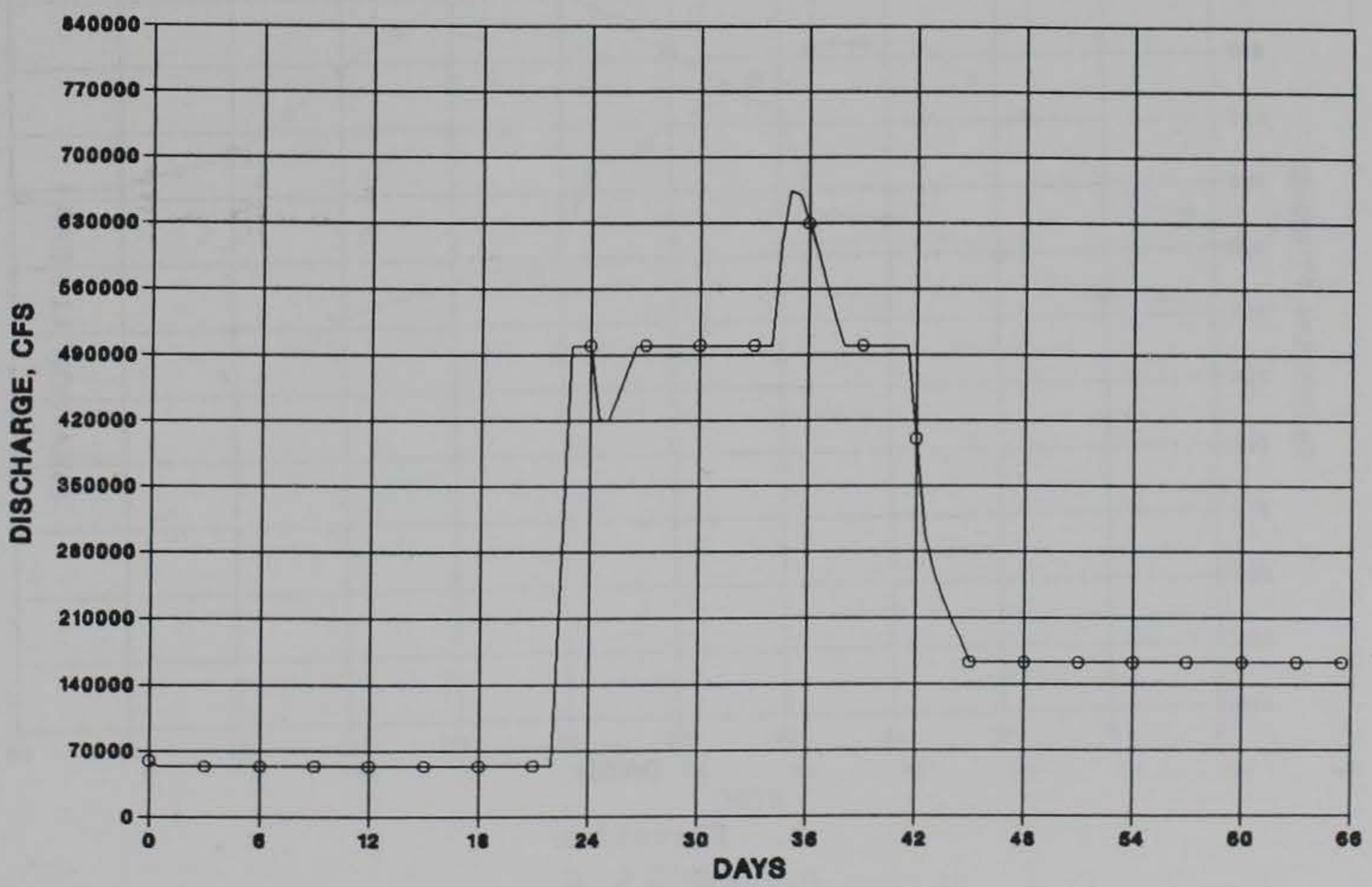

Figure 26. Discharge from Kentucky Lake for Wilson PMF (Elev 380) dam break 


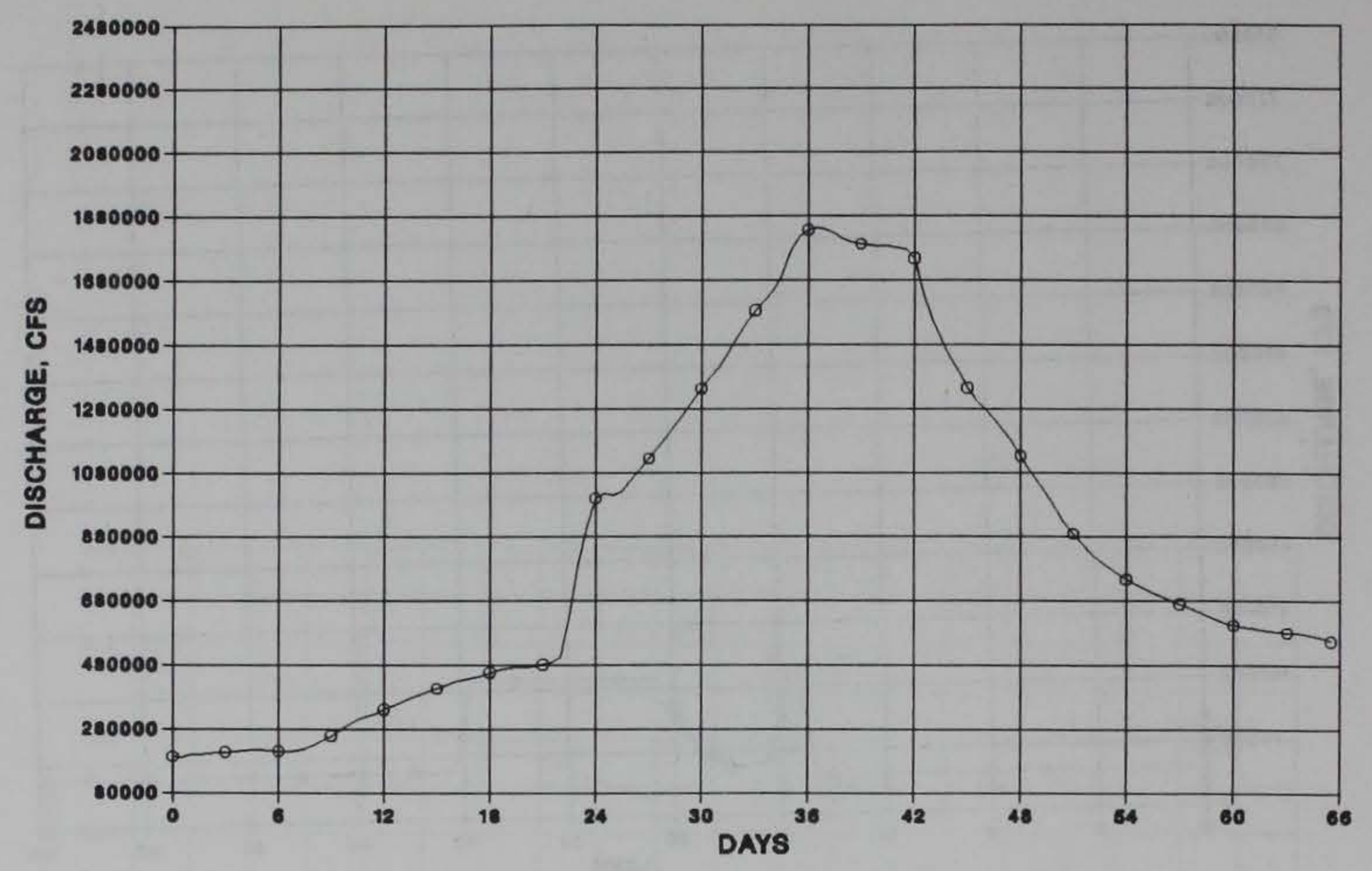

a. Discharge

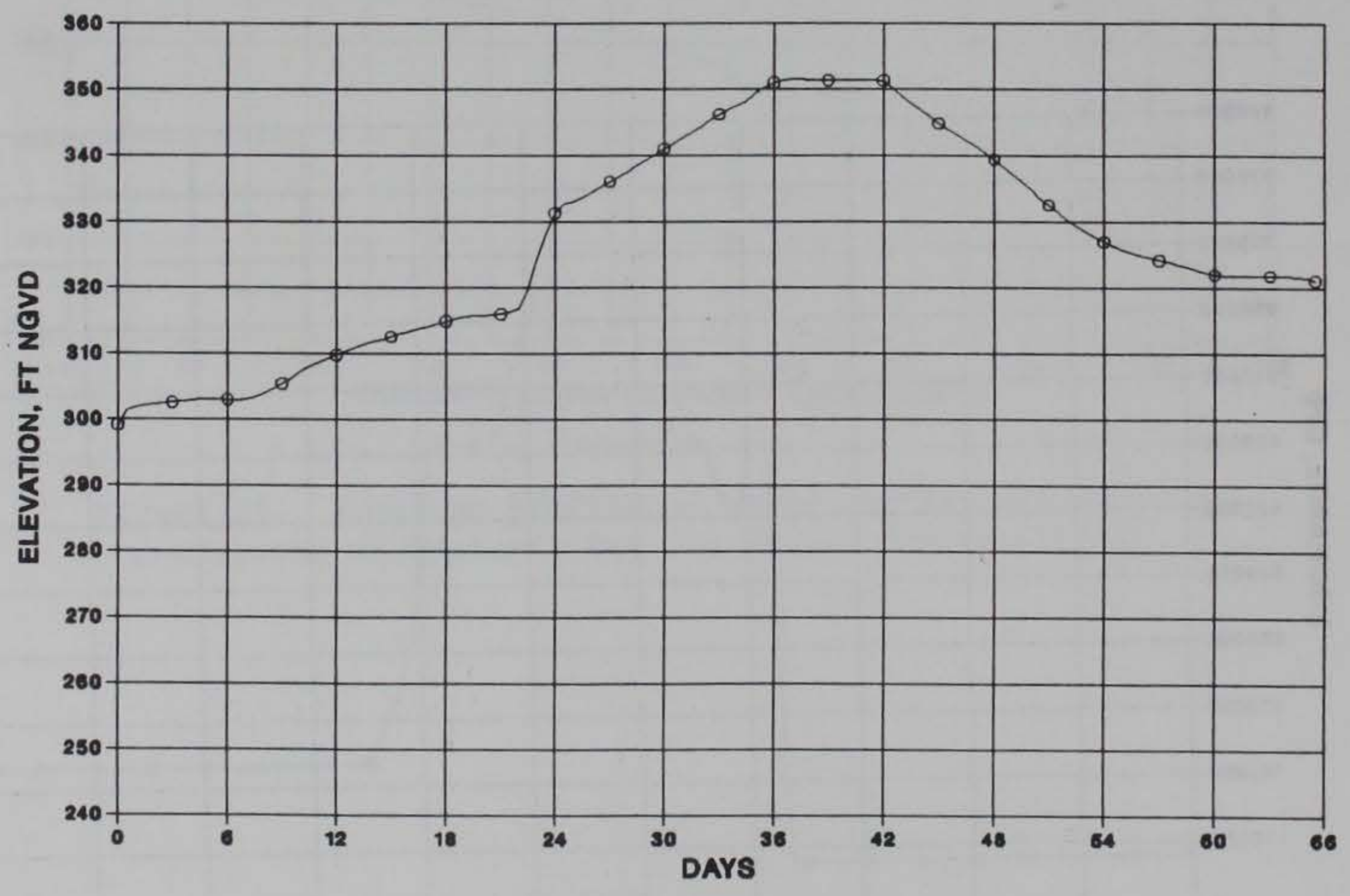

b. Elevation

Figure 27. Results at Paducah, KY, for Wilson PMF (Elev 380) dam break 


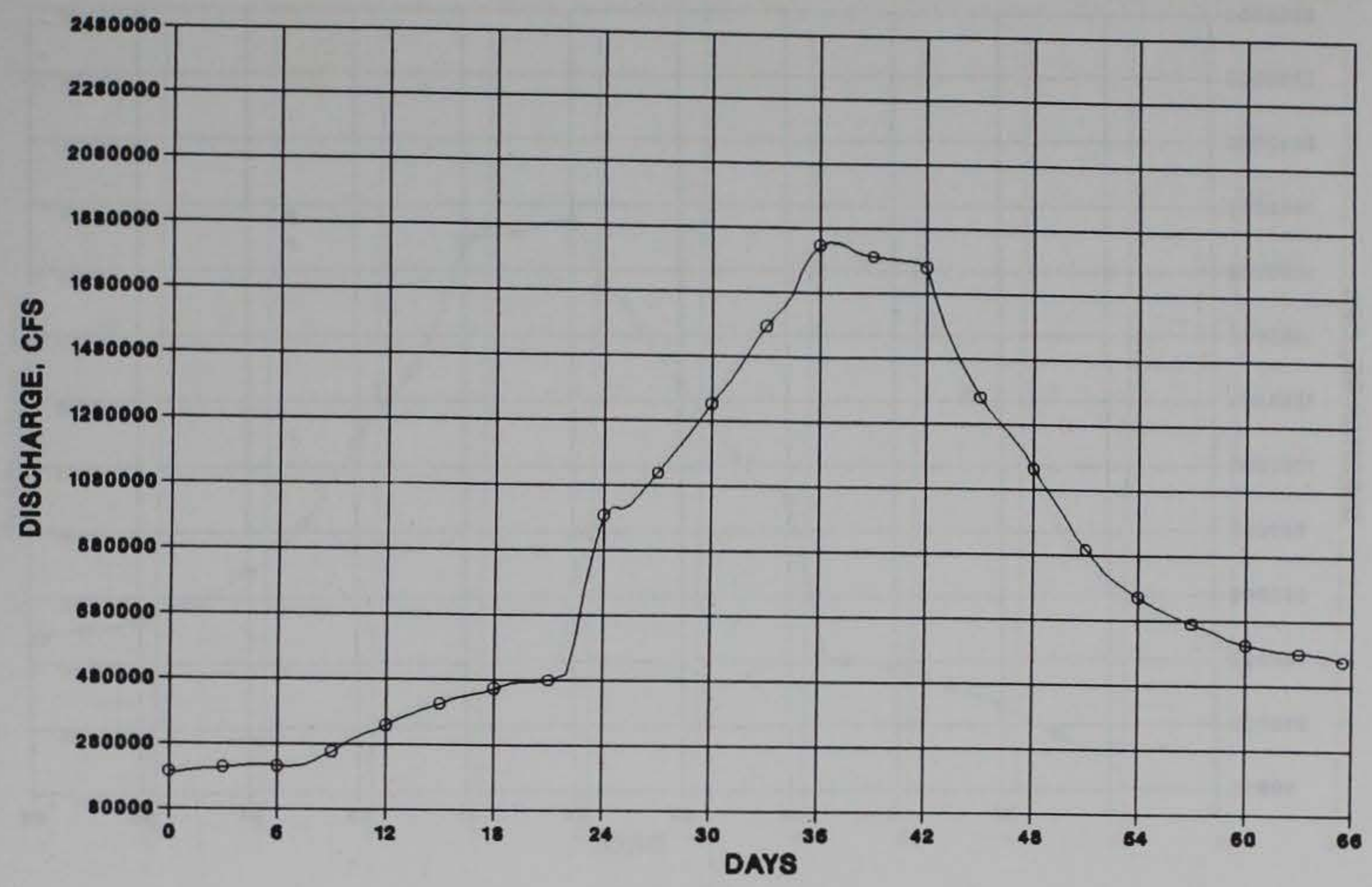

a. Discharge

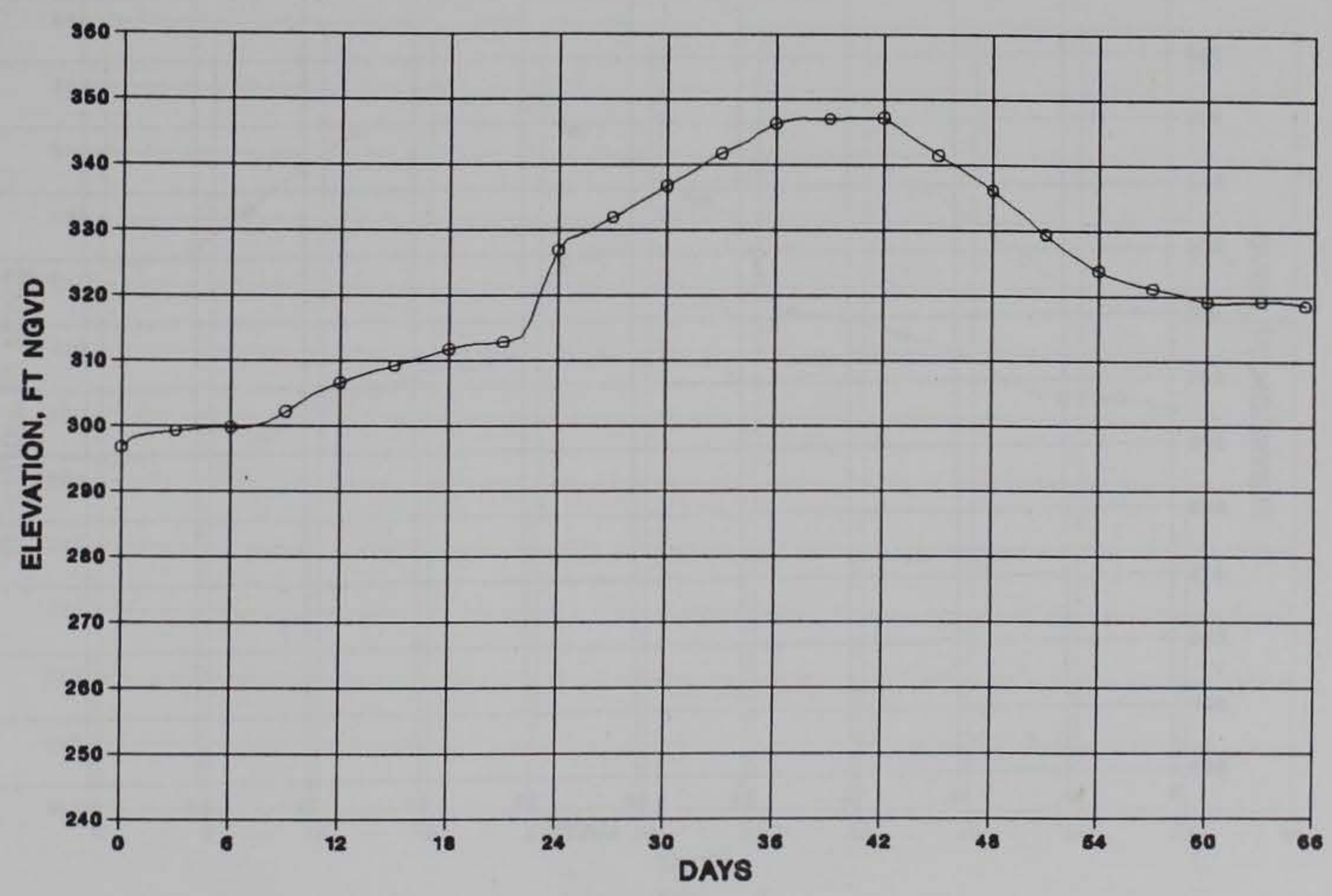

b. Elevation

Figure 28. Results at Metropolis, IL, for Wilson PMF (Elev 380) dam break 


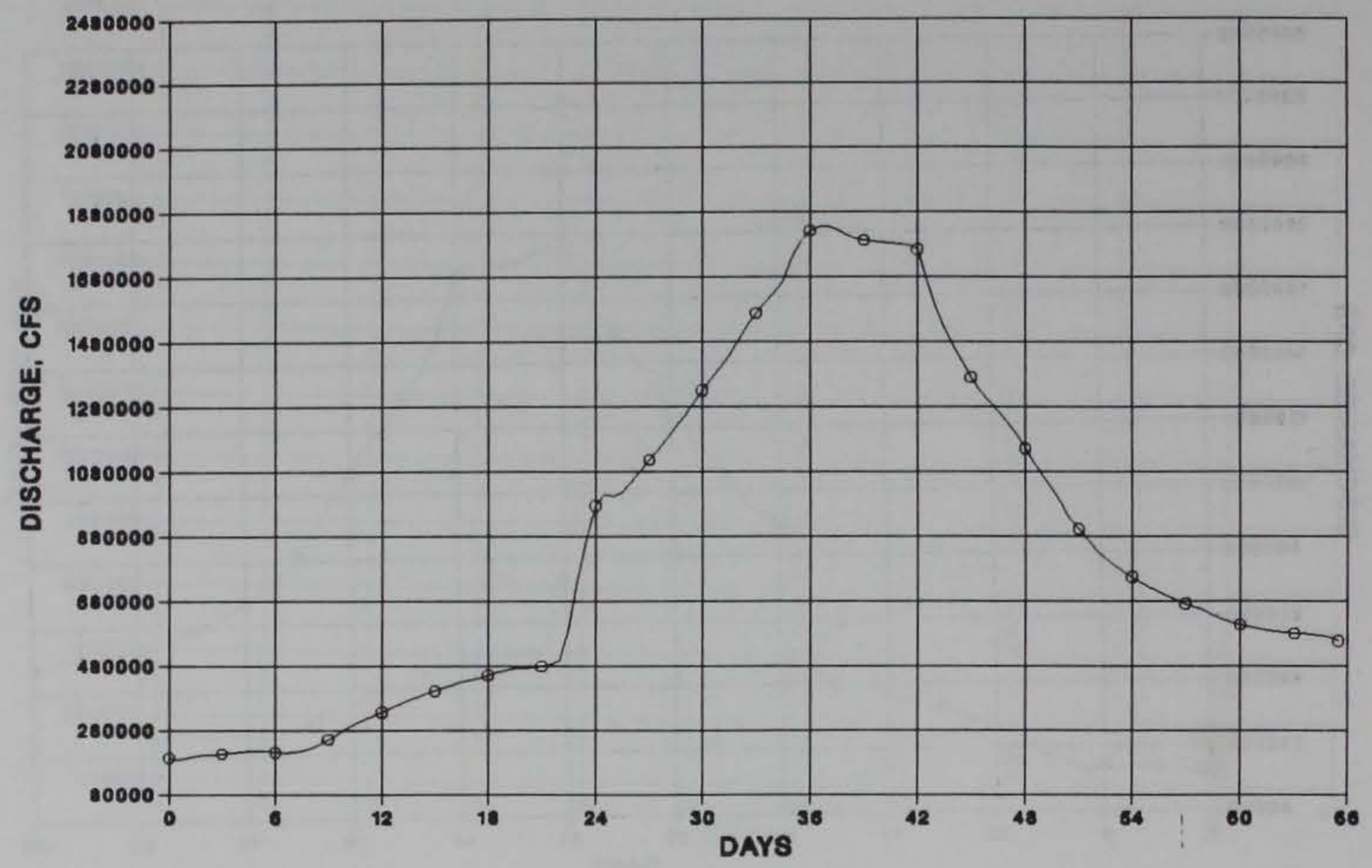

a. Discharge

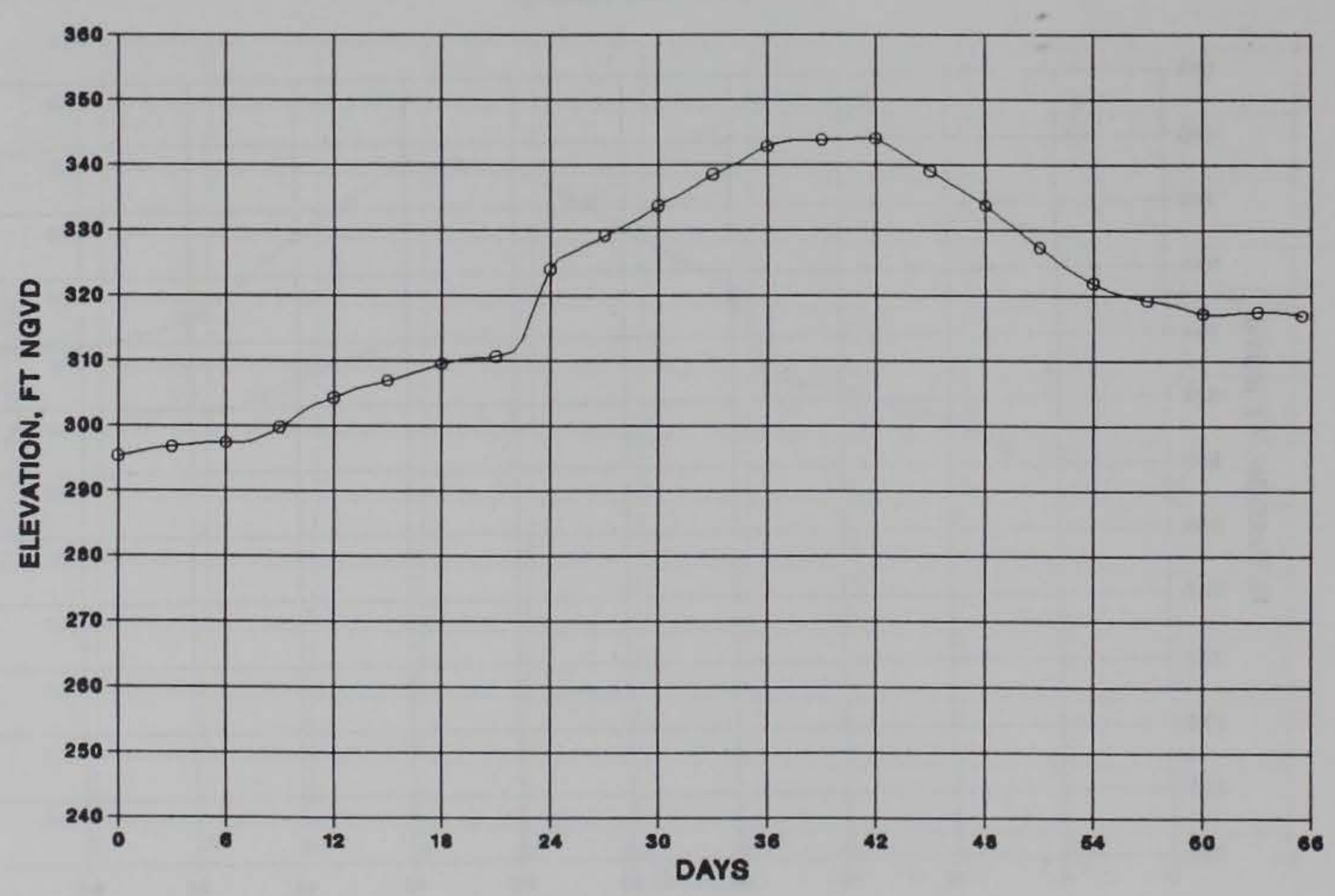

b. Elevation

Figure 29. Results at Joppa, IL, for Wilson PMF (Elev 380) dam break 


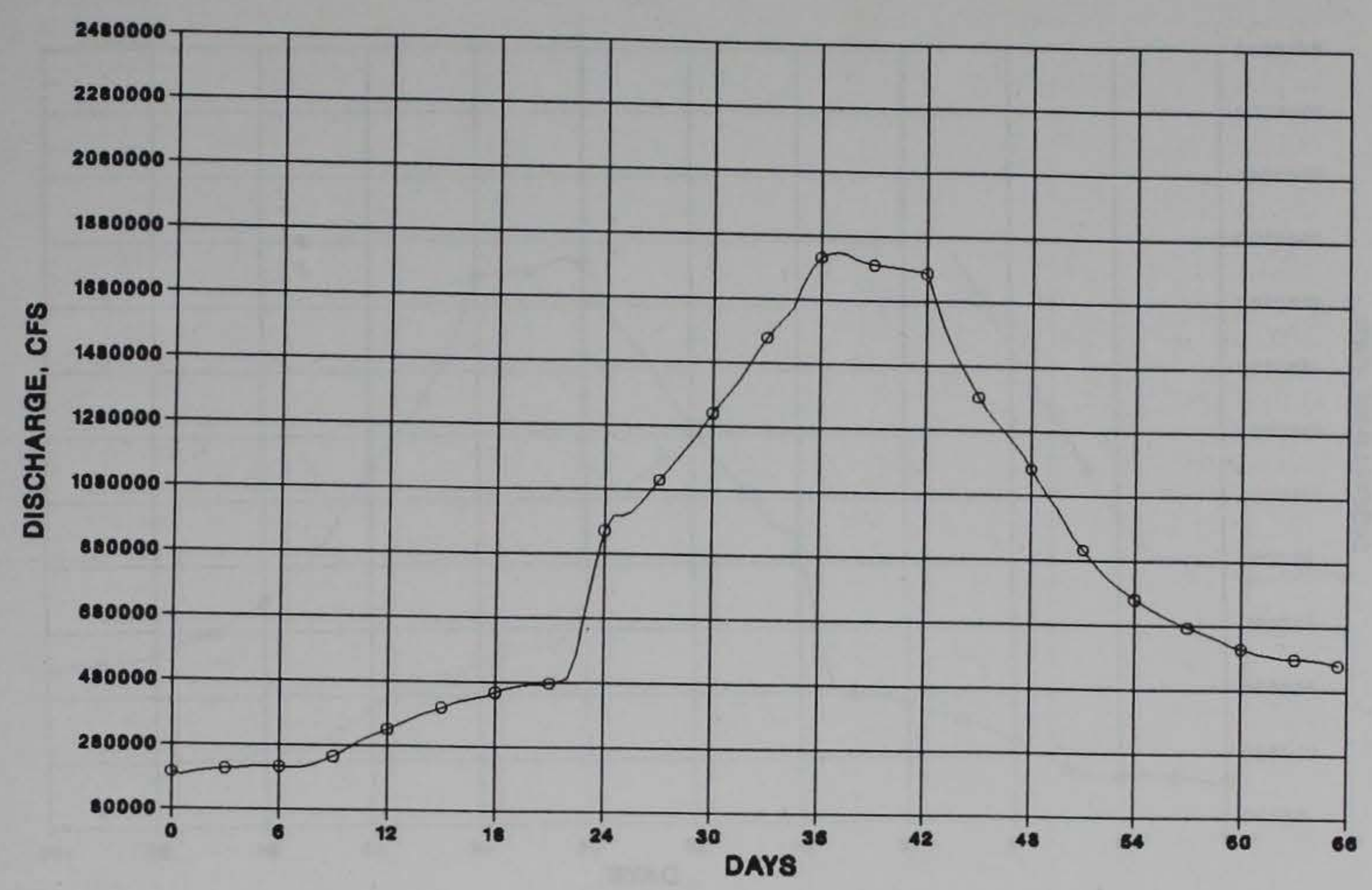

a. Discharge

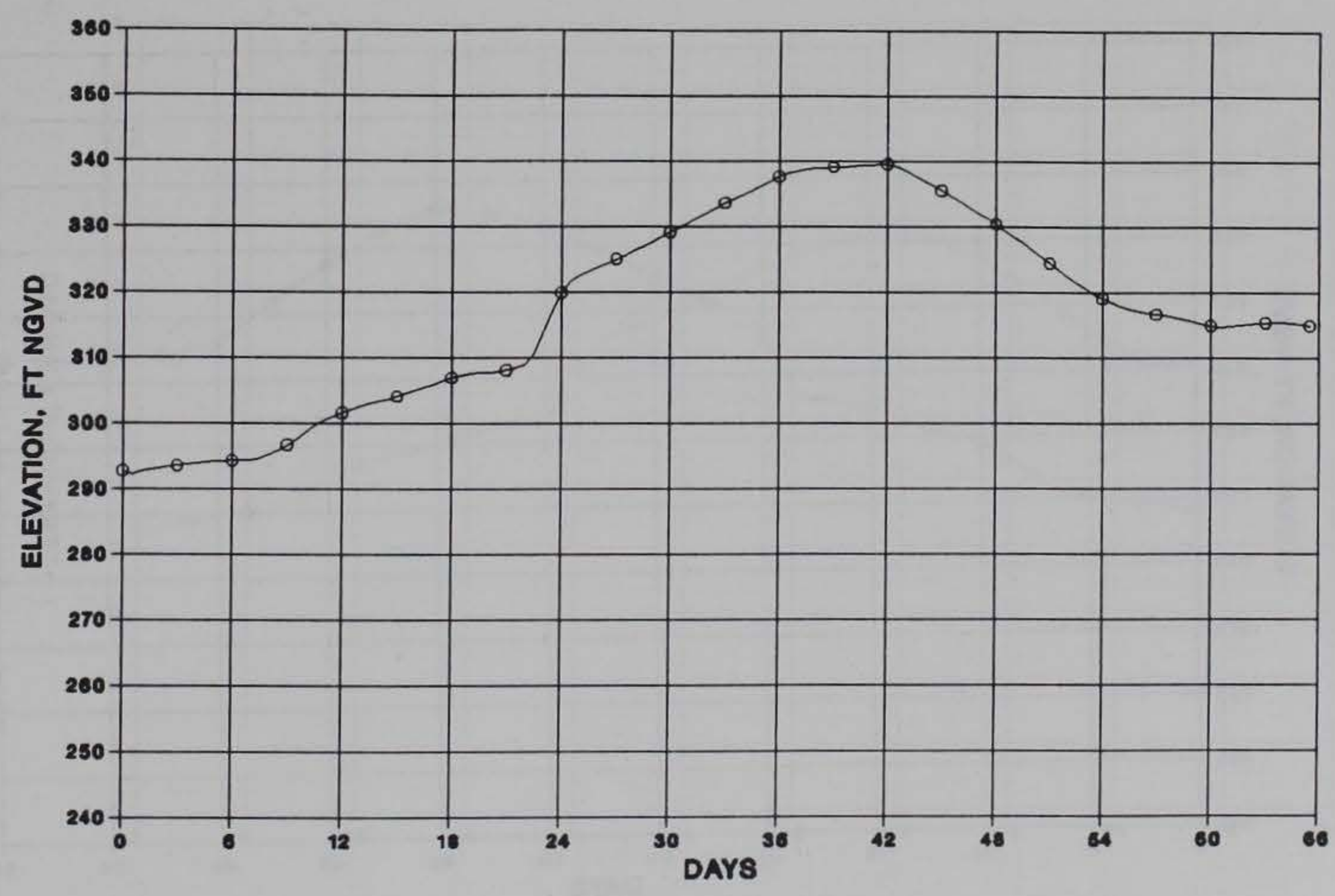

b. Elevation

Figure 30. Results at 0lmstead, IL, for Wilson PMF (Elev 380) dam break 


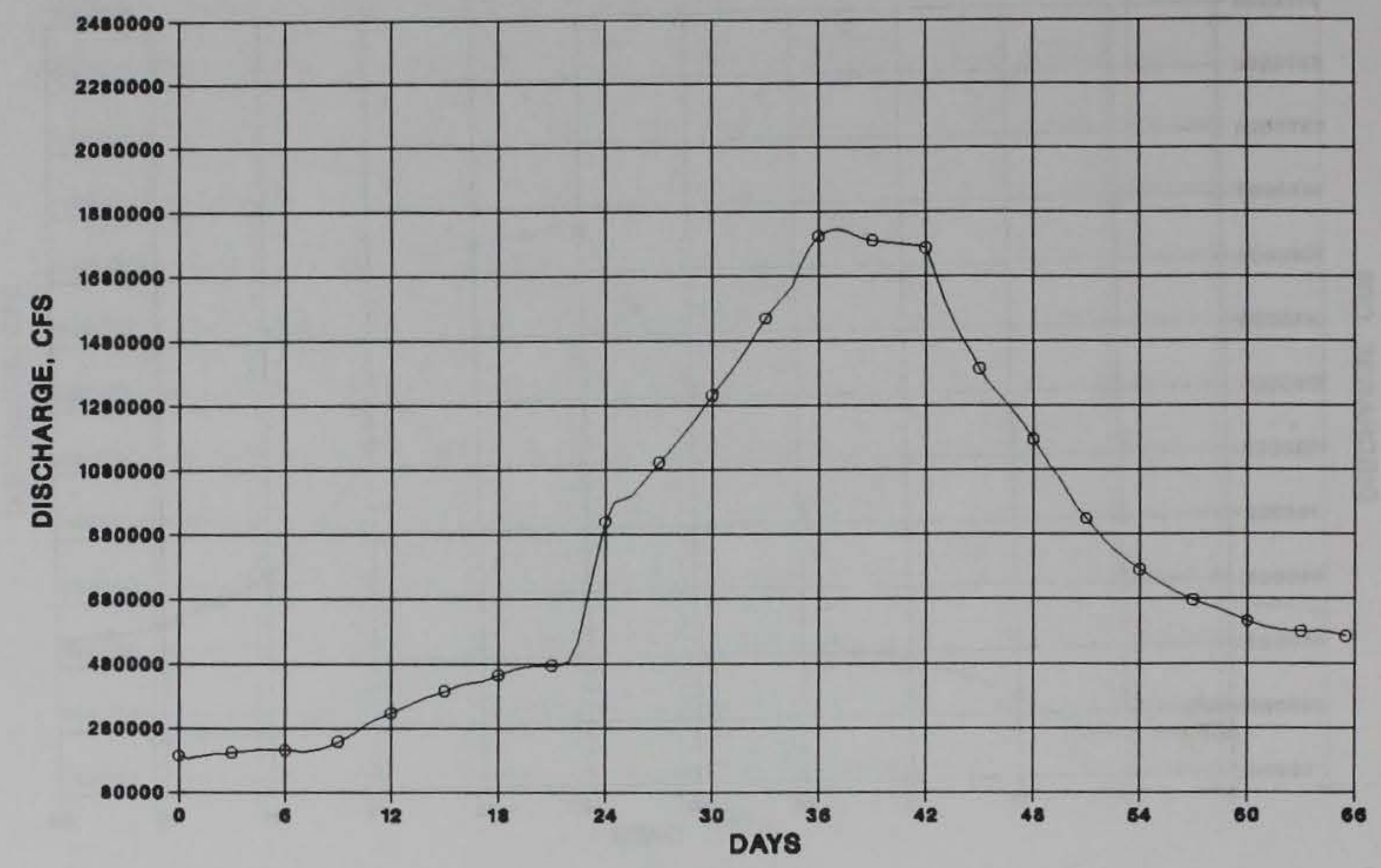

a. Discharge

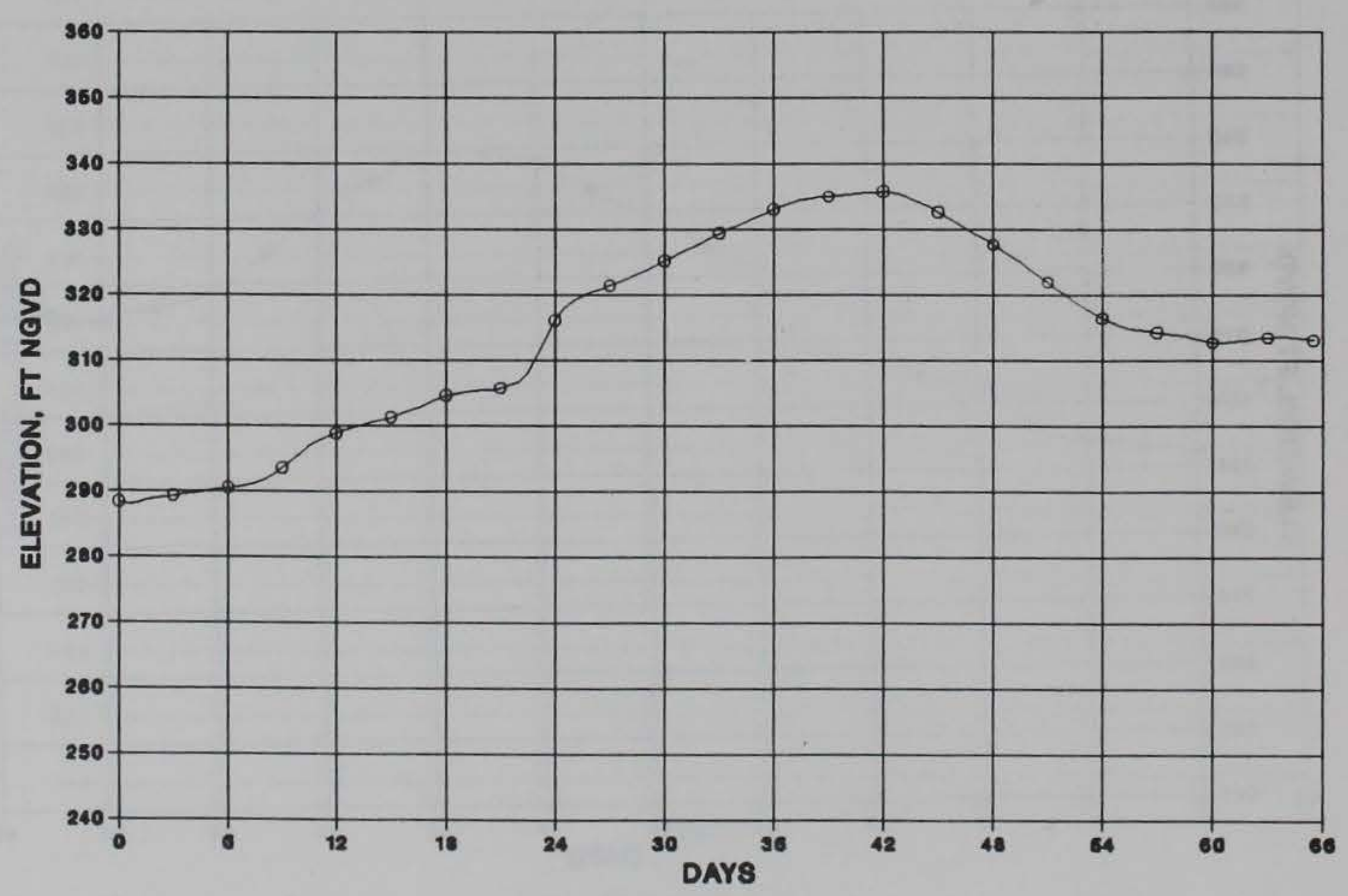

b. Elevation

Figure 31. Results at Cairo, IL, for Wilson PMF (Elev 380) dam break 


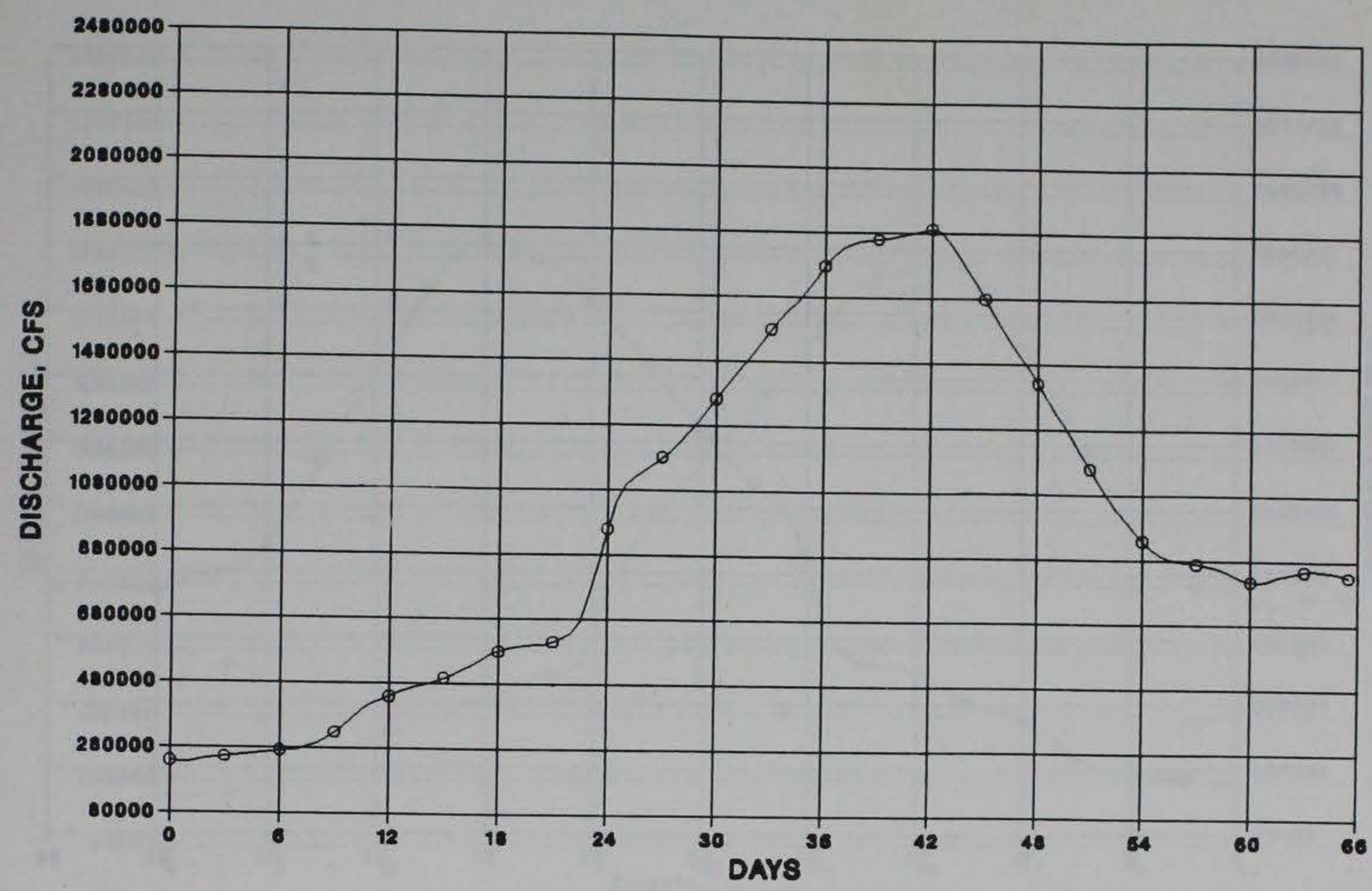

a. Discharge

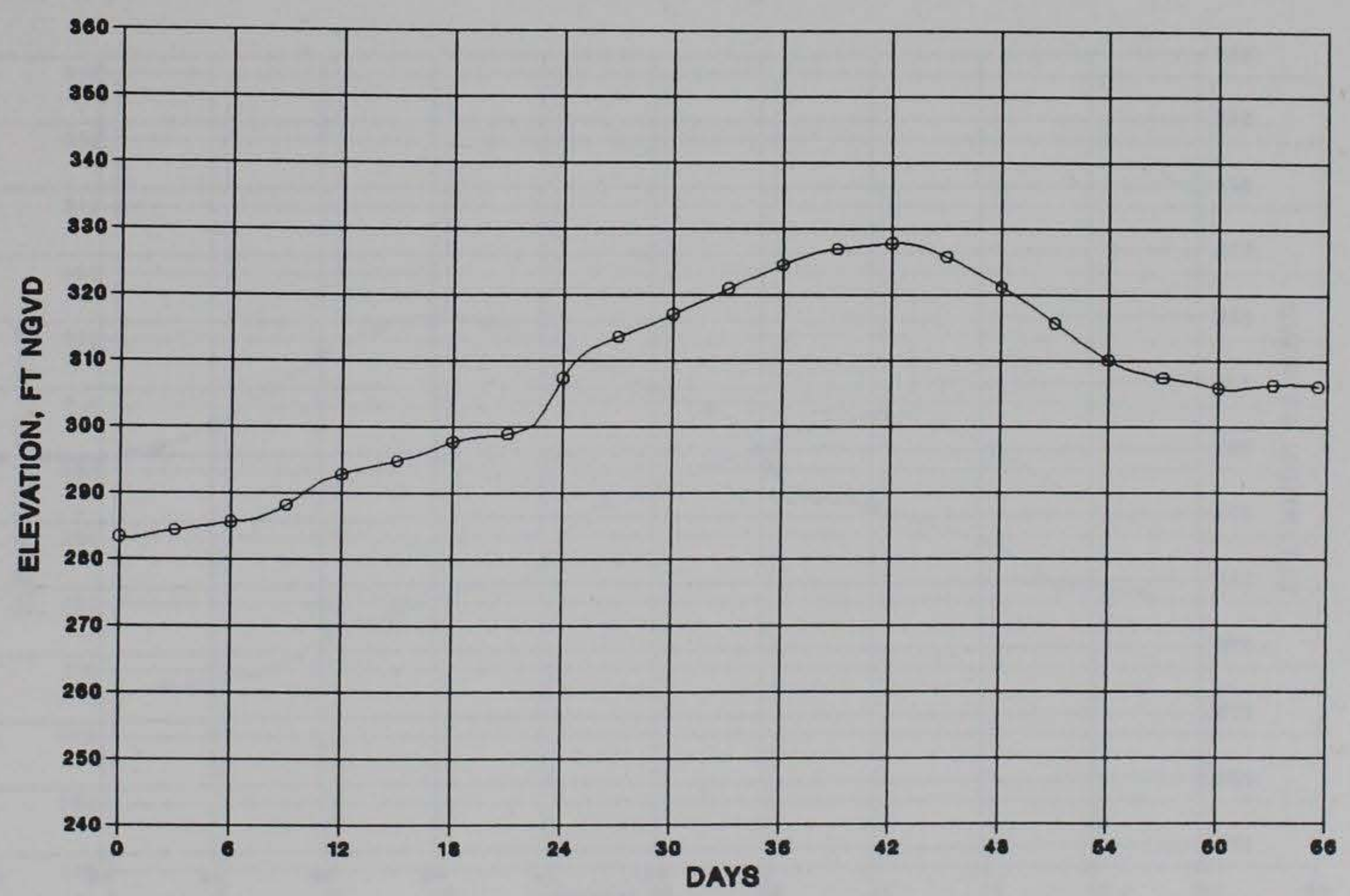

b. Elevation

Figure 32. Results at Columbus, KY, for Wilson PMF (Elev 380) dam break 


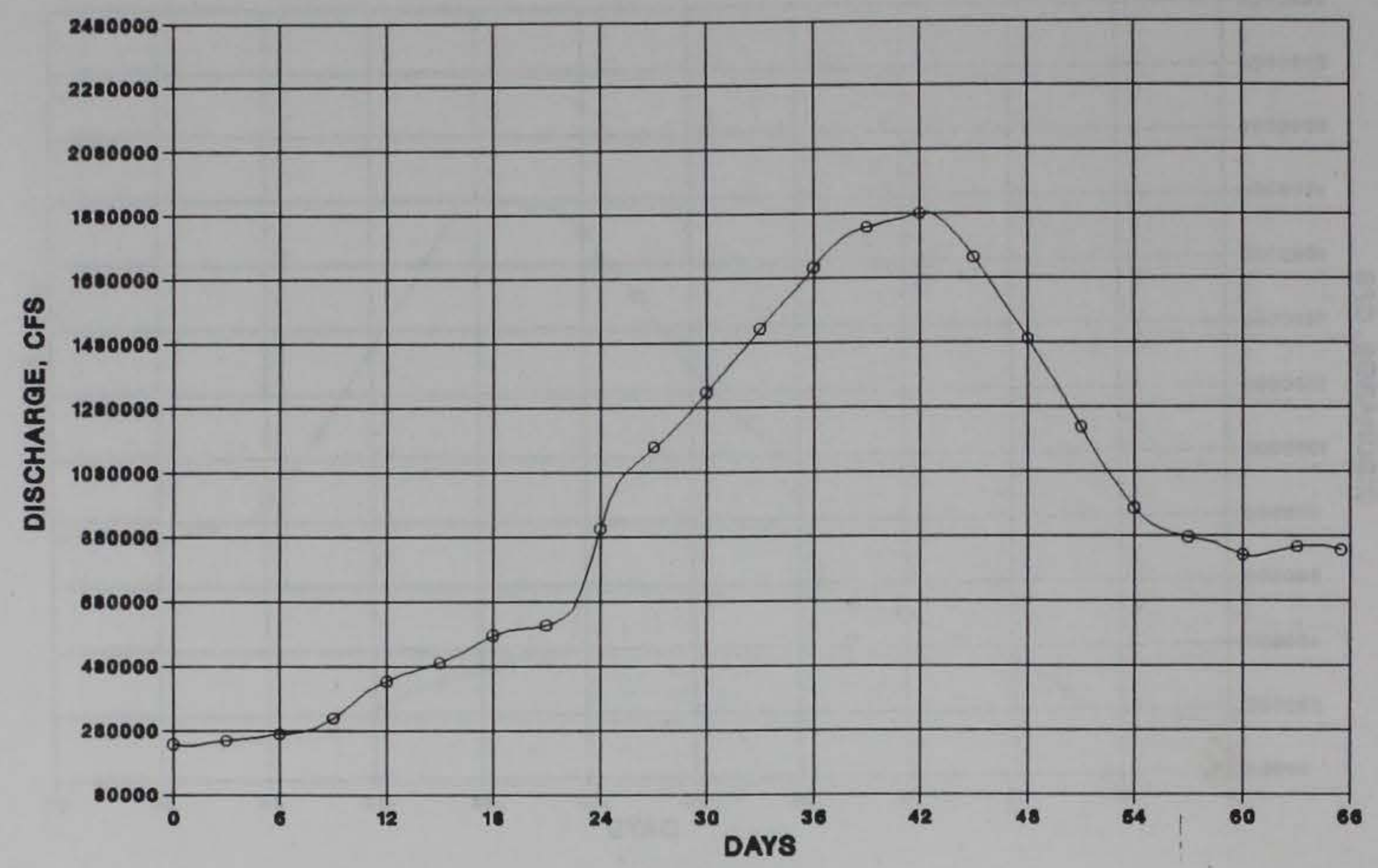

a. Discharge

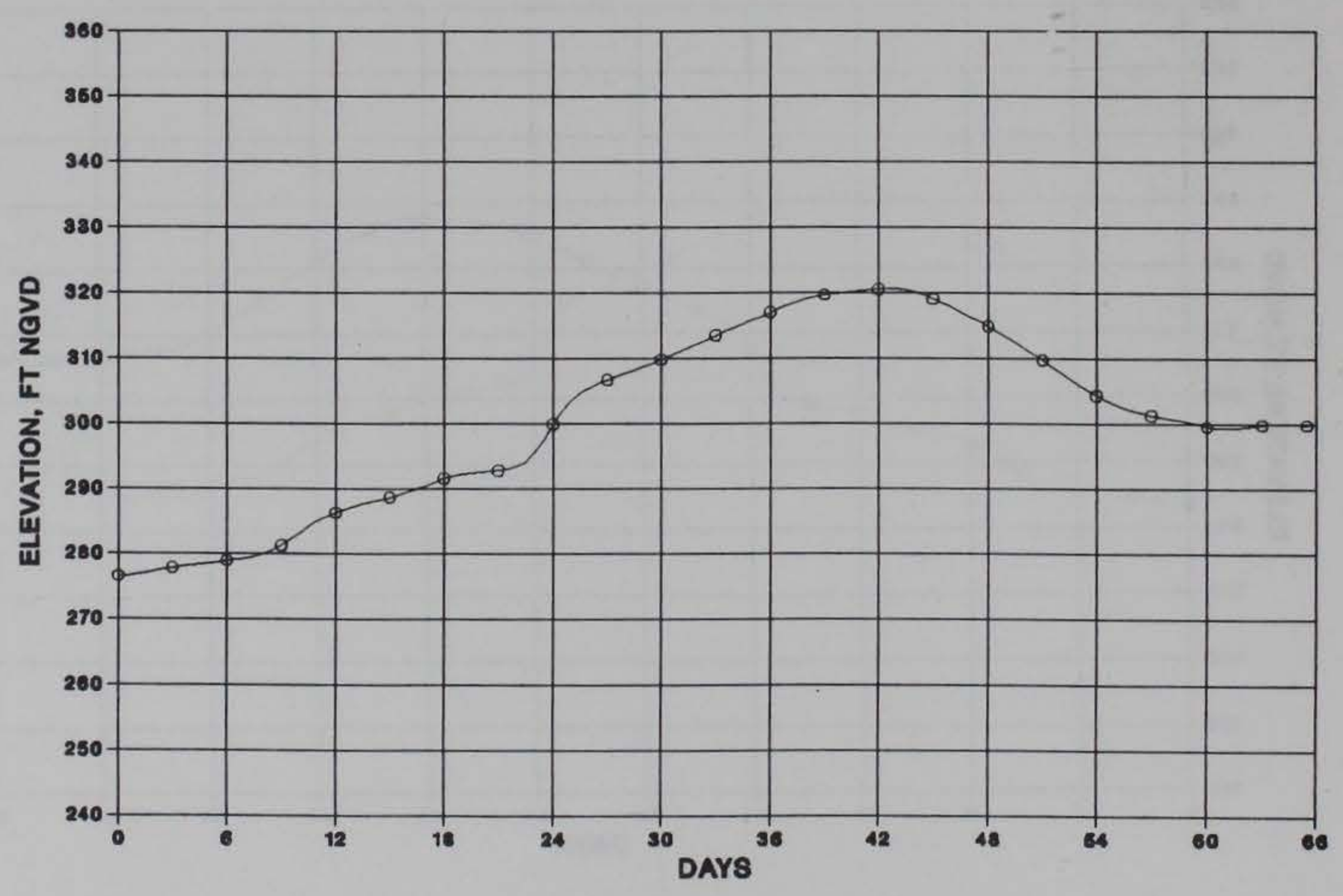

b. Elevation

Figure 33. Results at Hickman, KY, for Wilson PMF (Elev 380) dam break 


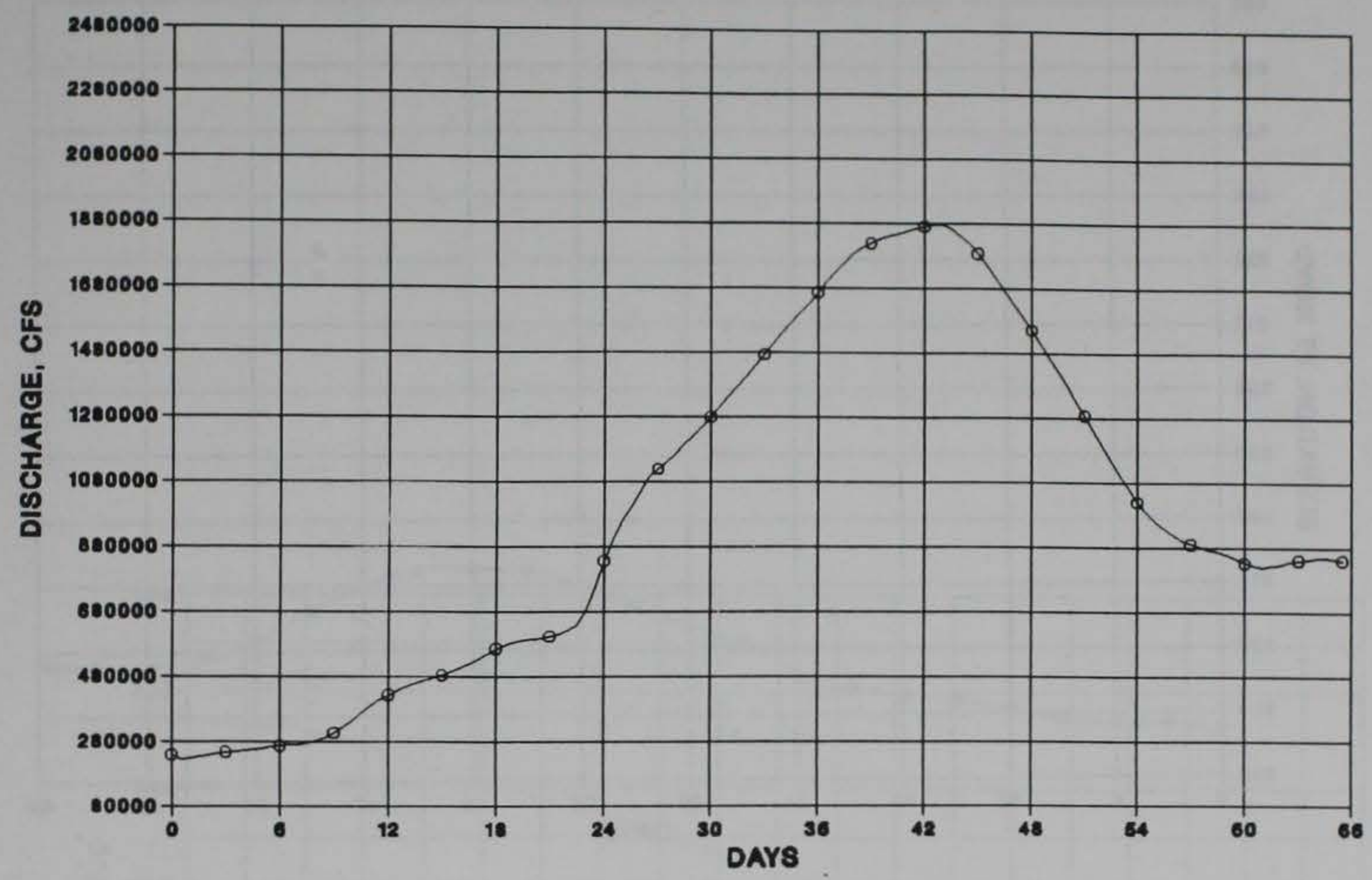

a. Discharge

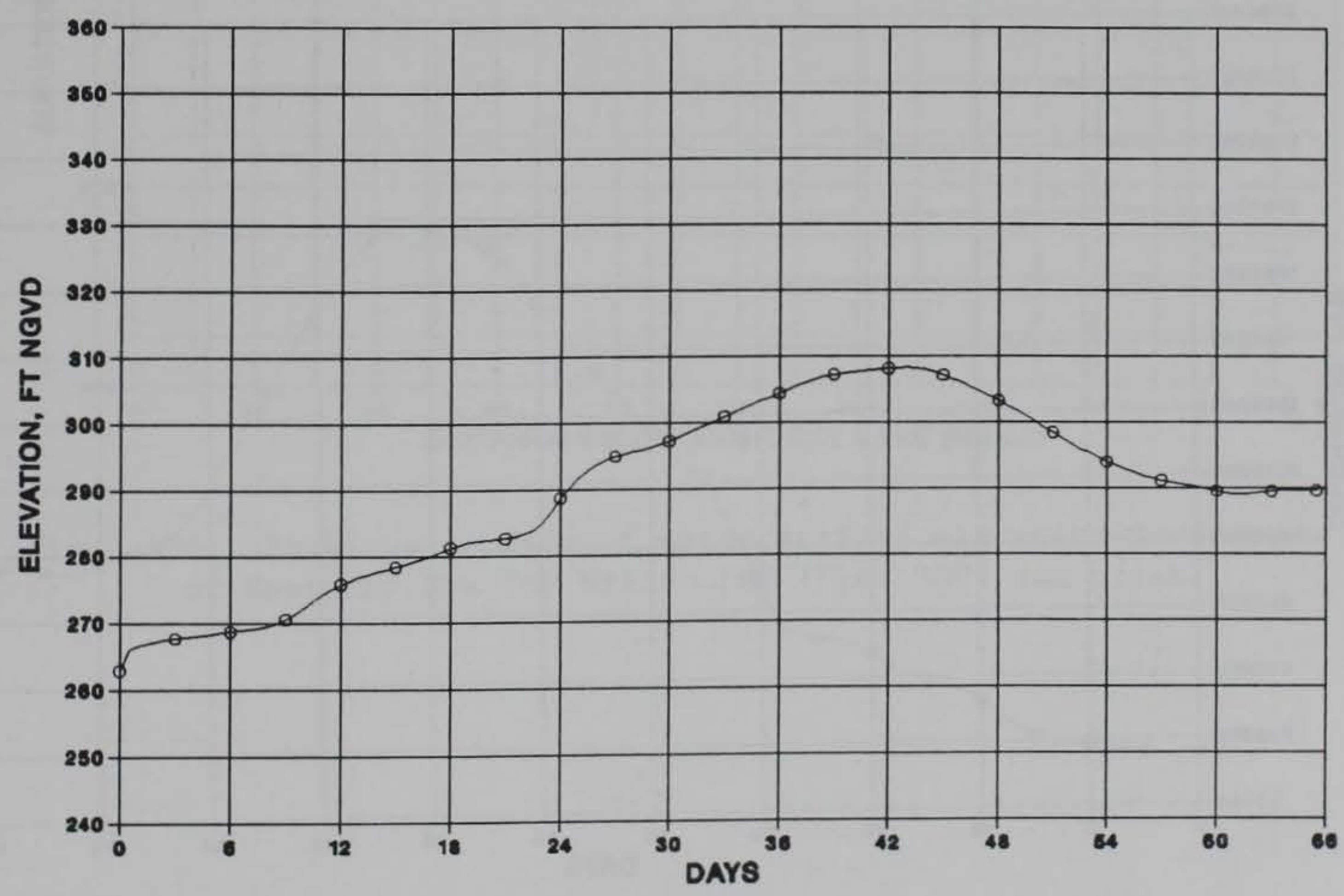

b. Elevation

Figure 34. Results at New Madrid, MO, for Wilson PMF (Elev 380) dam break 


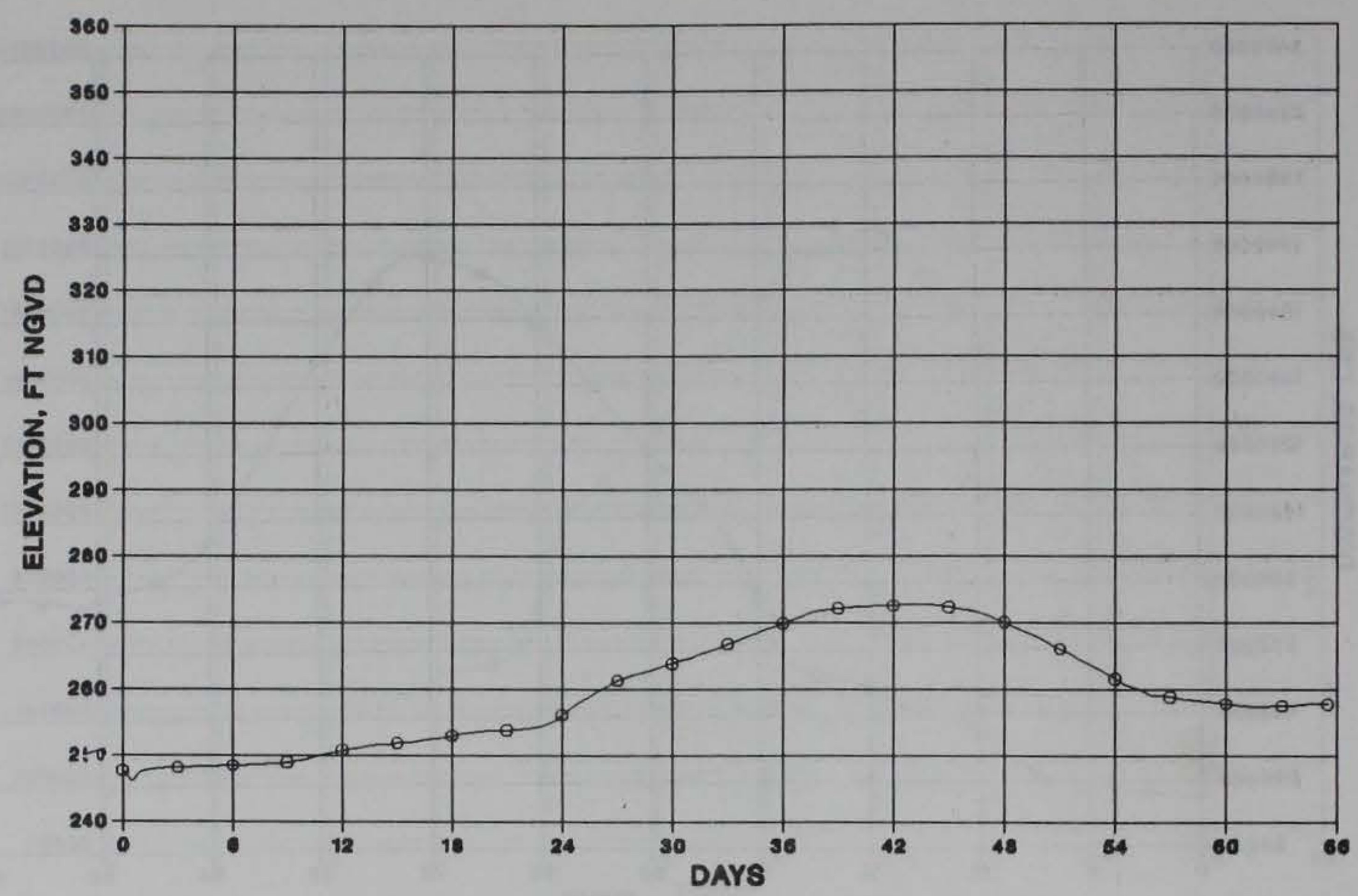

a. Discharge

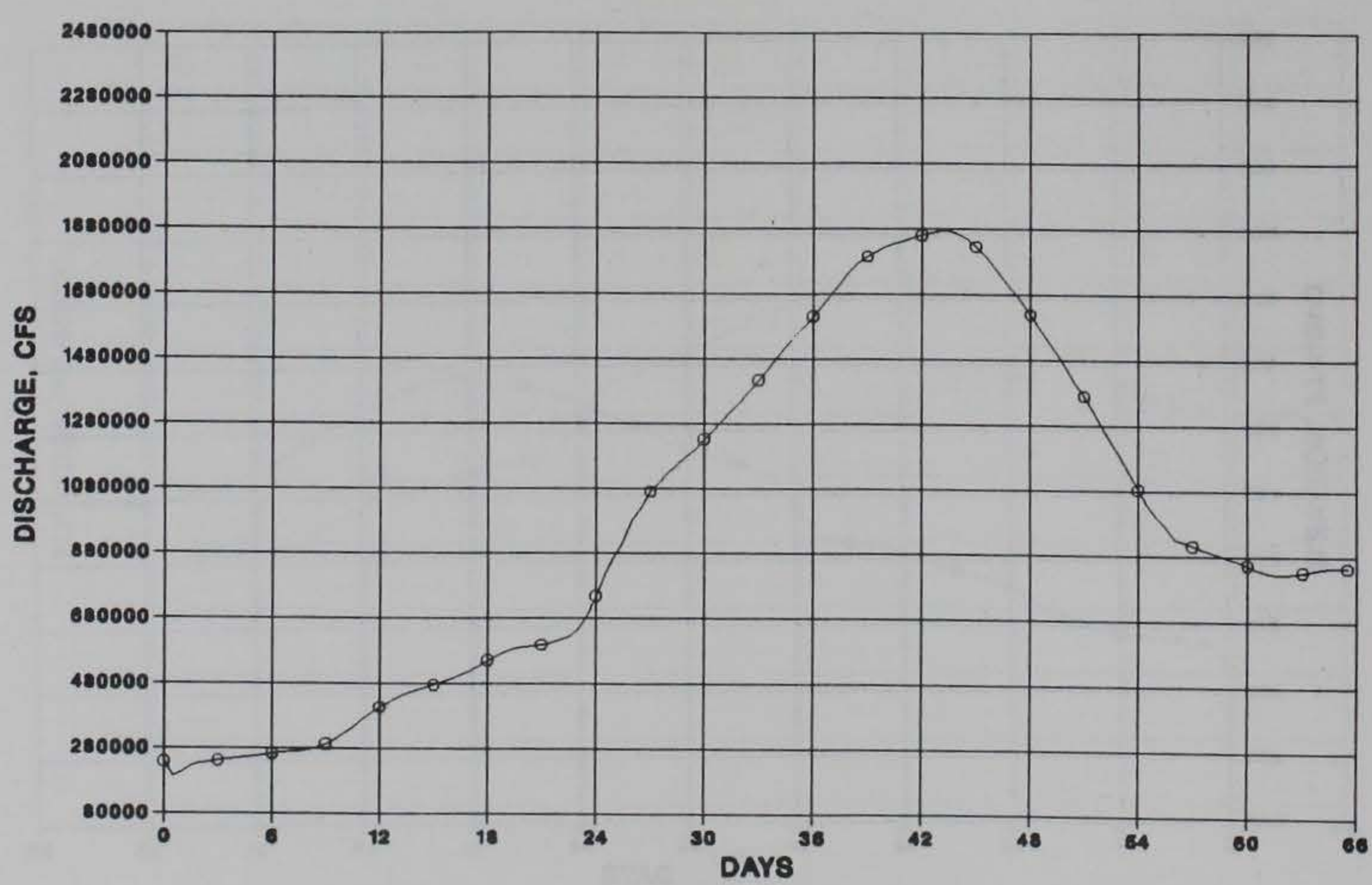

b. Elevation

Figure 35. Results at Caruthersville, MO, for Wilson PMF (Elev 380) dam break 


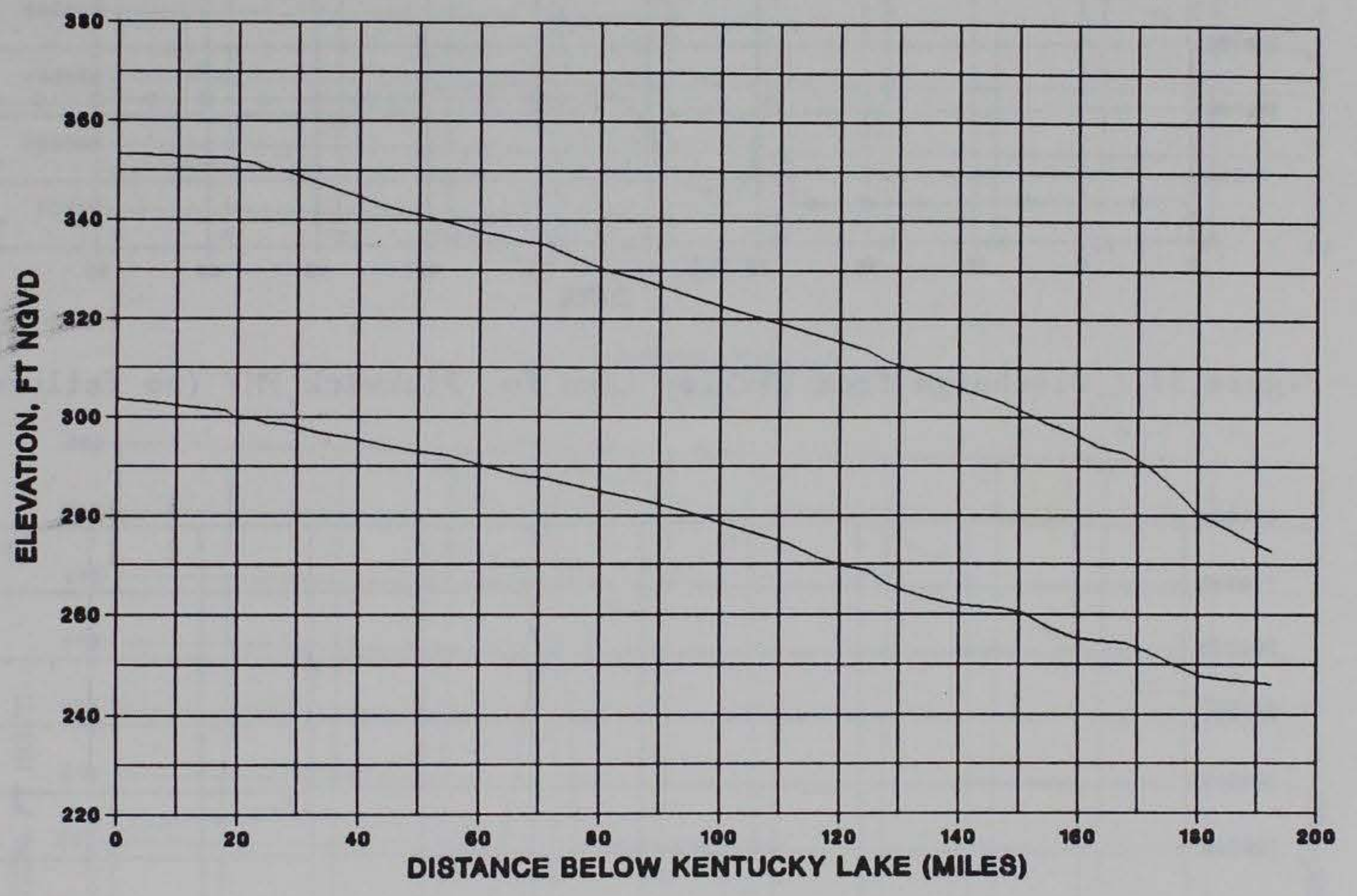

Figure 36. Envelope profile of water surface elevation downstream of Kentucky Dam for Wilson PMF (Elev 380) dam break 


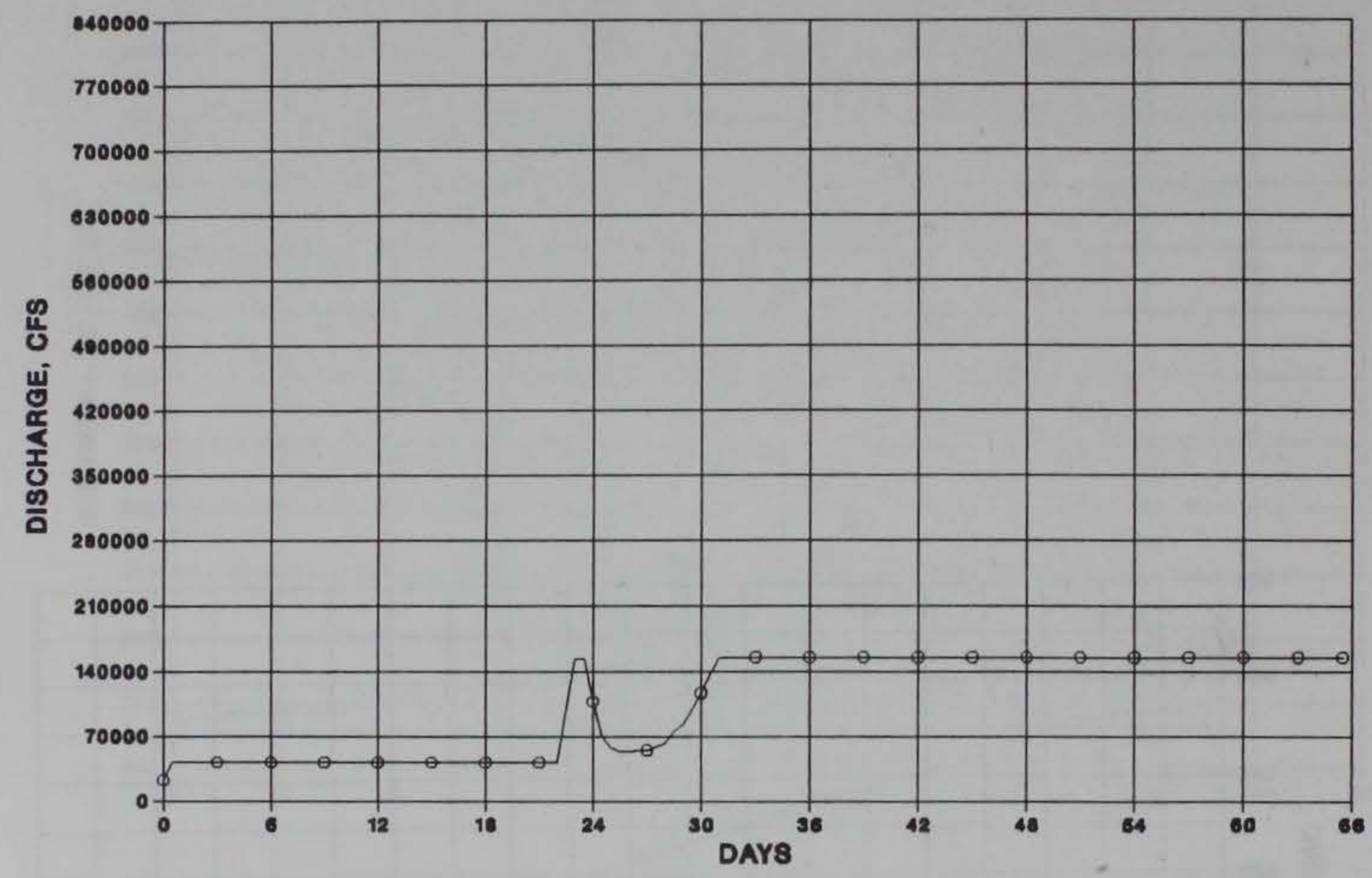

Figure 37. Discharge from Barkley Lake for Pickwick PMF (no failure)

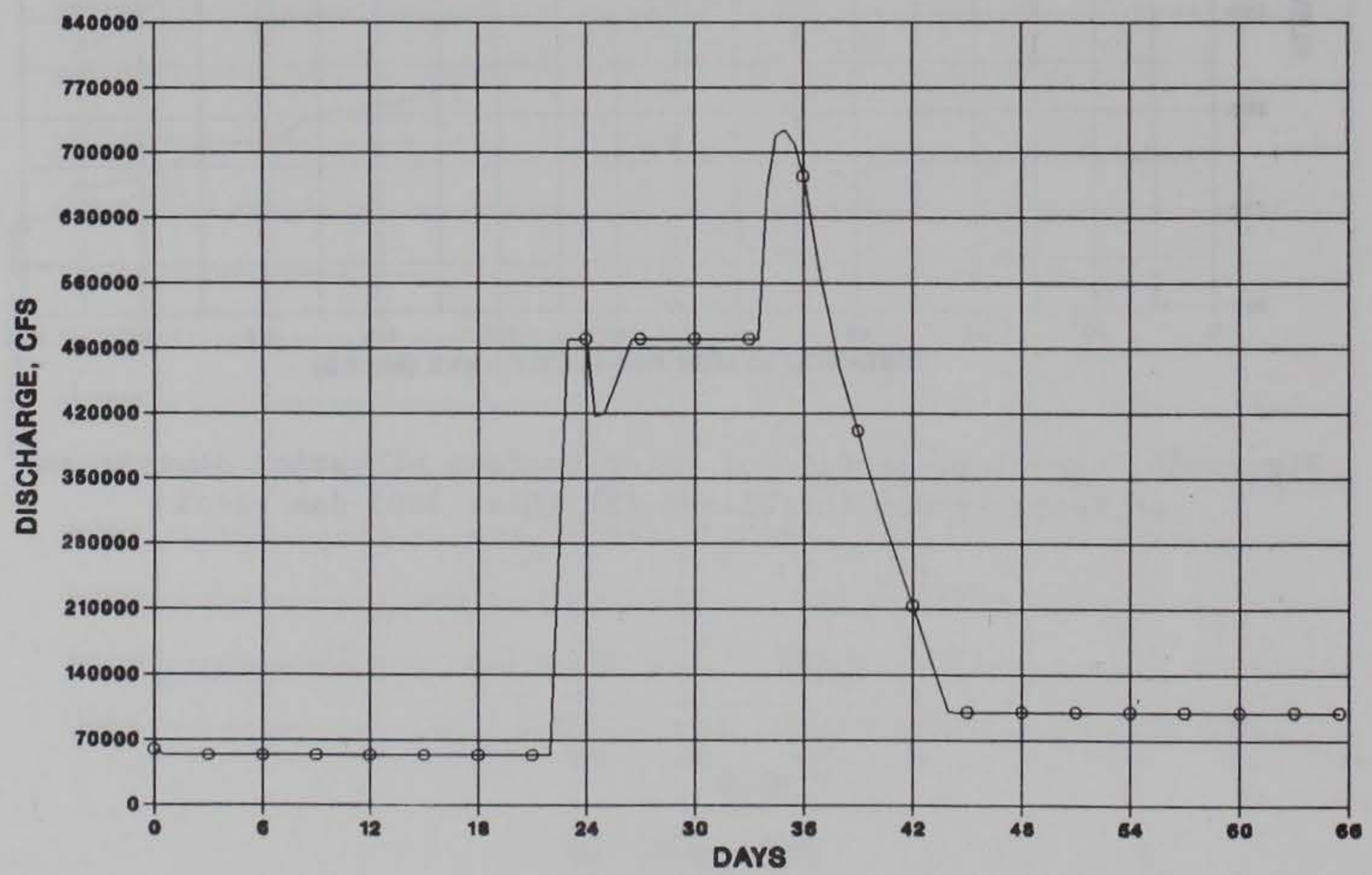

Figure 38. Discharge from Kentucky Lake for Pickwick PMF (no failure) 


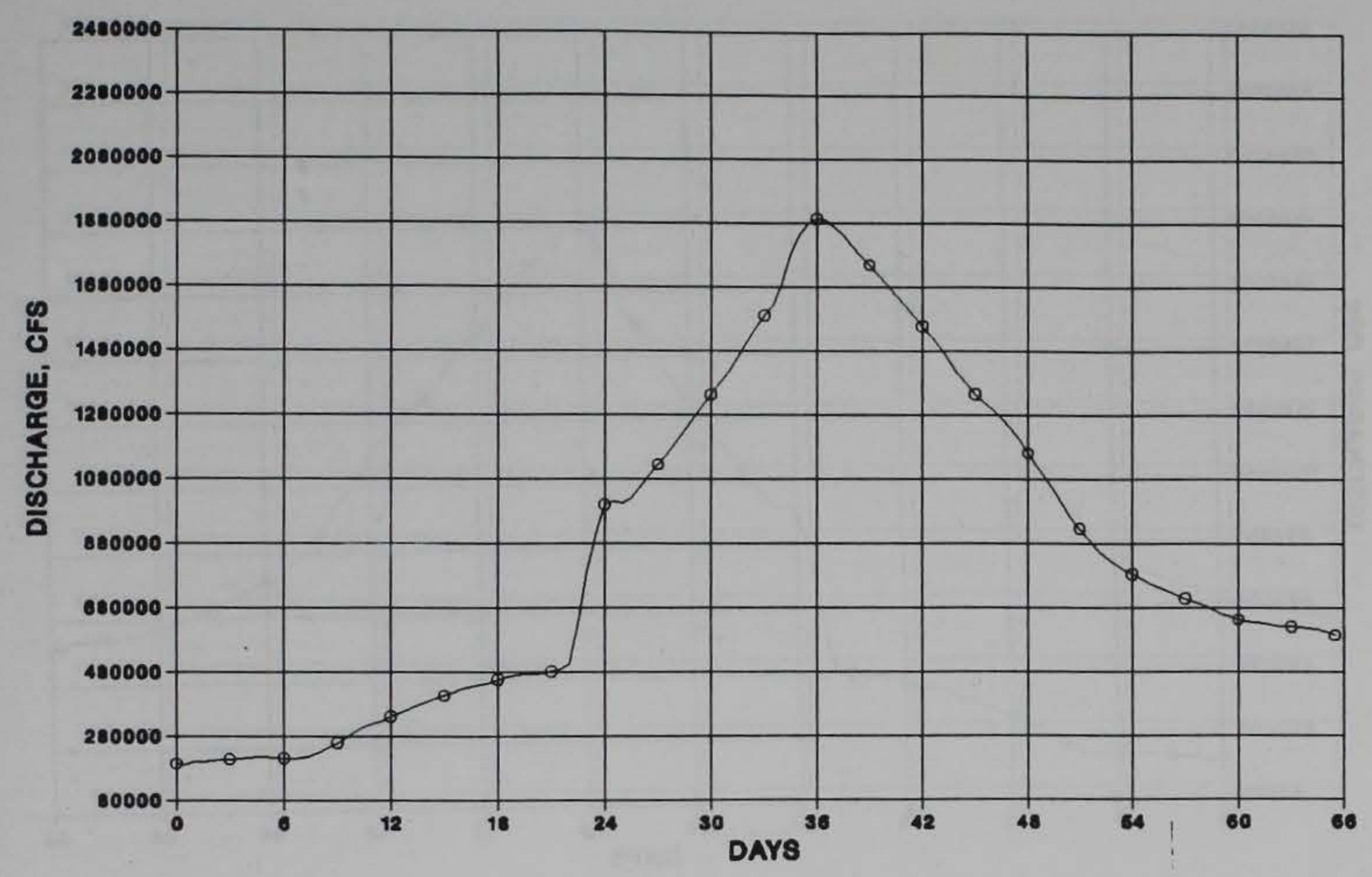

a. Discharge

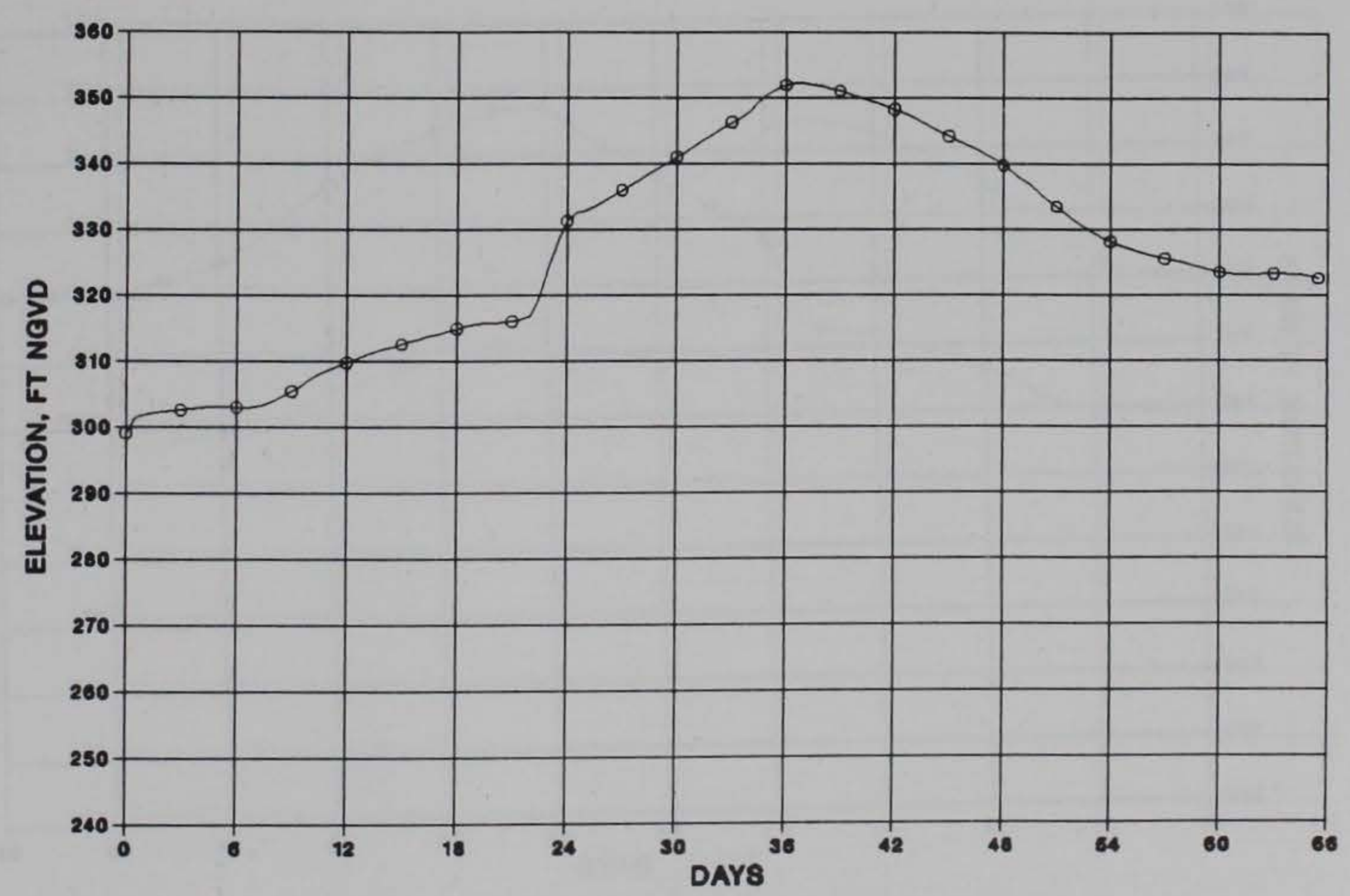

b. Elevation

Figure 39. Results at Paducah, KY, for Pickwick PMF (no failure) 


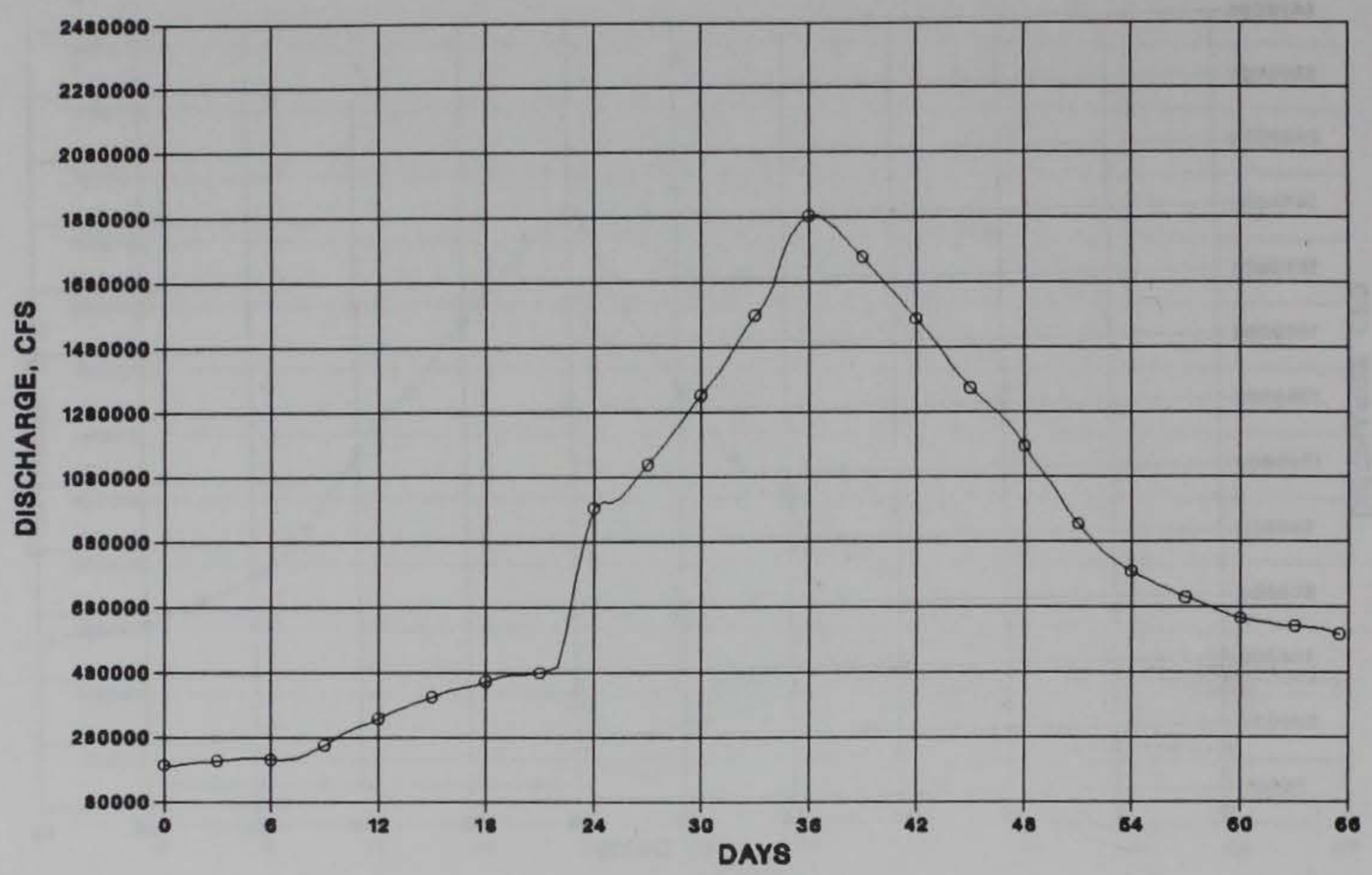

a. Discharge

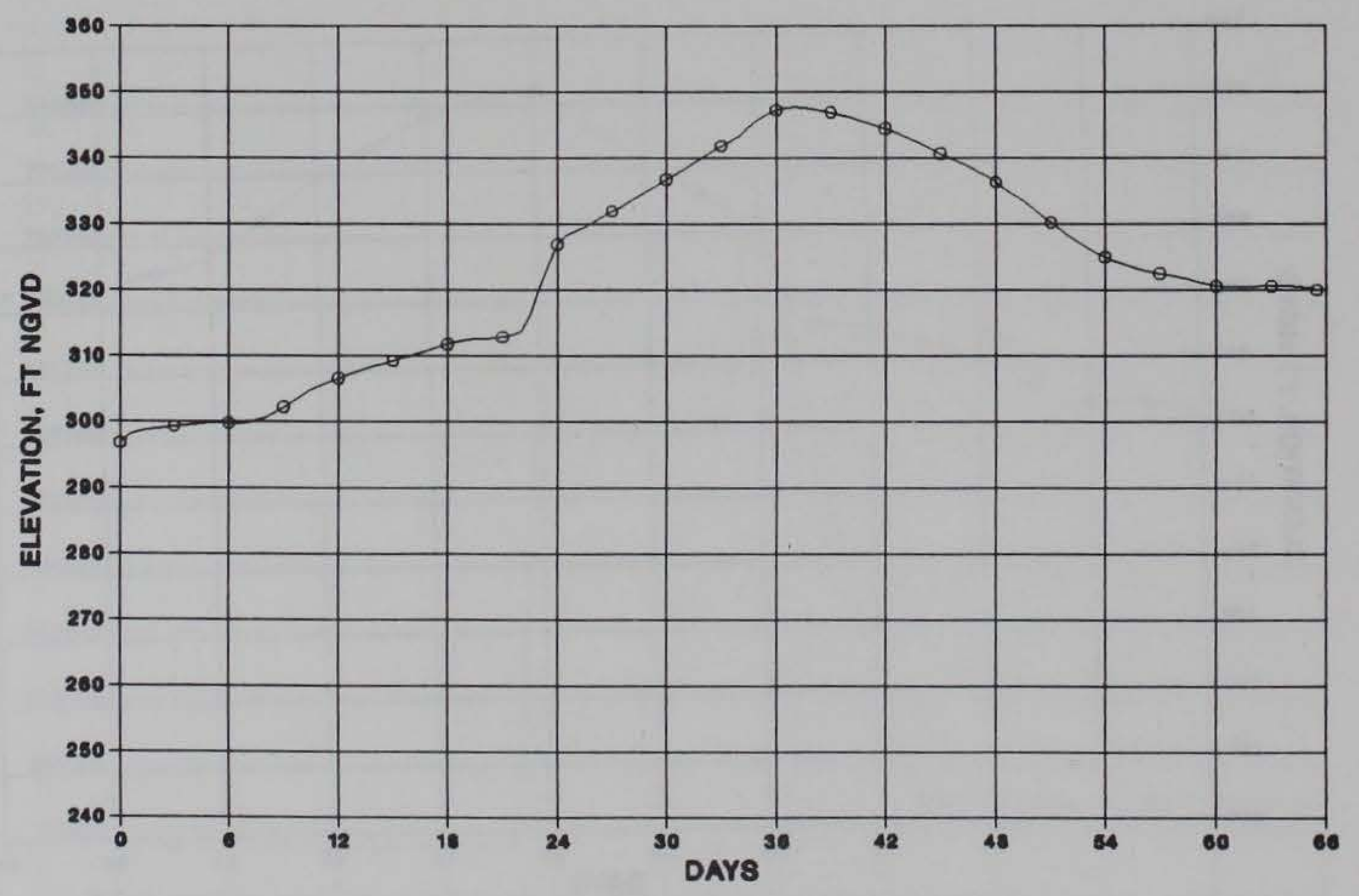

b. Elevation

Figure 40. Results at Metropolis, IL, for Pickwick PMF (no failure) 


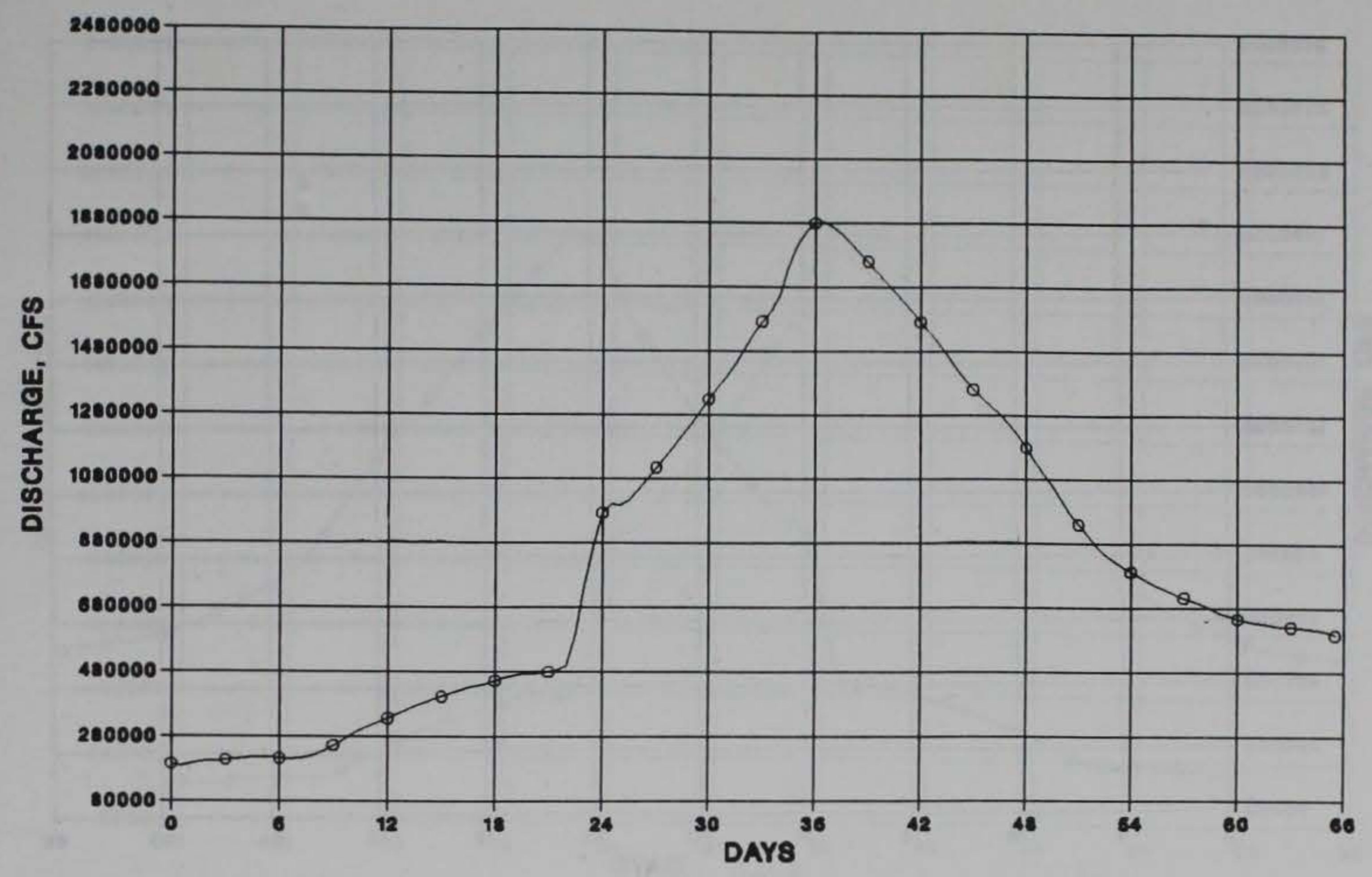

a. Discharge

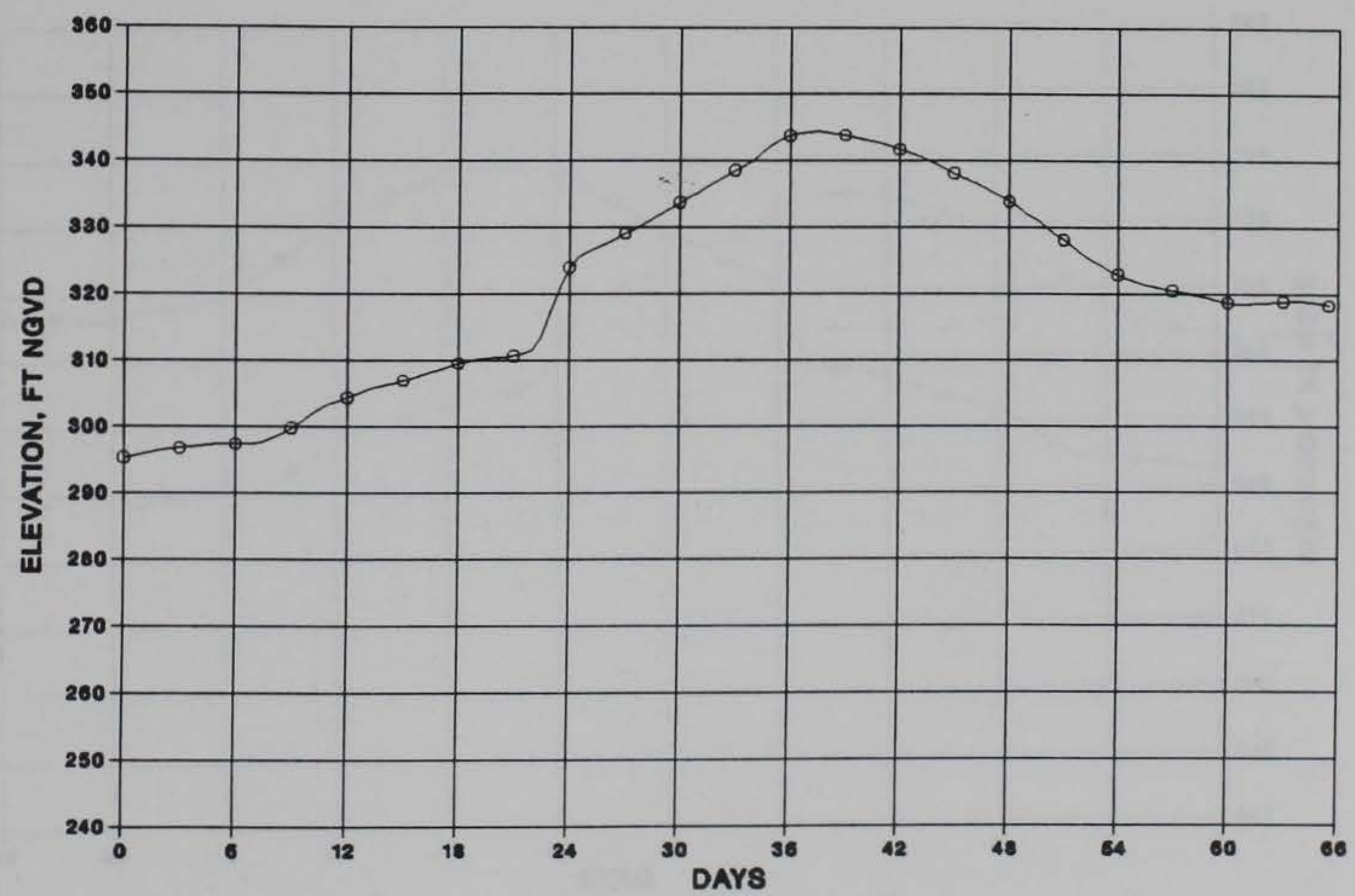

b. Elevation

Figure 41. Results at Joppa, IL, for Pickwick PMF (no failure) 


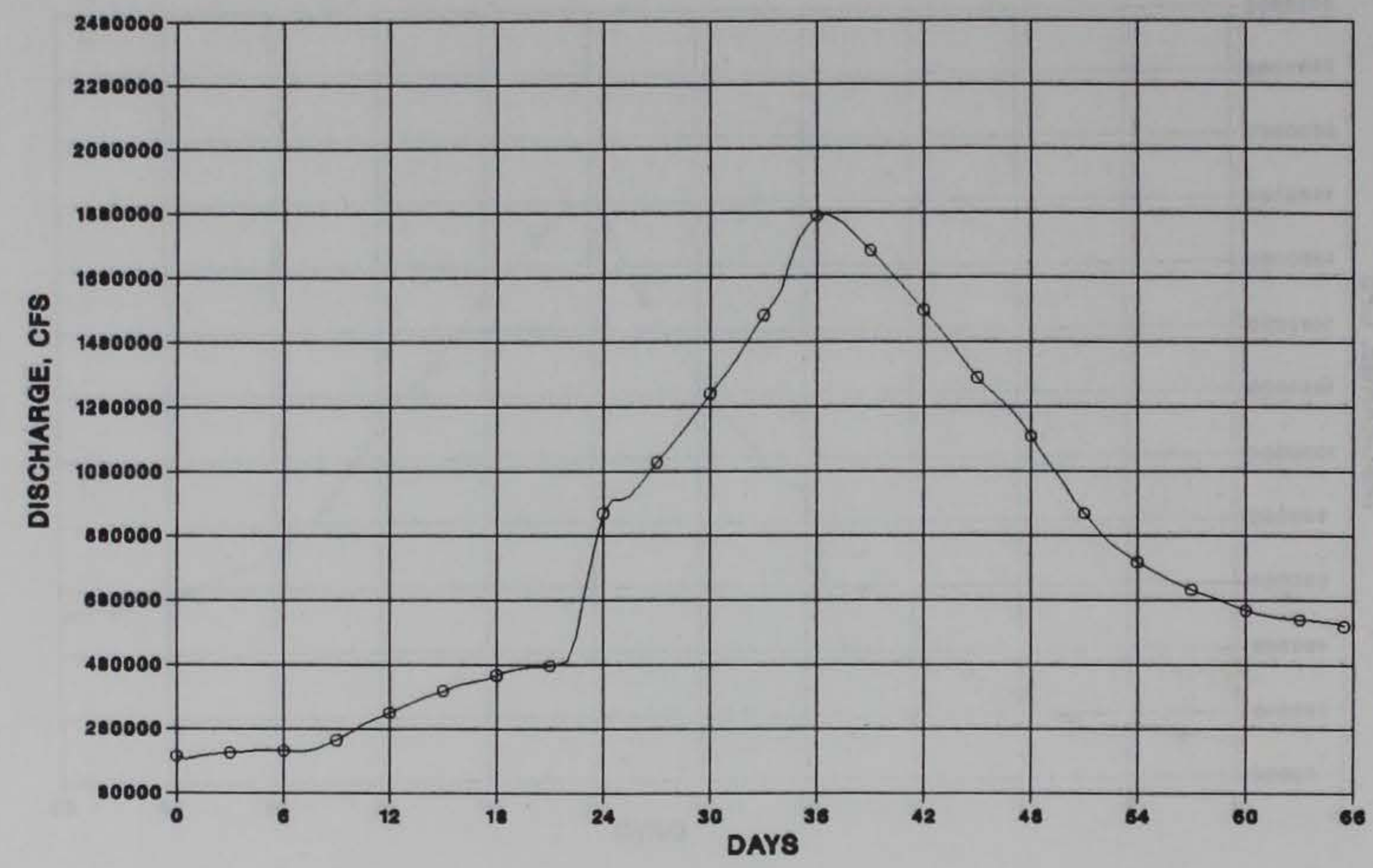

a. Discharge

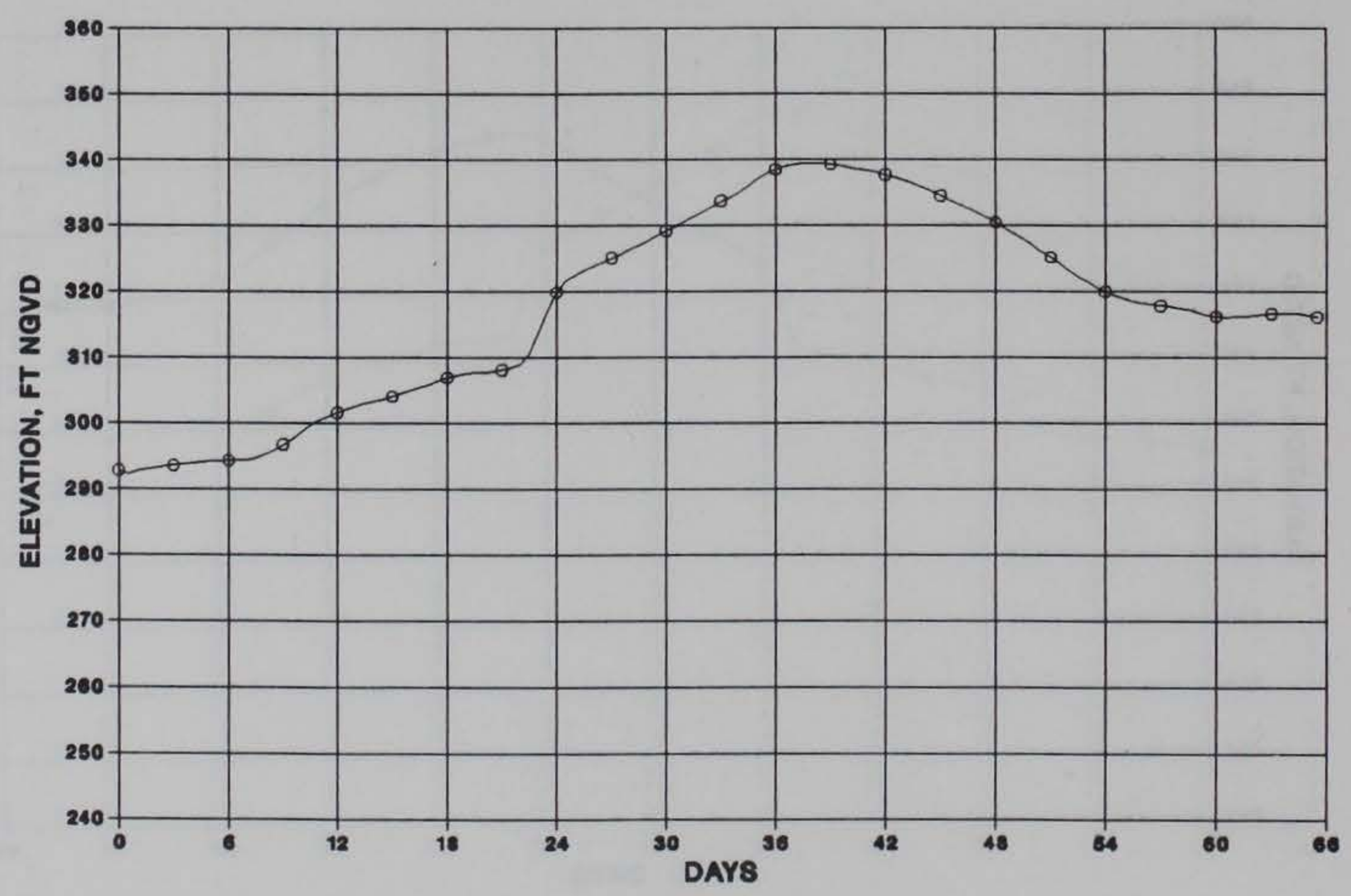

b. Elevation

Figure 42. Results at 0lmstead, IL, for Pickwick PMF (no failure) 


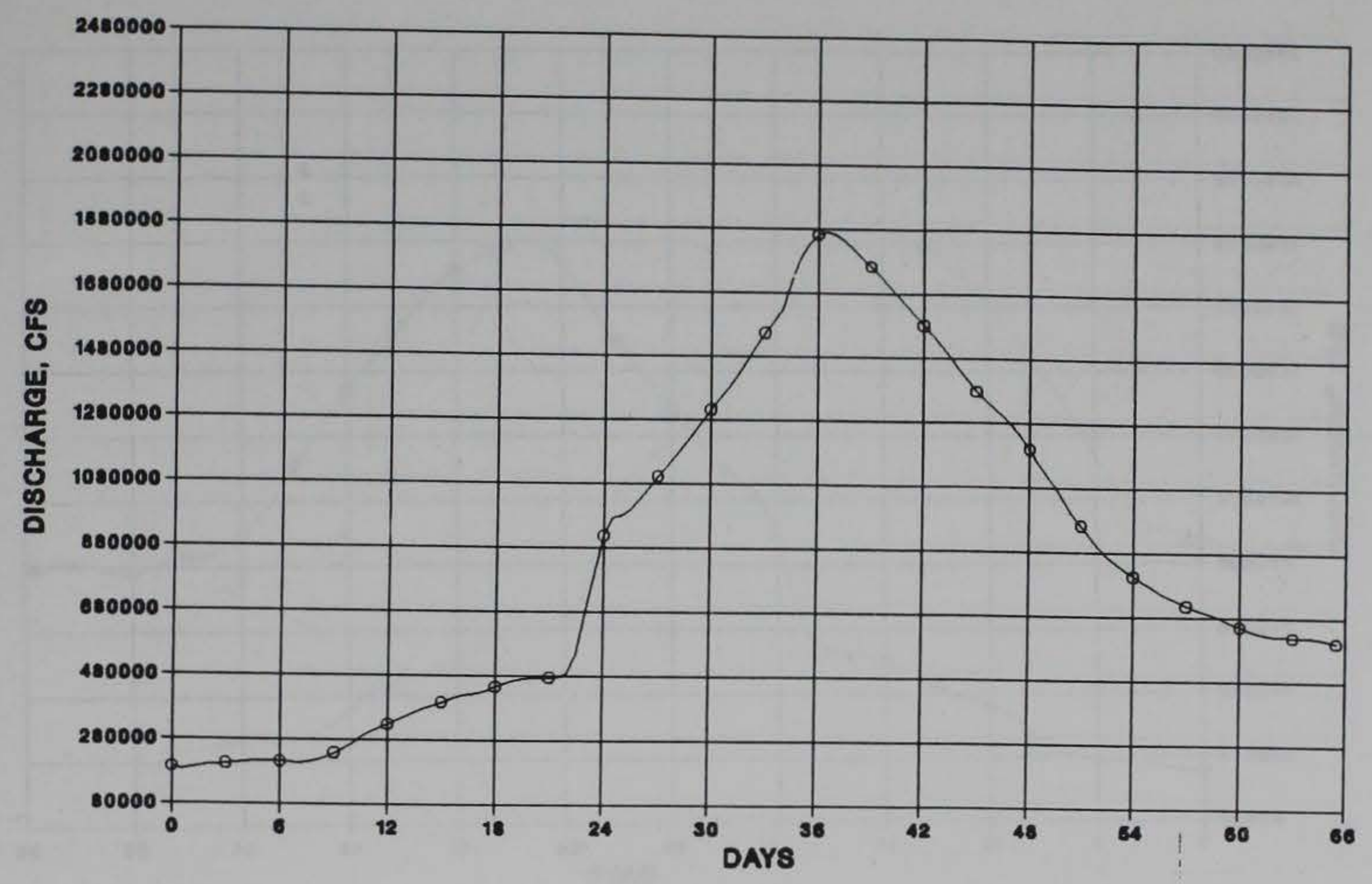

a. Discharge

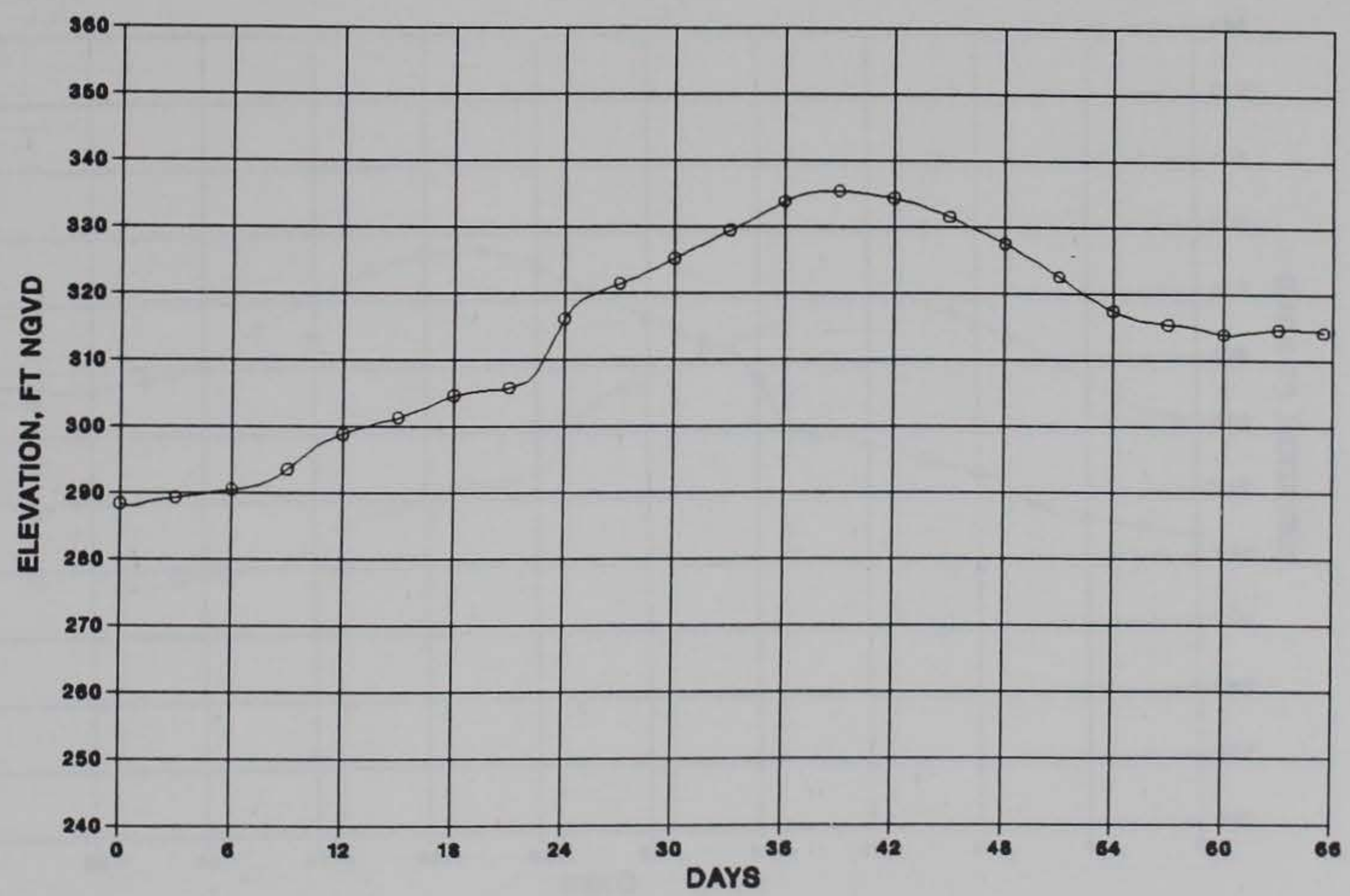

b. Elevation

Figure 43. Results at Cairo, IL, for Pickwick PMF (no failure) 


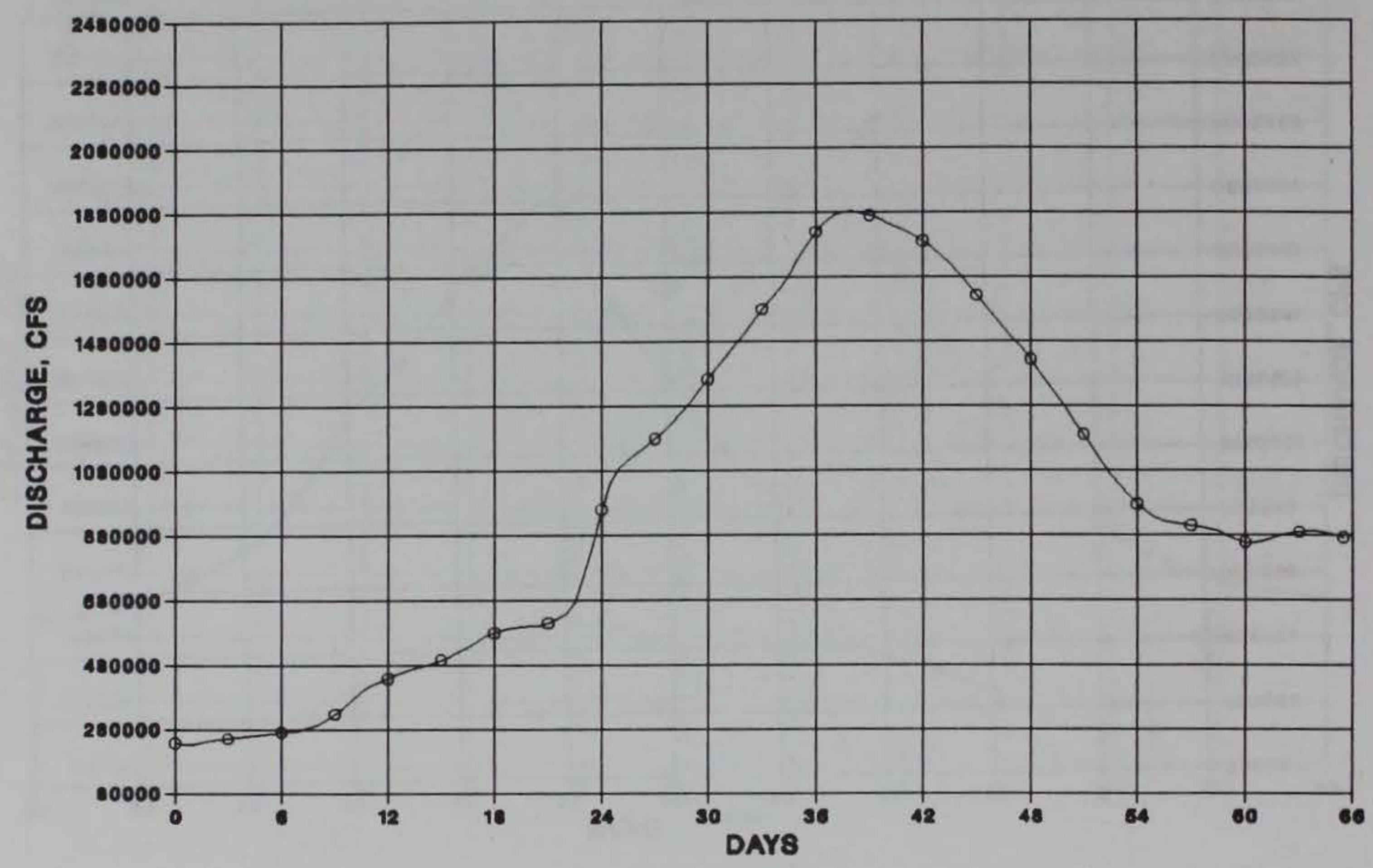

a. Discharge

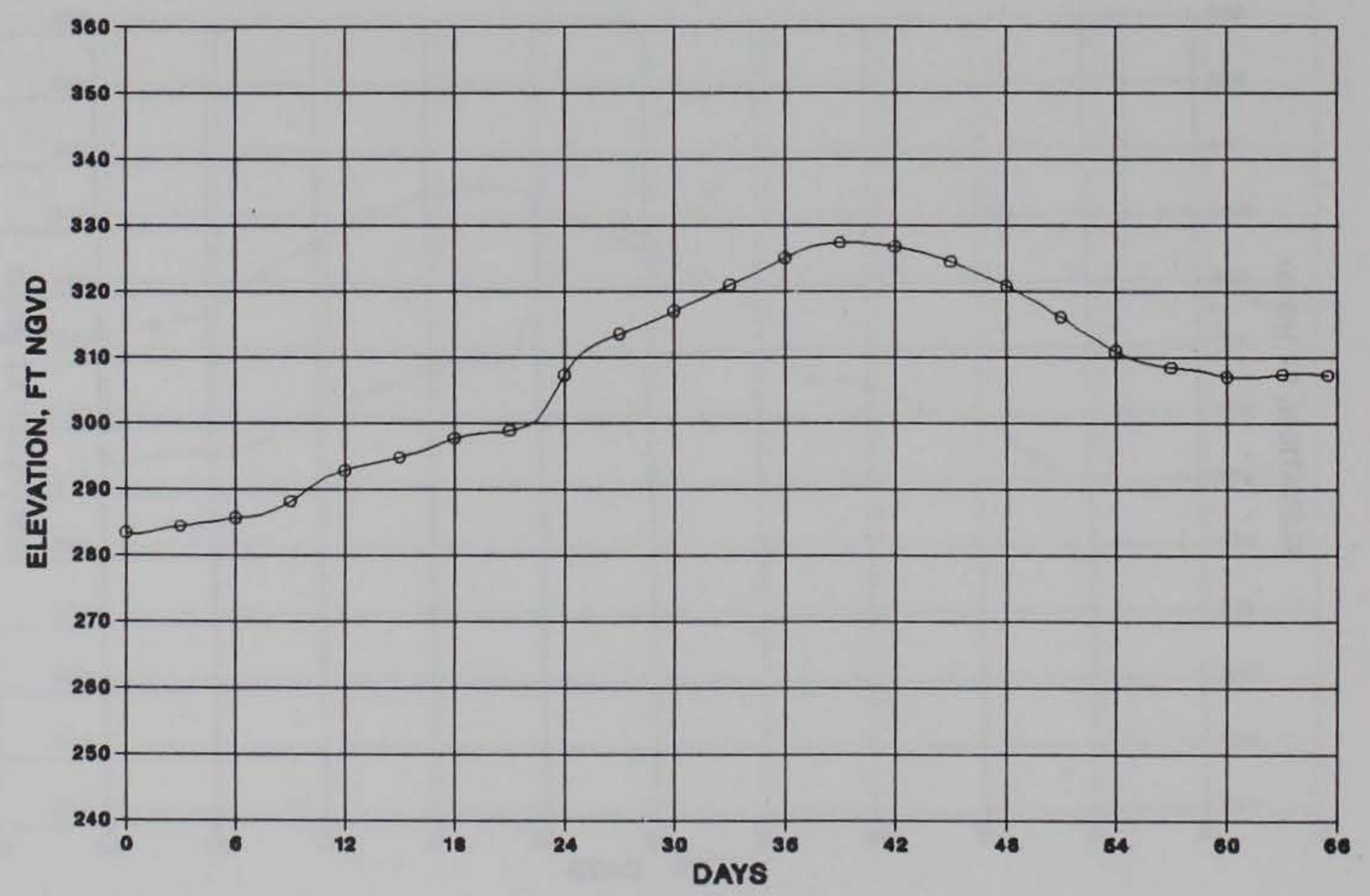

b. Elevation

Figure 44. Results at Columbus, KY, for Pickwick PMF (no failure) 


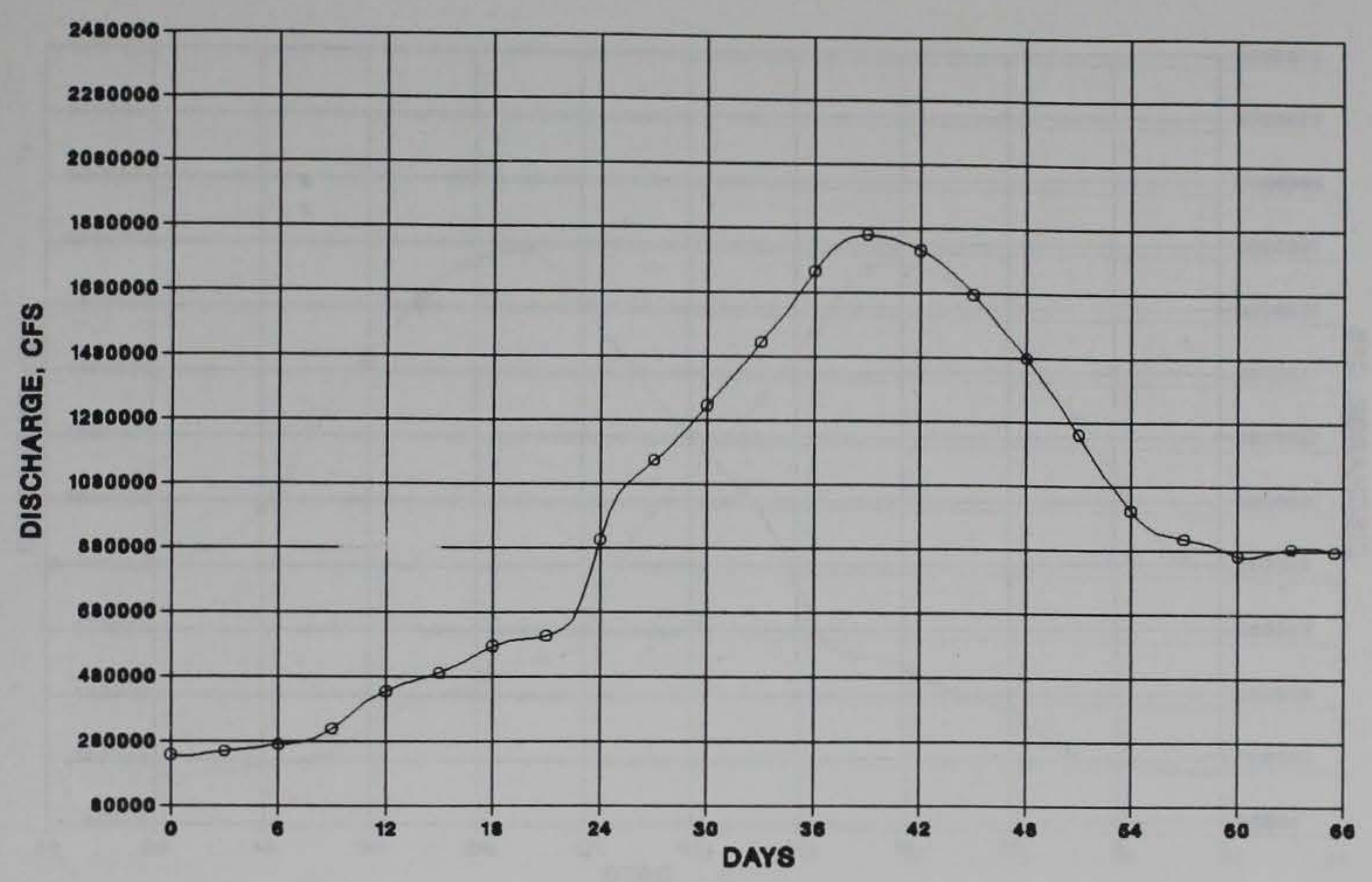

a. Discharge

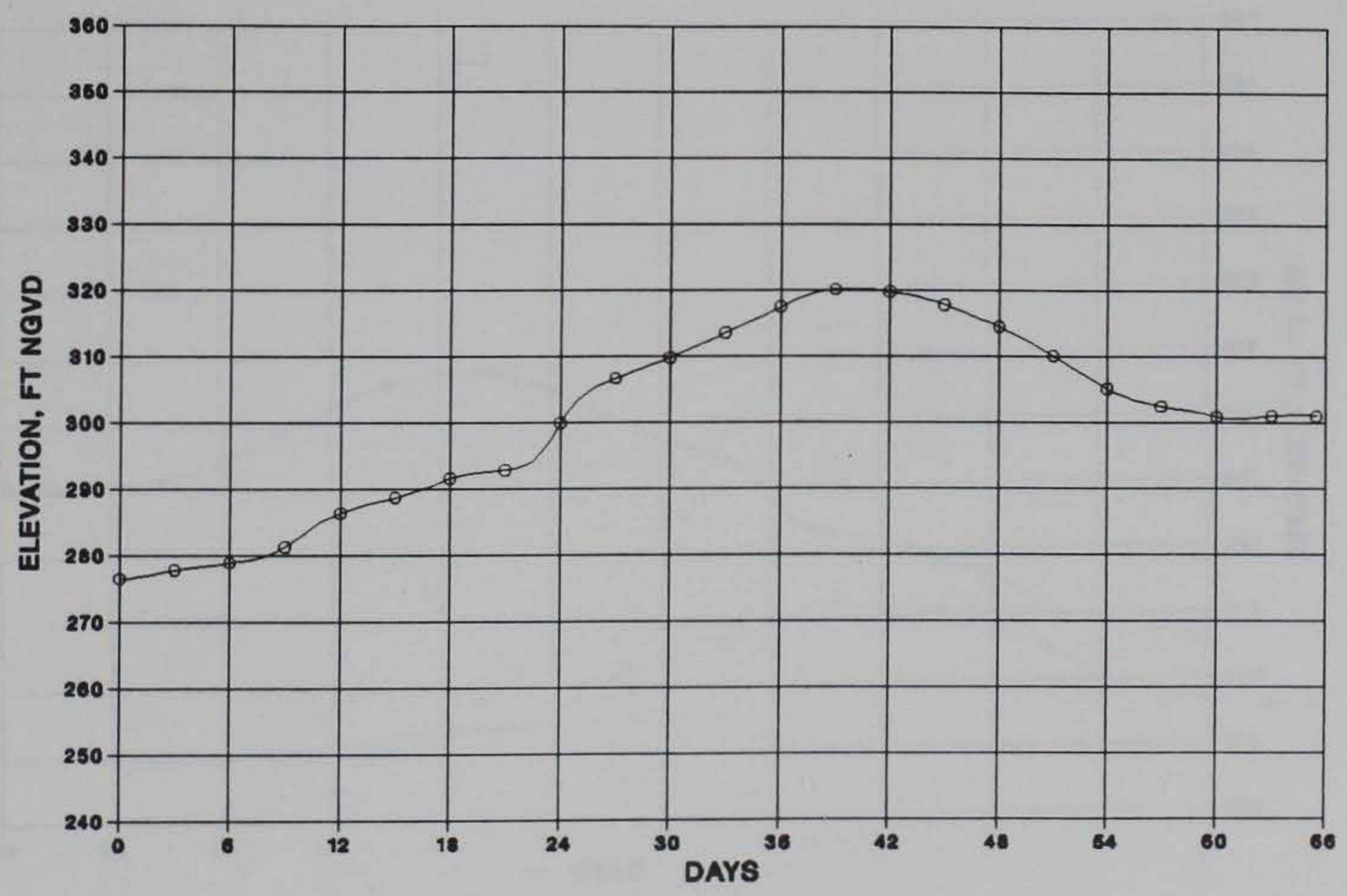

b. Elevation

Figure 45. Results at Hickman, KY, for Pickwick PMF (no failure) 


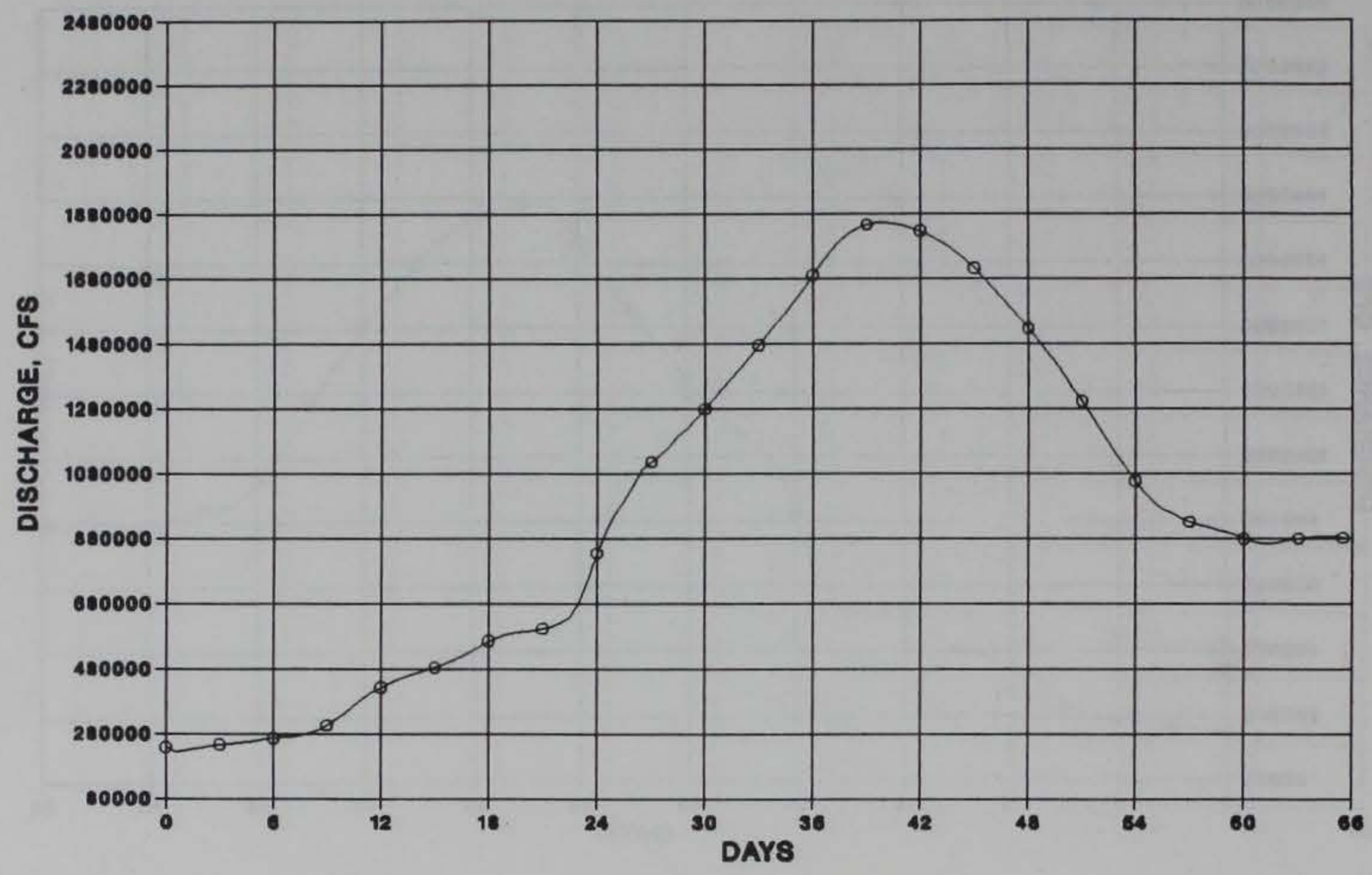

a. Discharge

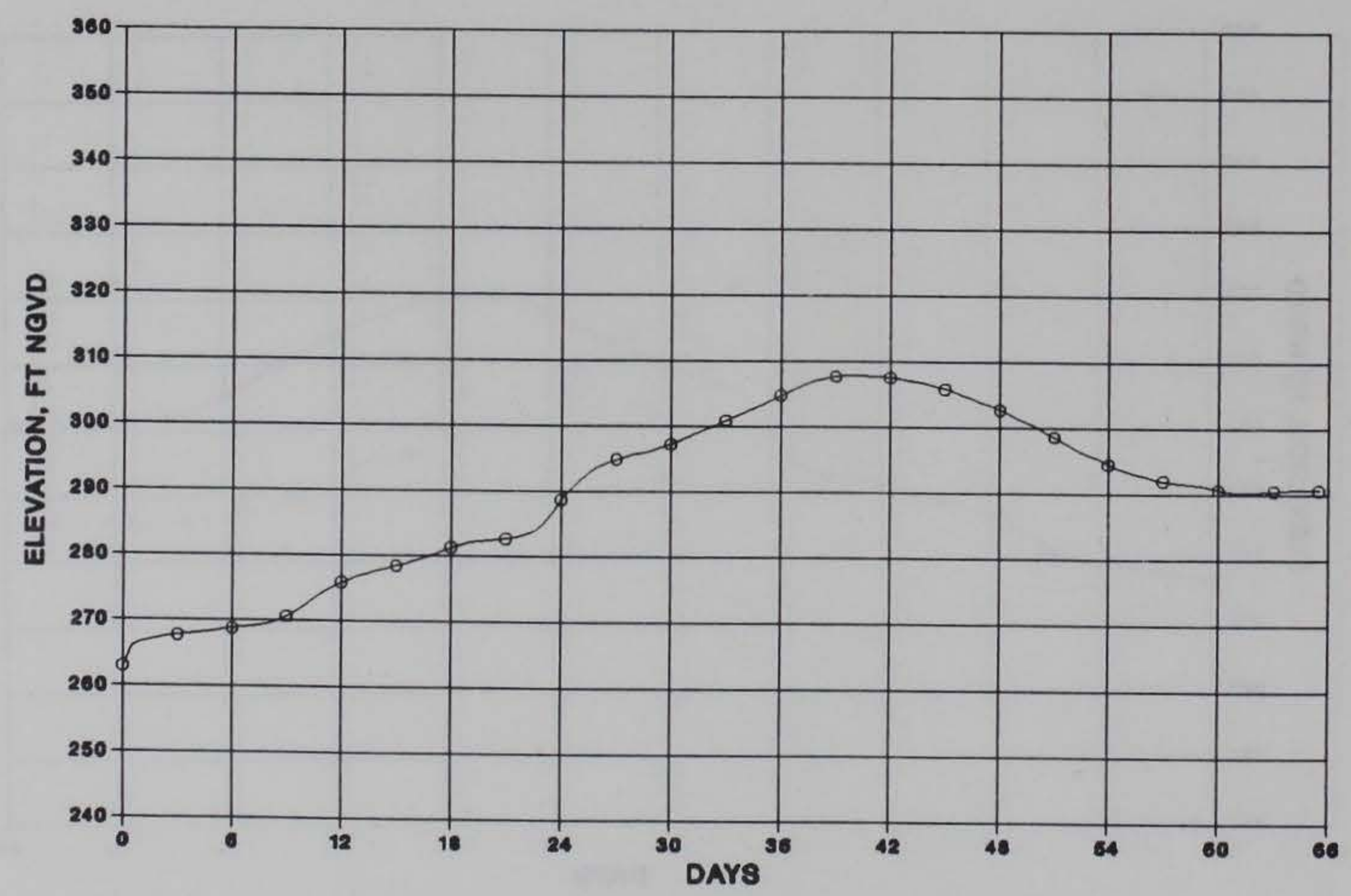

b. Elevation

Figure 46. Results at New Madrid, MO, for Pickwick PMF (no failure) 


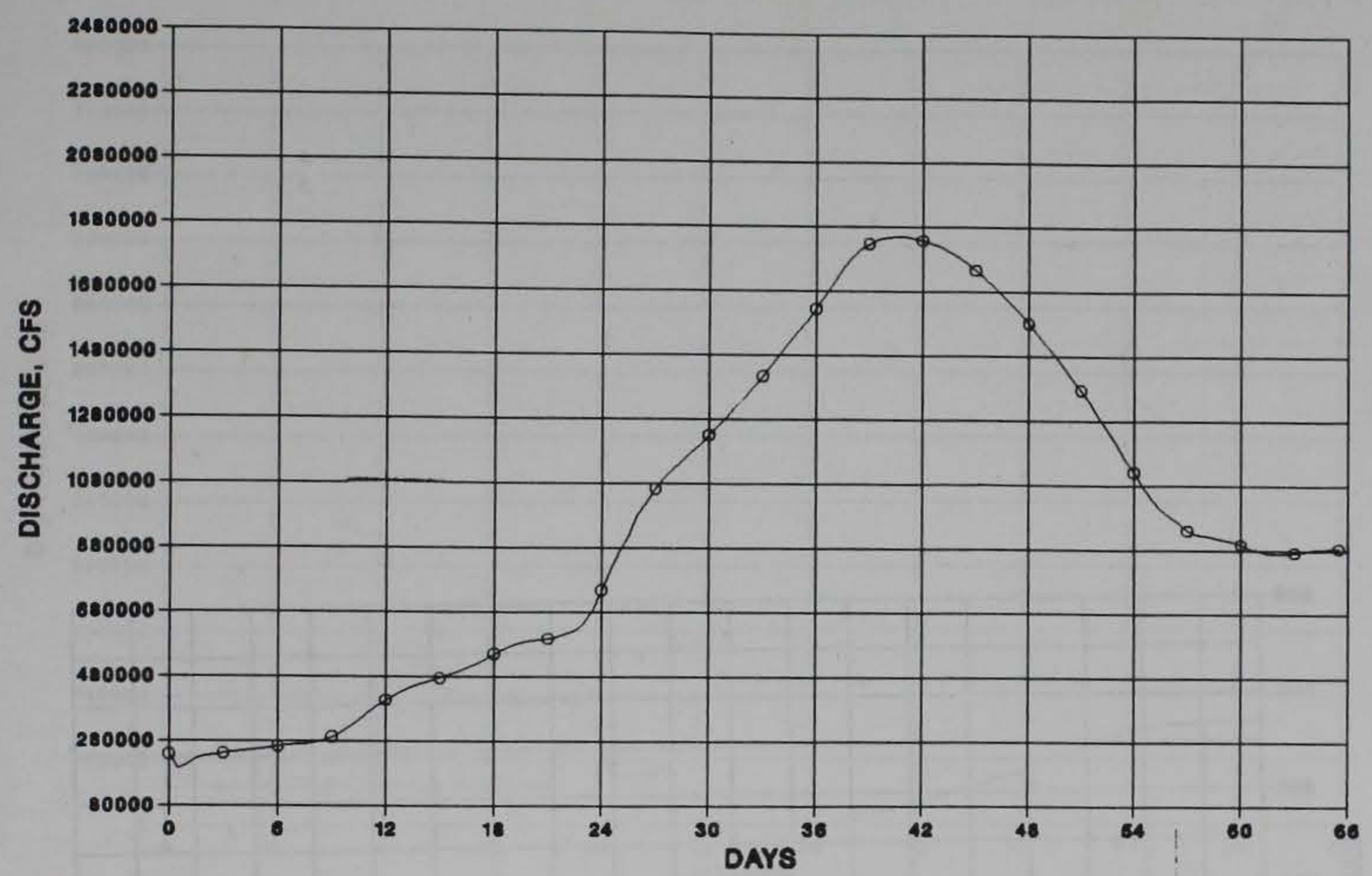

a. Discharge

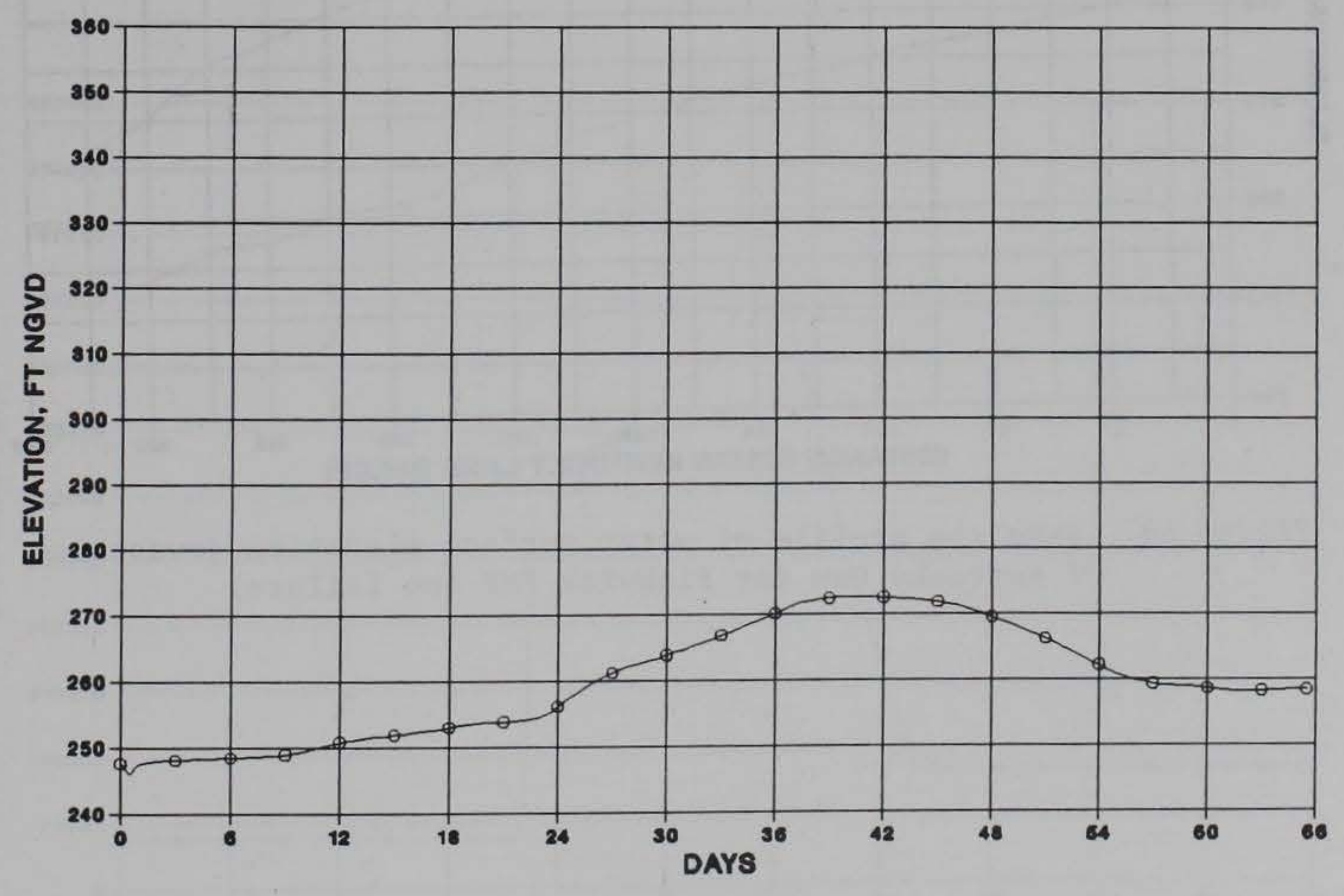

b. Elevation

Figure 47. Results at Caruthersville, MO, for Pickwick PMF (no failure) 


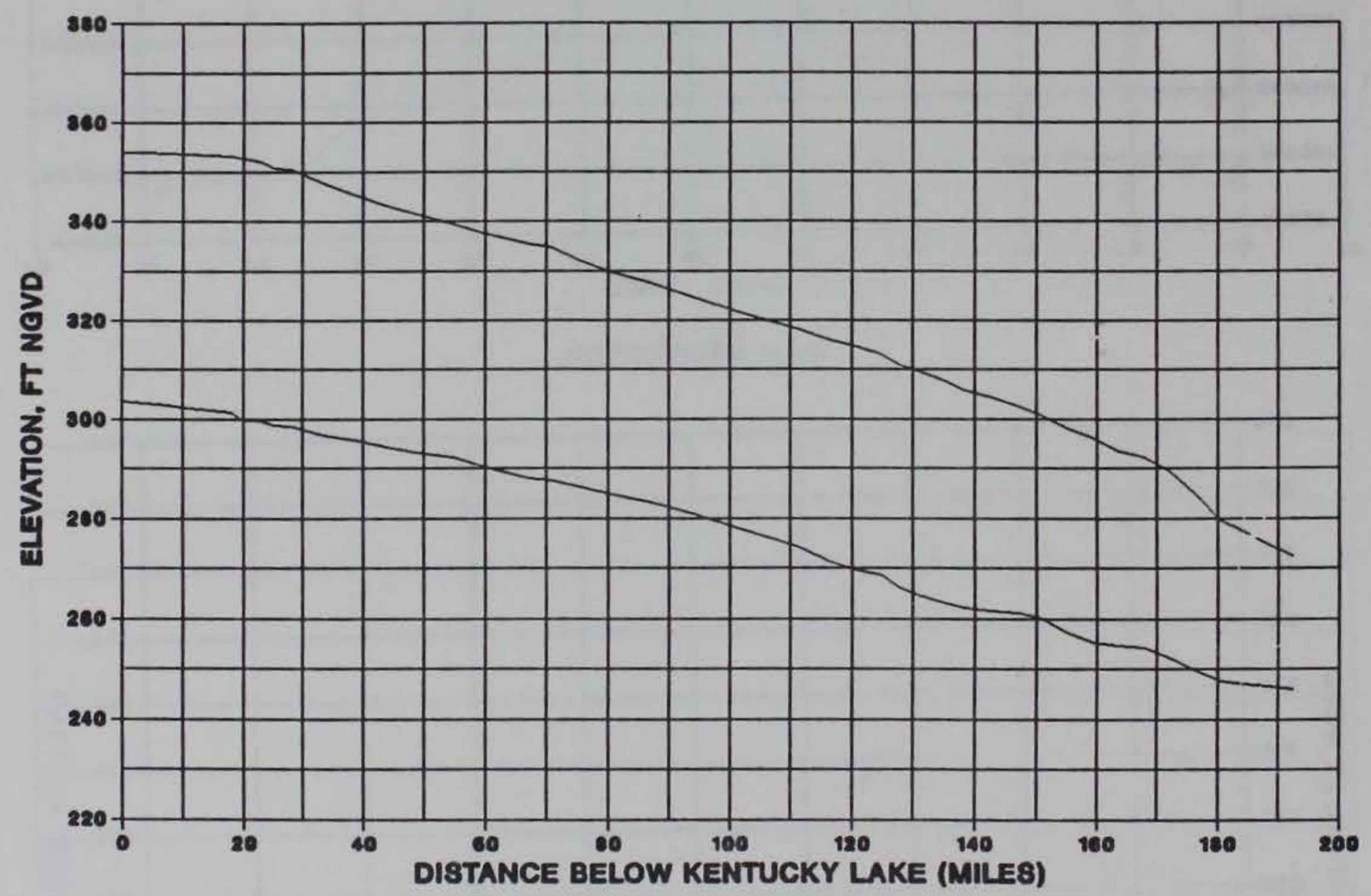

Figure 48. Envelope profile of water surface elevation downstream of Kentucky Dam for Pickwick PMF (no failure) 


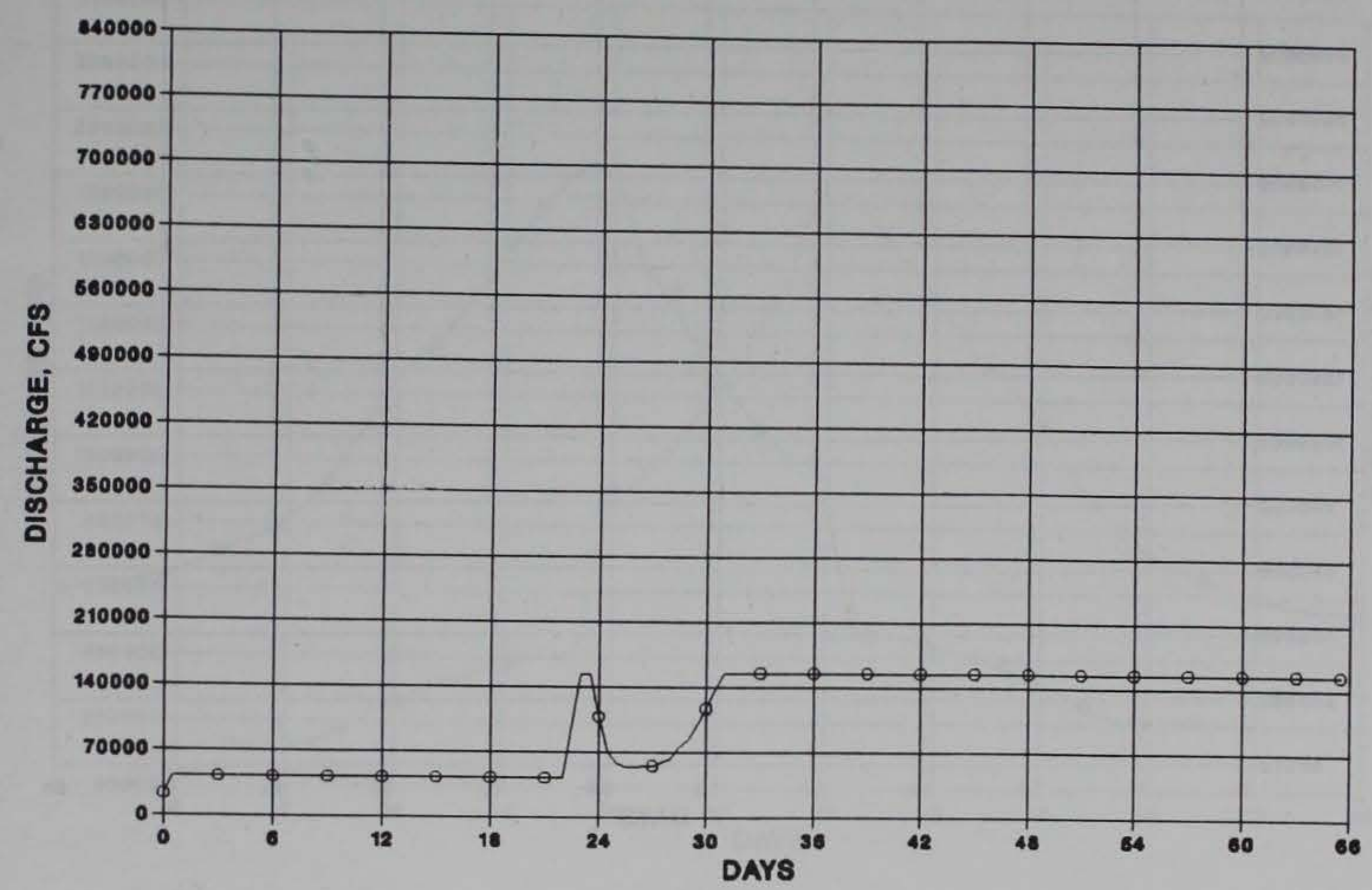

Figure 49. Discharge from Barkley Lake for Pickwick PMF Dam break

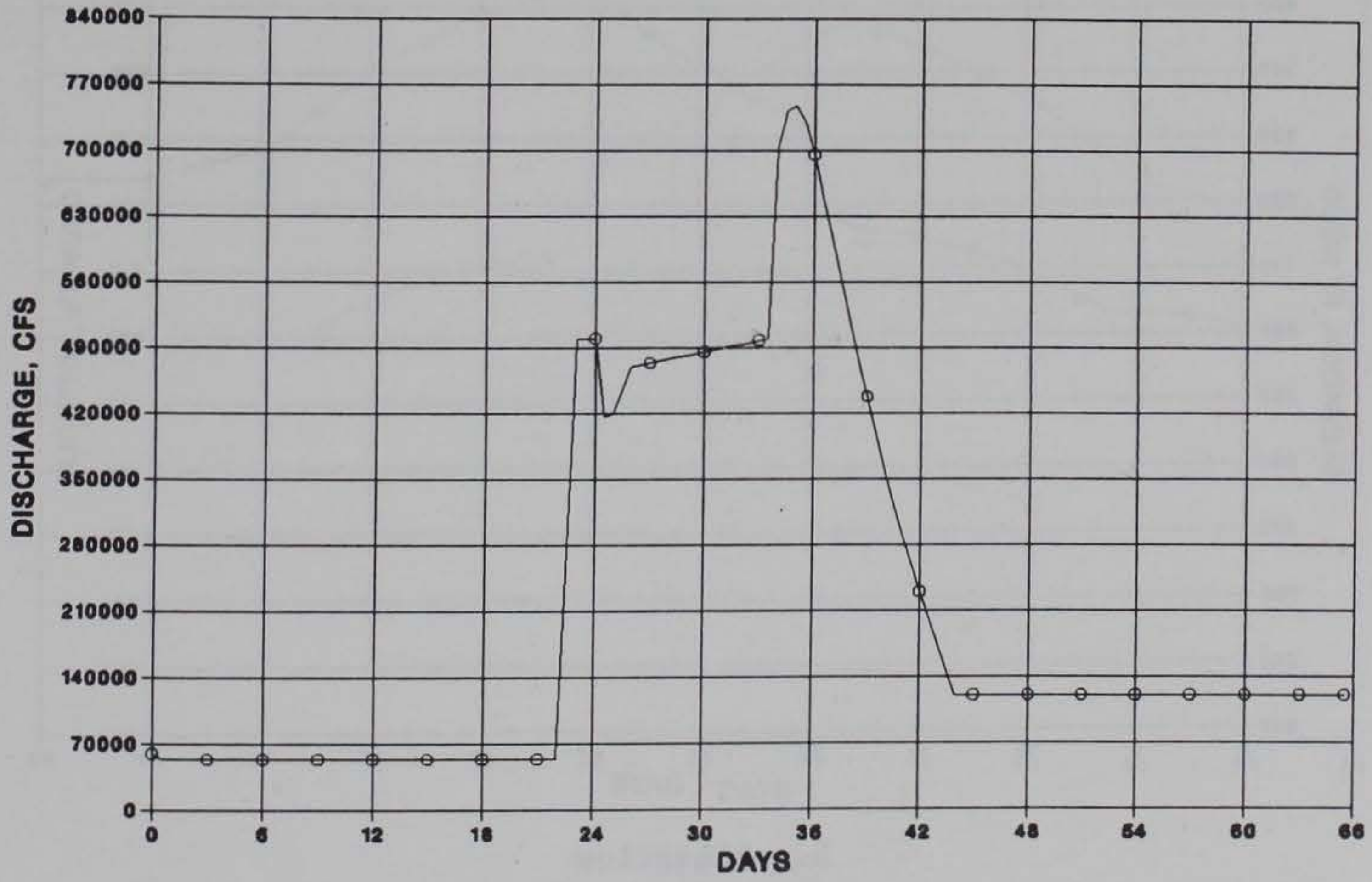

Figure 50. Discharge from Kentucky Lake for Pickwick PMF Dam break 


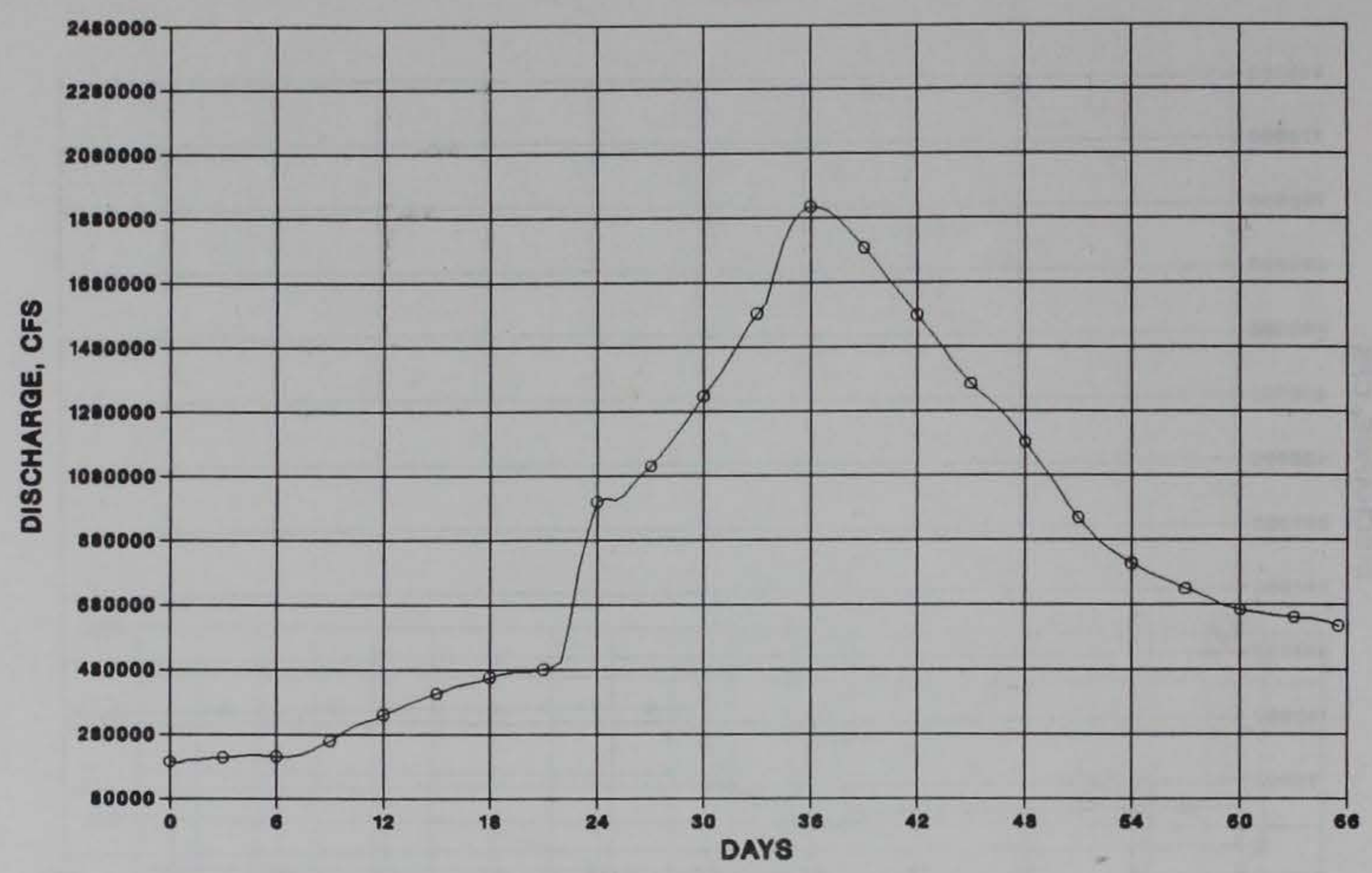

a. Discharge

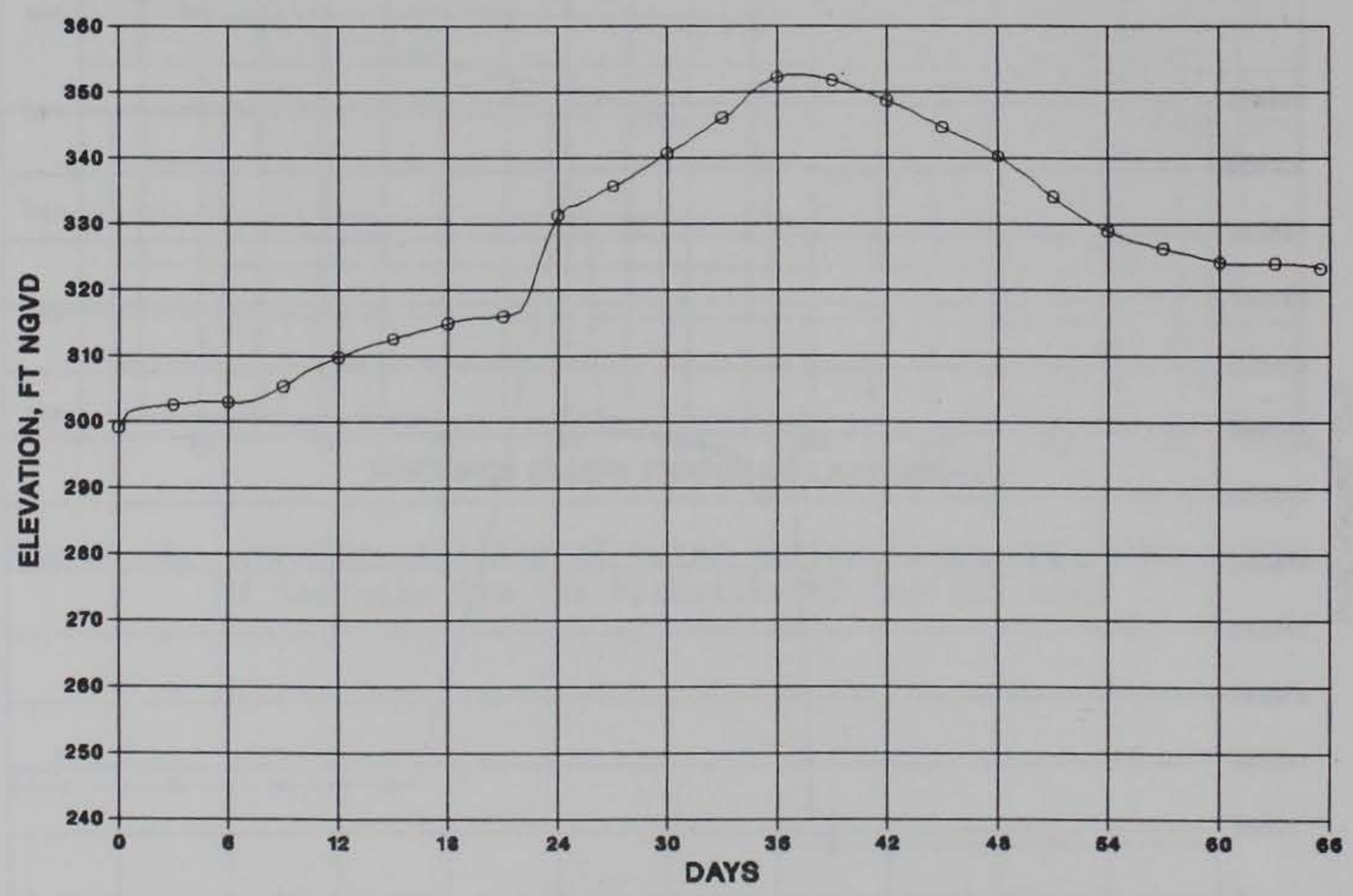

b. Elevation

Figure 51. Results at Paducah, KY, for Pickwick PMF Dam break 


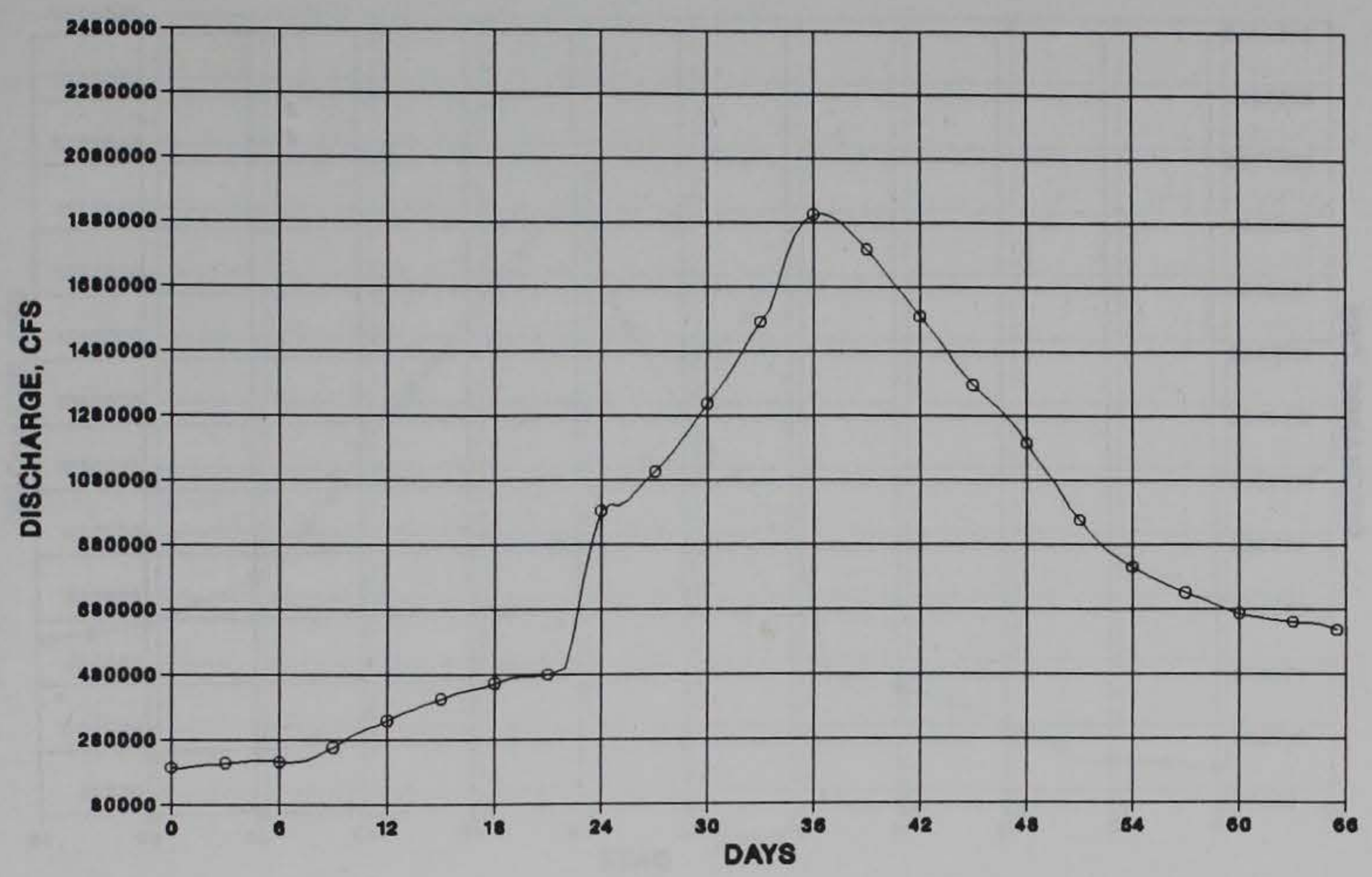

a. Discharge

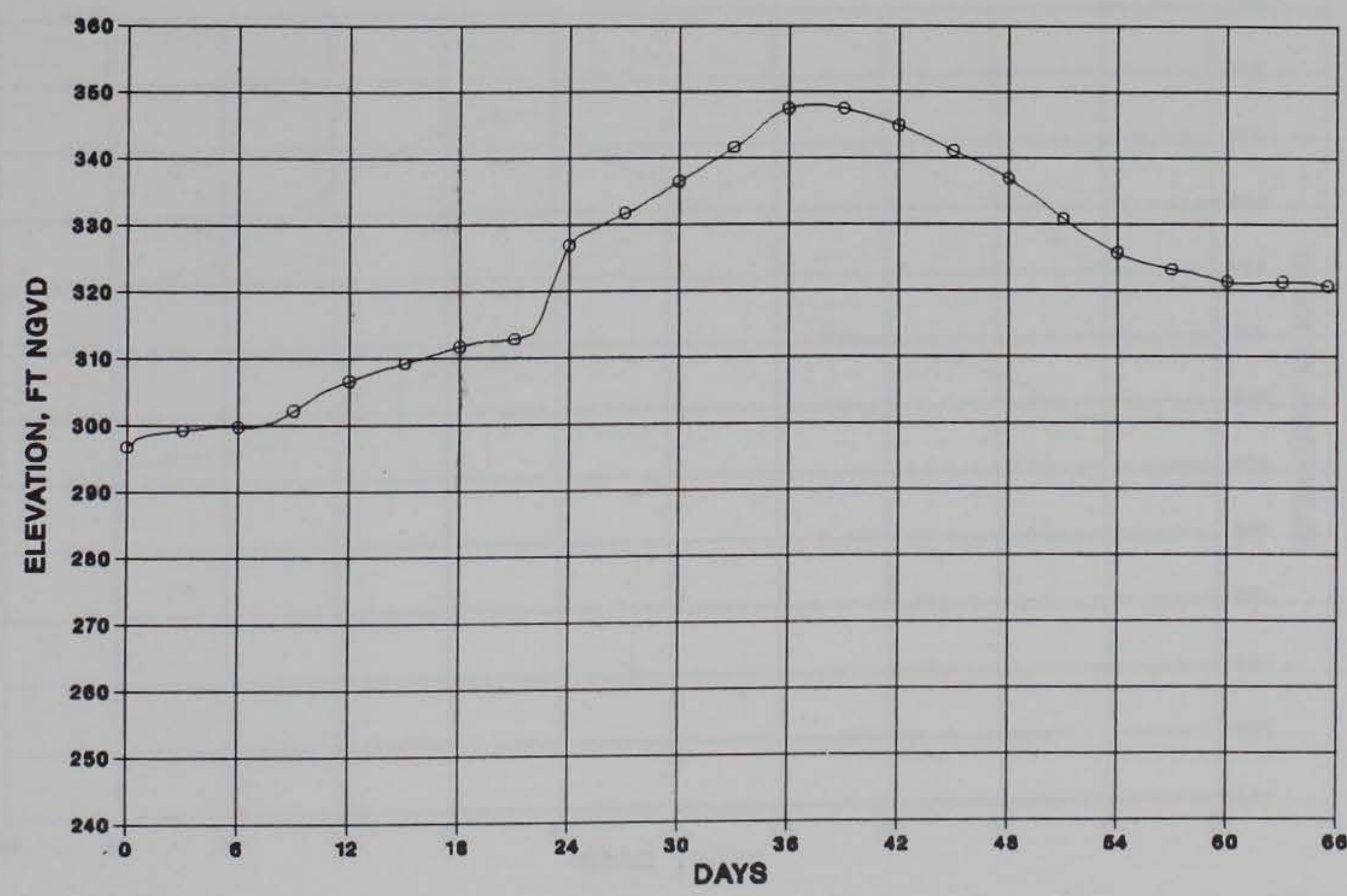

b. Elevation

Figure 52. Results at Metropolis, IL, for Pickwick PMF Dam break 


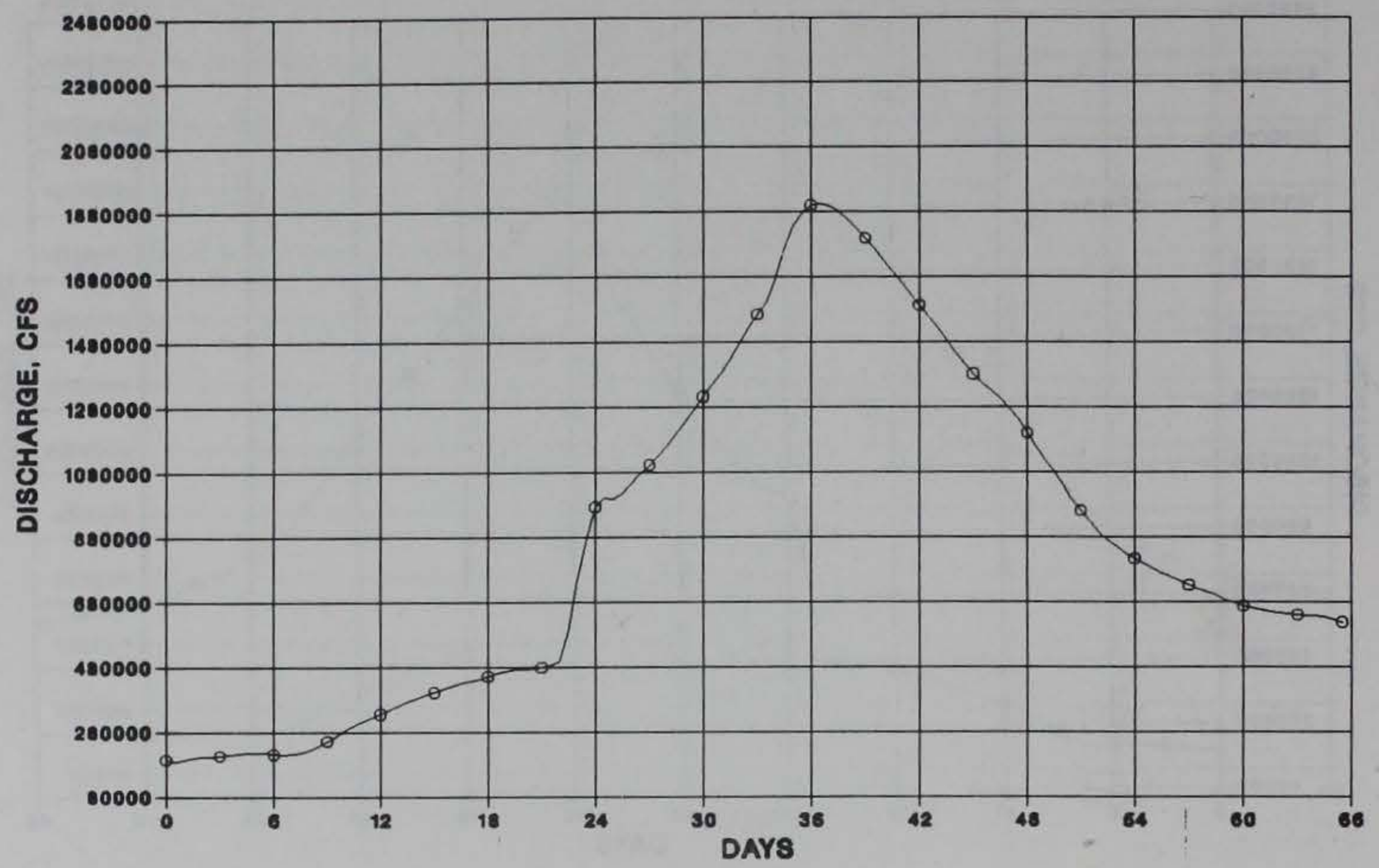

a. Discharge

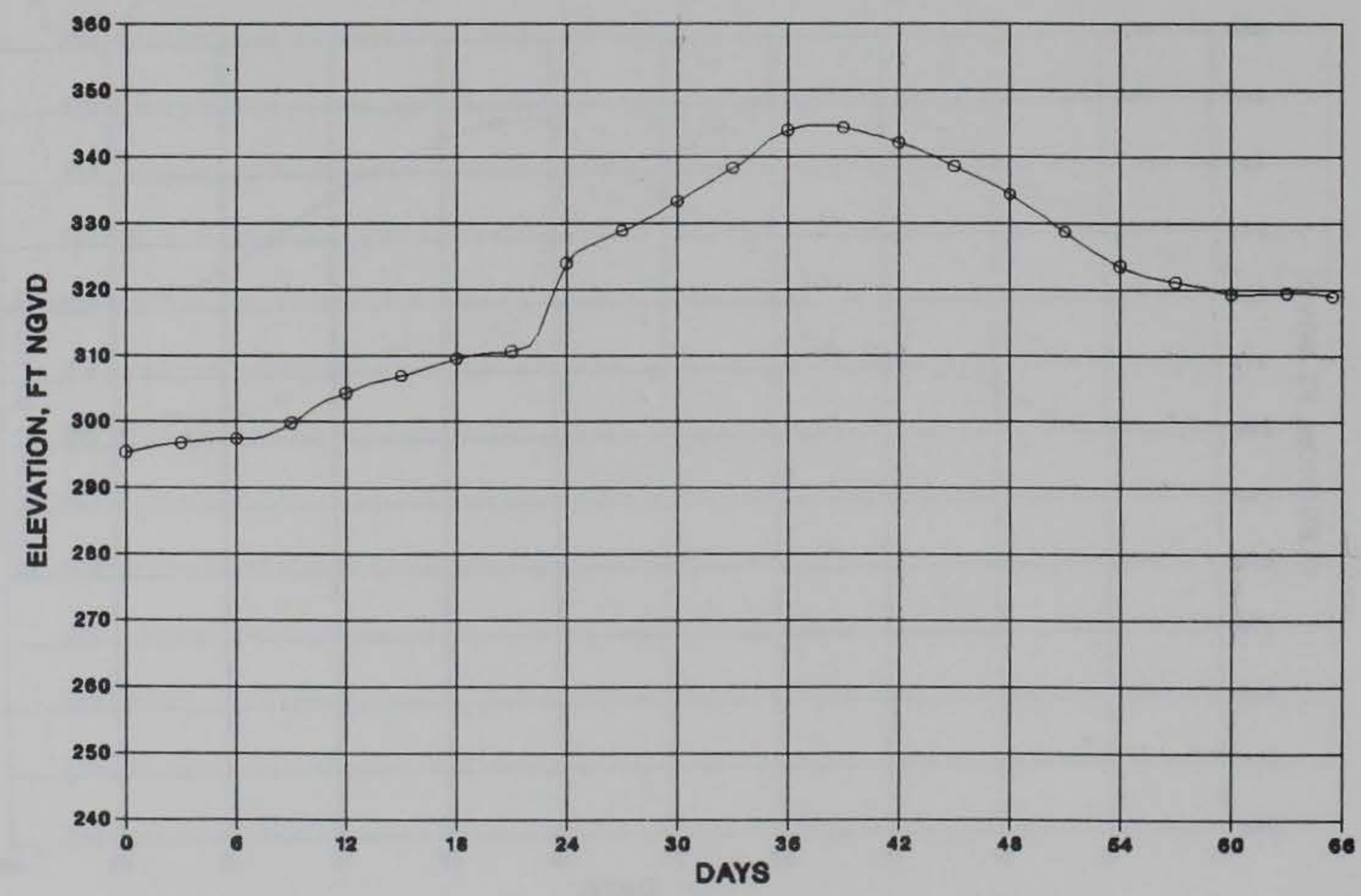

b. Elevation

Figure 53. Results at Joppa, IL, for Pickwick PMF Dam break 


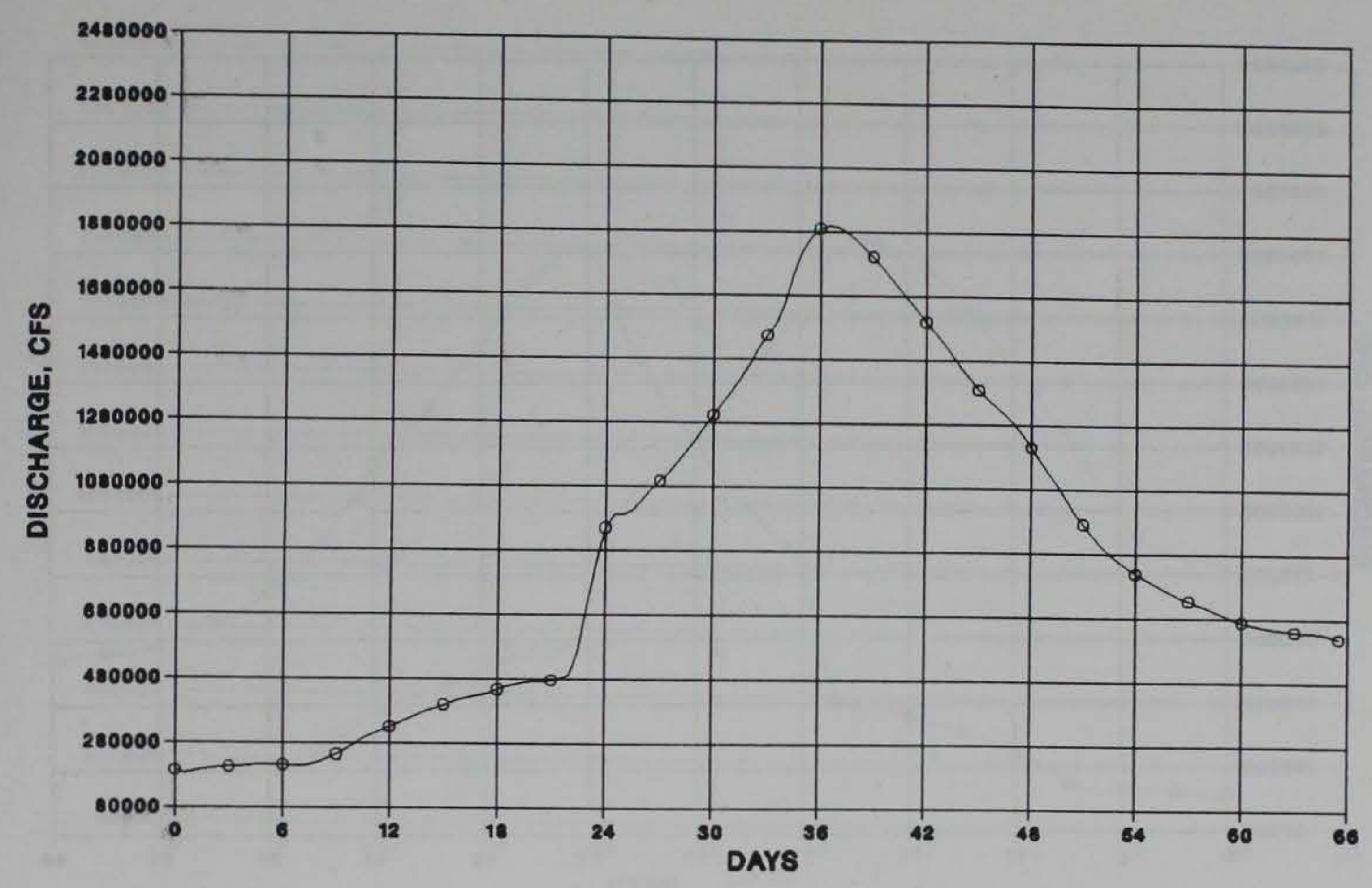

a. Discharge

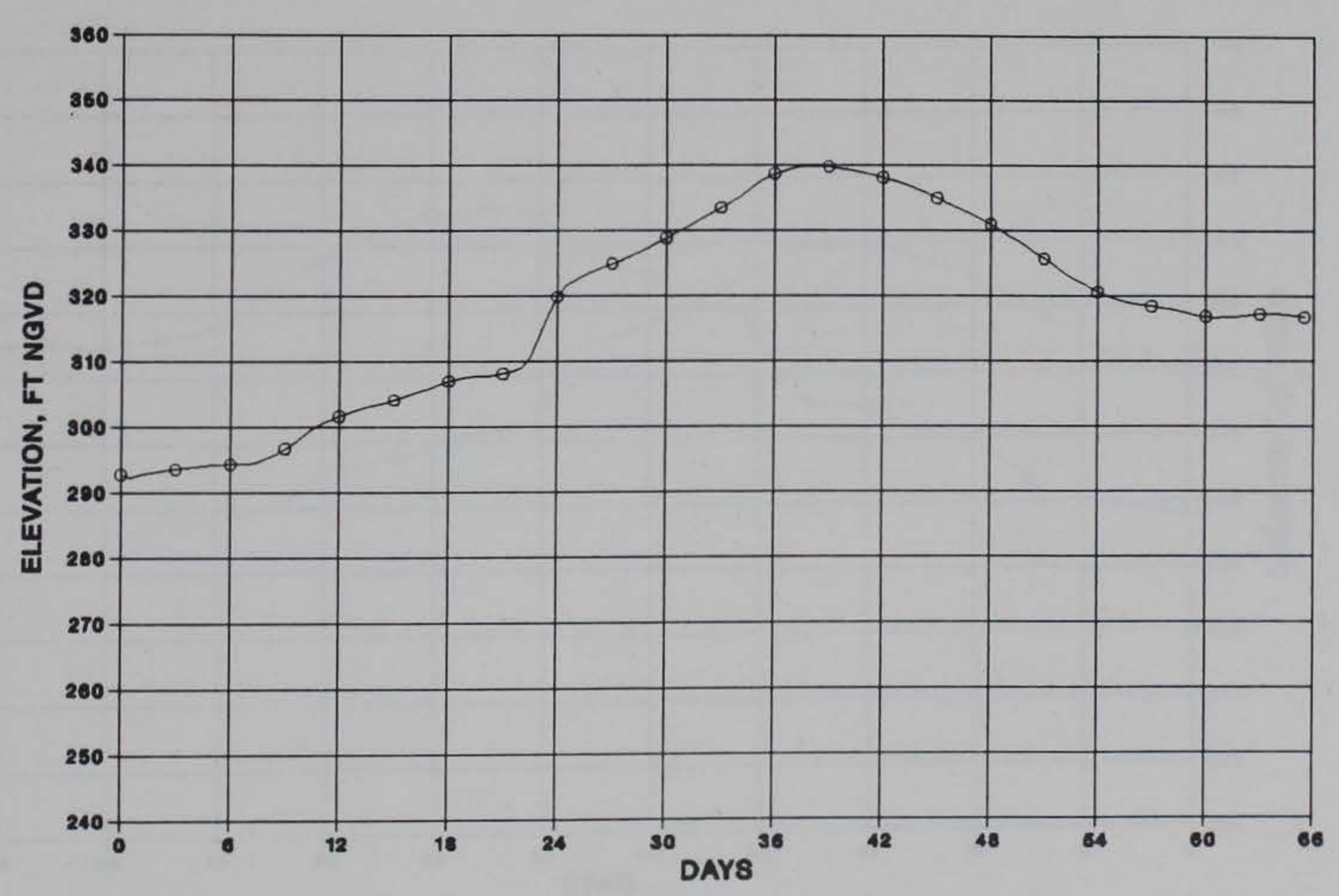

b. Elevation

Figure 54. Results at 0lmstead, IL, for Pickwick PMF Dam break 


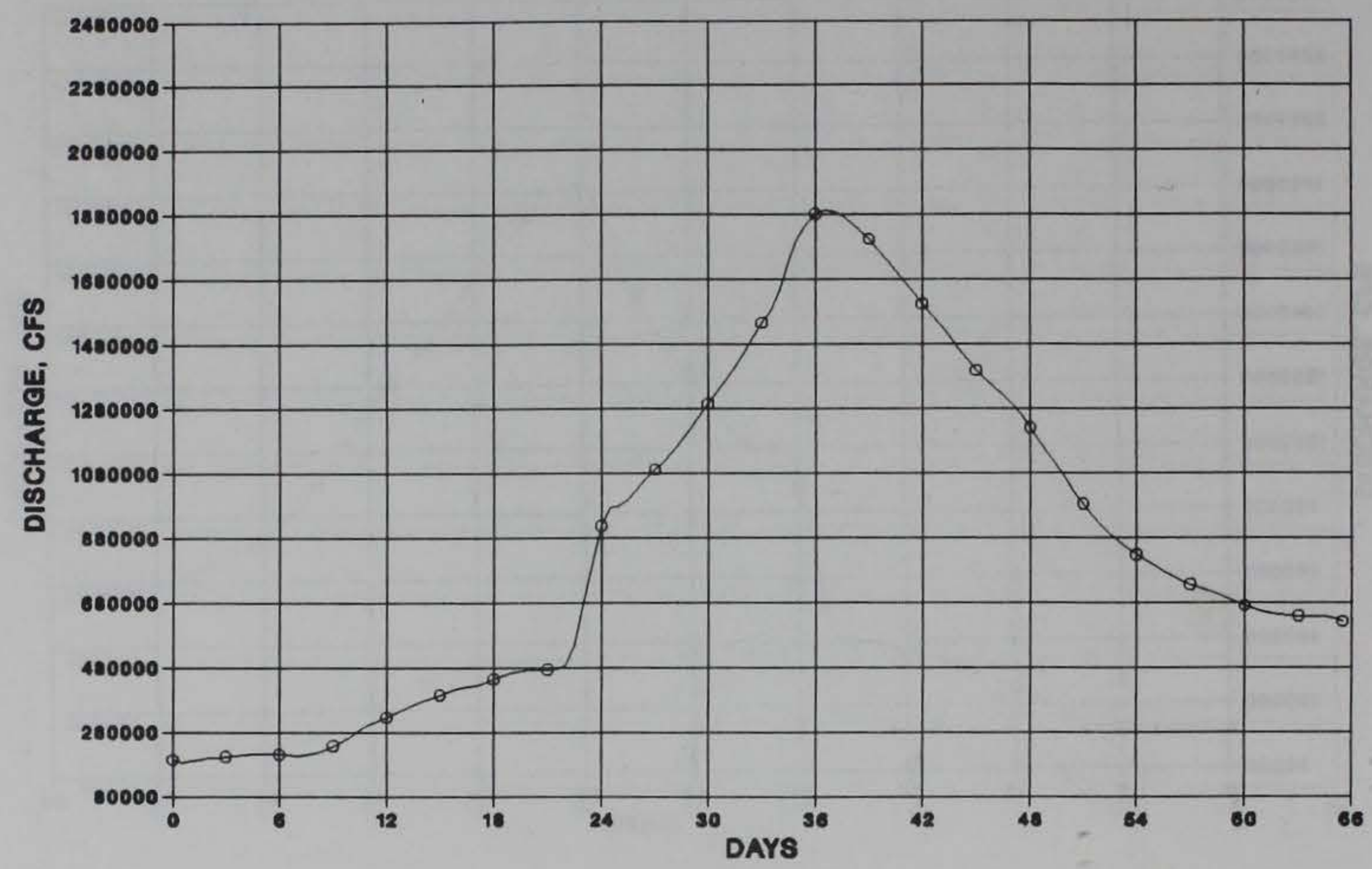

a. Discharge

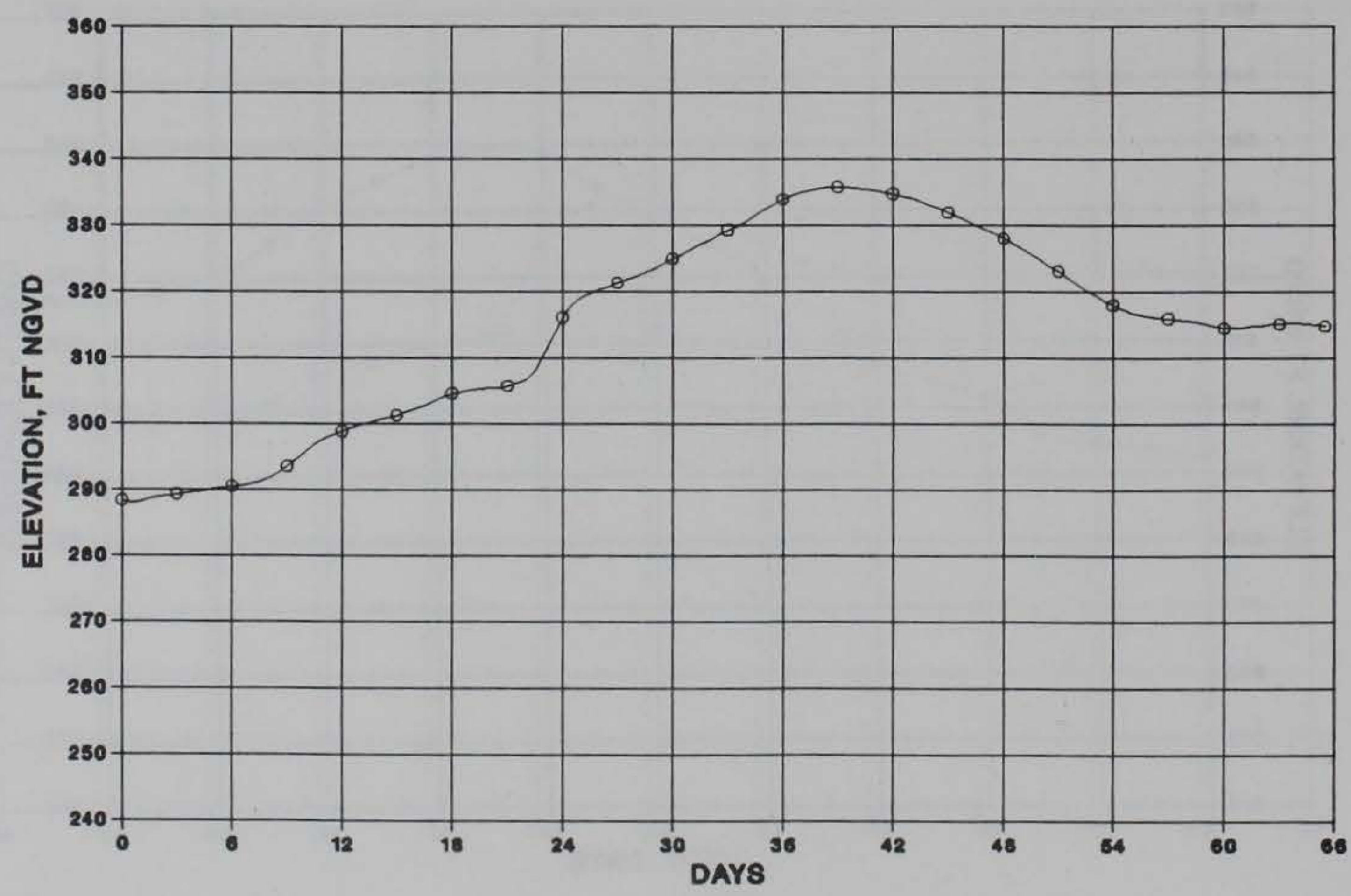

b. Elevation

Figure 55. Results at Cairo, IL, for Pickwick PMF Dam break 


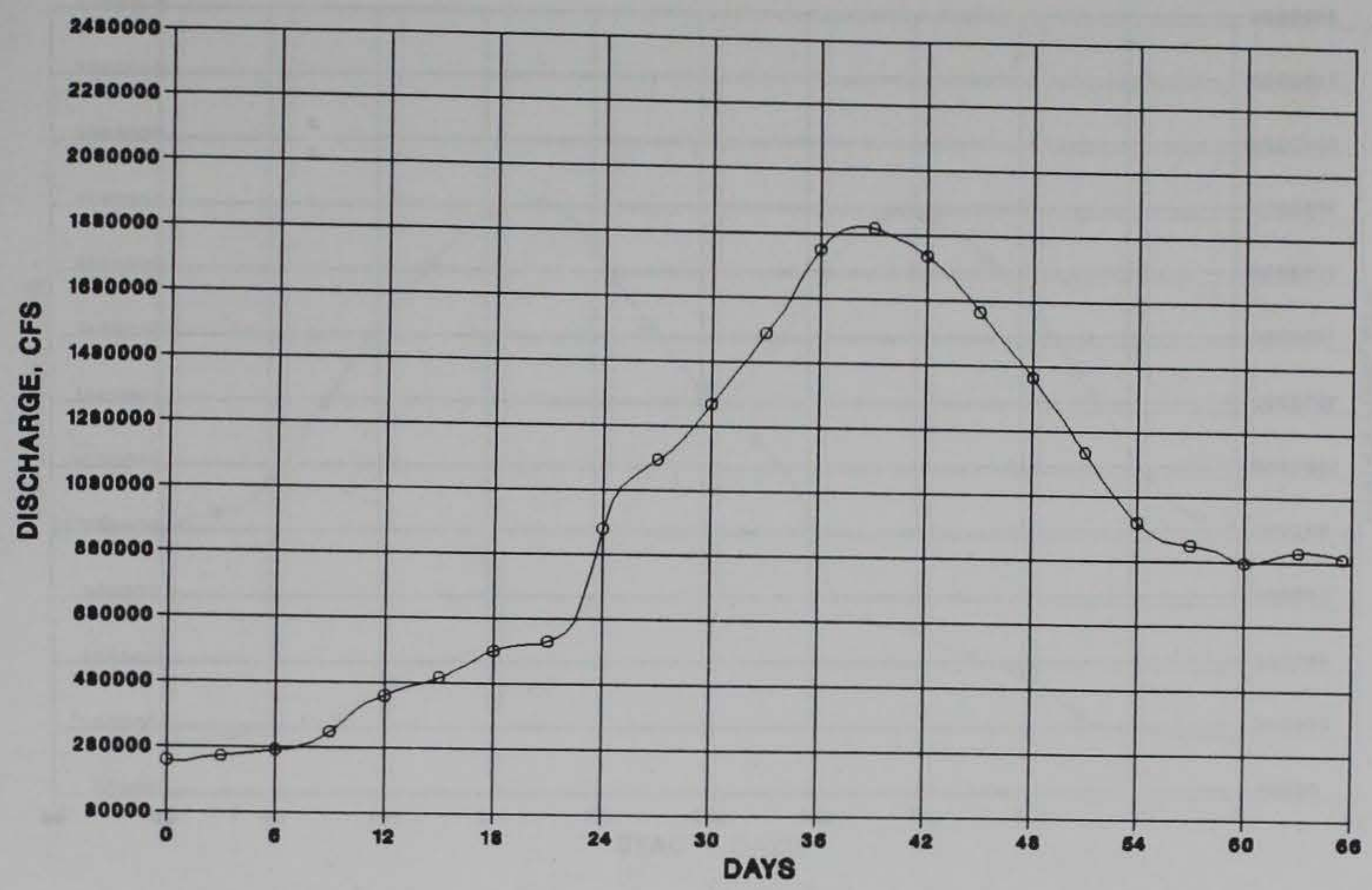

a. Discharge

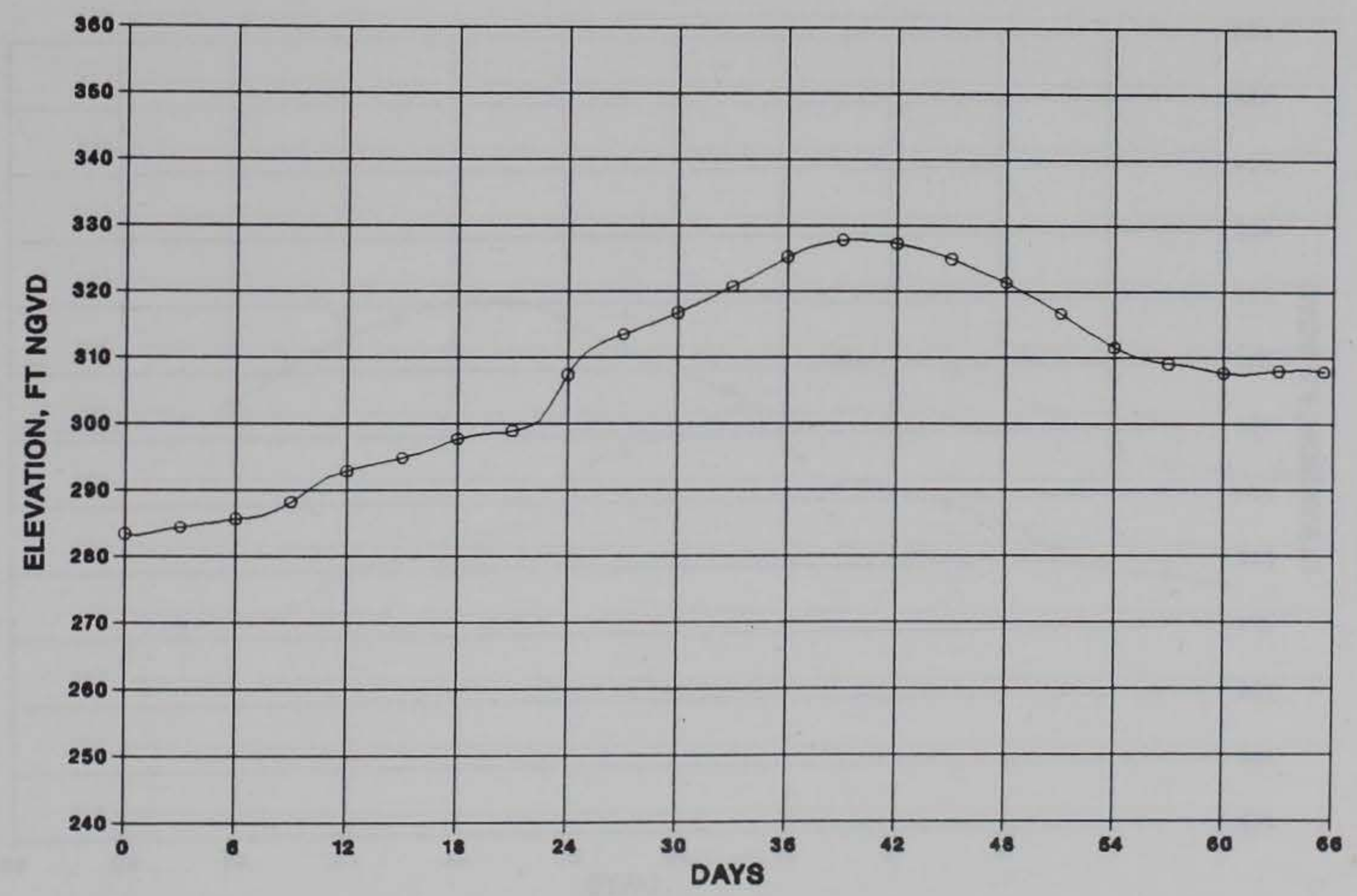

b. Elevation

Figure 56. Results at Columbus, KY, for Pickwick PMF Dam break 


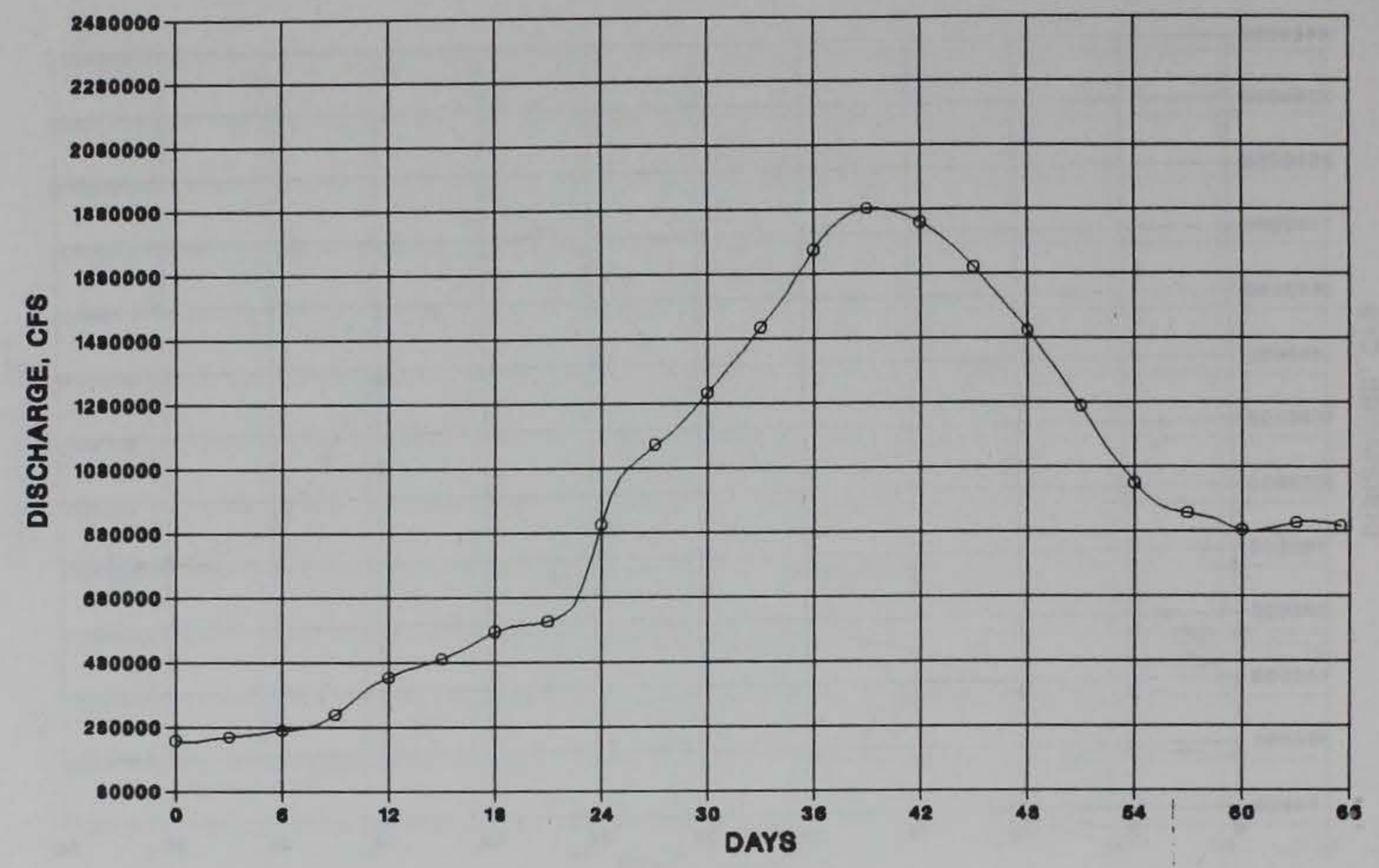

a. Discharge

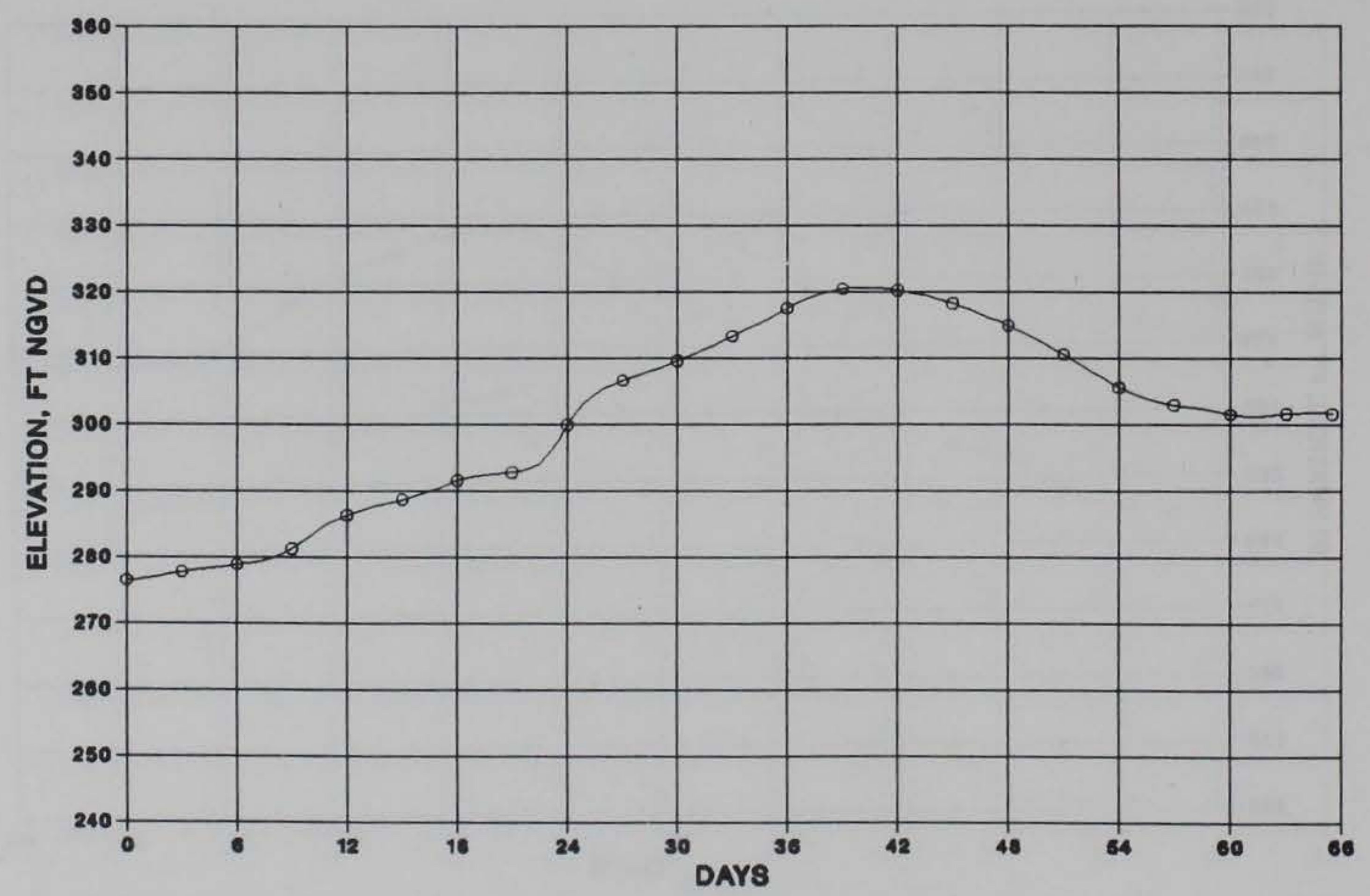

b. Elevation

Figure 57. Results at Hickman, KY, for Pickwick PMF Dam break 


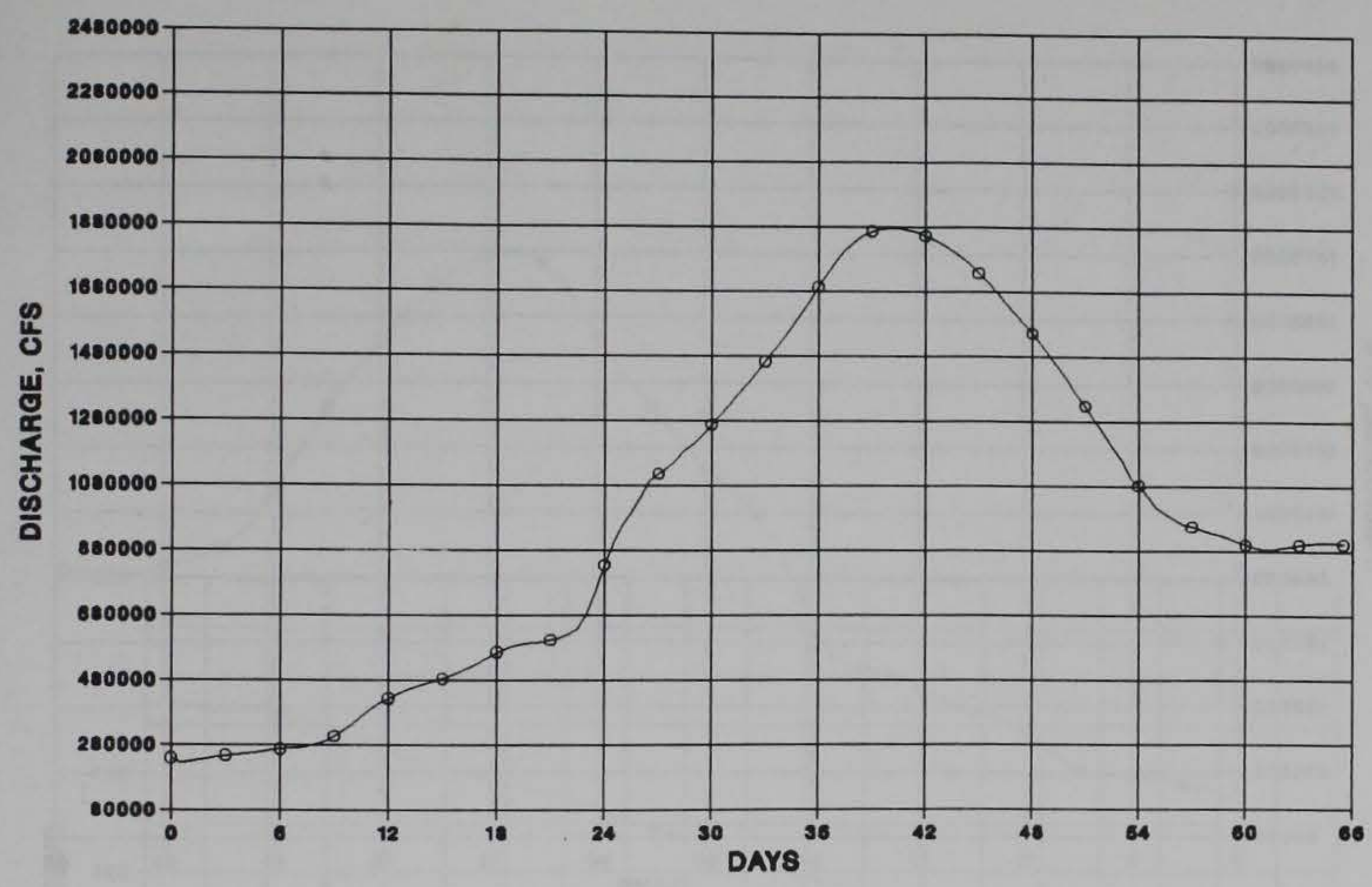

a. Discharge

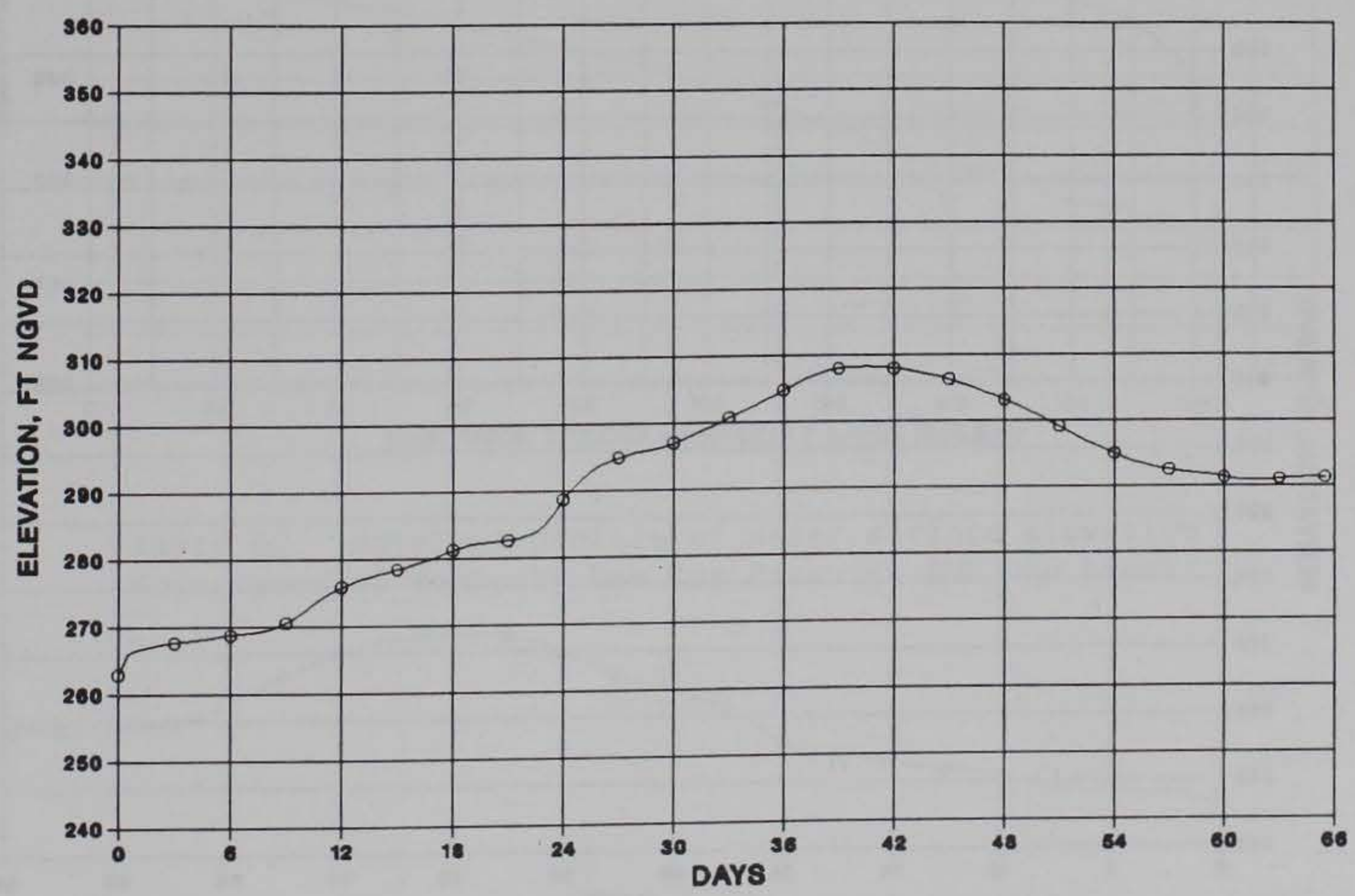

b. Elevation

Figure 58. Results at New Madrid, MO, for Pickwick PMF Dam break 


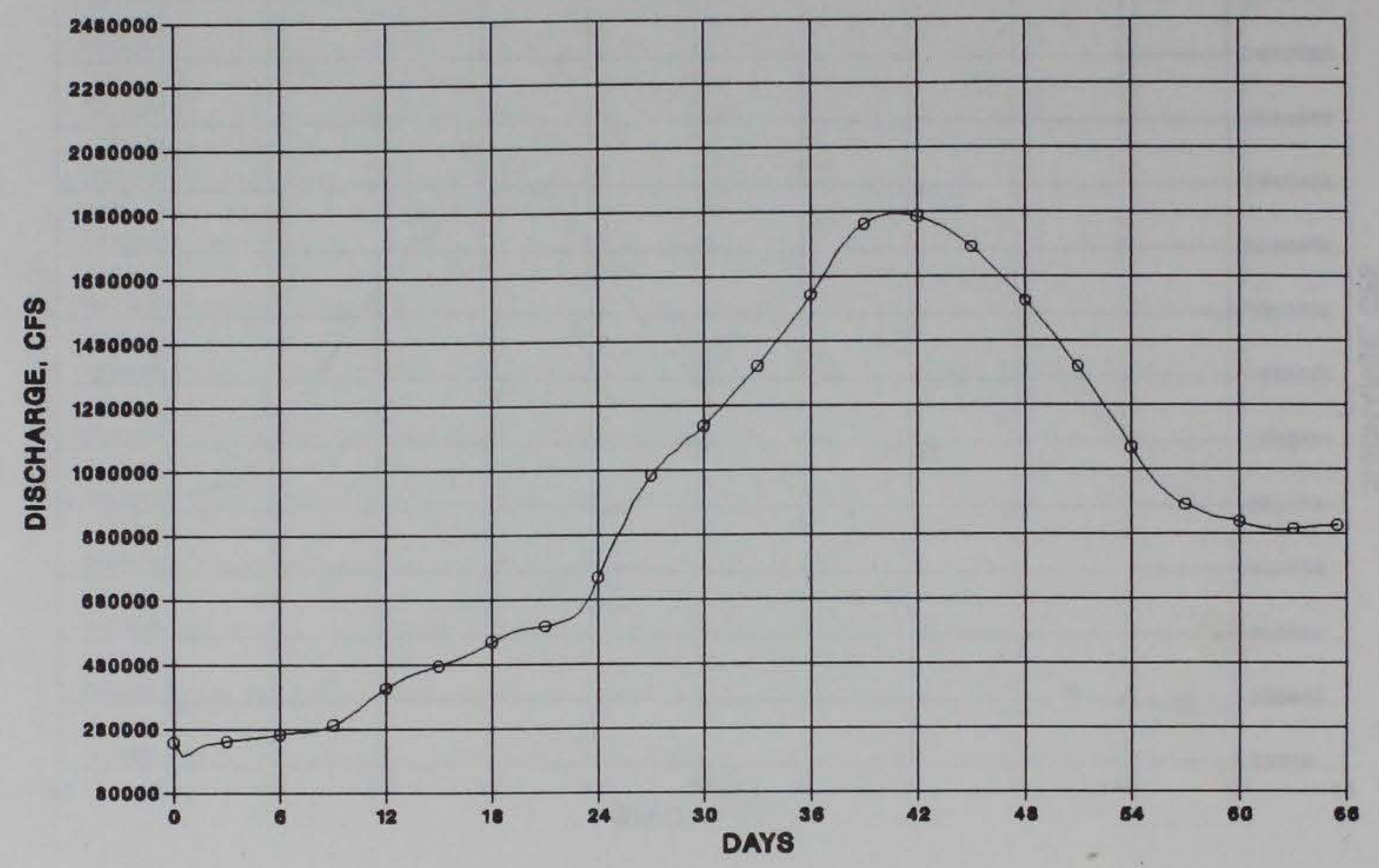

a. Discharge

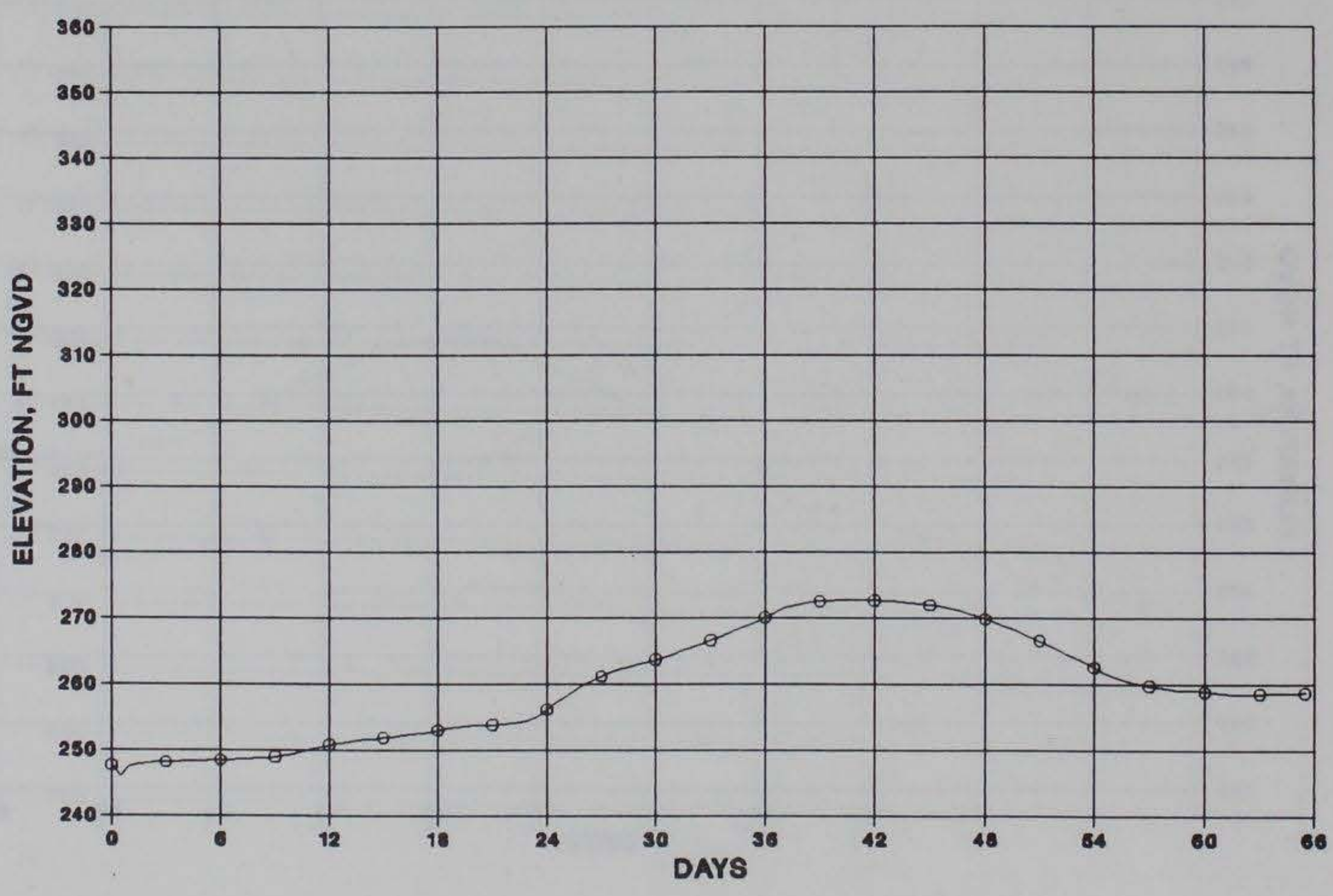

b. Elevation

Figure 59. Results at Caruthersville, MO, for Pickwick PMF Dam break 


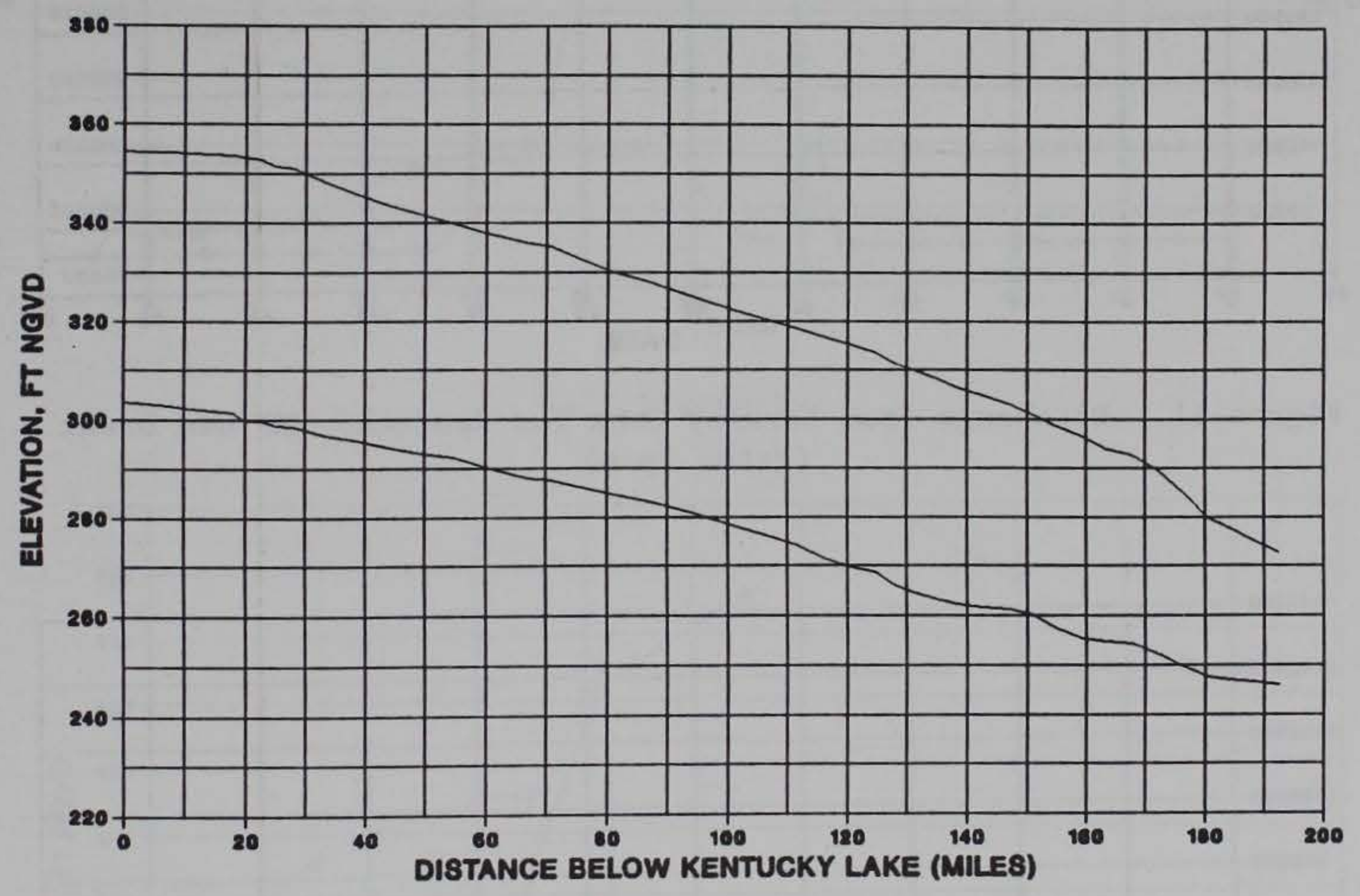

Figure 60. Envelope profile of water surface elevation downstream of Kentucky Dam for Pickwick PMF Dam break 


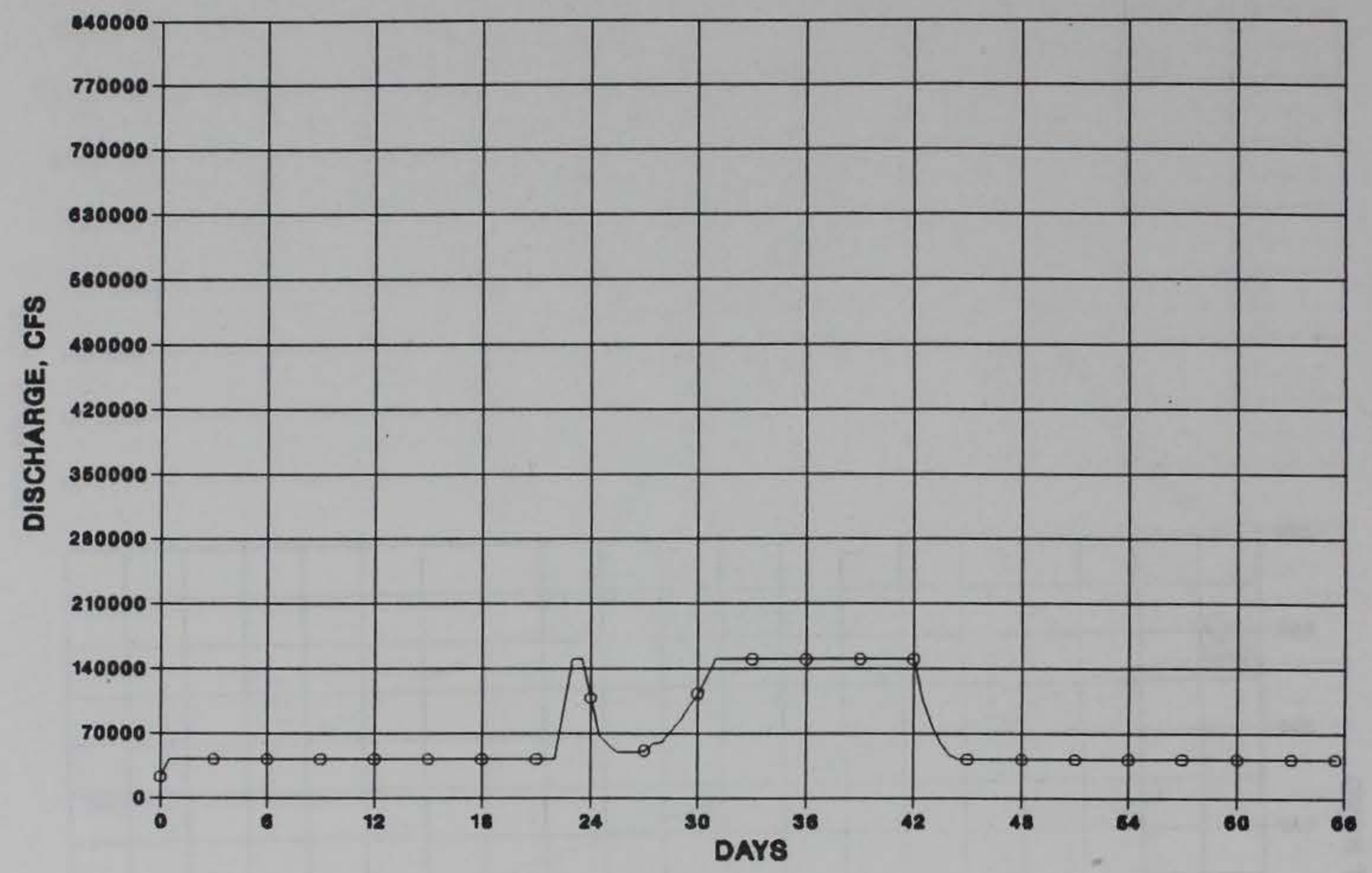

Figure 61. Discharge from Barkley Lake for Kentucky PMF dam break (gates open)

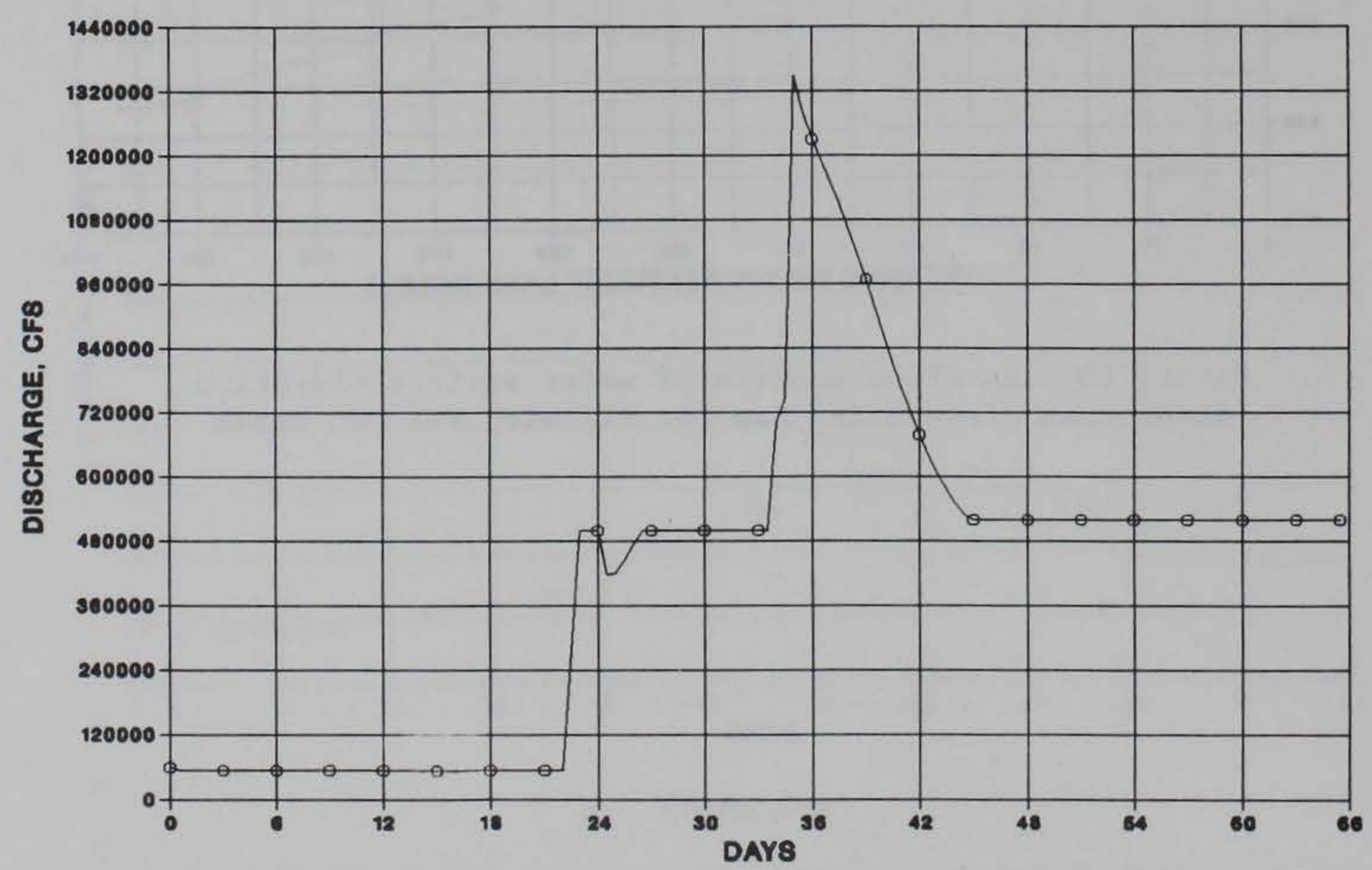

Figure 62. Discharge from Kentucky Lake for Kentucky PMF dam break (gates open) 


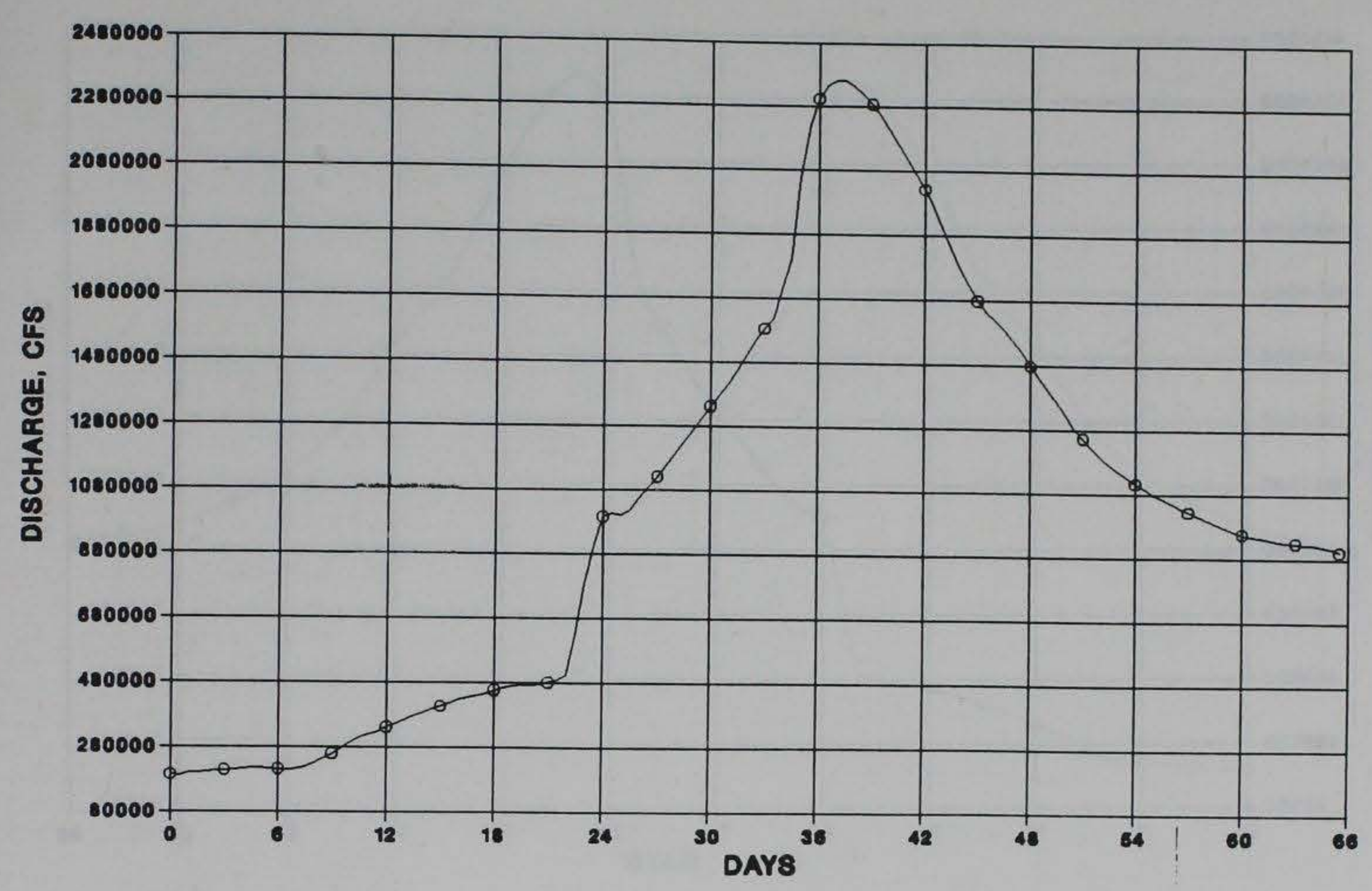

a. Discharge

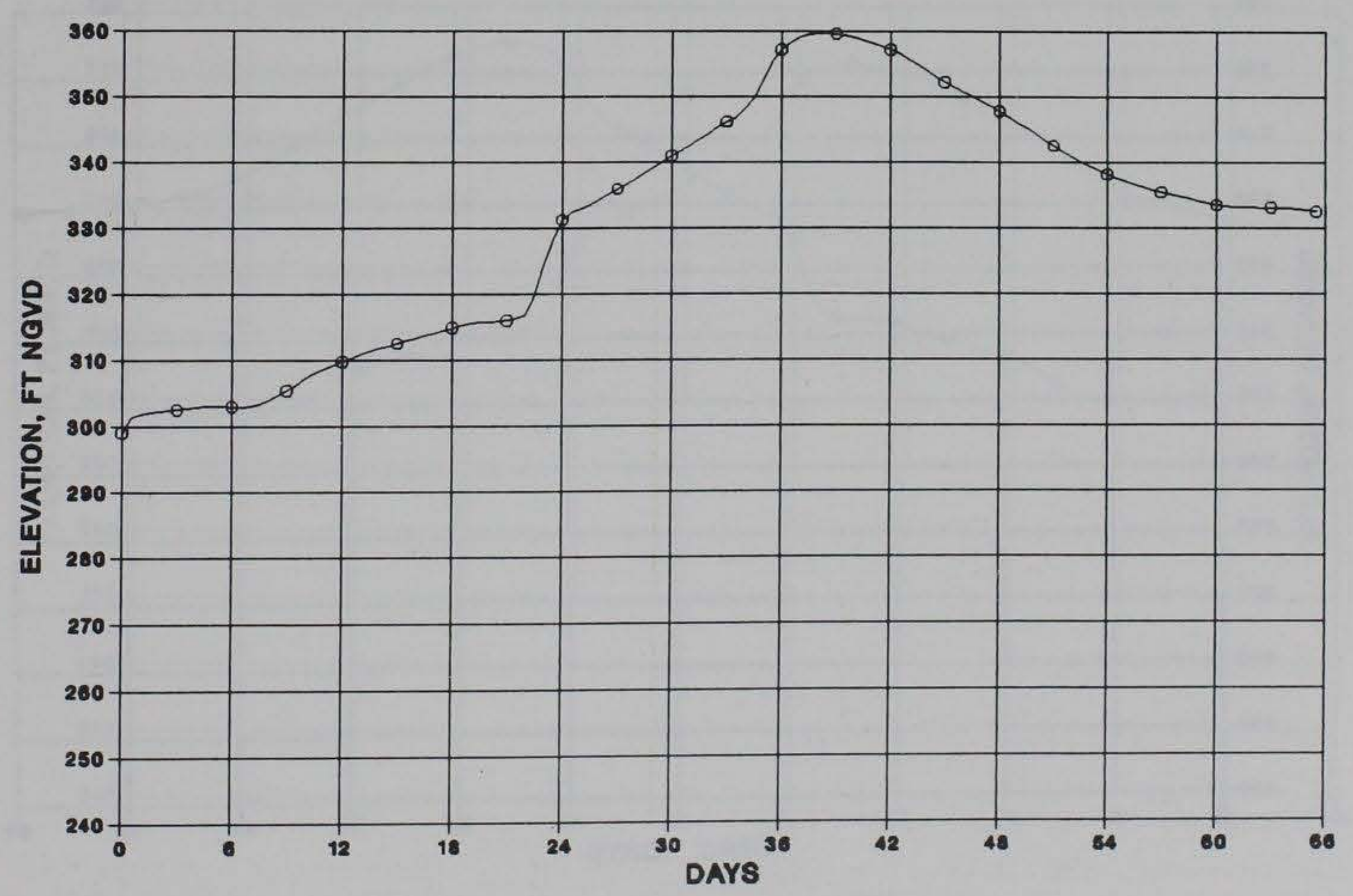

b. Elevation

Figure 63. Results at Paducah, KY, for Kentucky PMF dam break (gates open) 


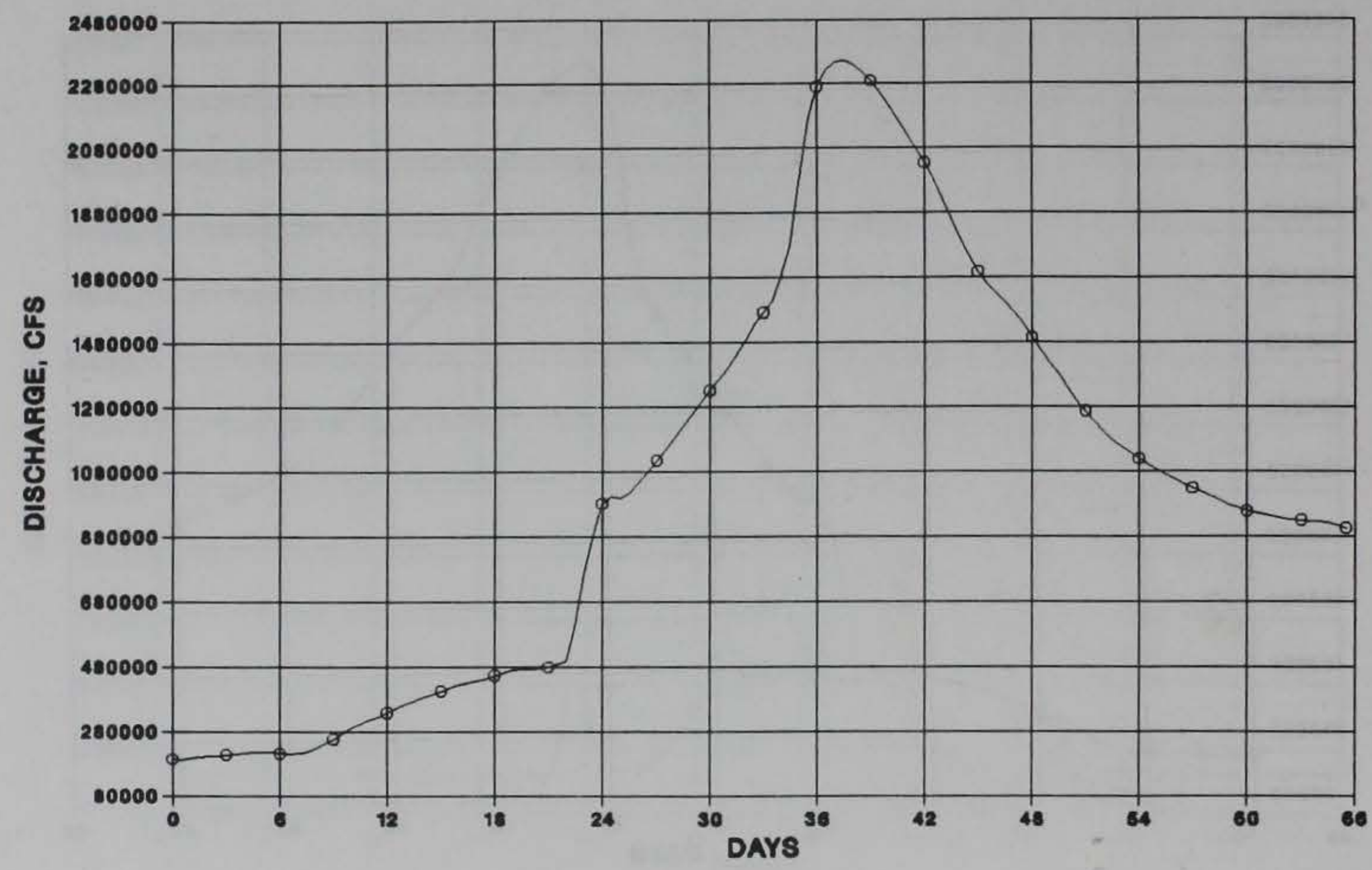

a. Discharge

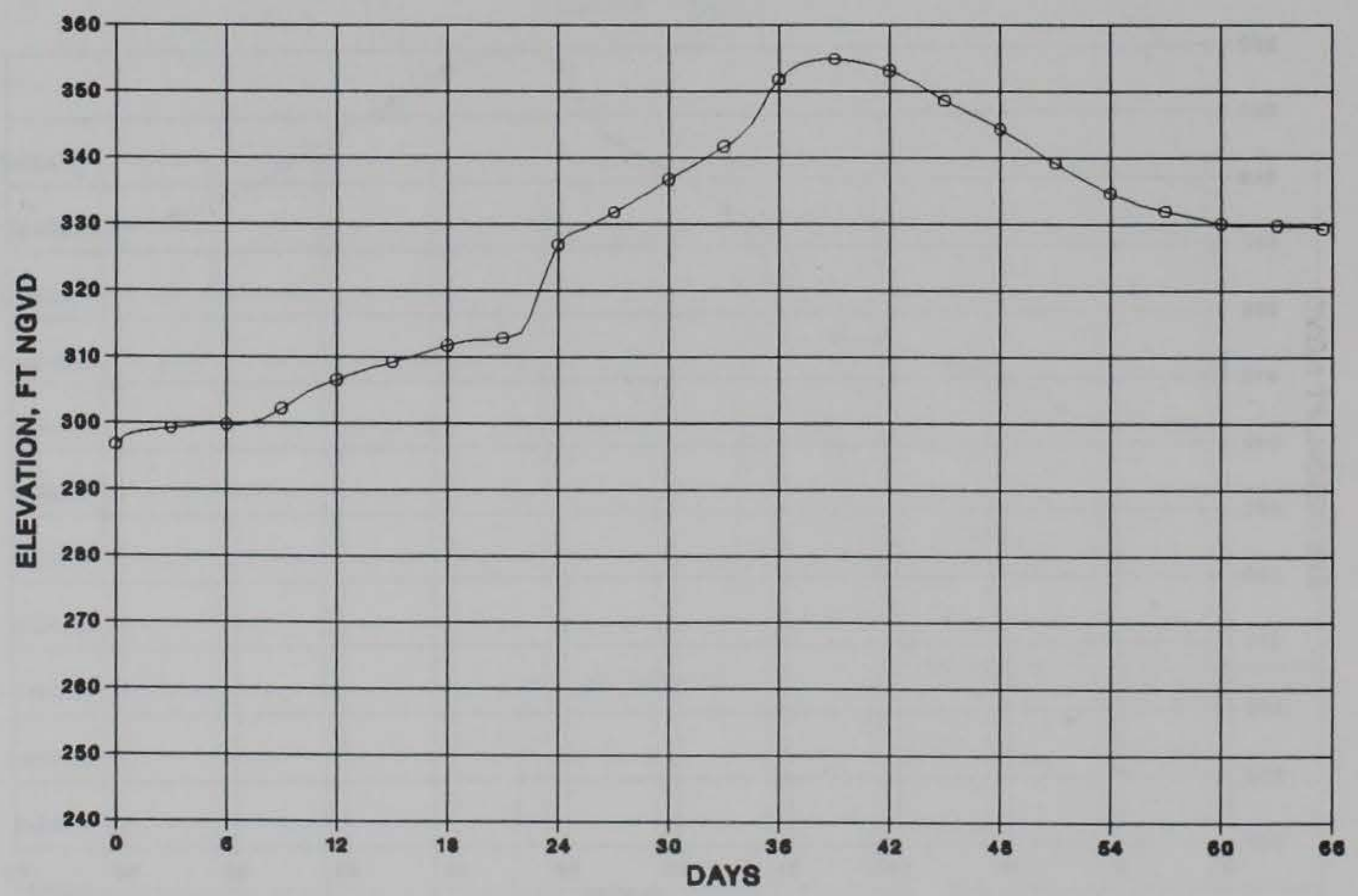

b. Elevation

Figure 64. Results at Metropolis, IL, for Kentucky PMF dam break (gates open) 


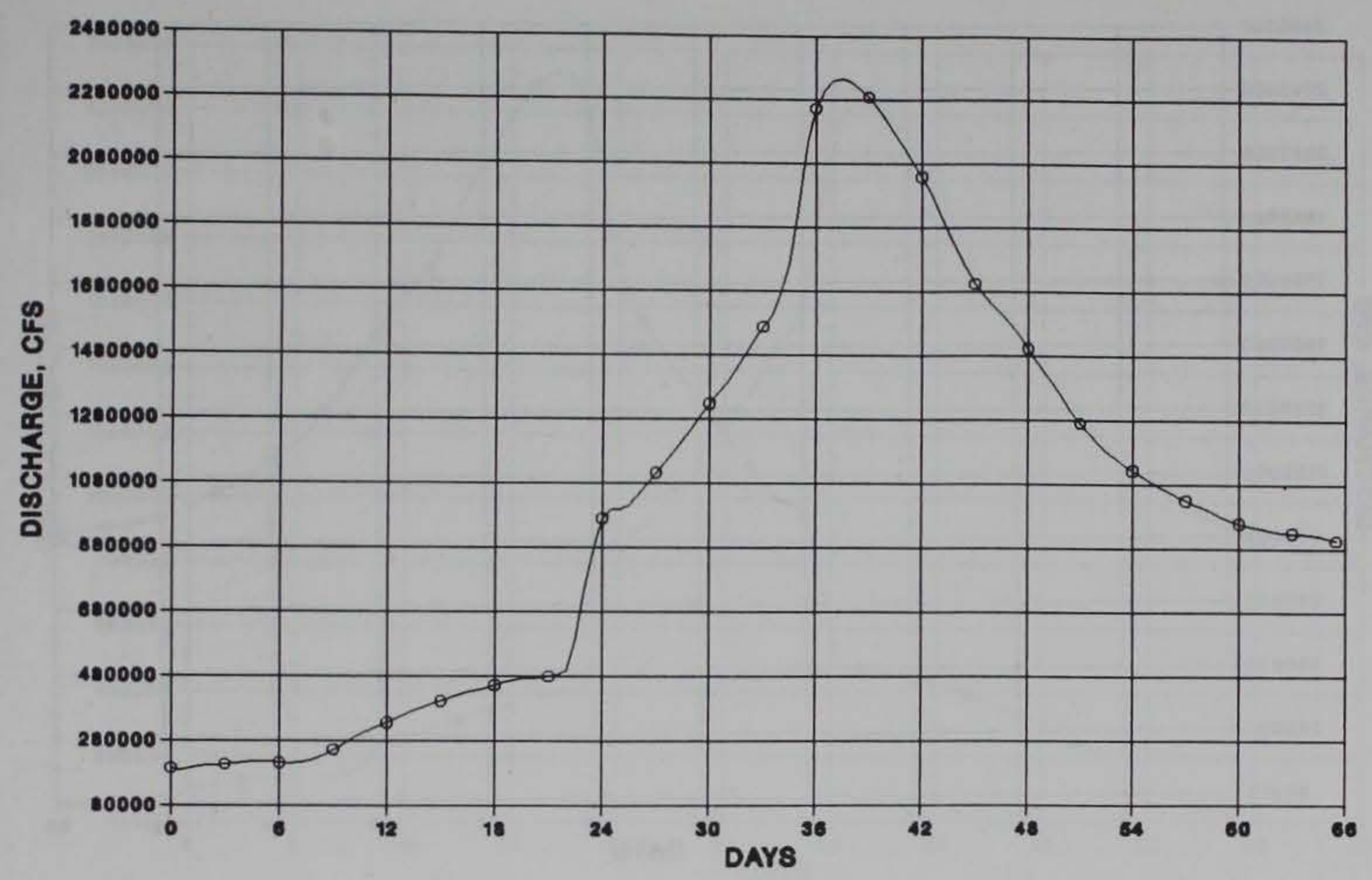

a. Discharge

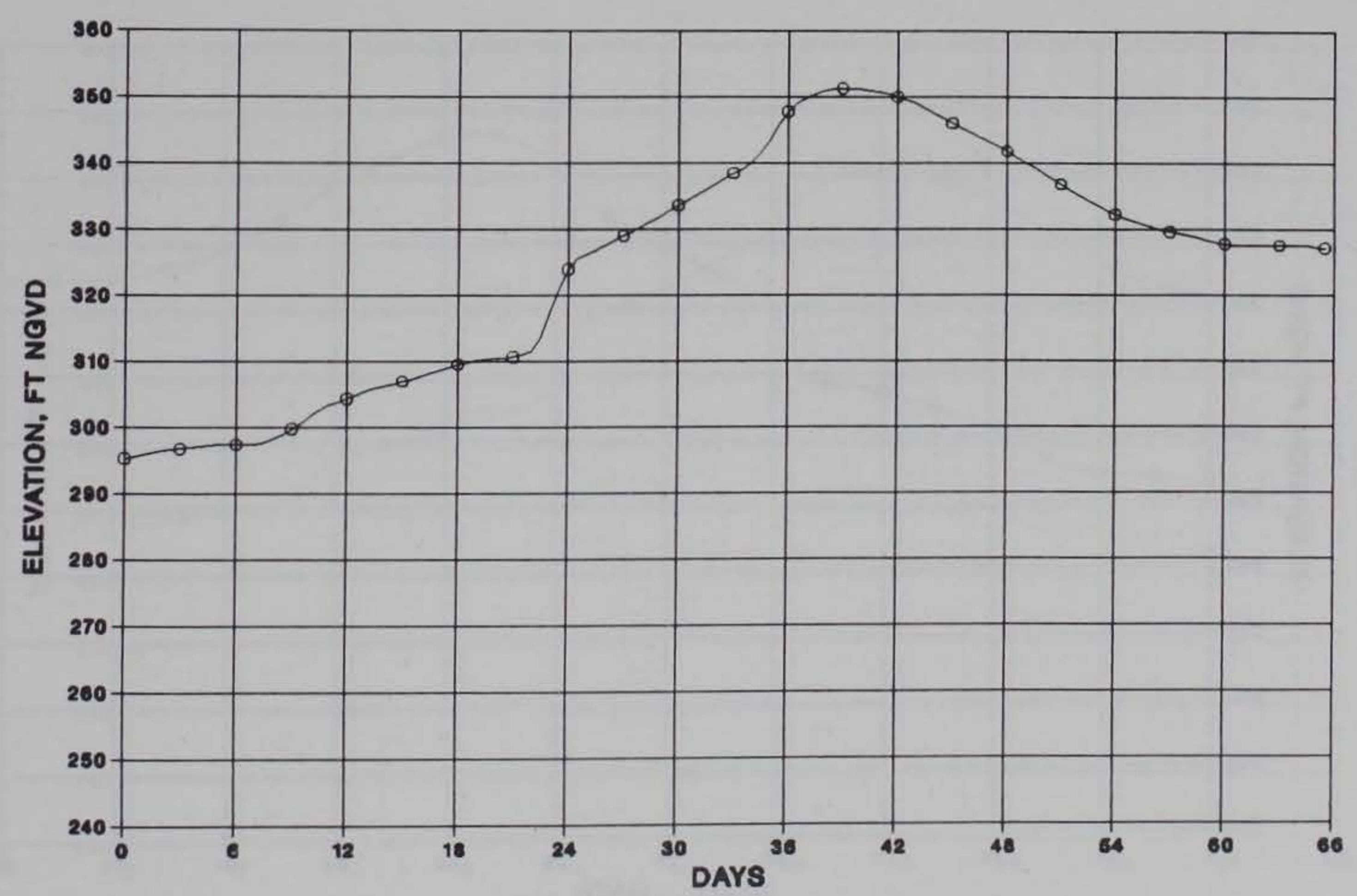

b. Elevation

Figure 65. Results at Joppa, IL, for Kentucky PMF dam break (gates open) 


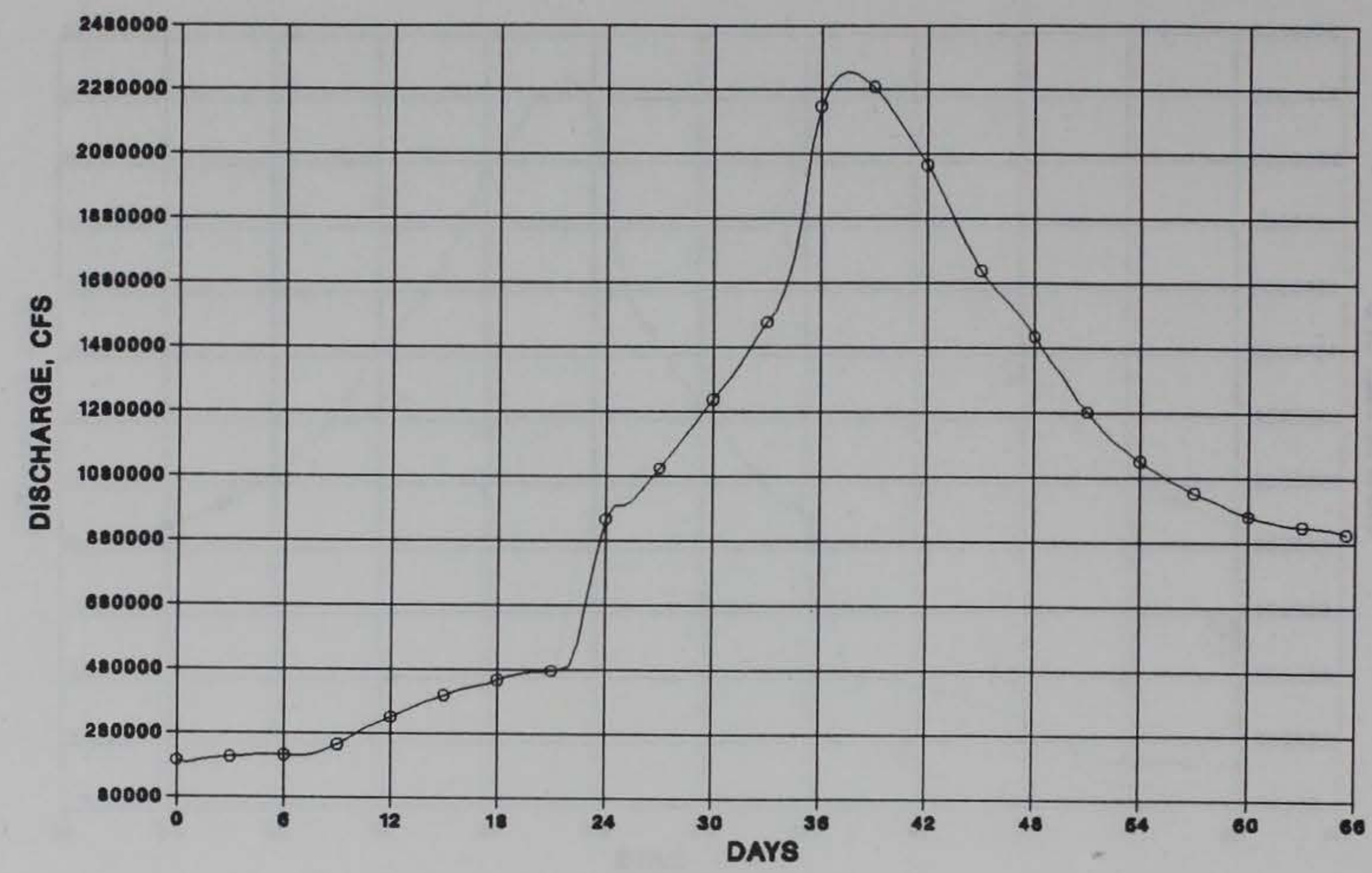

a. Discharge

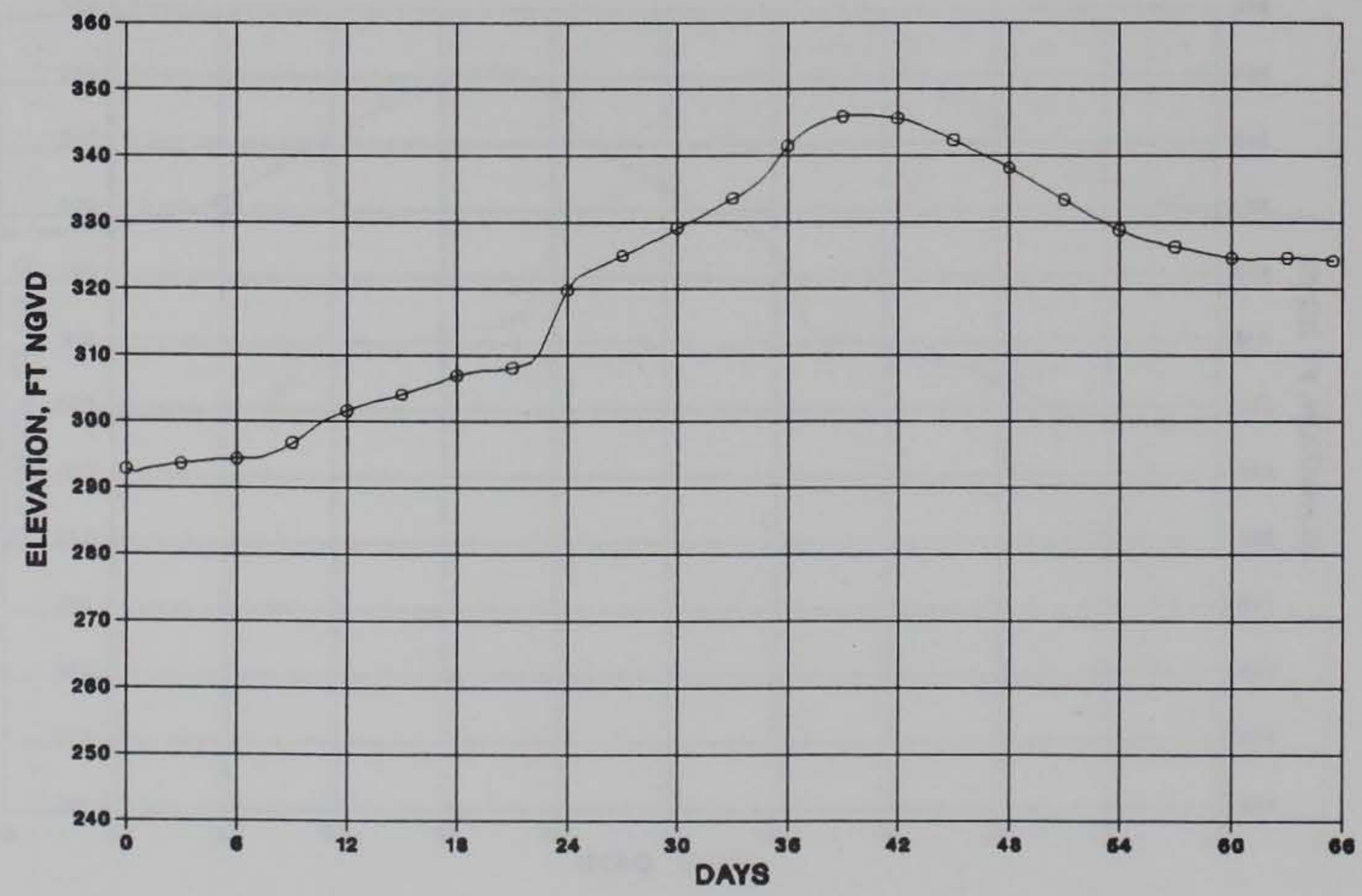

b. Elevation

Figure 66. Results at 01mstead, IL, for Kentucky PMF dam break (gates open) 


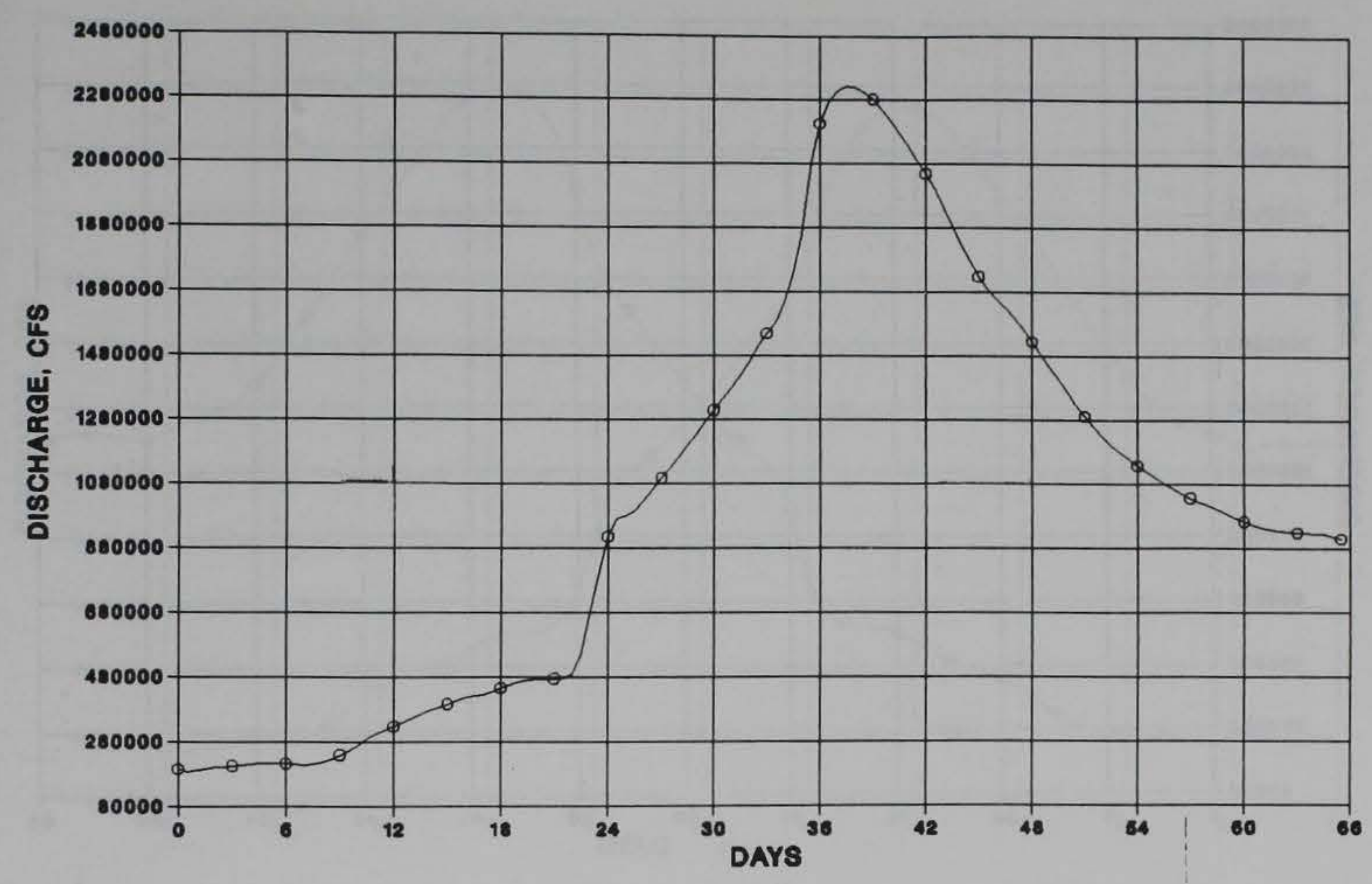

a. Discharge

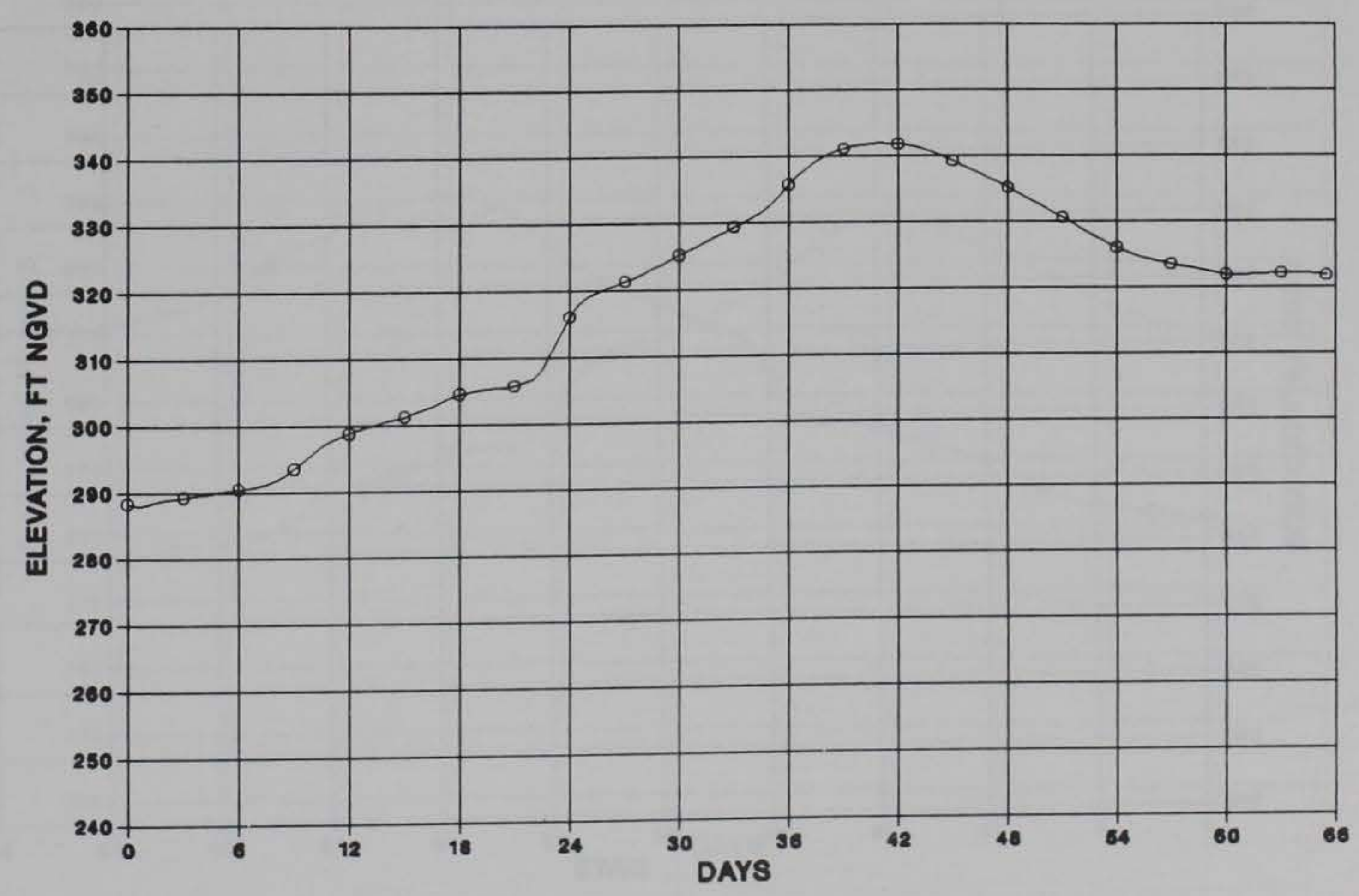

b. Elevation

Figure 67. Results at Cairo, IL, for Kentucky PMF dam break (gates open) 


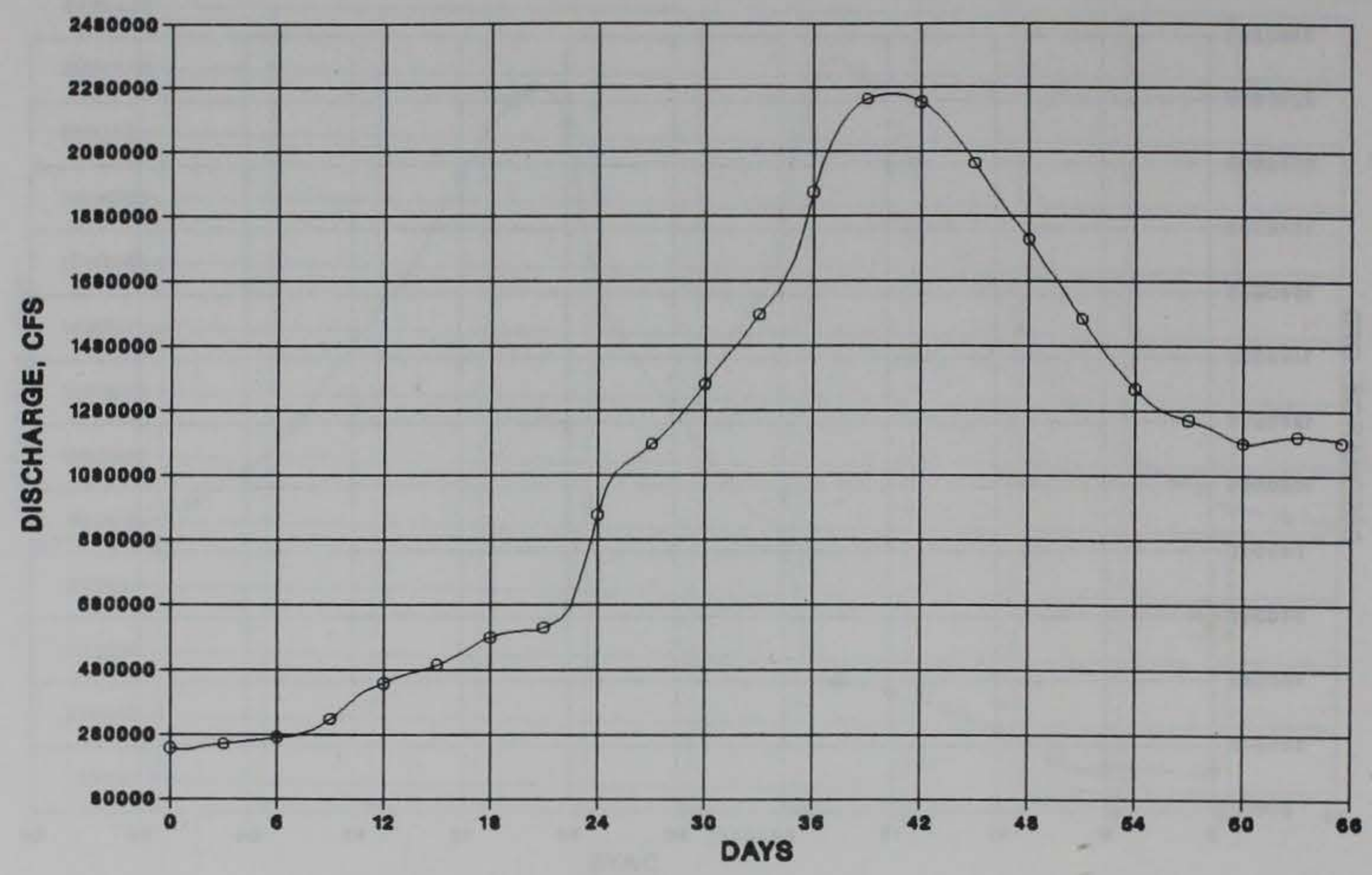

a. Discharge

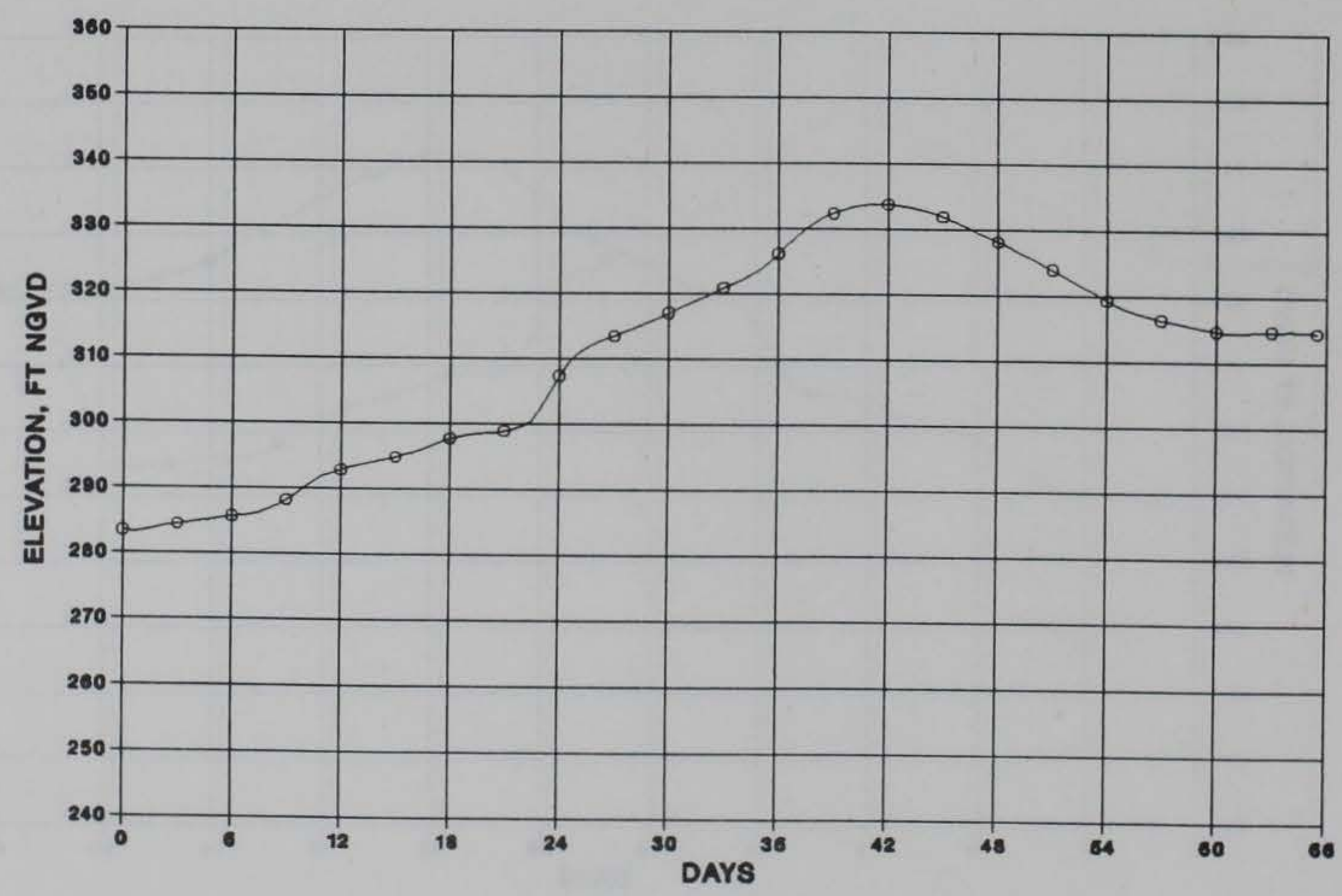

b. Elevation

Figure 68. Results at Columbus, KY, for Kentucky PMF dam break (gates open) 


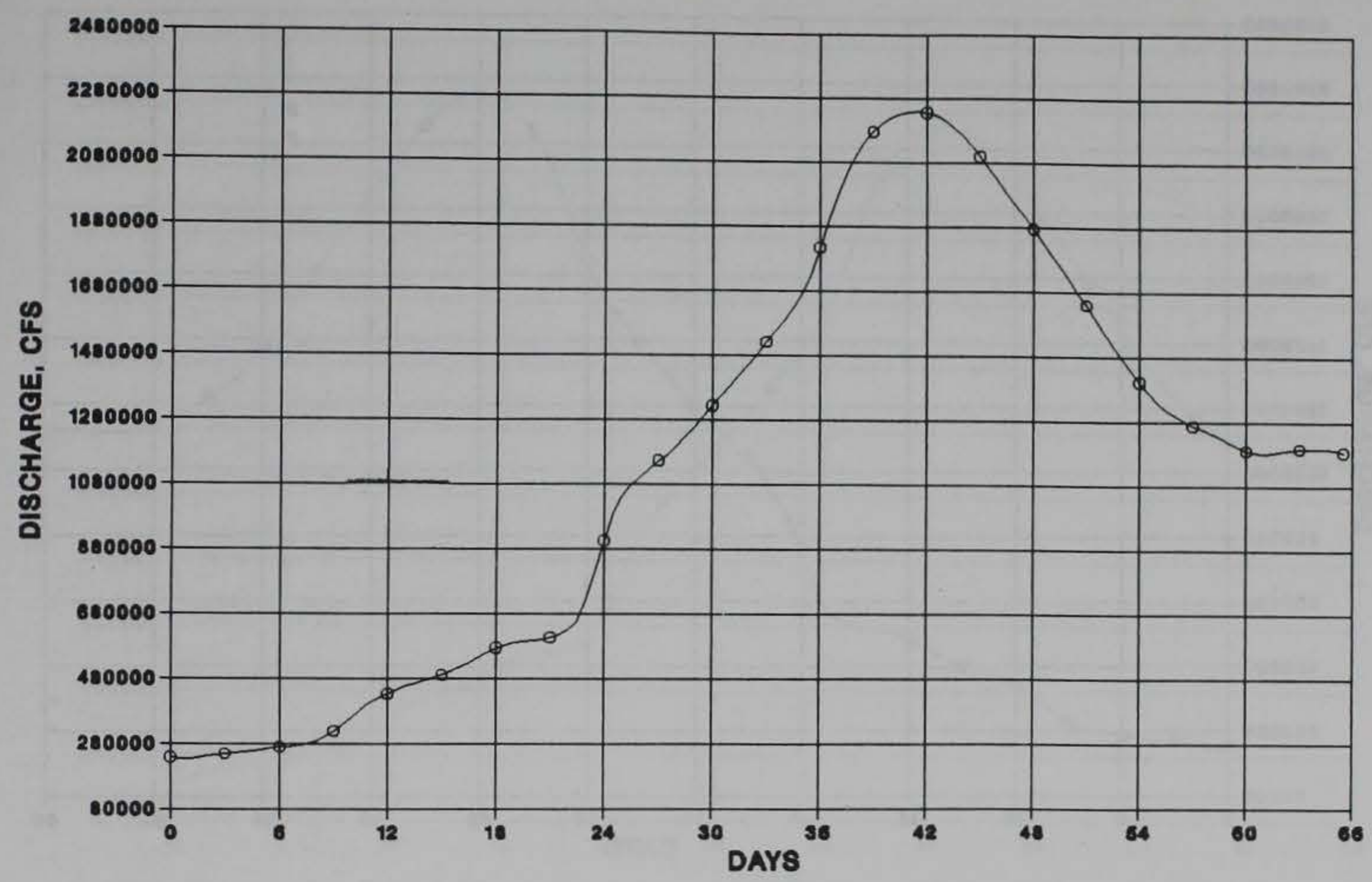

a. Discharge

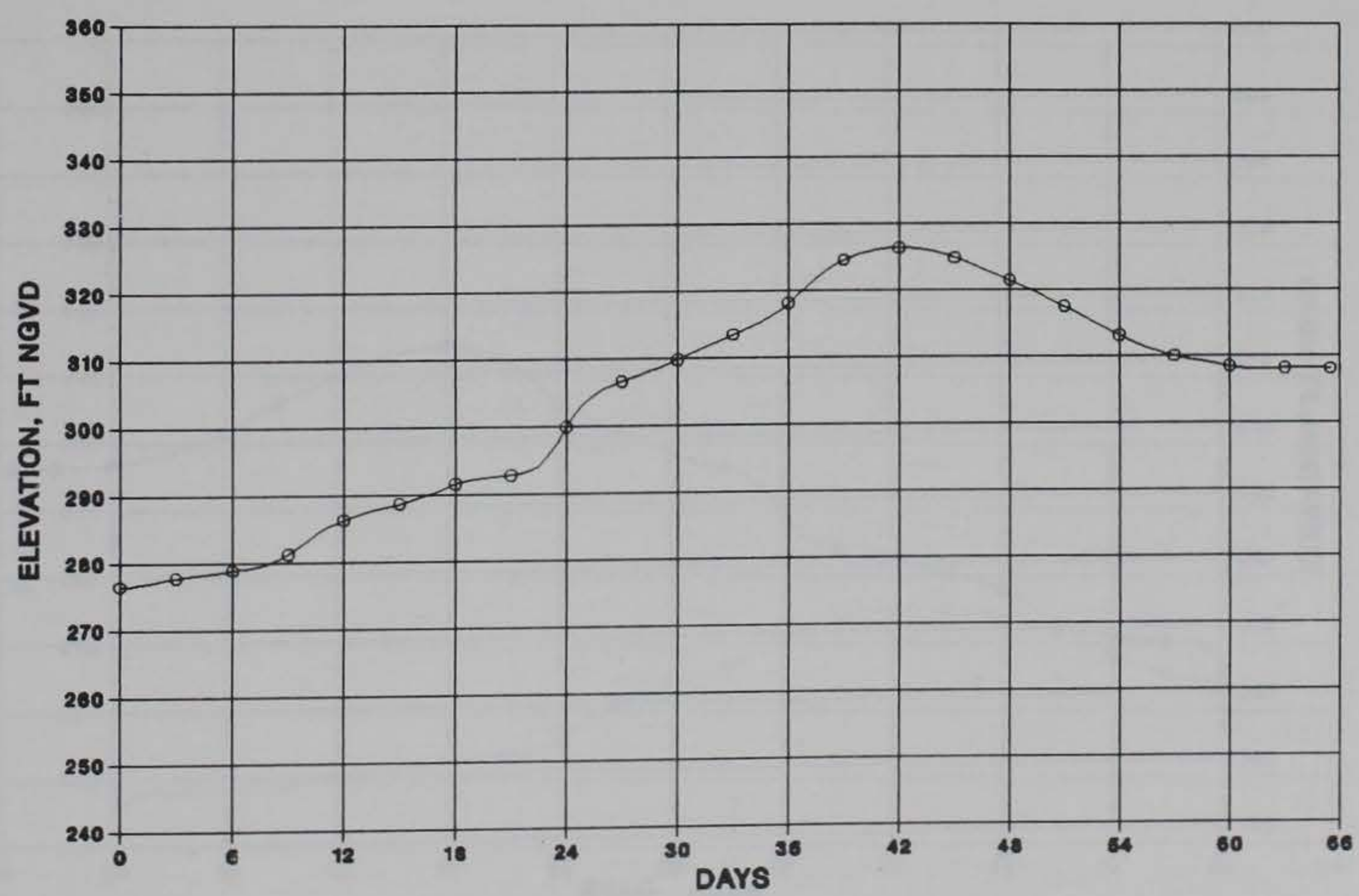

b. Elevation

Figure 69. Results at Hickman, KY, for Kentucky PMF dam break (gates open) 


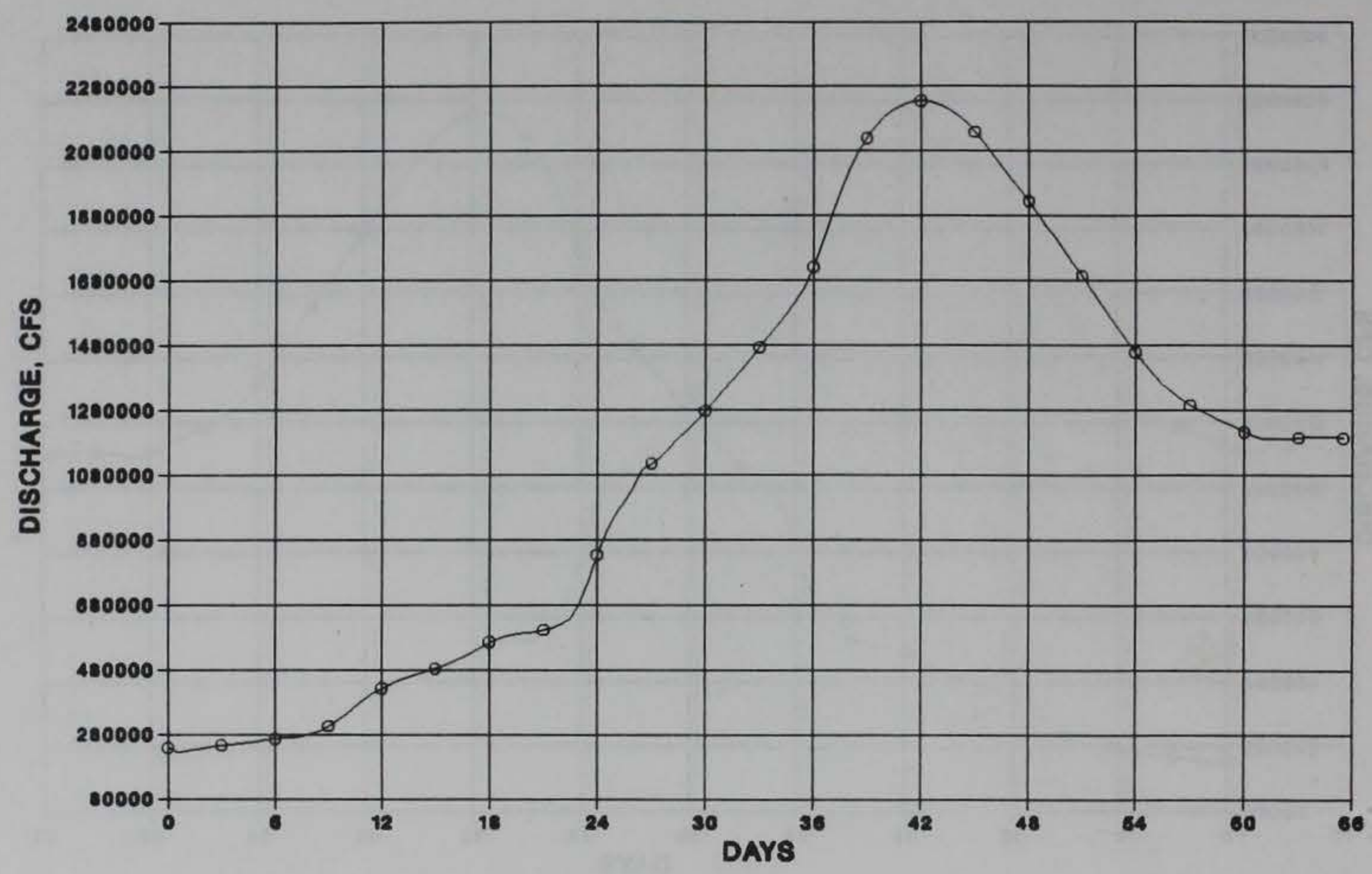

a. Discharge

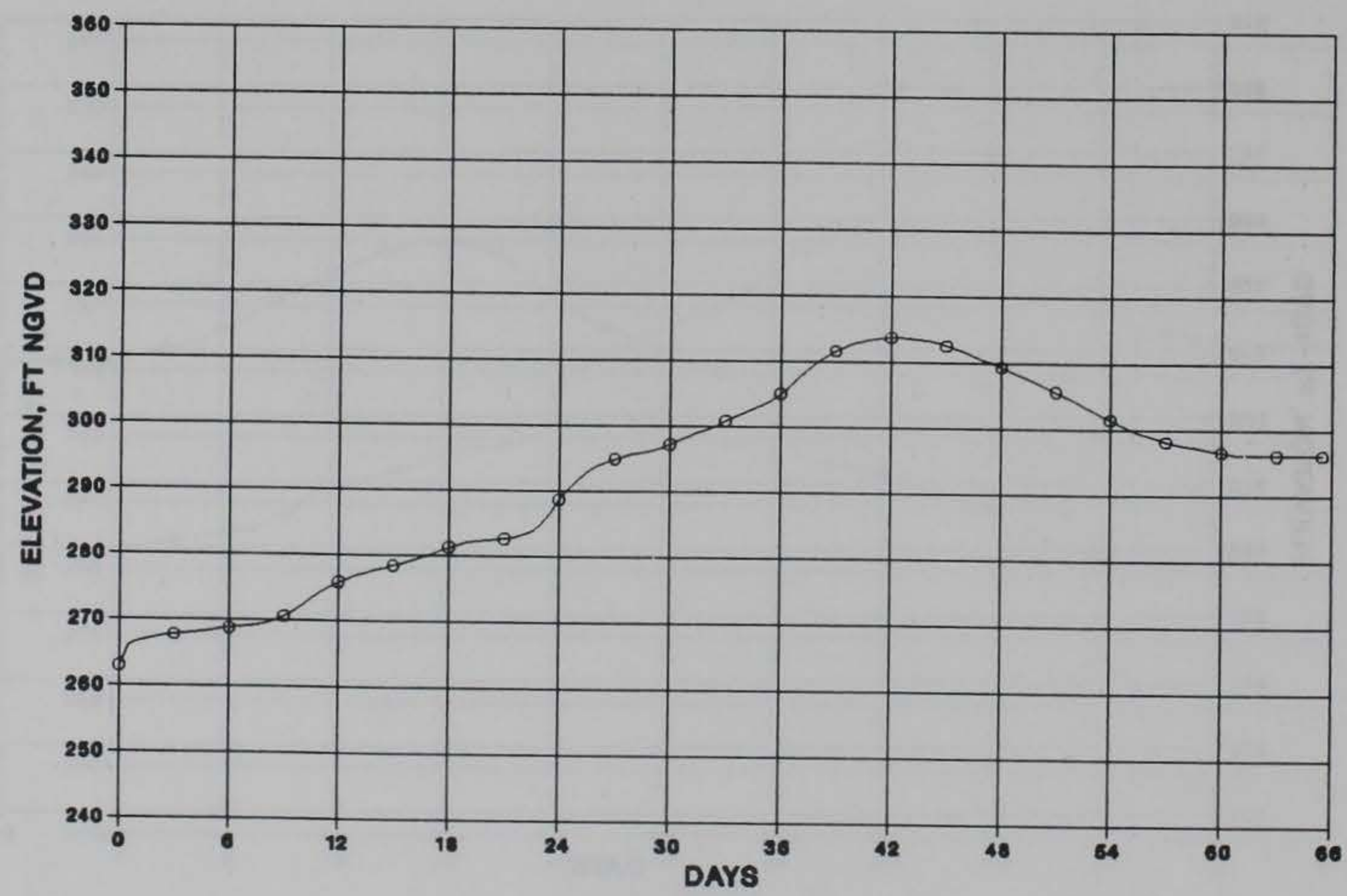

b. Elevation

Figure 70. Results at New Madrid, MO, for Kentucky PMF dam break (gates open) 


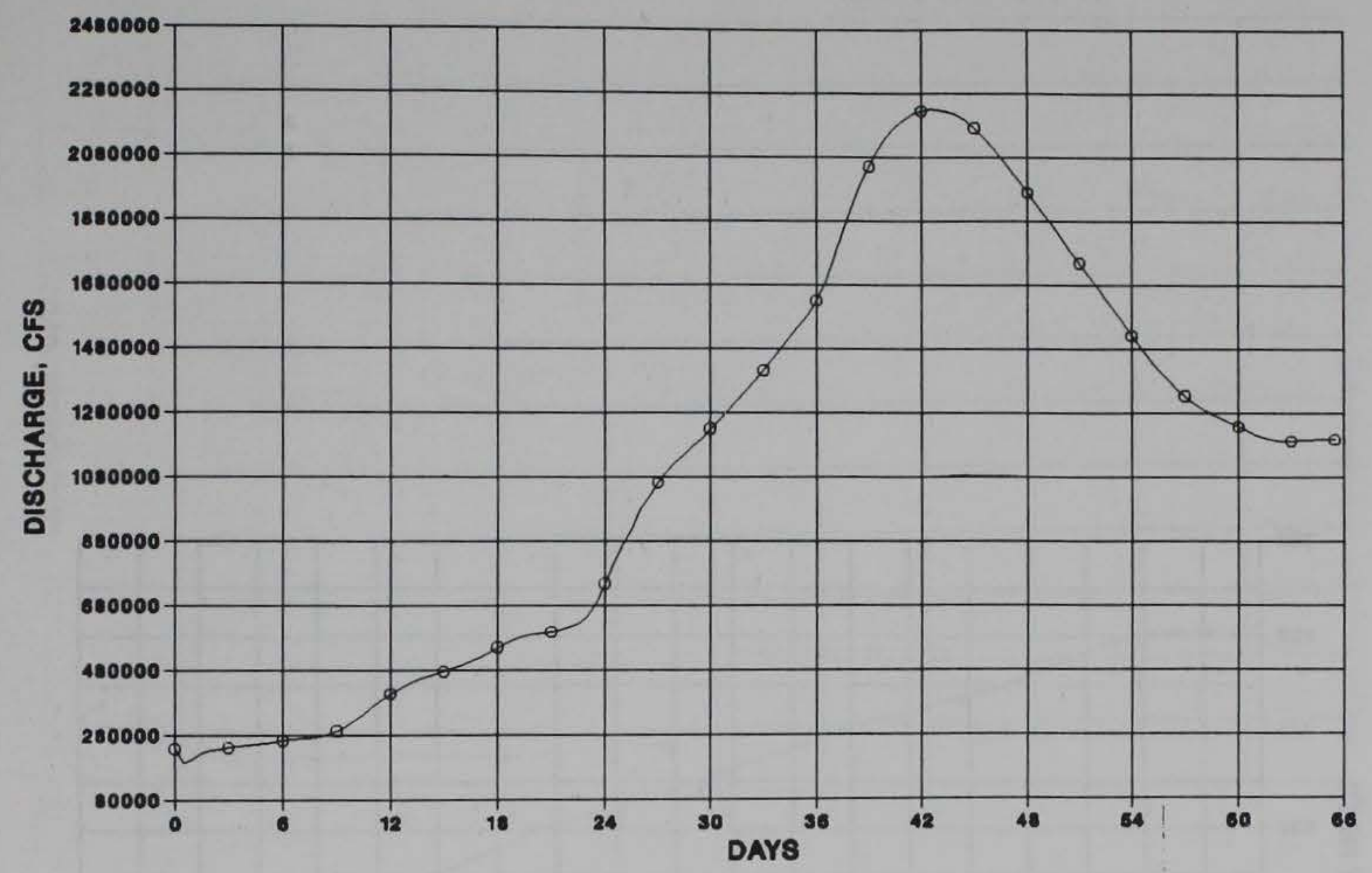

a. Discharge

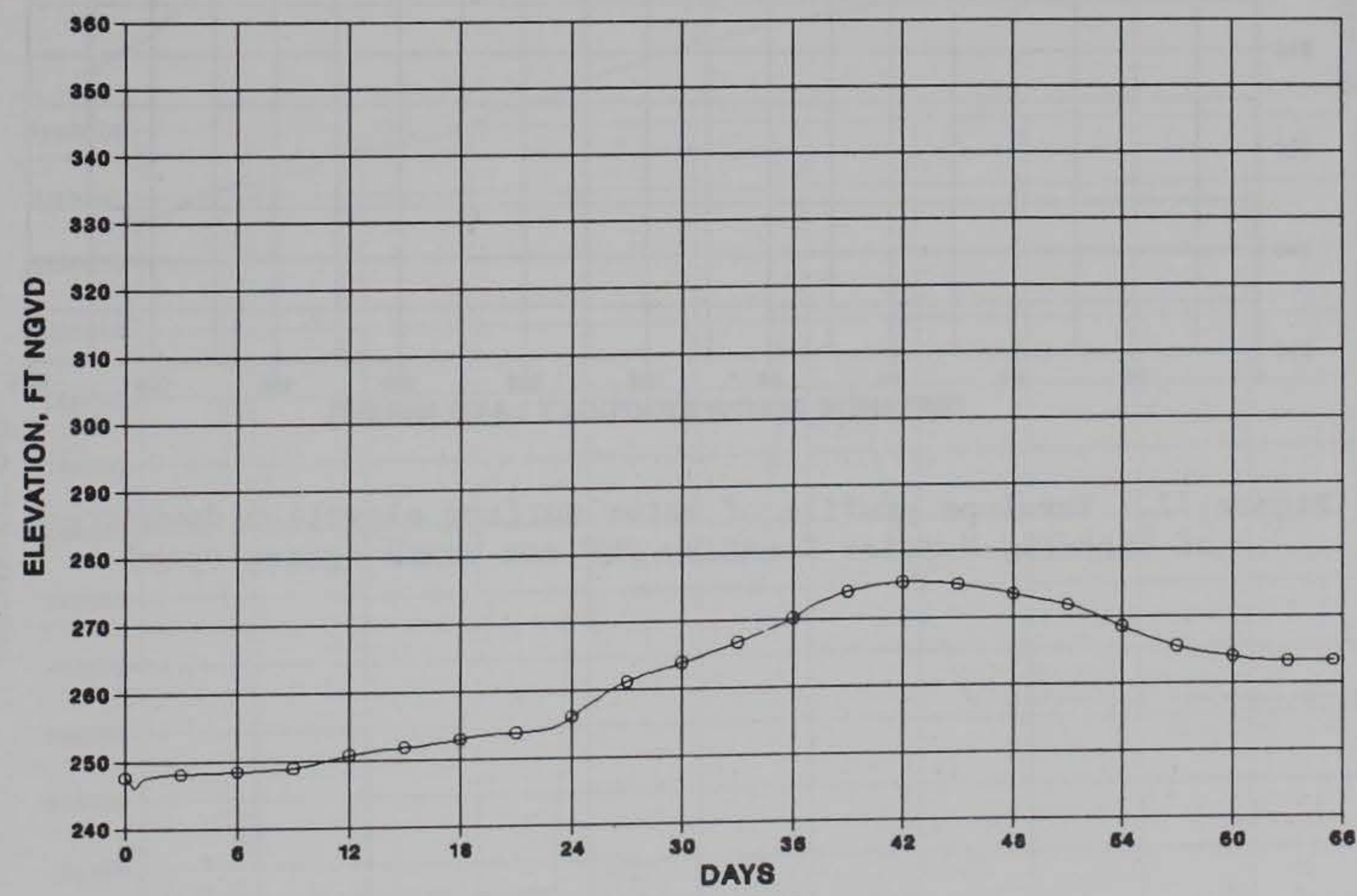

b. Elevation

Figure 71. Results at Caruthersville, MO, for Kentucky PMF dam break (gates open) 


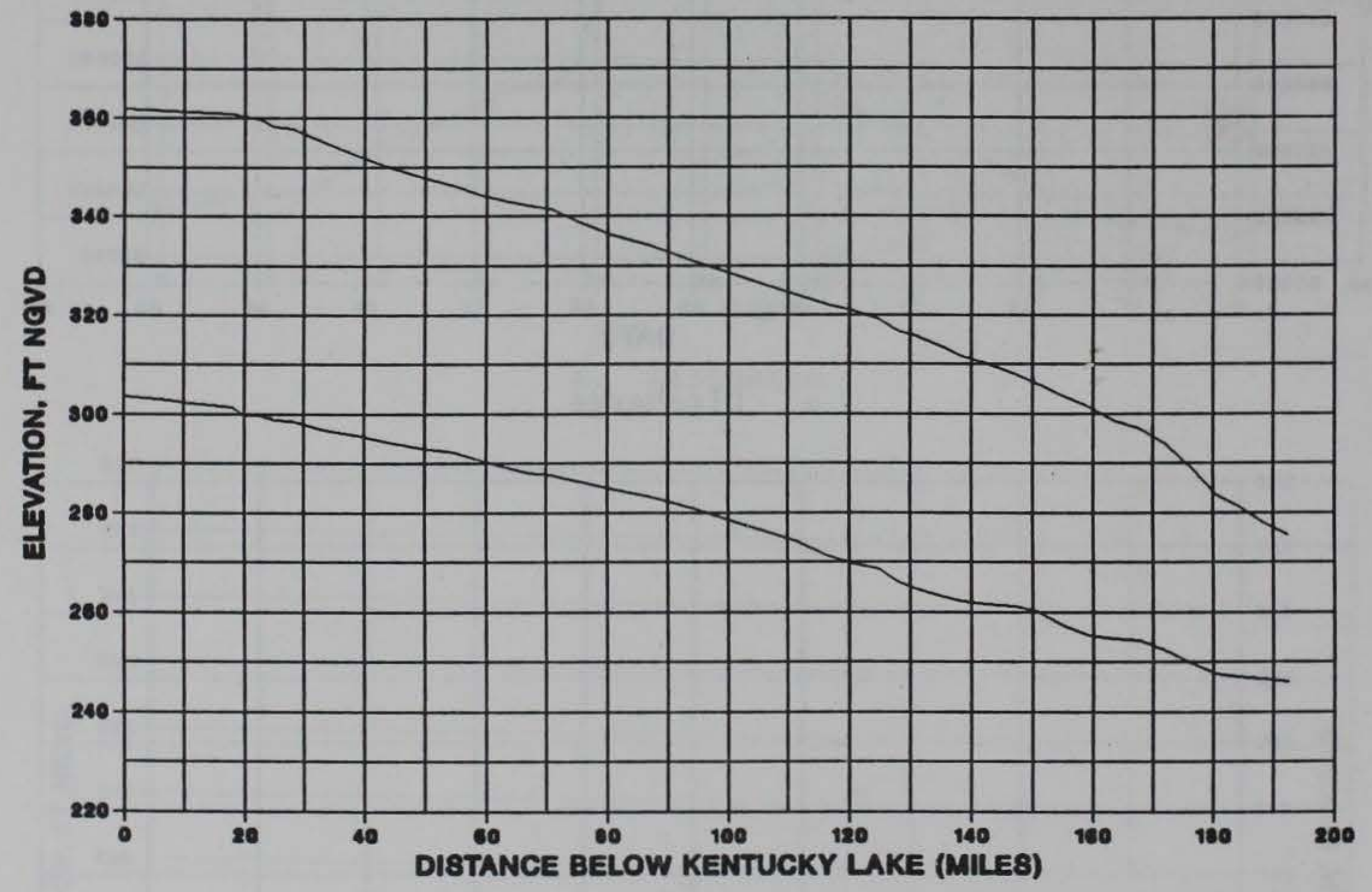

Figure 72. Envelope profile of water surface elevation downstream of Kentucky Dam for Kentucky PMF dam break (gates open) 


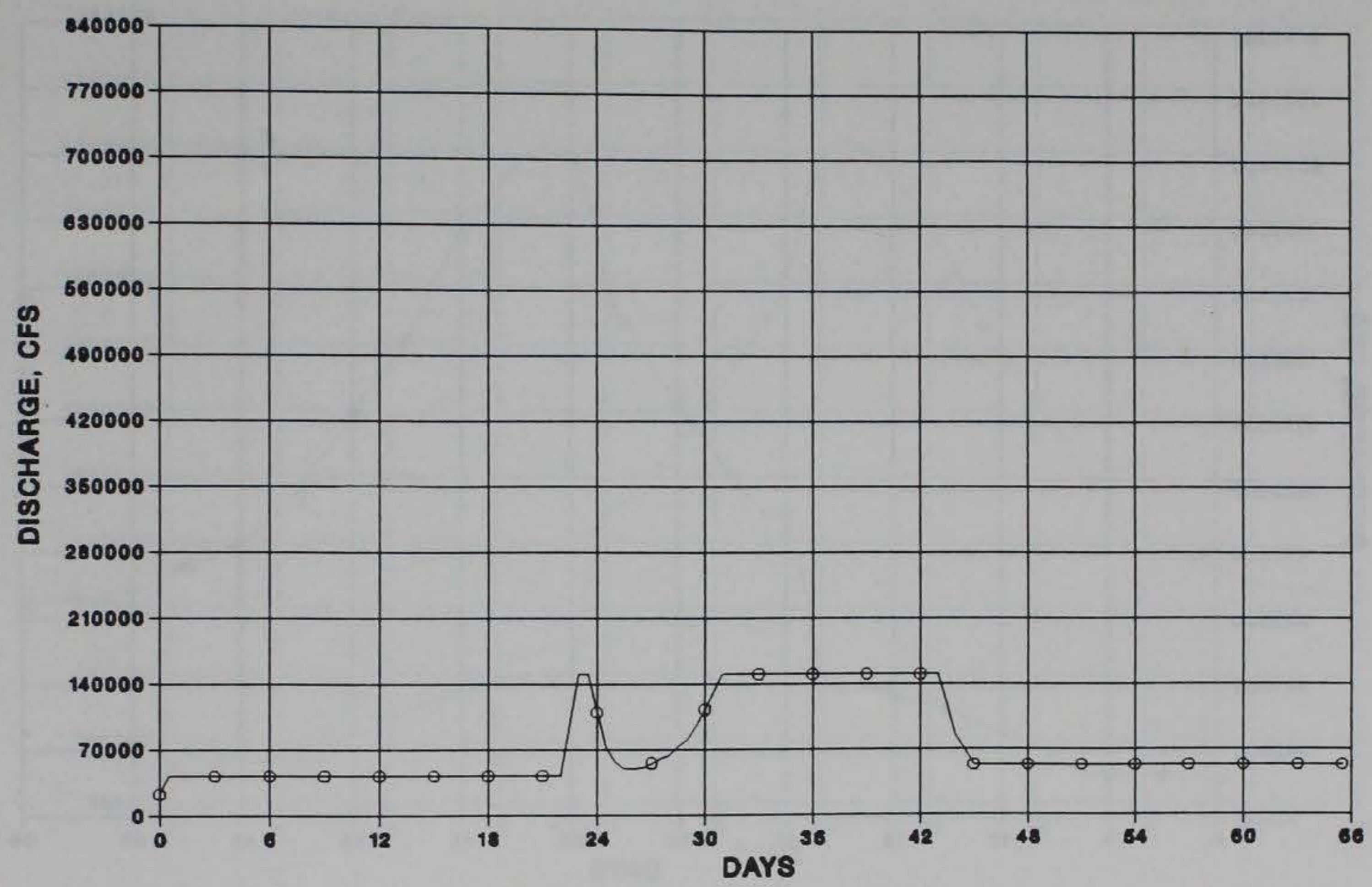

Figure 73. Discharge from Barkley Lake for Kentucky PMF dam break (gates closed)

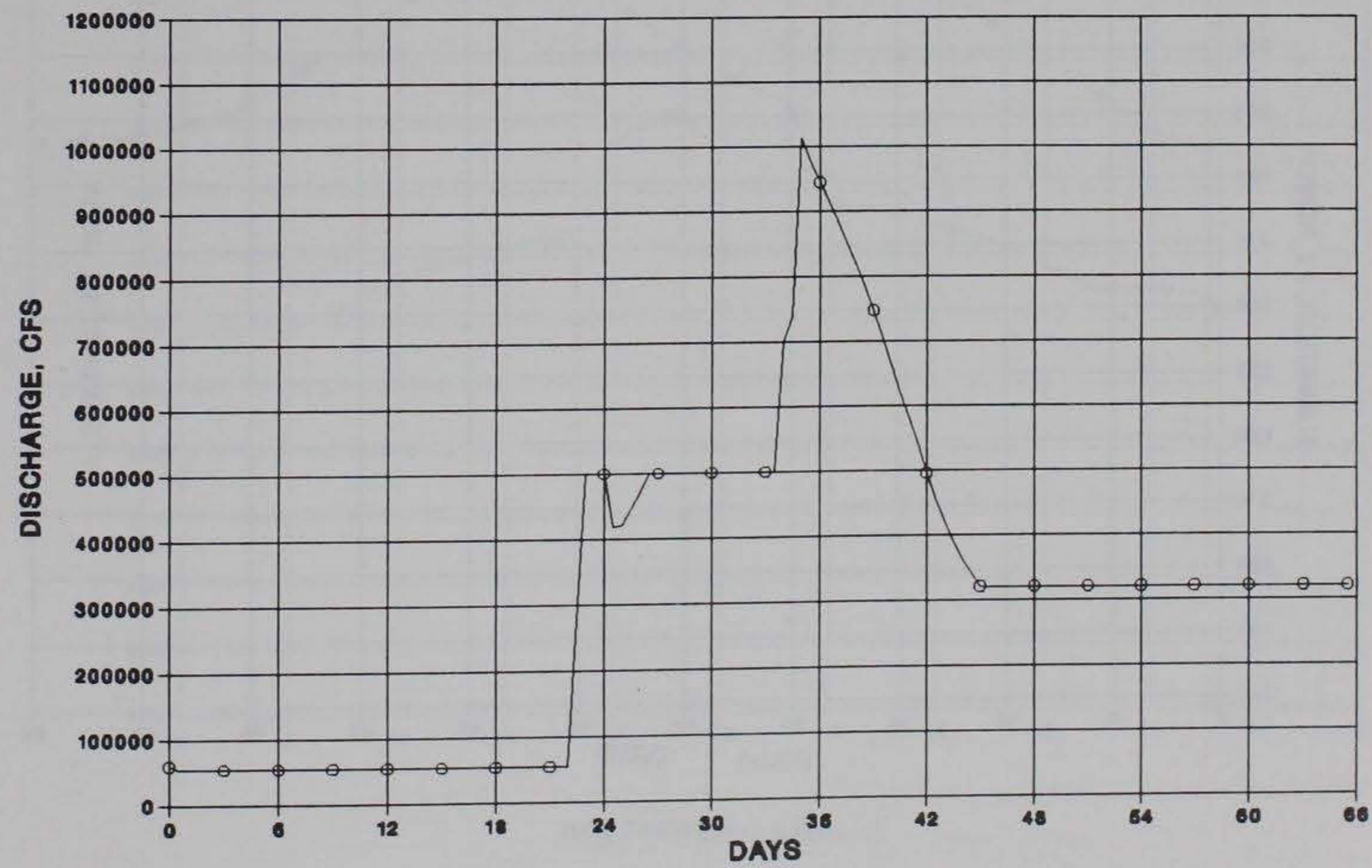

Figure 74. Discharge from Kentucky Lake for Kentucky PMF dam break (gates closed) 


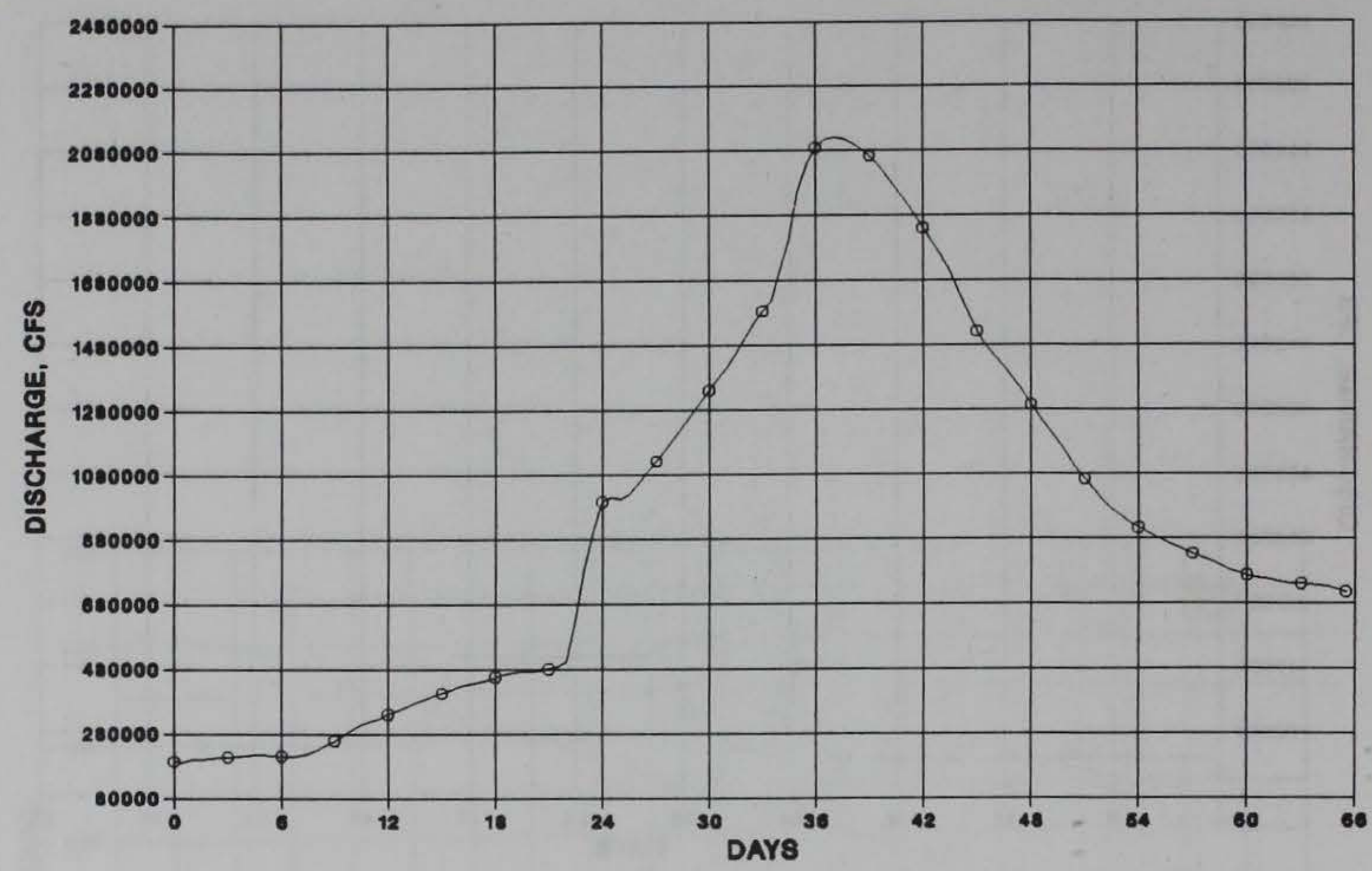

a. Discharge

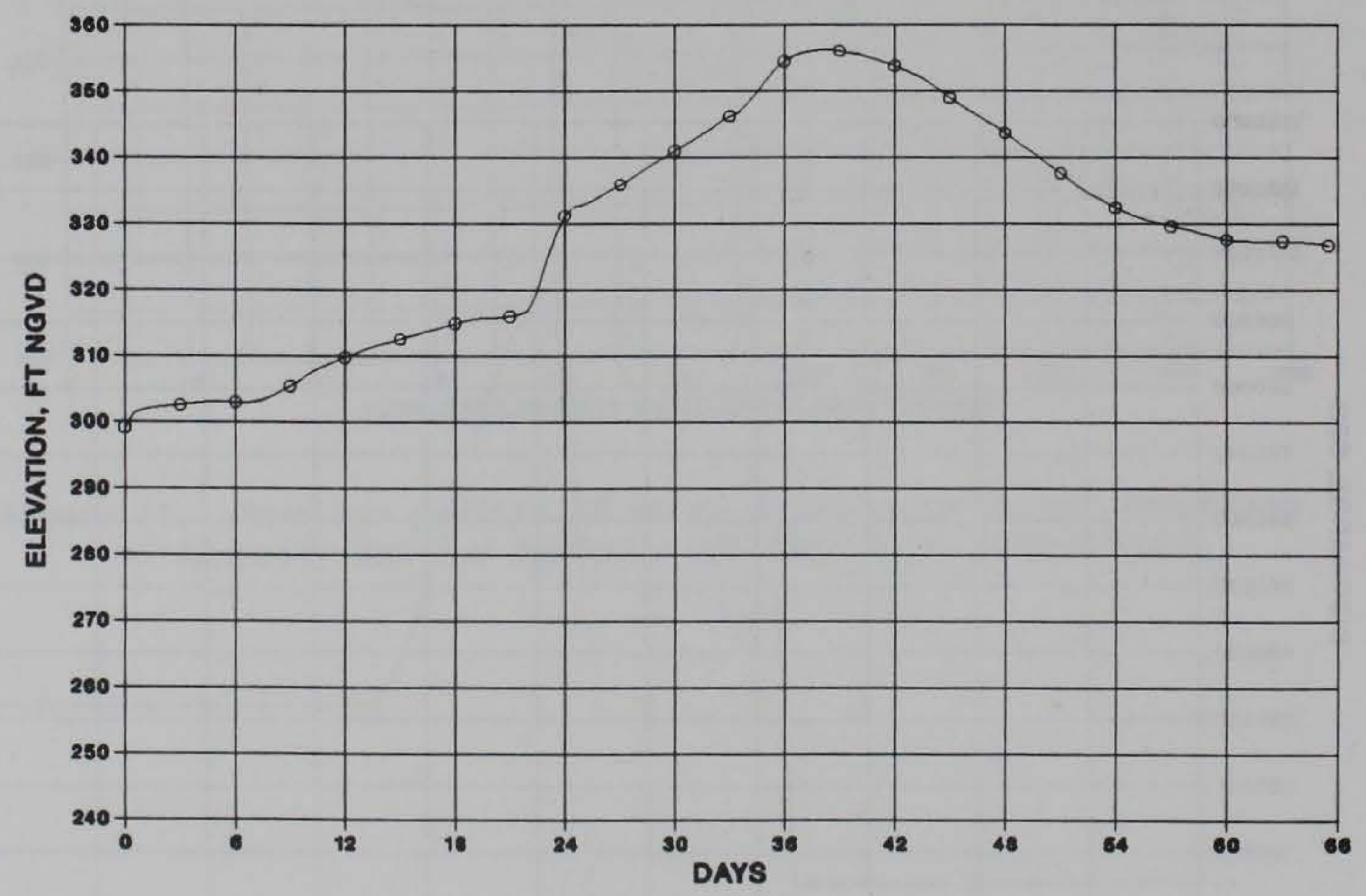

b. Elevation

Figure 75. Results at Paducah, KY, for Kentucky PMF dam break (gates closed) 


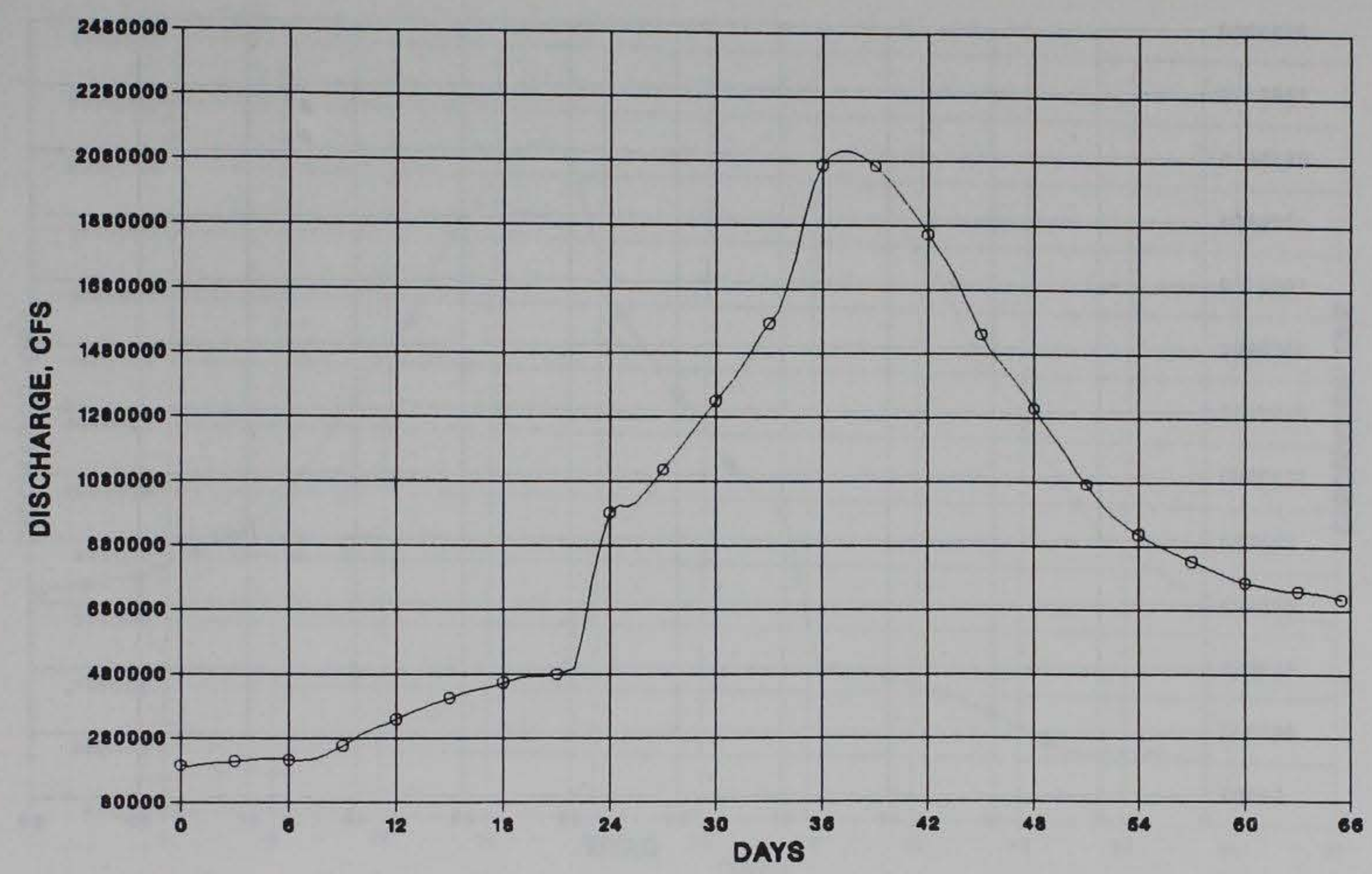

a. Discharge

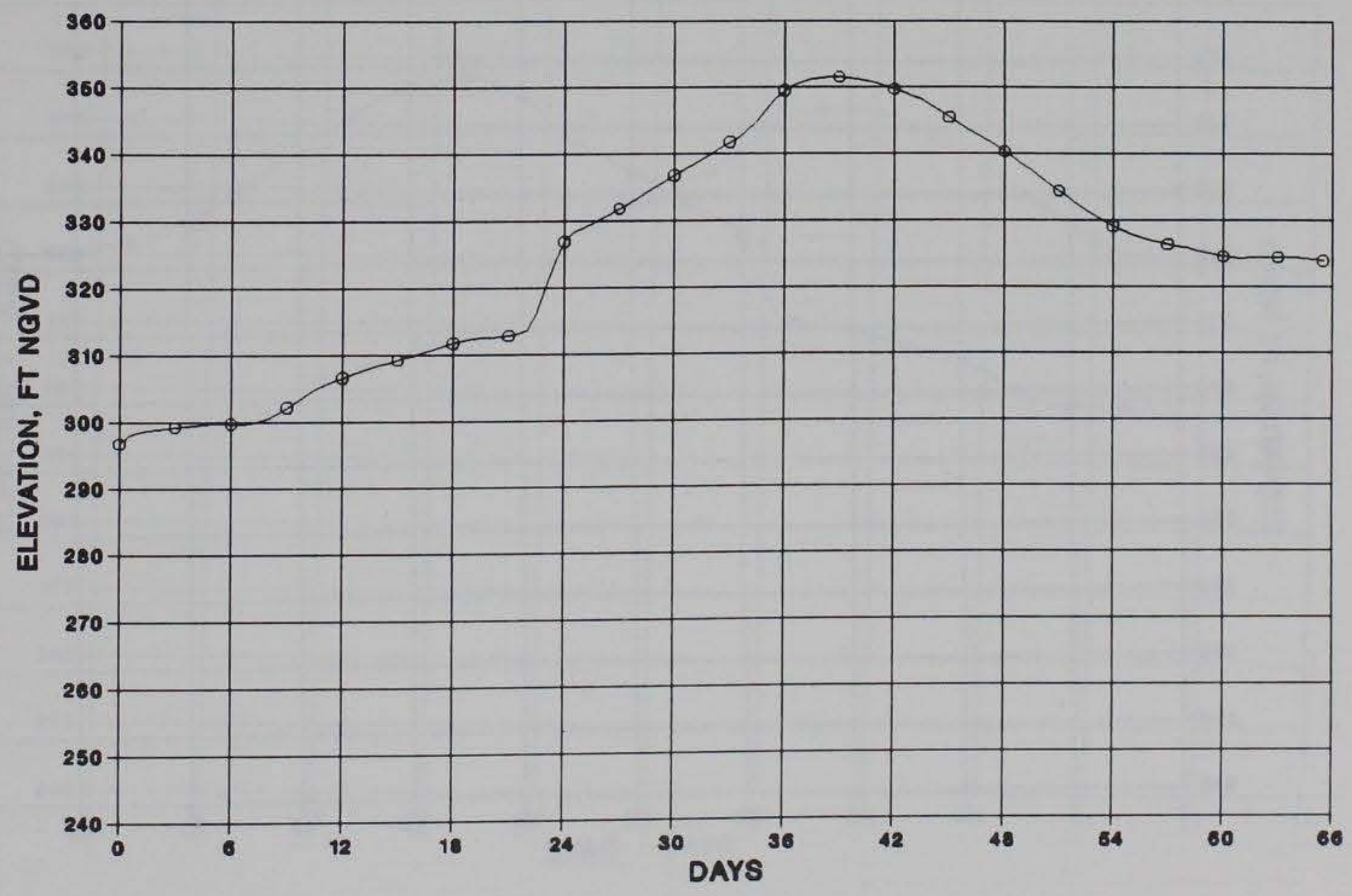

b. Elevation

Figure 76. Results at Metropolis, IL, for Kentucky PMF dam break (gates closed) 


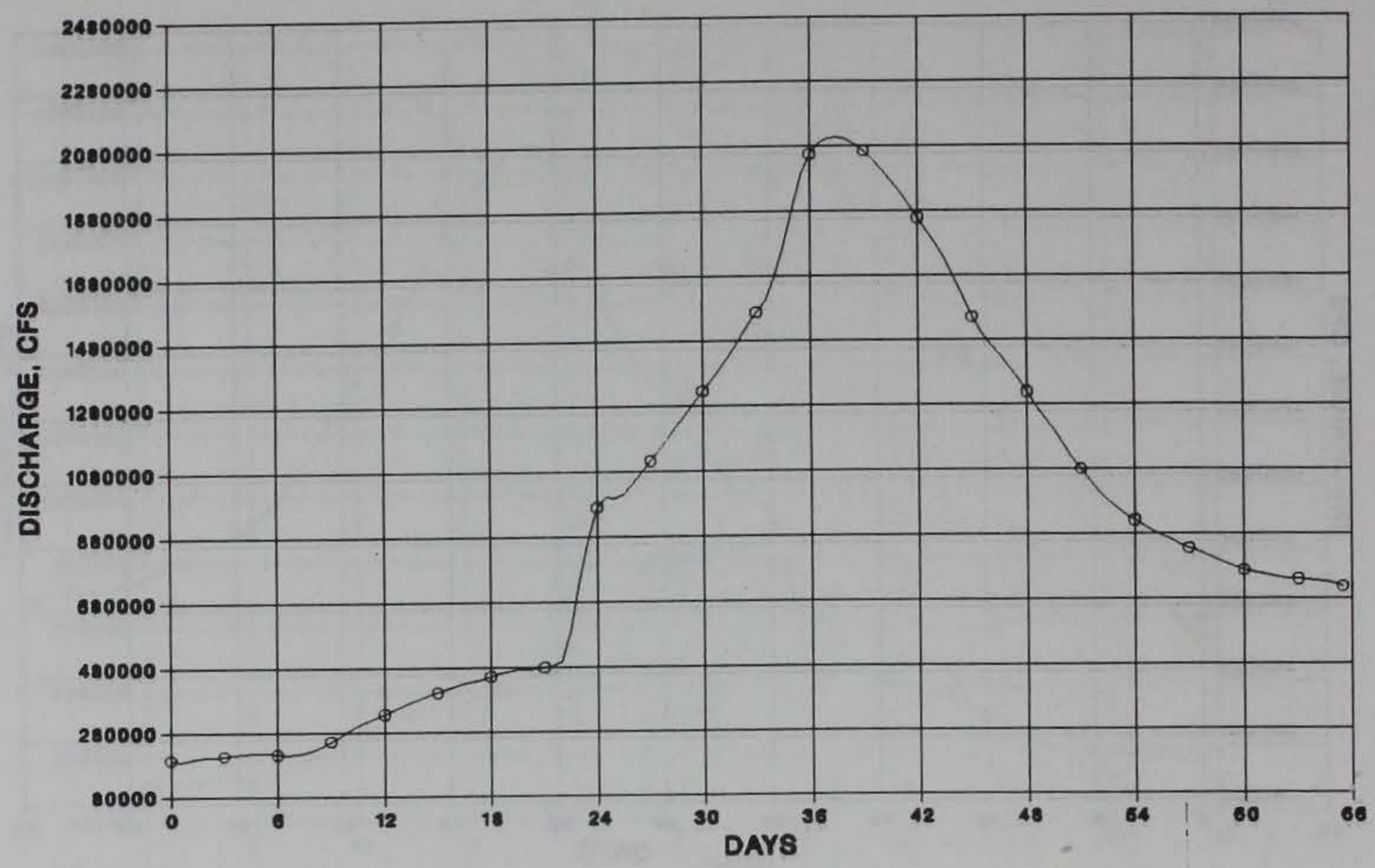

a. Discharge

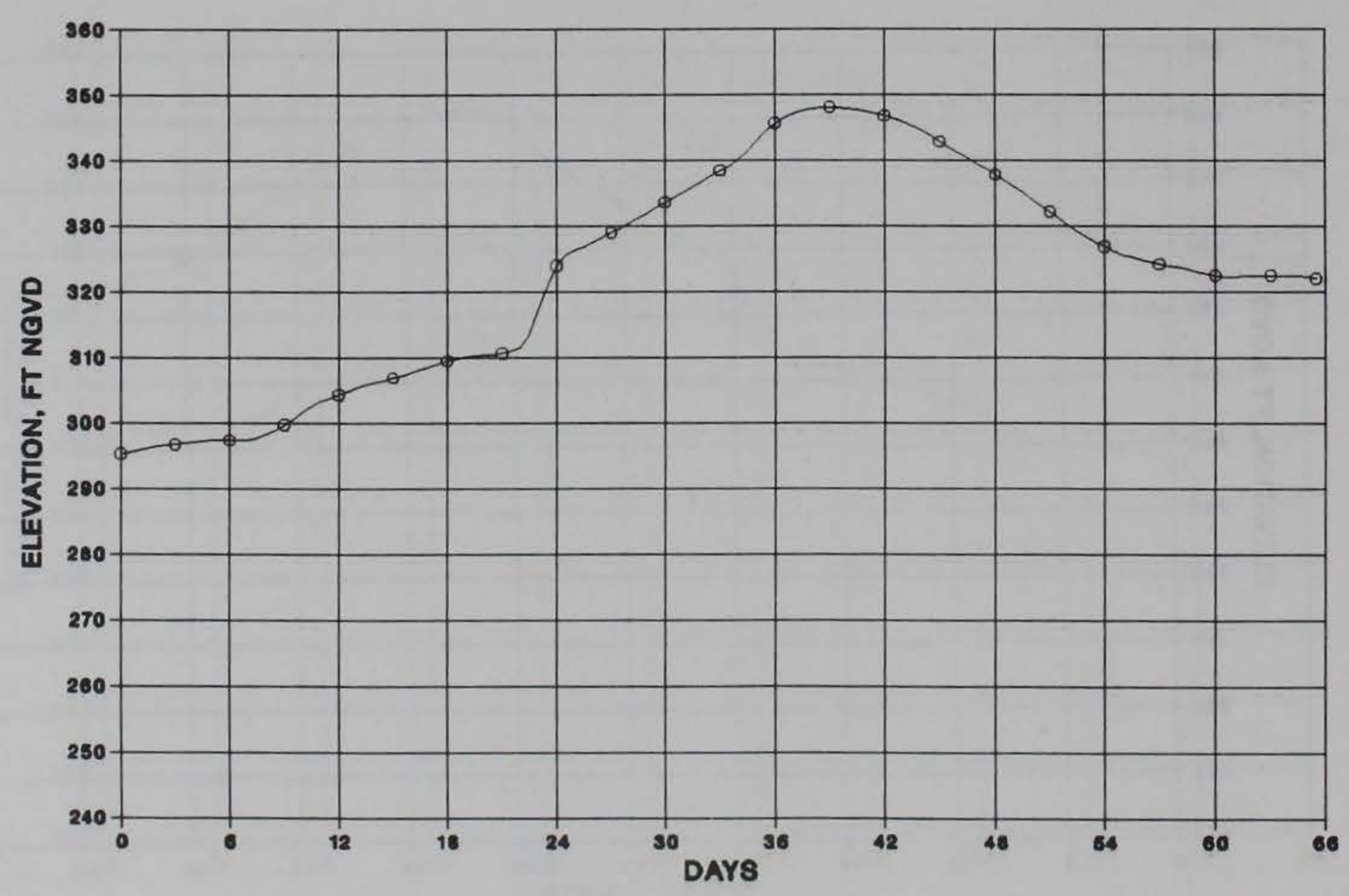

b. Elevation

Figure 77. Results at Joppa, IL, for Kentucky PMF dam break (gates closed) 


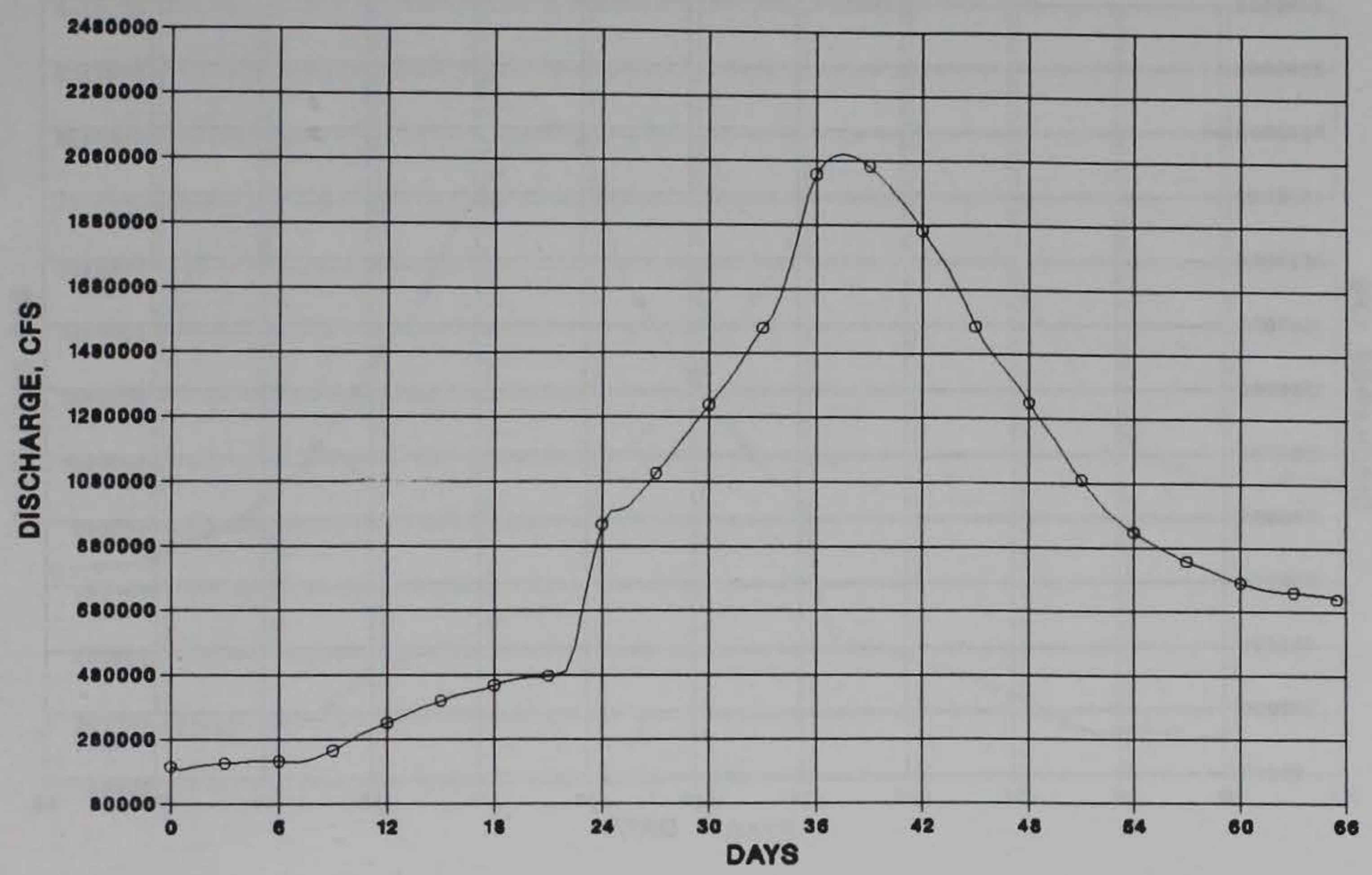

a. Discharge

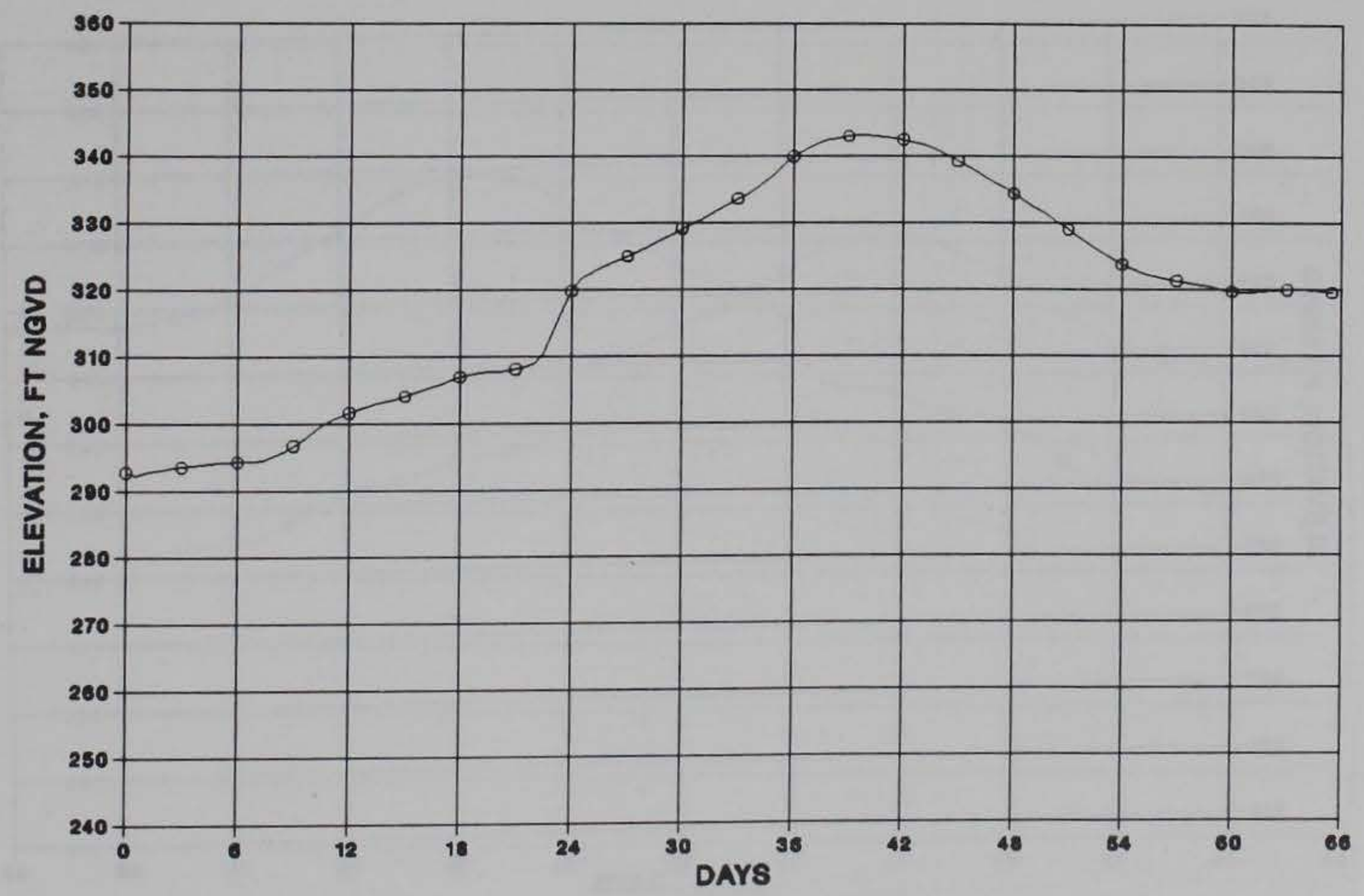

b. Elevation

Figure 78. Results at 01mstead, IL, for Kentucky PMF dam break (gates closed) 


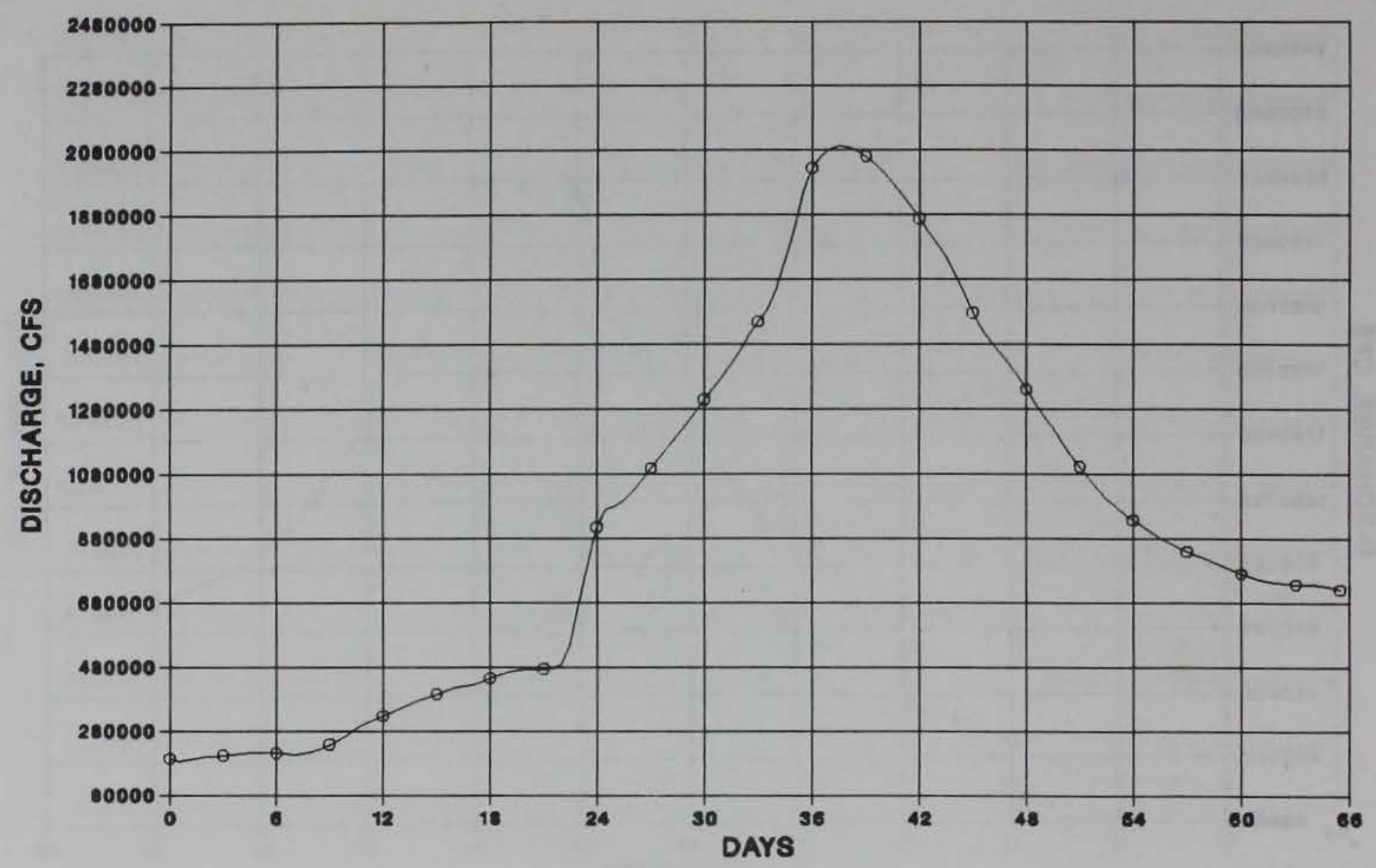

a. Discharge

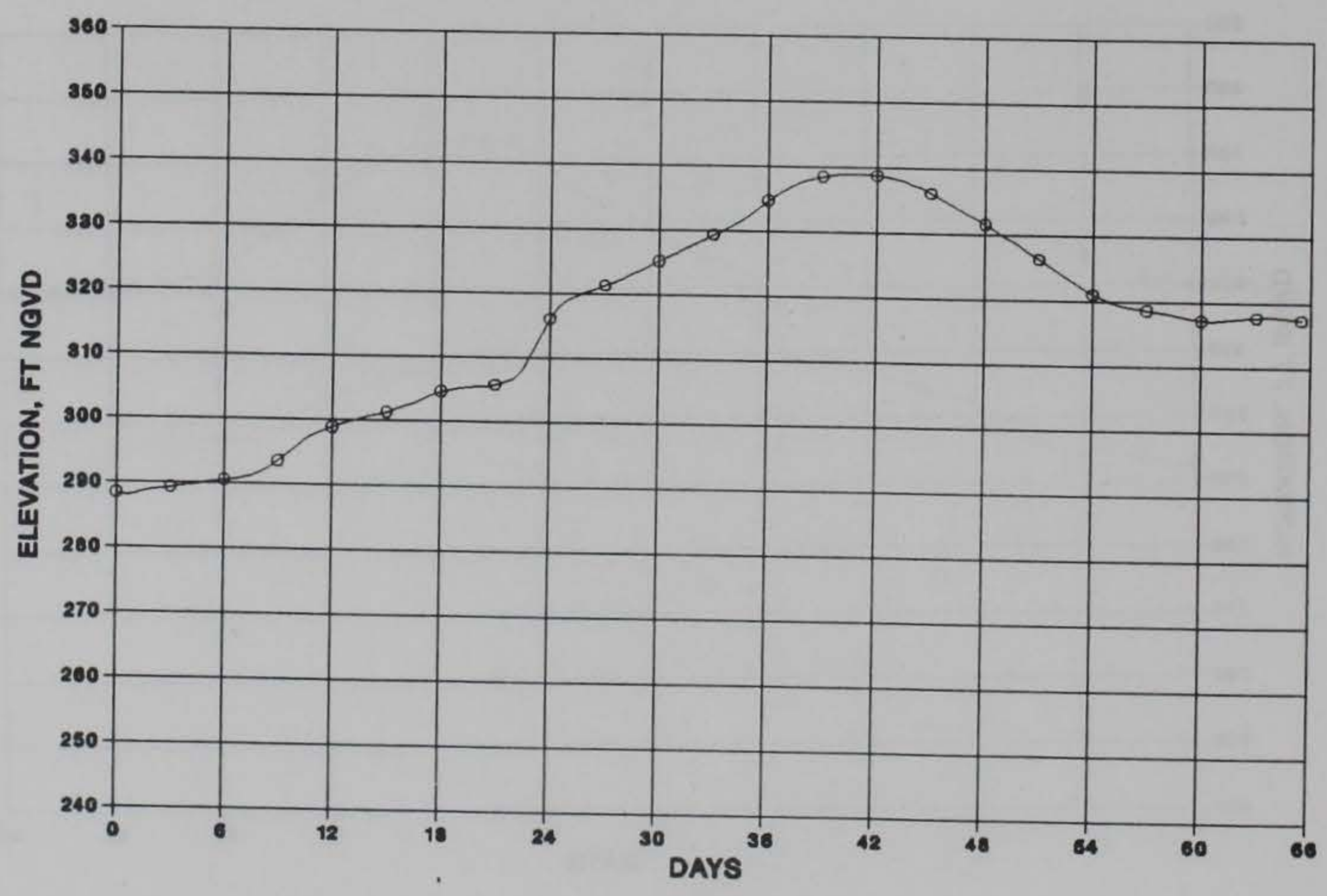

b. Elevation

Figure 79. Results at Cairo, IL, for Kentucky PMF dam break (gates closed) 


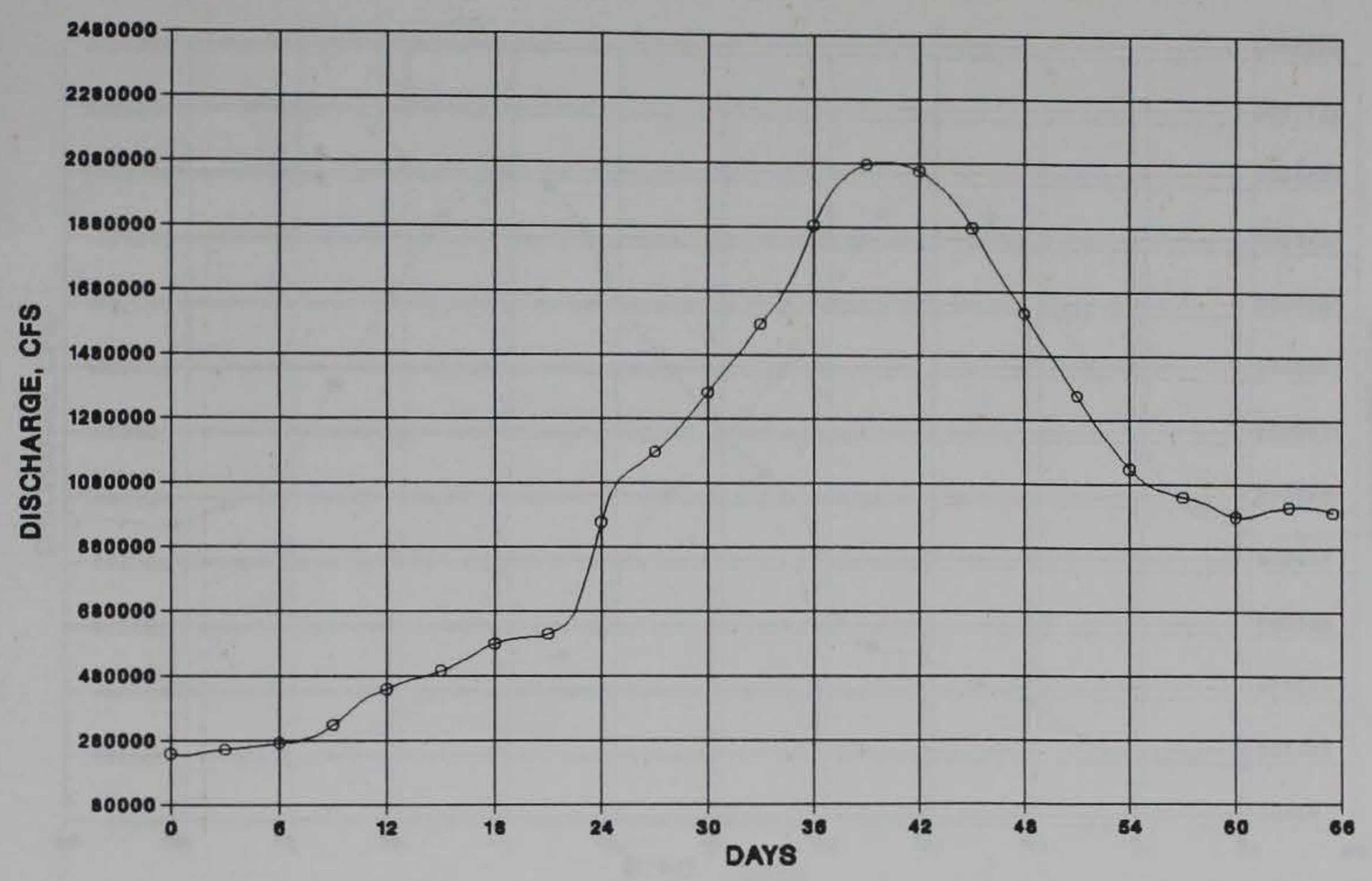

a. Discharge

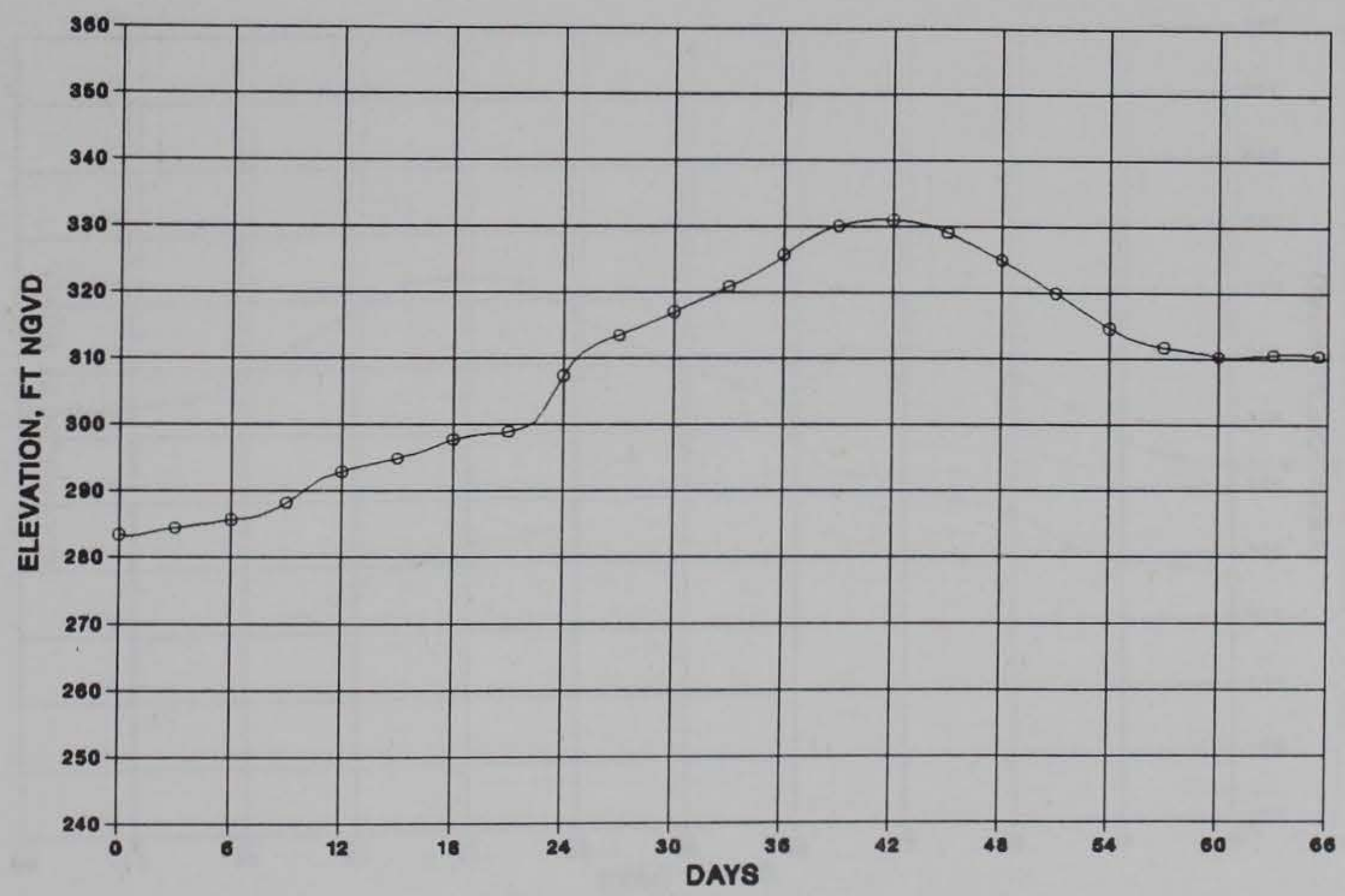

b. Elevation

Figure 80. Results at Columbus, KY, for Kentucky PMF dam break (gates closed) 


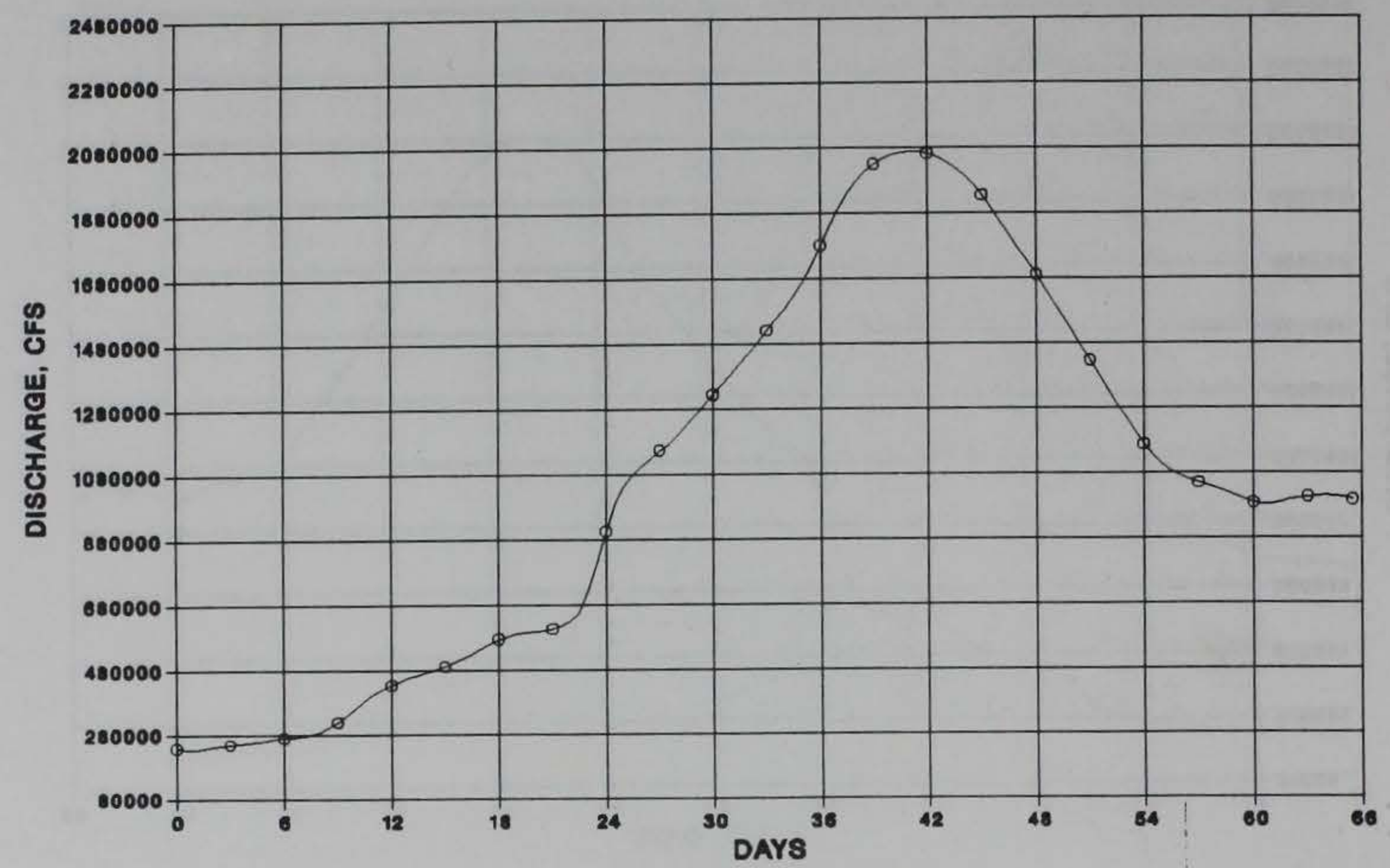

a. Discharge

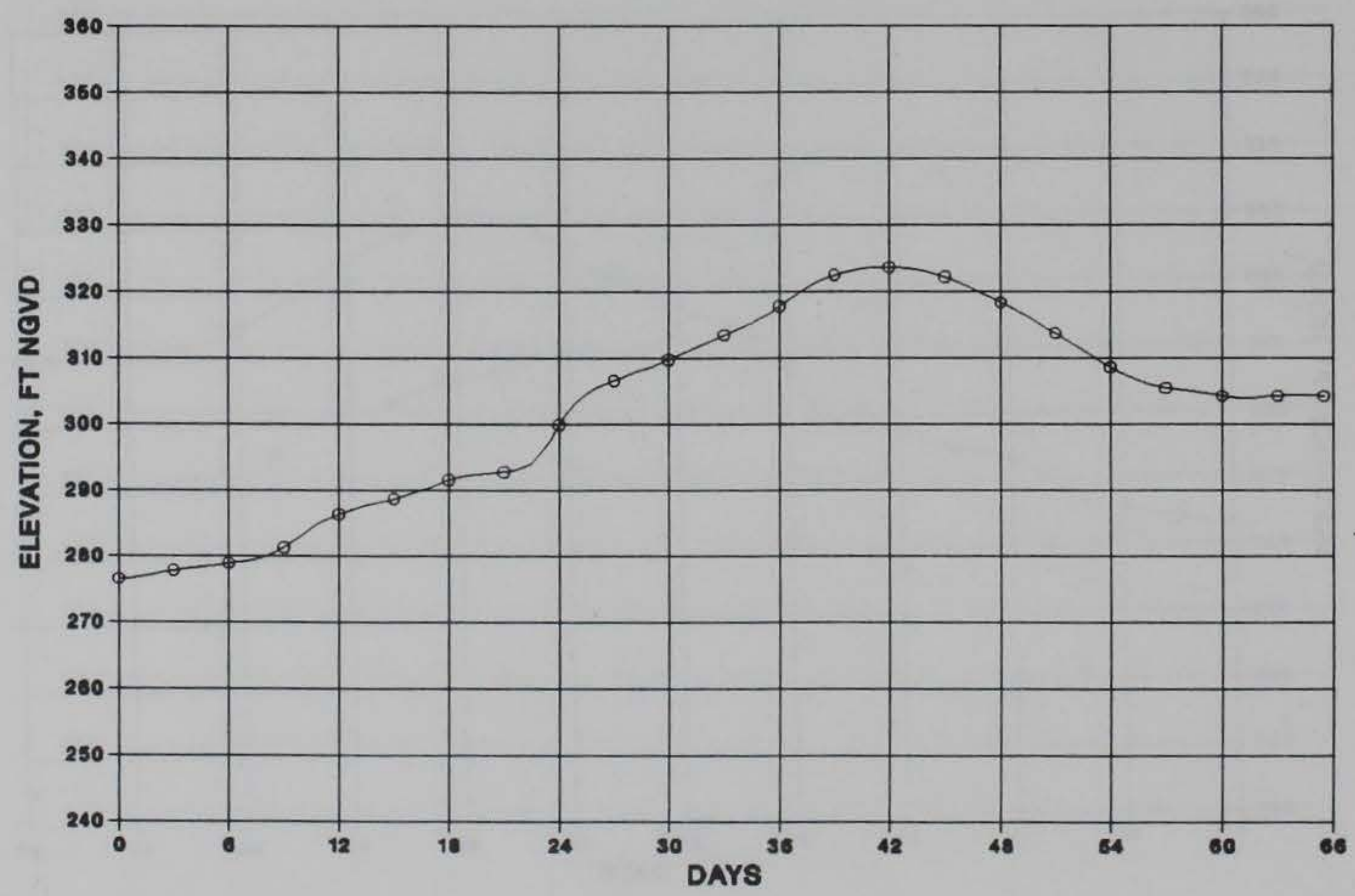

b. Elevation

Figure 81. Results at Hickman, KY, for Kentucky PMF dam break (gates closed) 


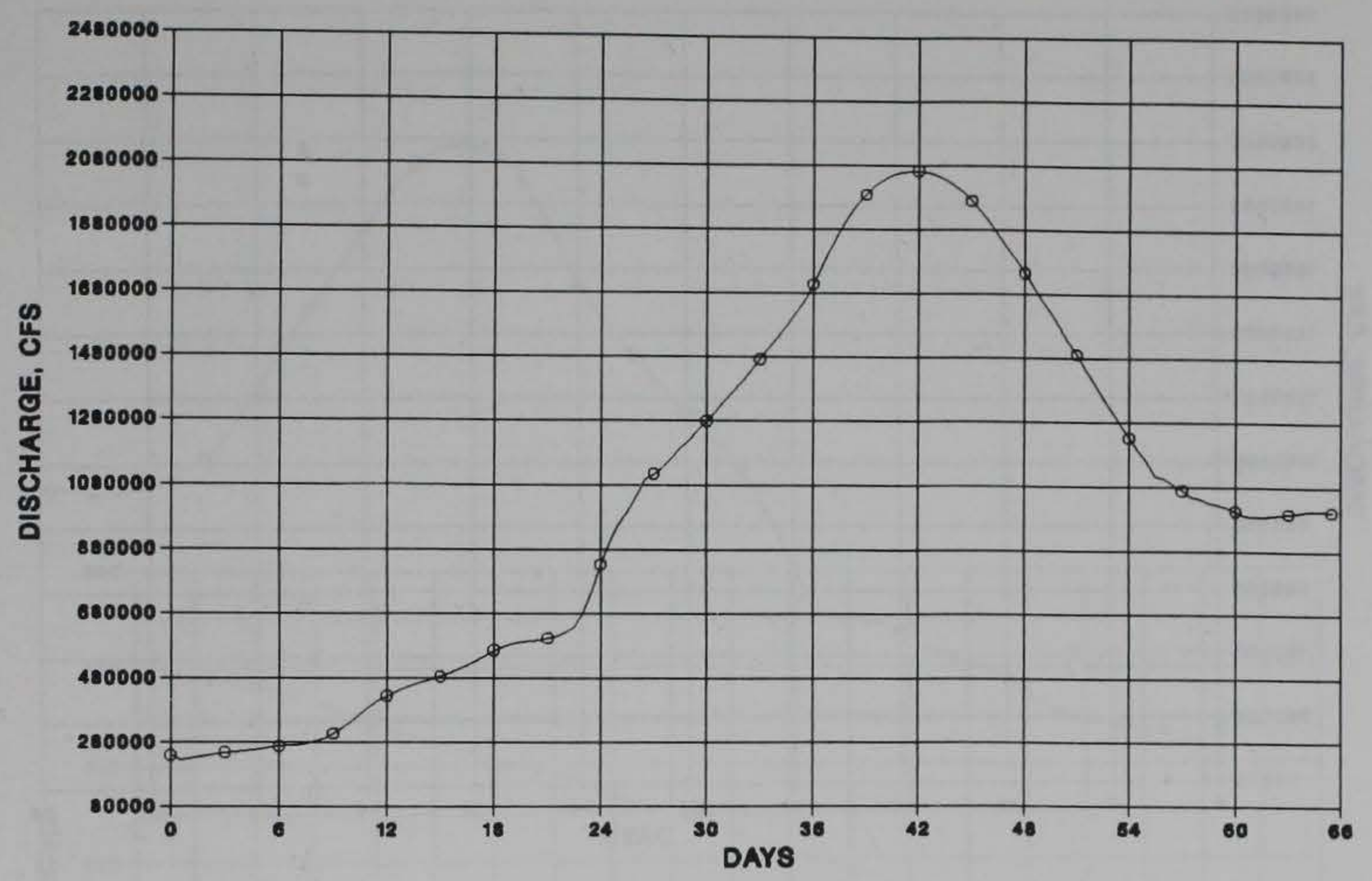

a. Discharge

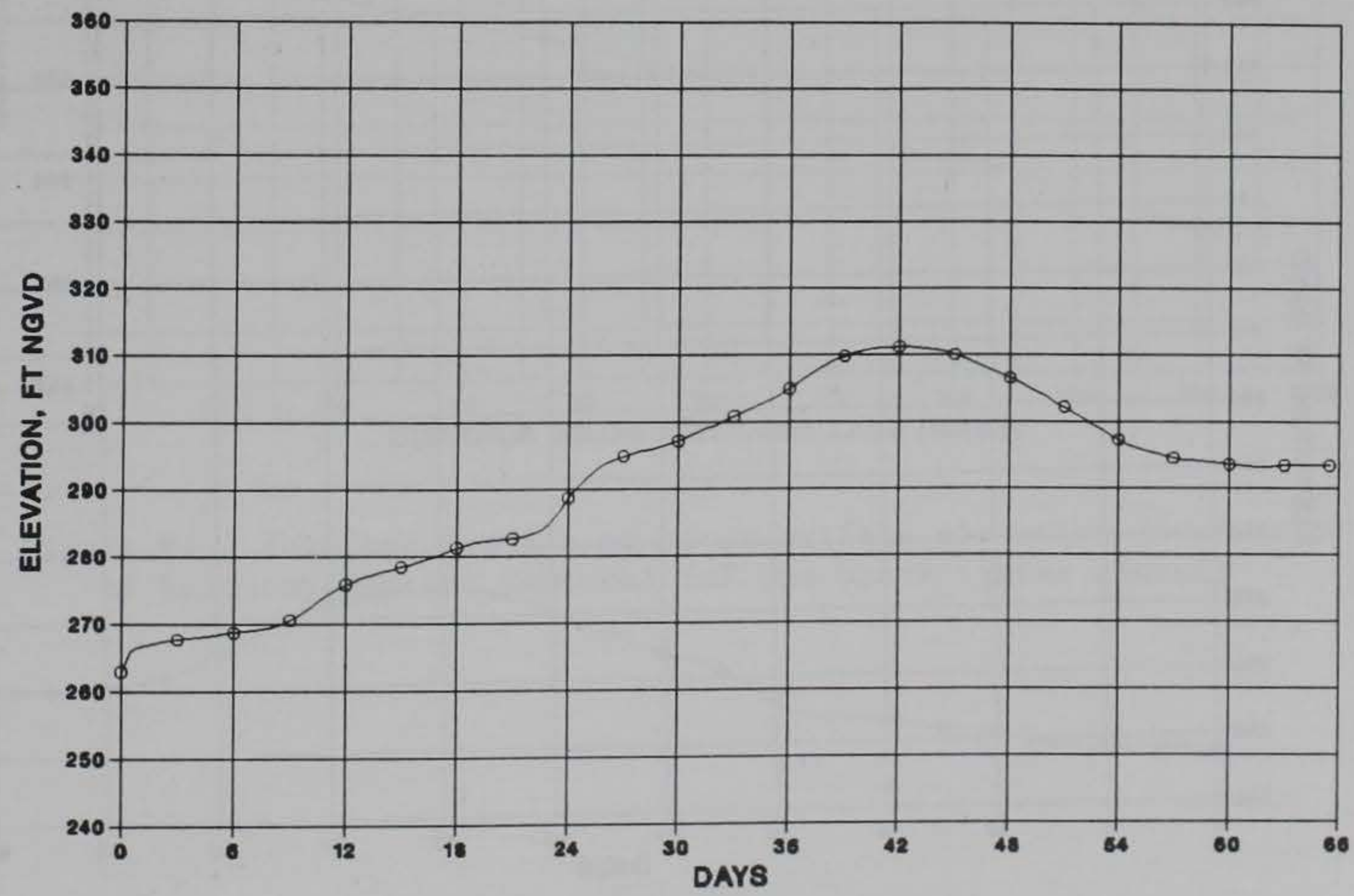

b. Elevation

Figure 82. Results at New Madrid, MO, for Kentucky PMF dam break (gates closed) 


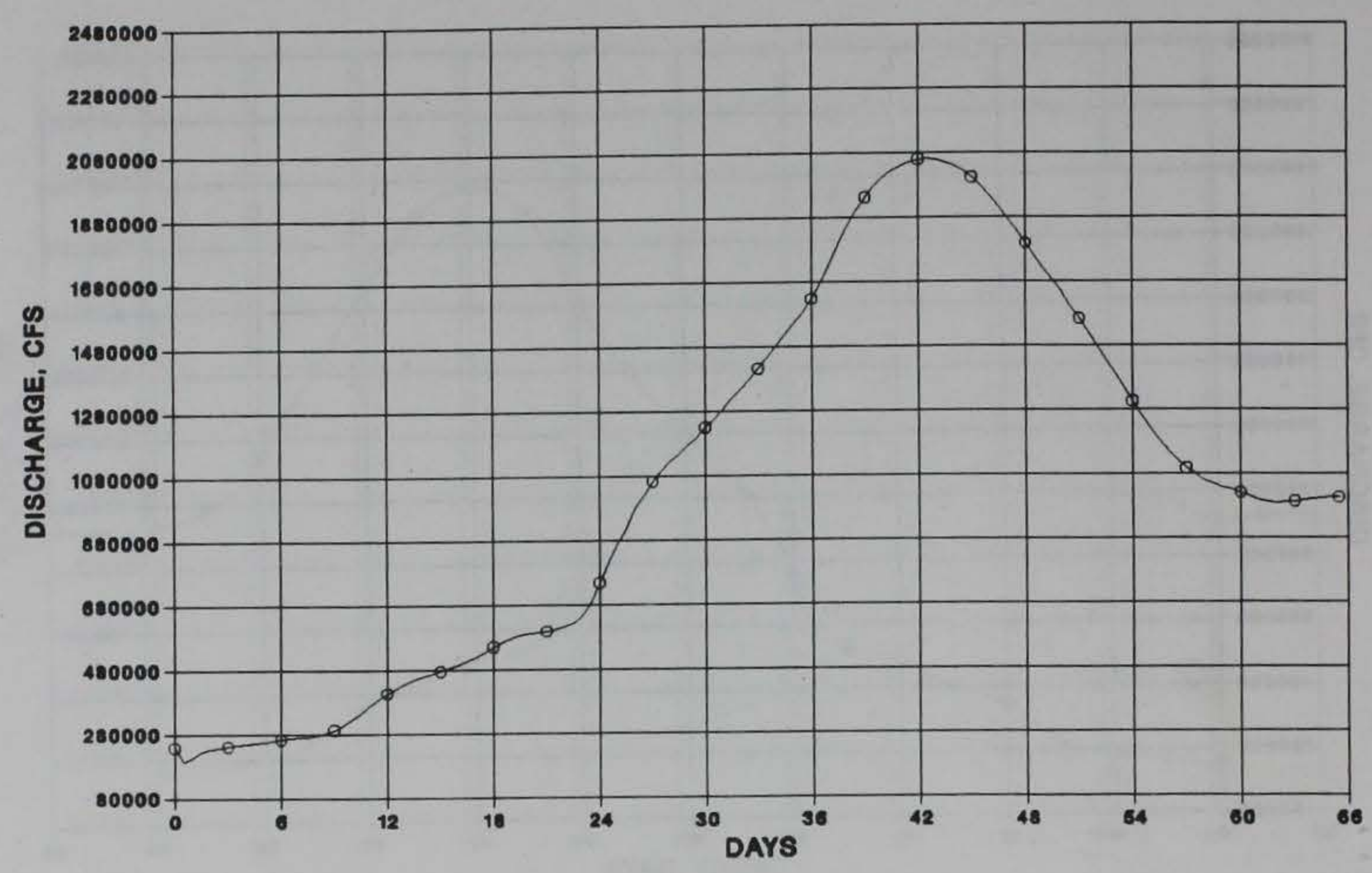

a. Discharge

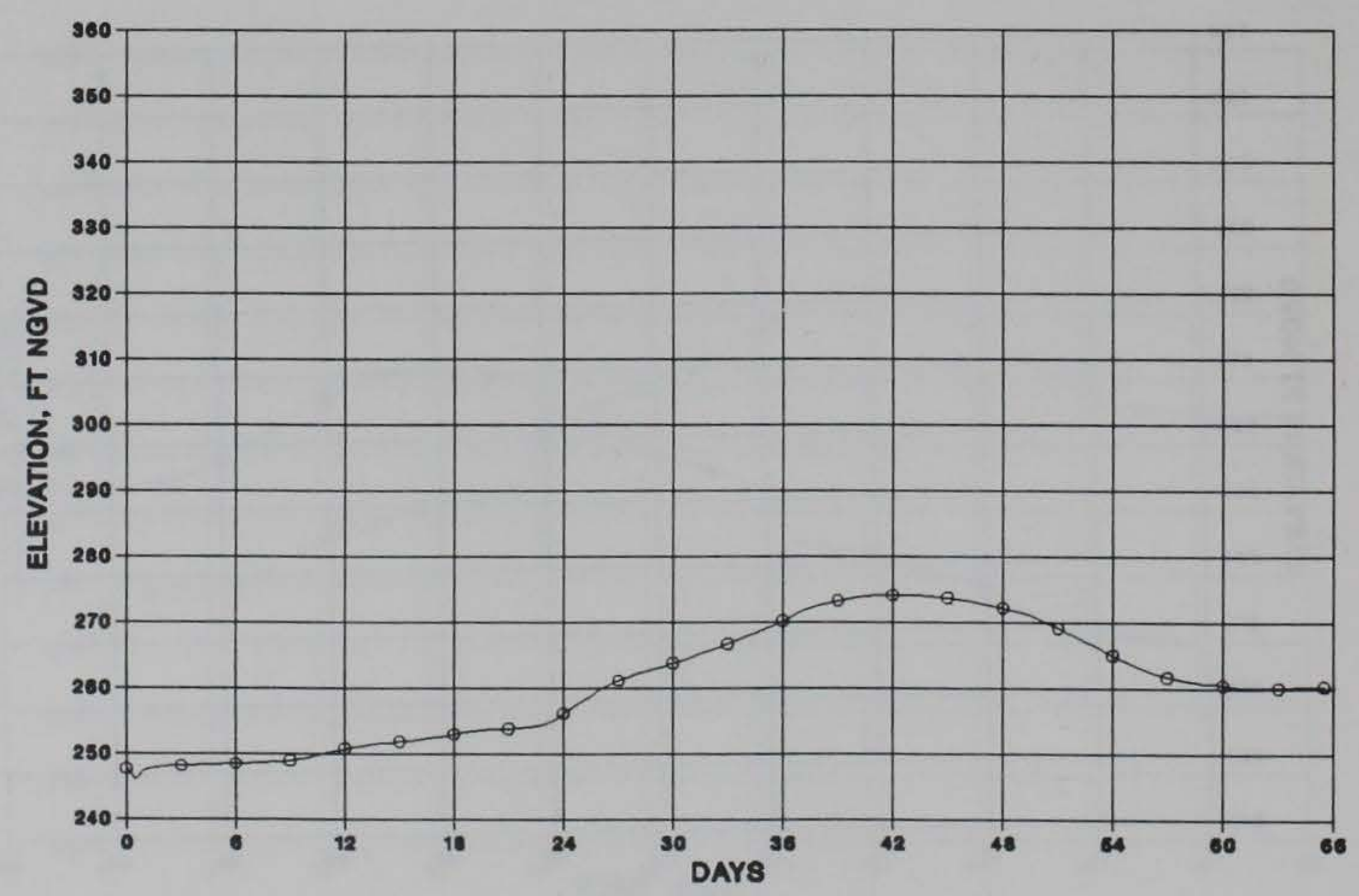

b. Elevation

Figure 83. Results at Caruthersville, MO, for Kentucky PMF dam break (gates closed) 


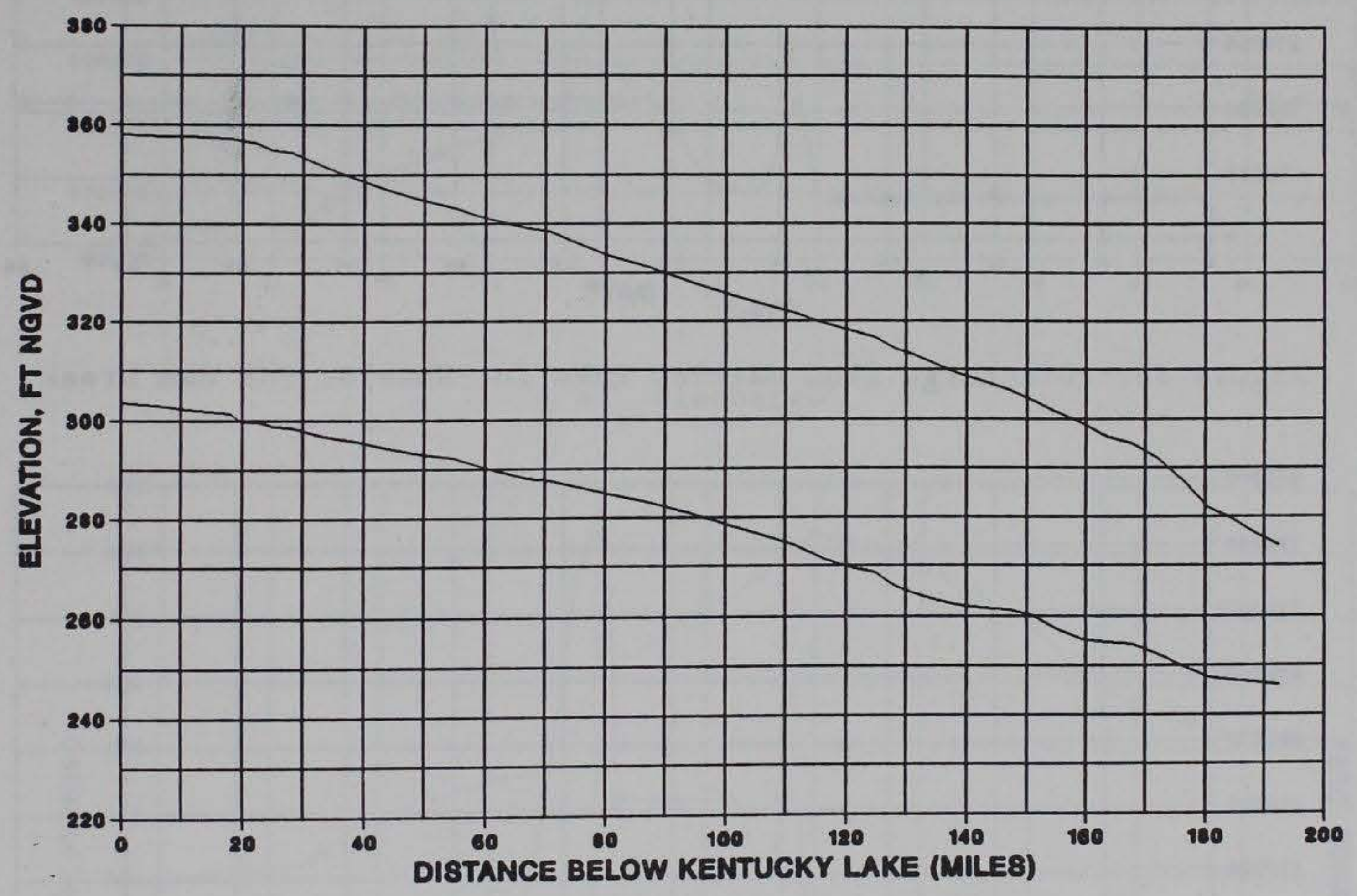

Figure 84. Envelope profile of water surface elevation downstream of Kentucky Dam for Kentucky PMF dam break (gates closed) 


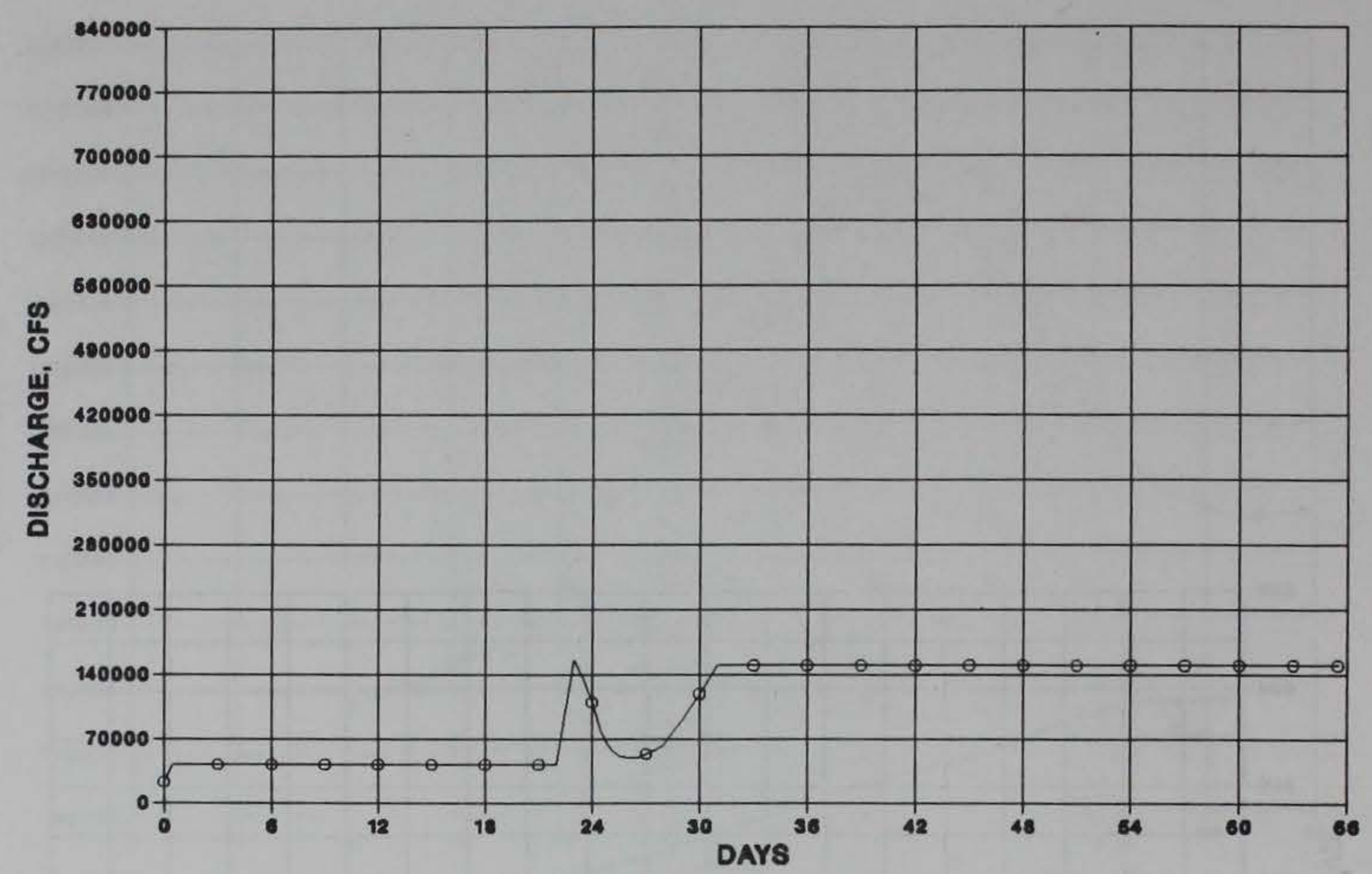

Figure 85. Discharge from Barkley Lake for Wheeler PMF dam break

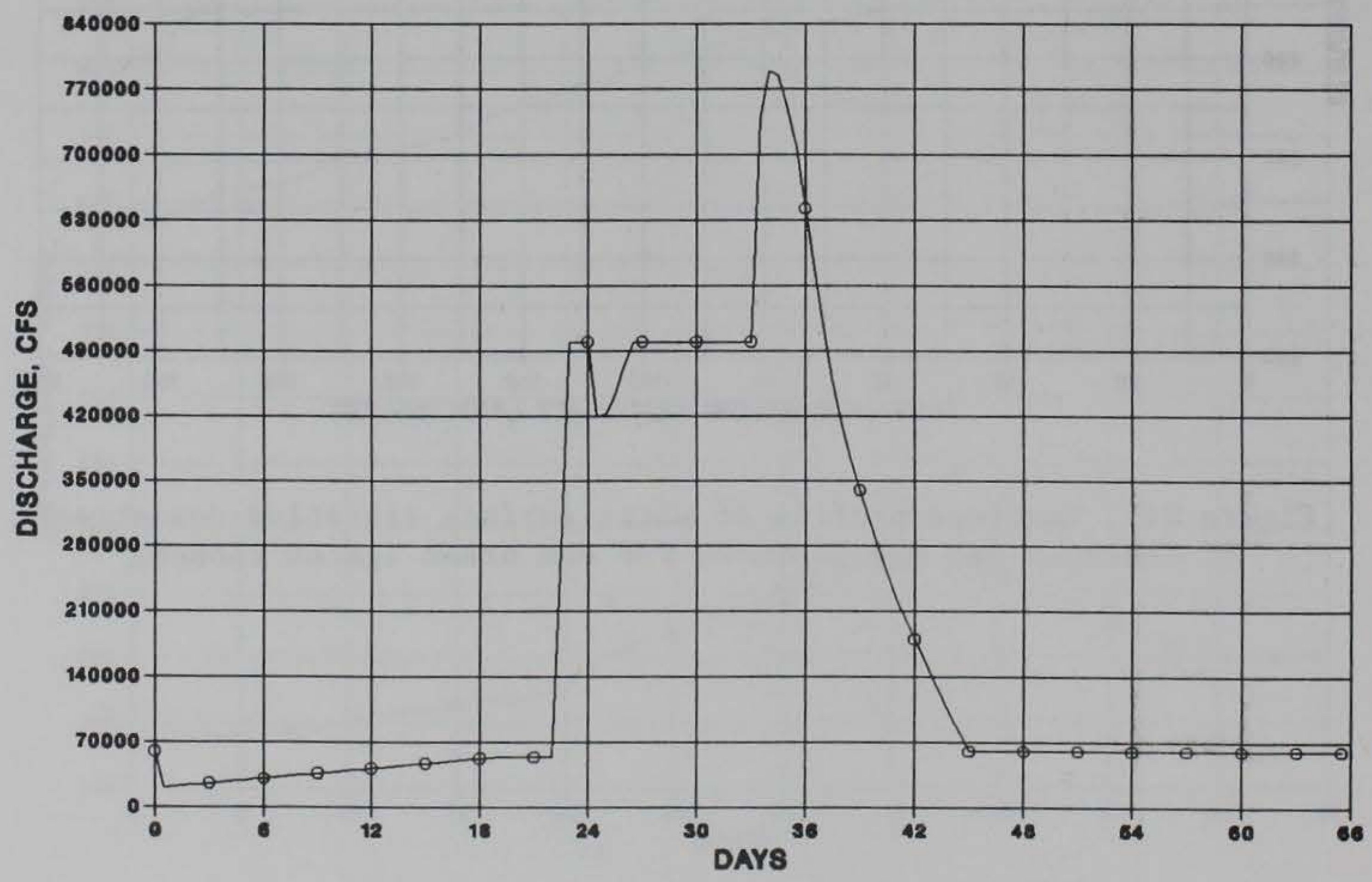

Figure 86. Discharge from Kentucky Lake for Wheeler PMF dam break 


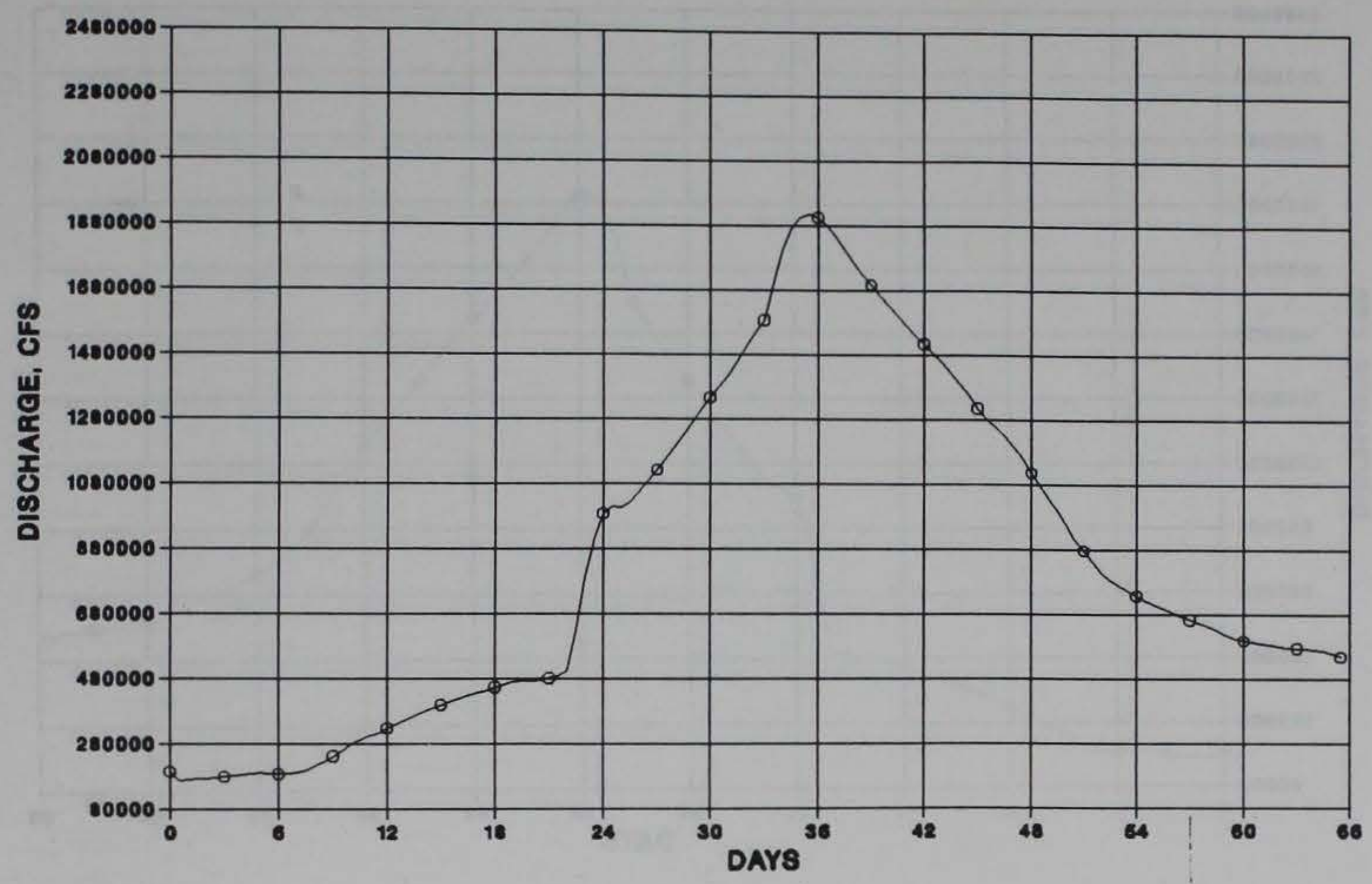

a. Discharge

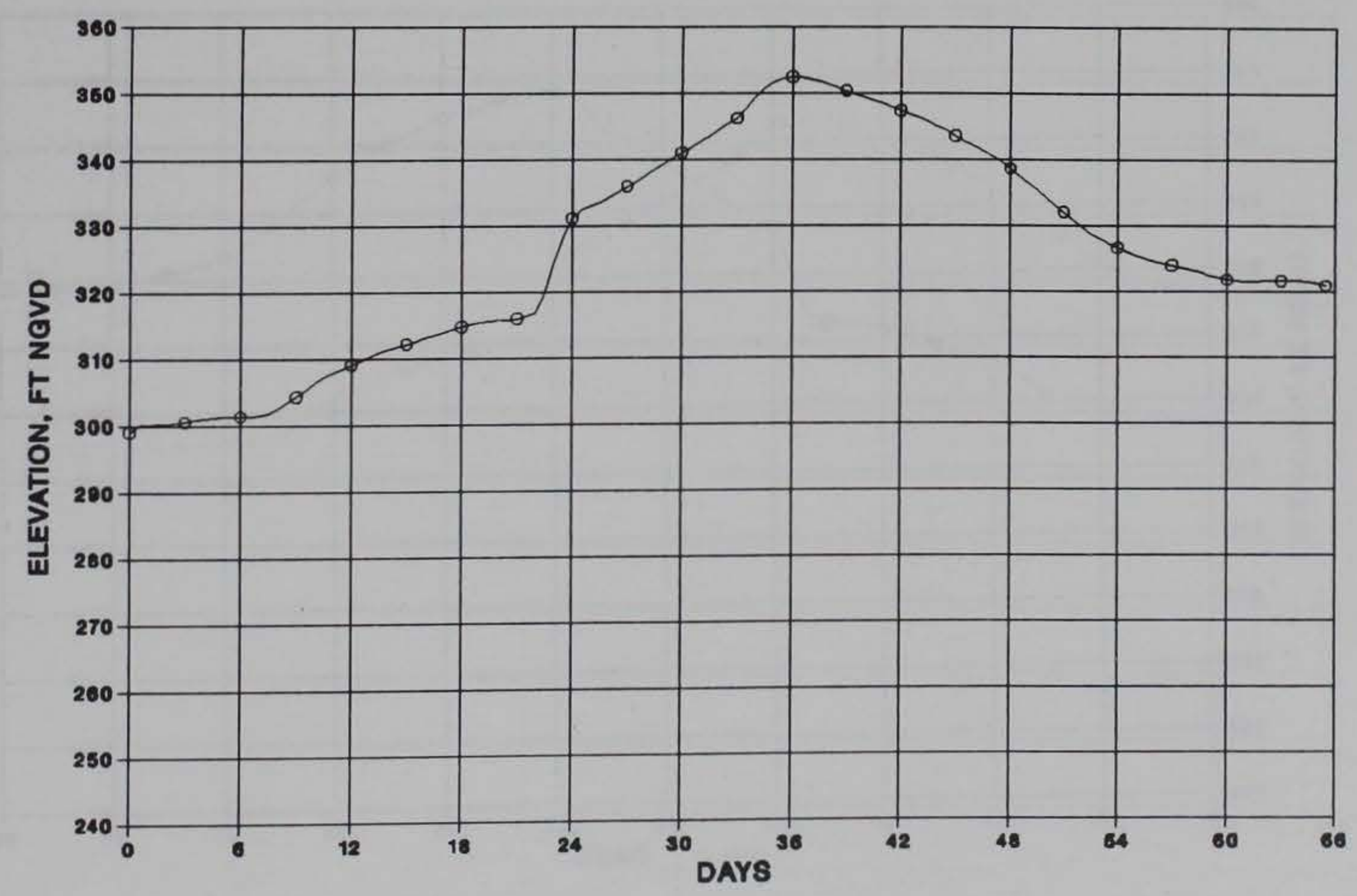

b. Elevation

Figure 87. Results at Paducah, KY, for Wheeler PMF dam break 


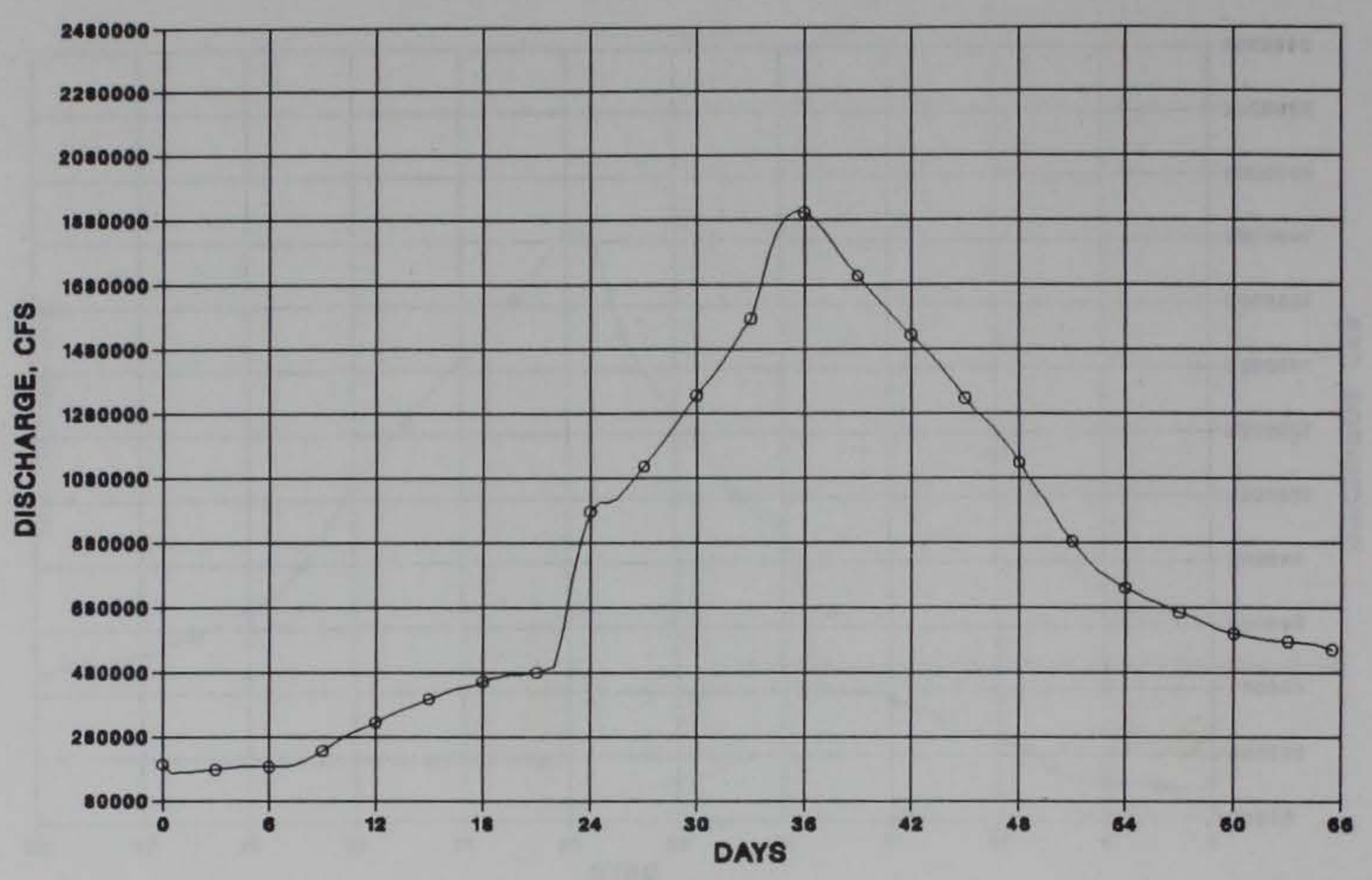

a. Discharge

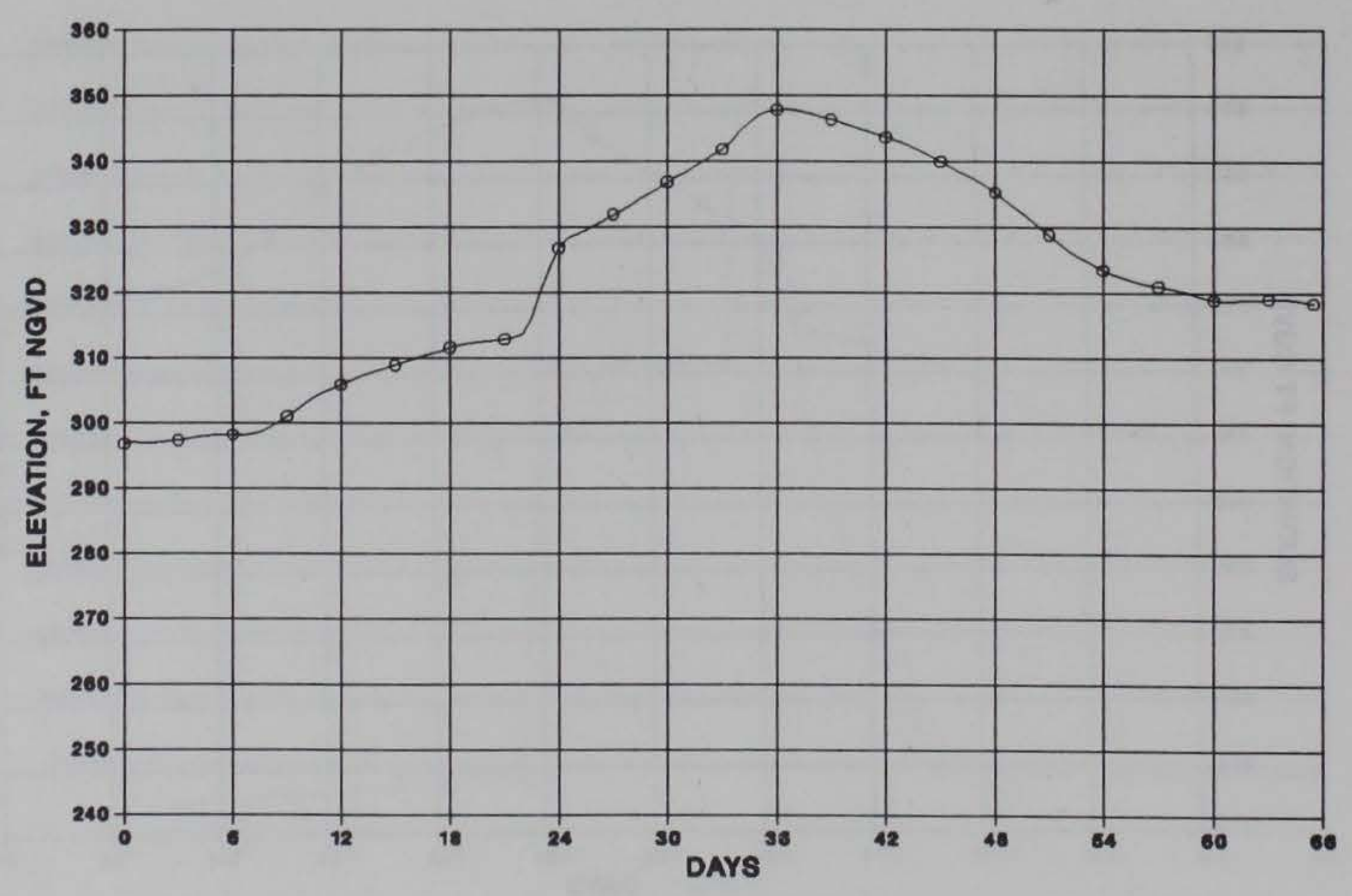

b. Elevation

Figure 88. Results at Metropolis, IL, for Wheeler PMF dam break 


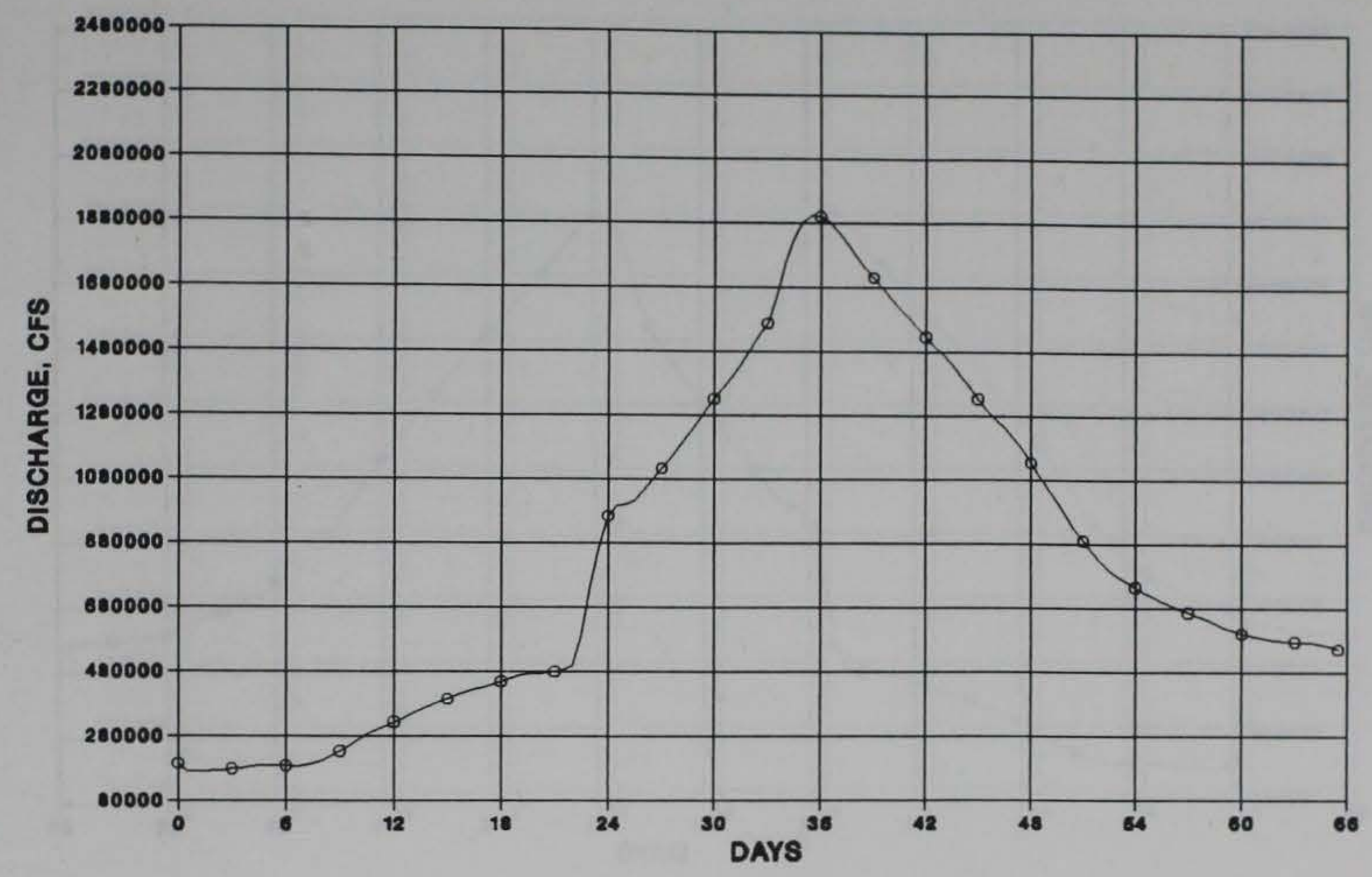

a. Discharge

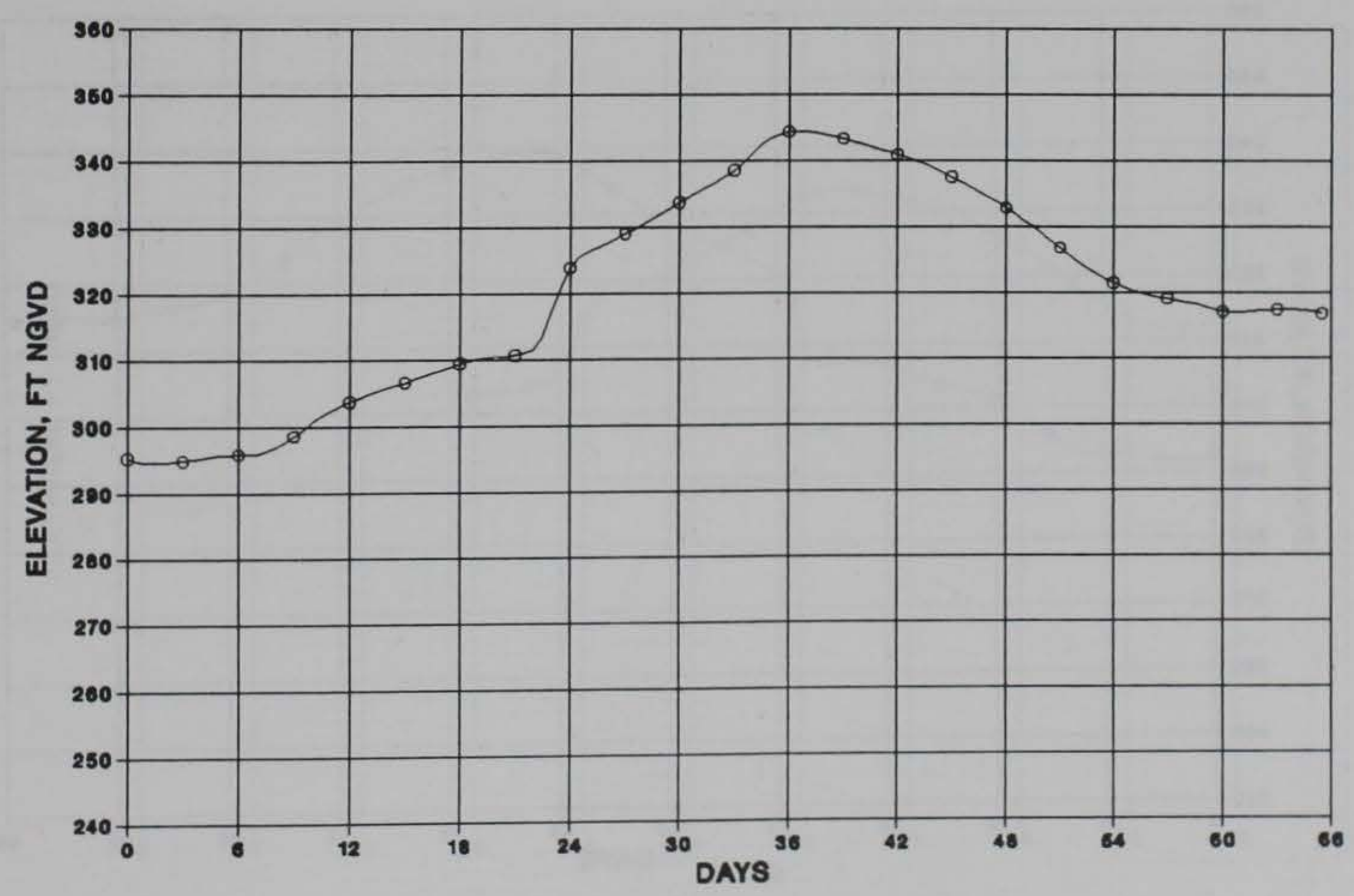

b. Elevation

Figure 89. Results at Joppa, IL, for Wheeler PMF dam break 


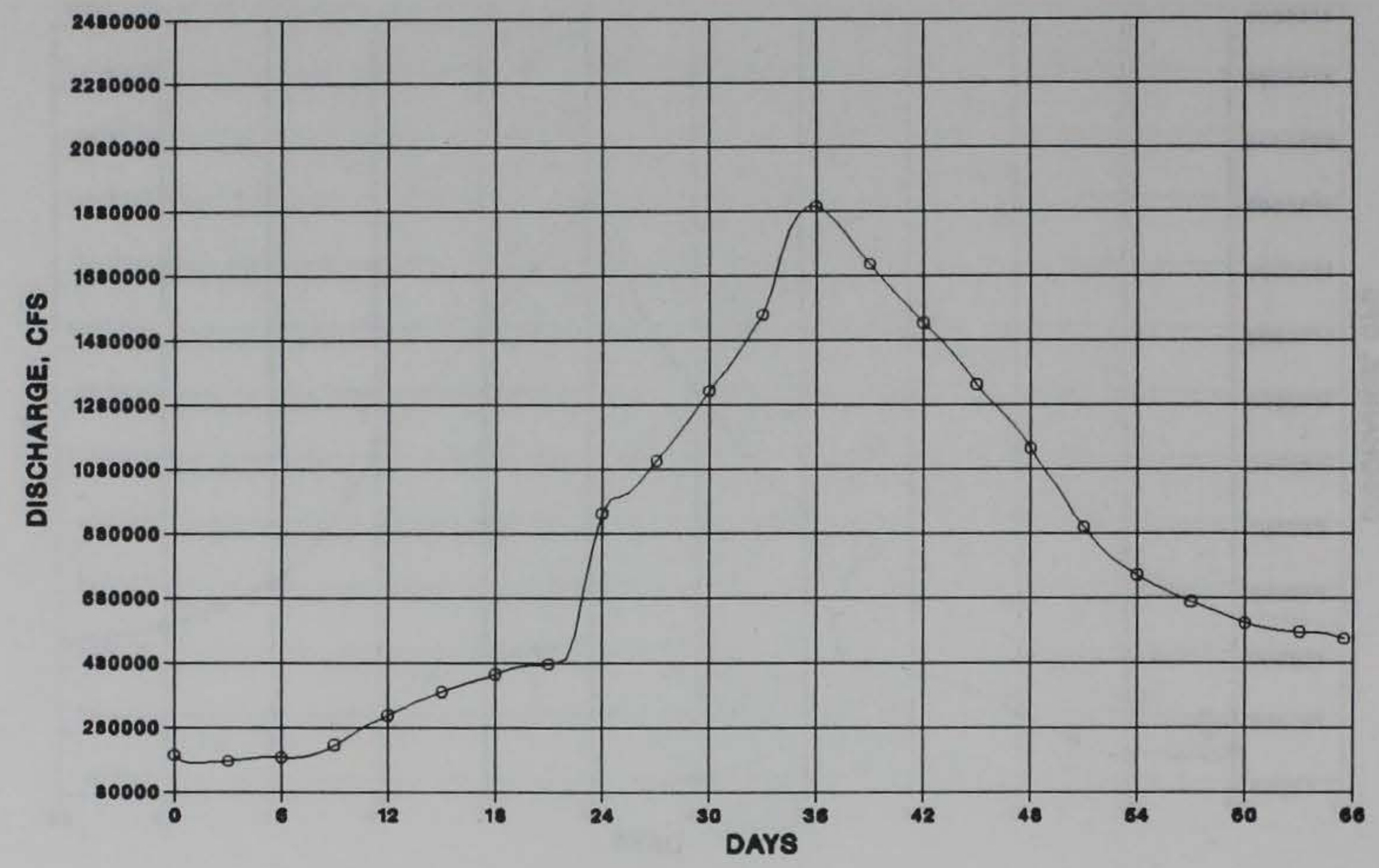

a. Discharge

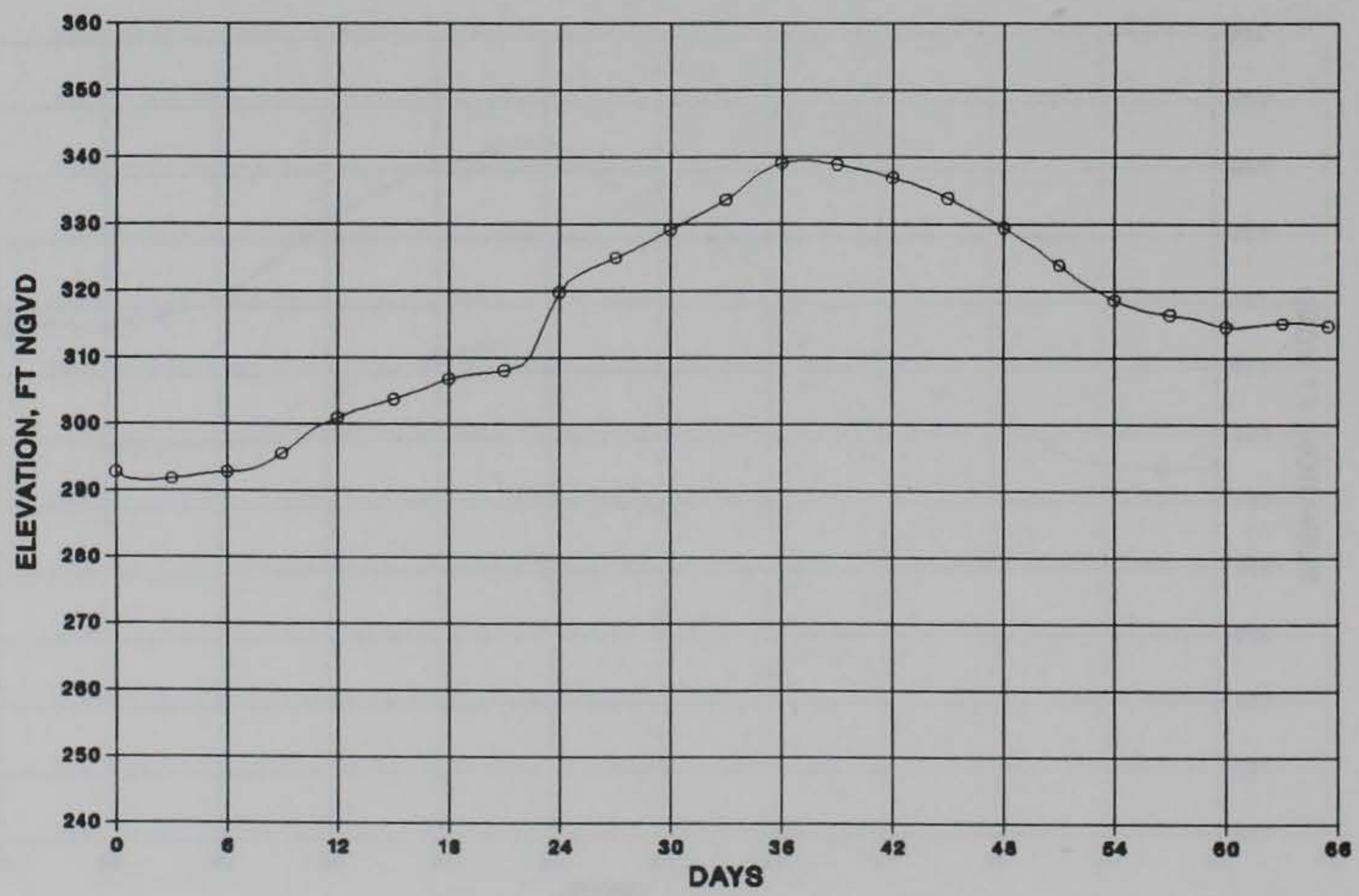

b. Elevation

Figure 90. Results at Olmstead, IL, for Wheeler PMF dam break 


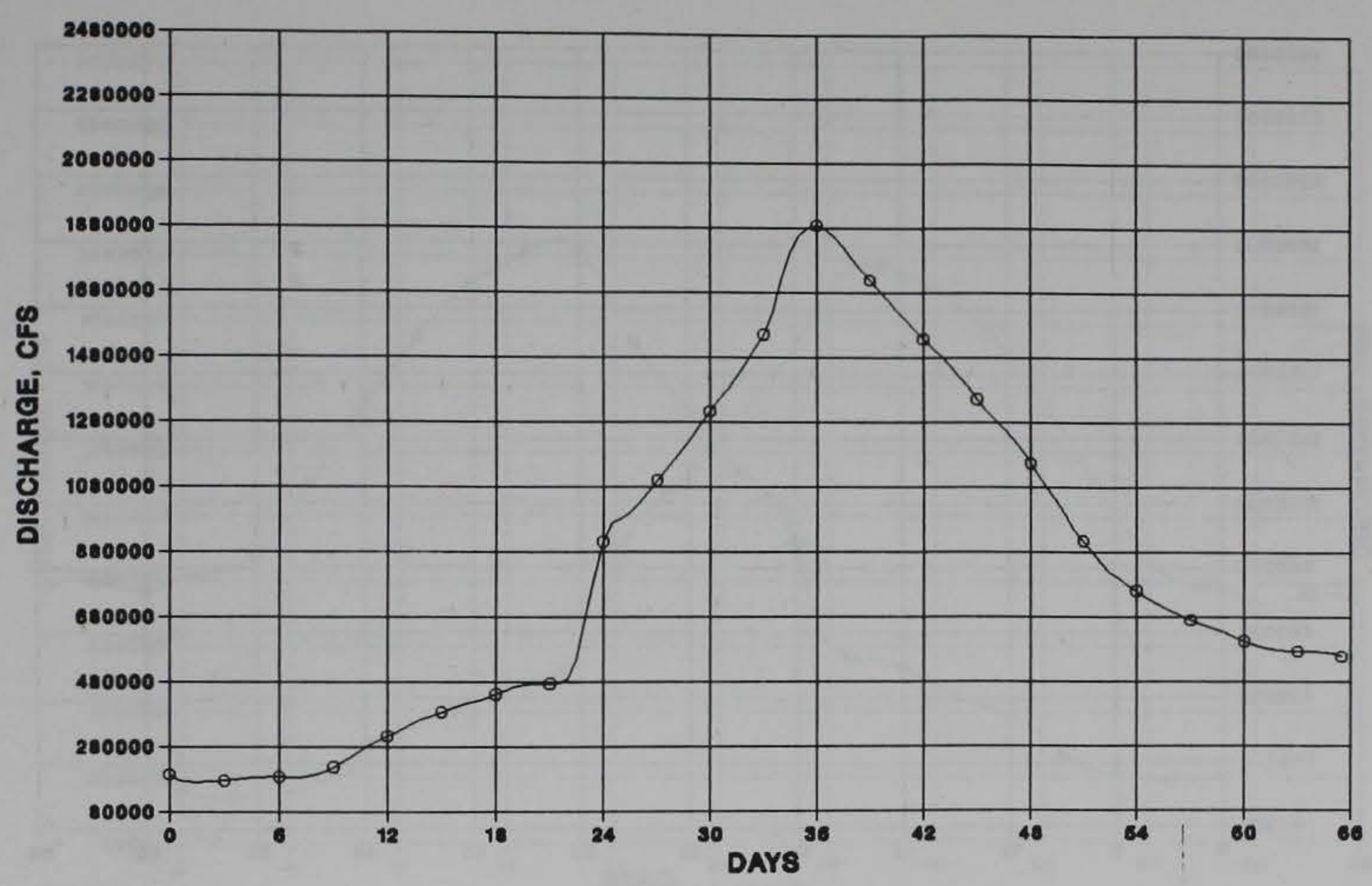

a. Discharge

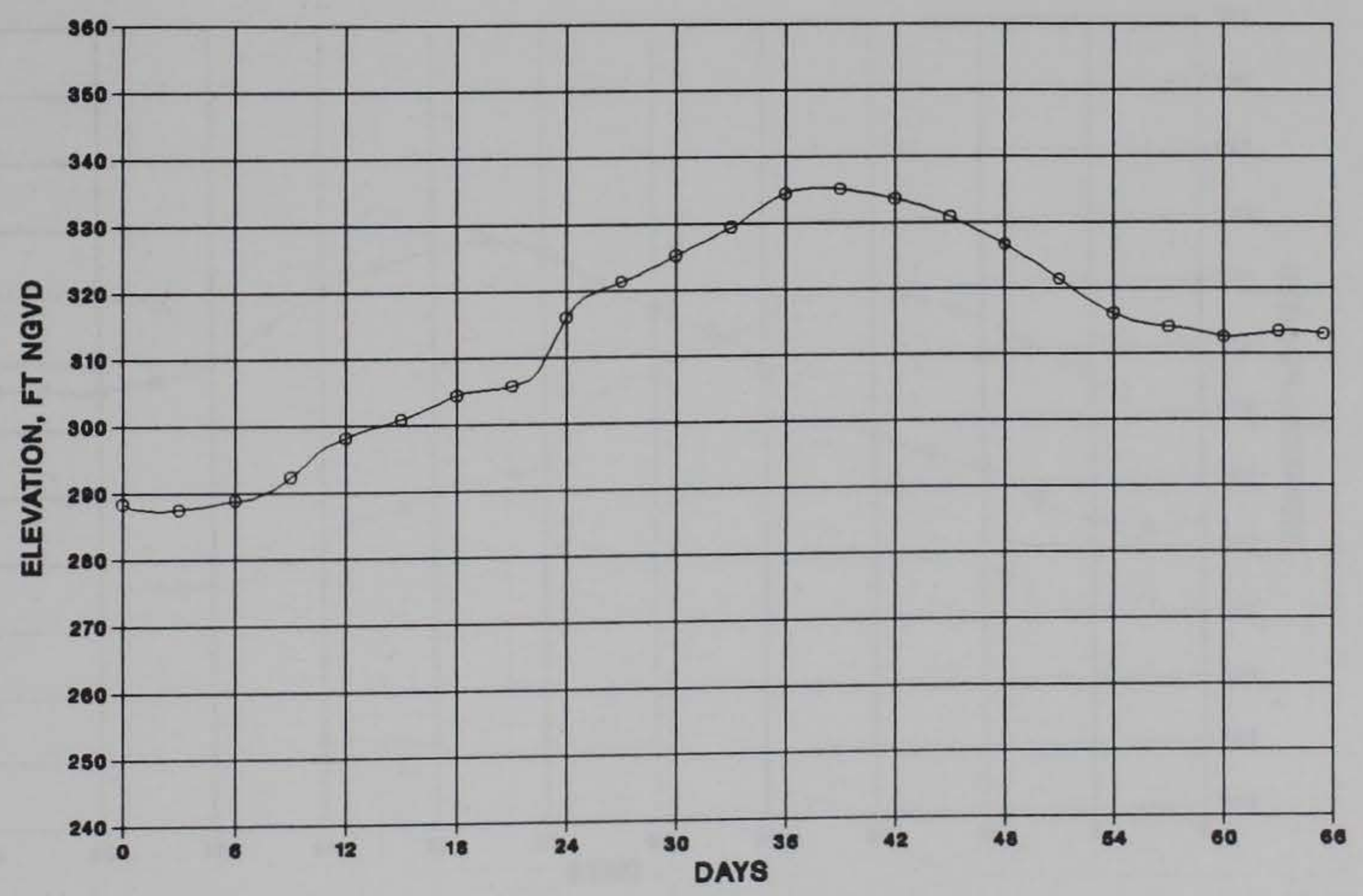

b. Elevation

Figure 91. Results at Cairo, IL, for Wheeler PMF dam break 


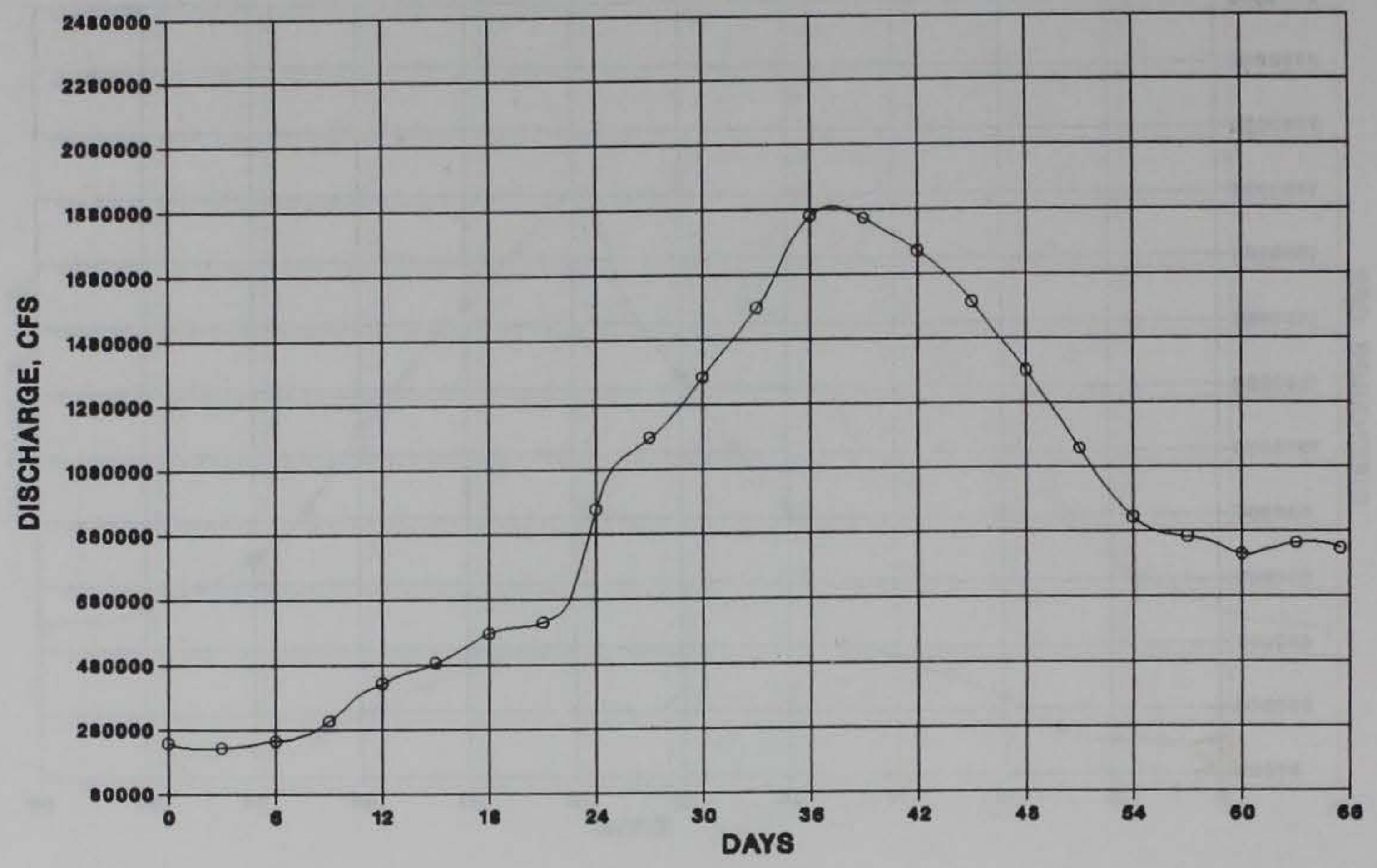

a. Discharge

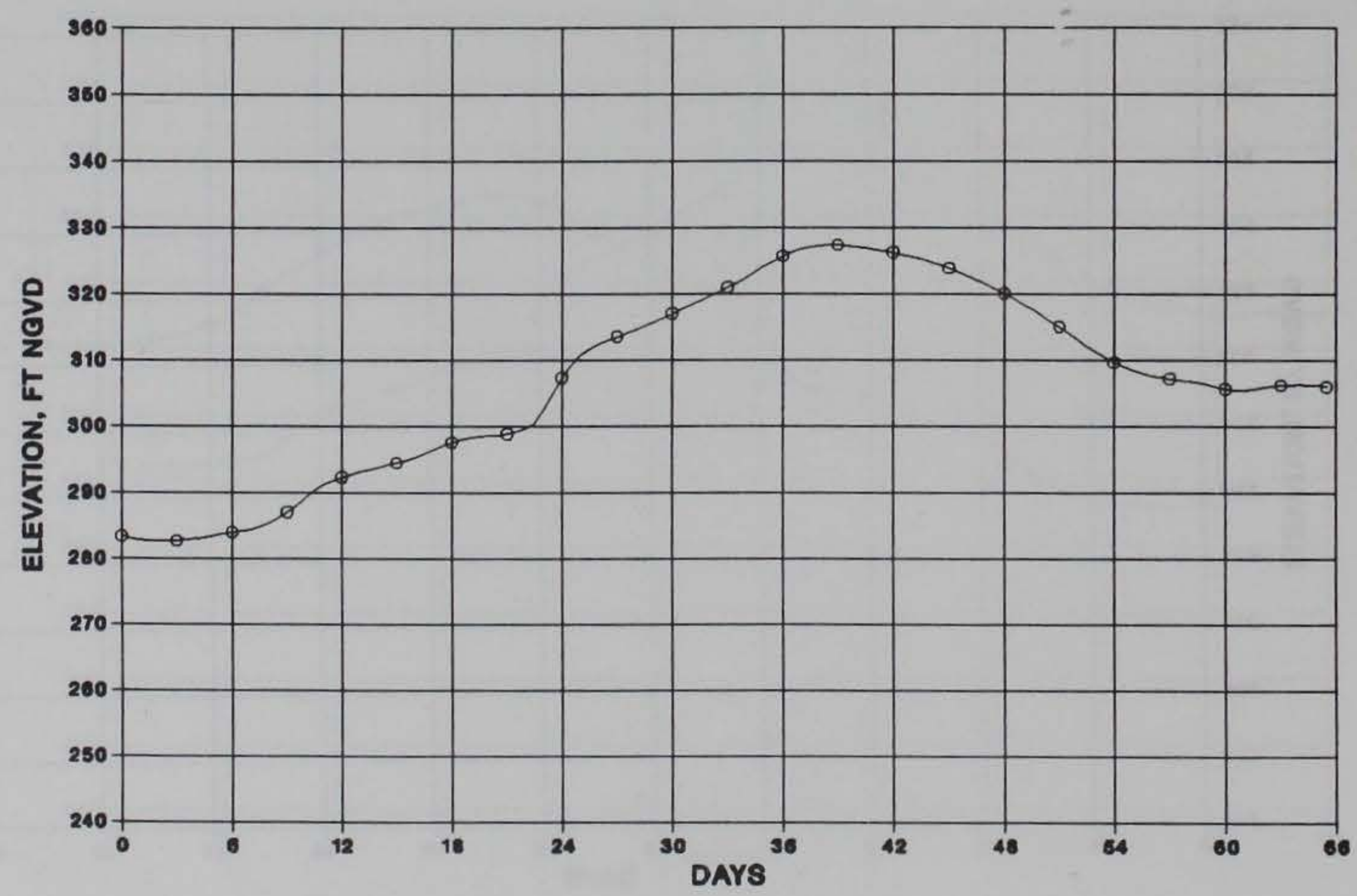

b. Elevation

Figure 92. Results at Columbus, KY, for Wheeler PMF dam break 


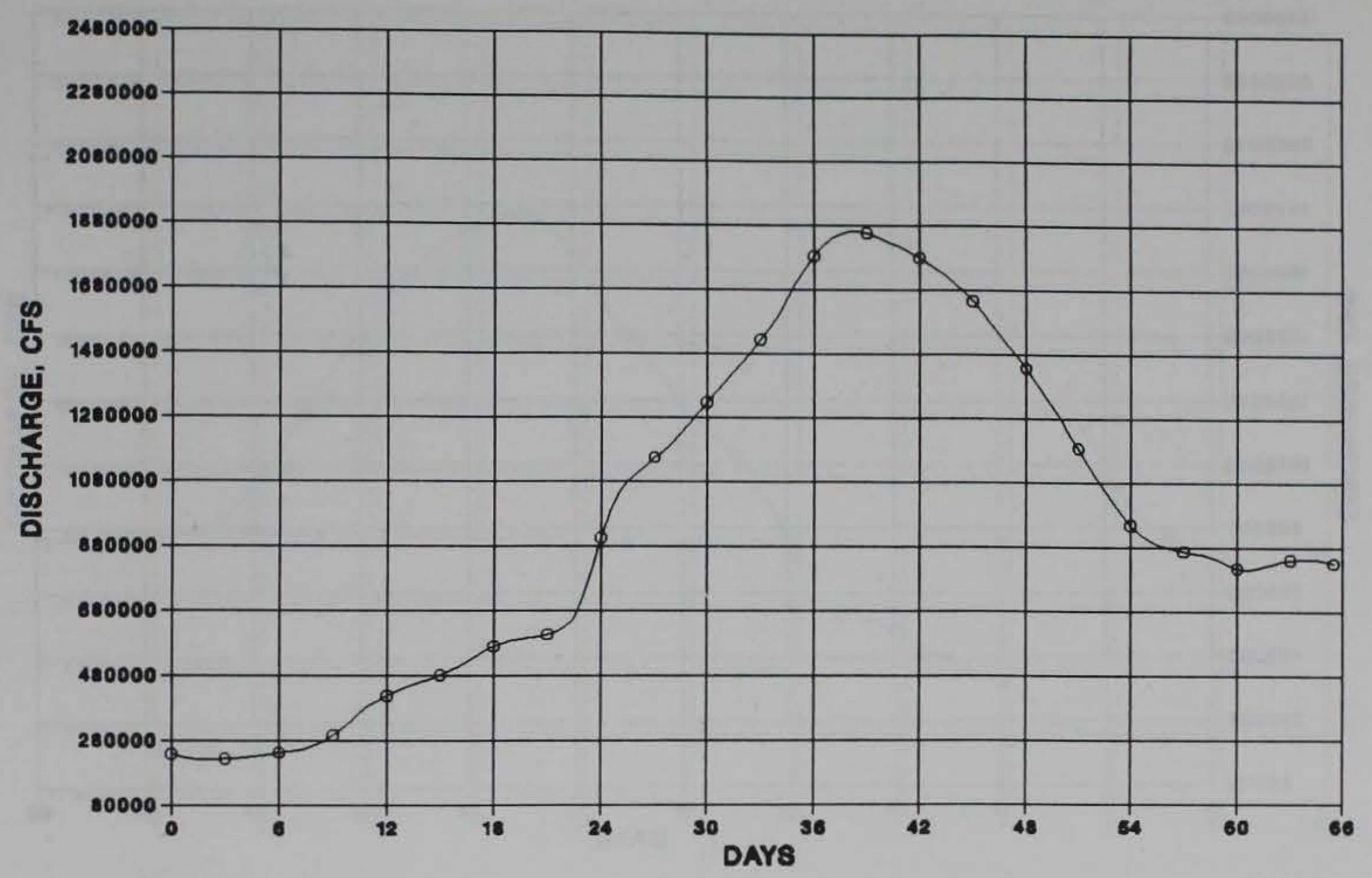

a. Discharge

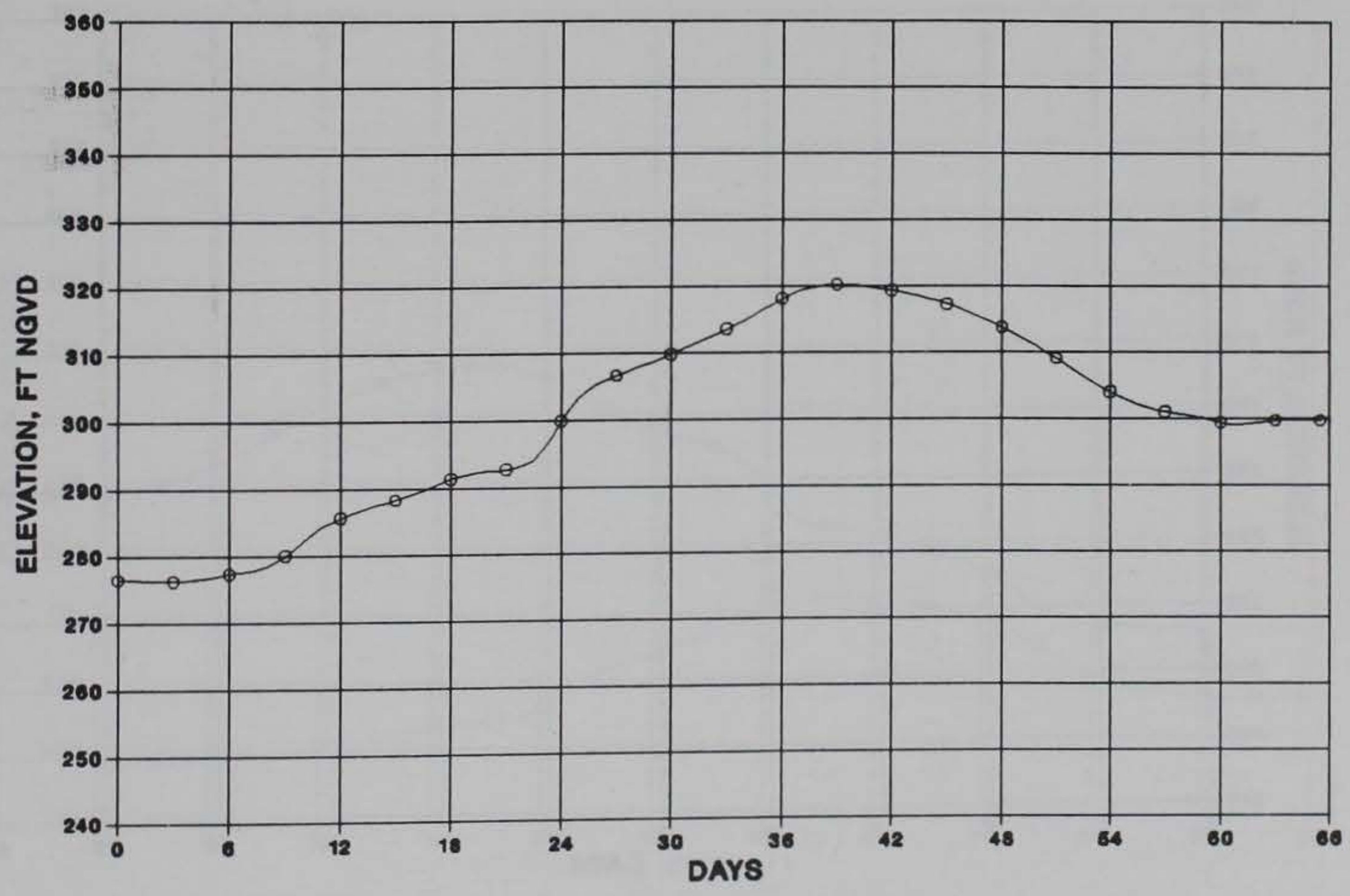

b. Elevation

Figure 93. Results at Hickman, KY, for Wheeler PMF dam break 


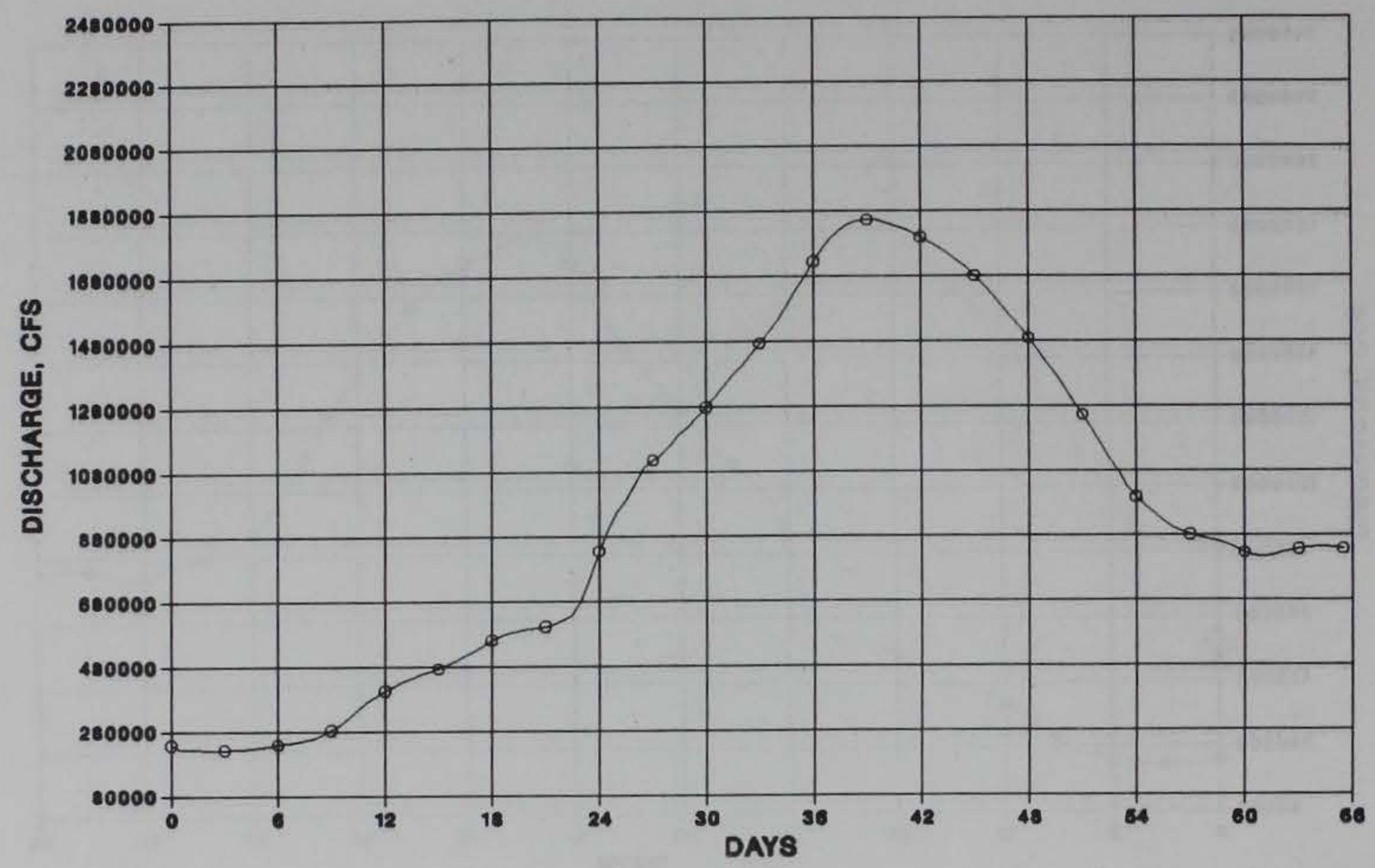

a. Discharge

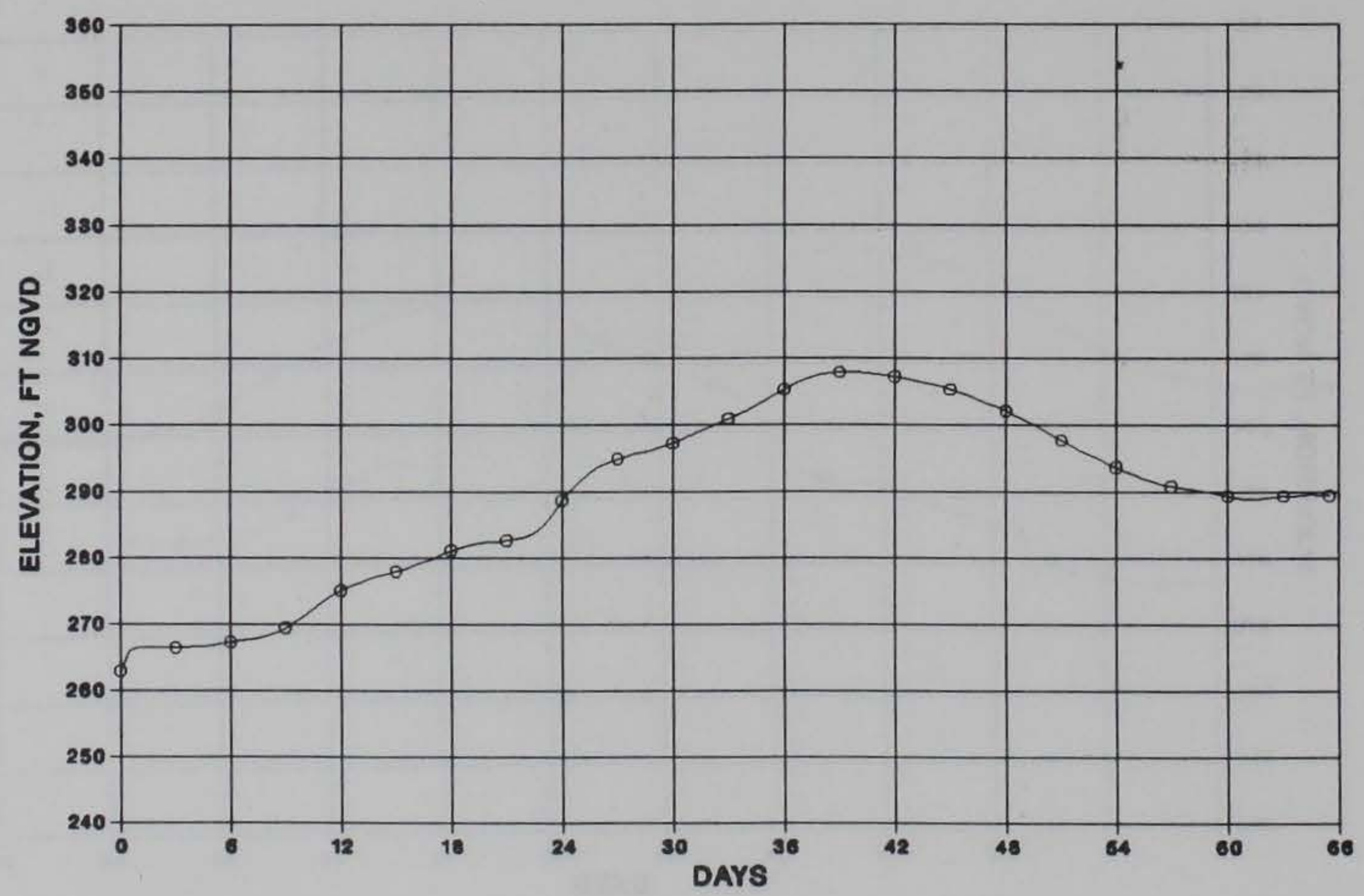

b. Elevation

Figure 94. Results at New Madrid, MO, for Wheeler PMF dam break 


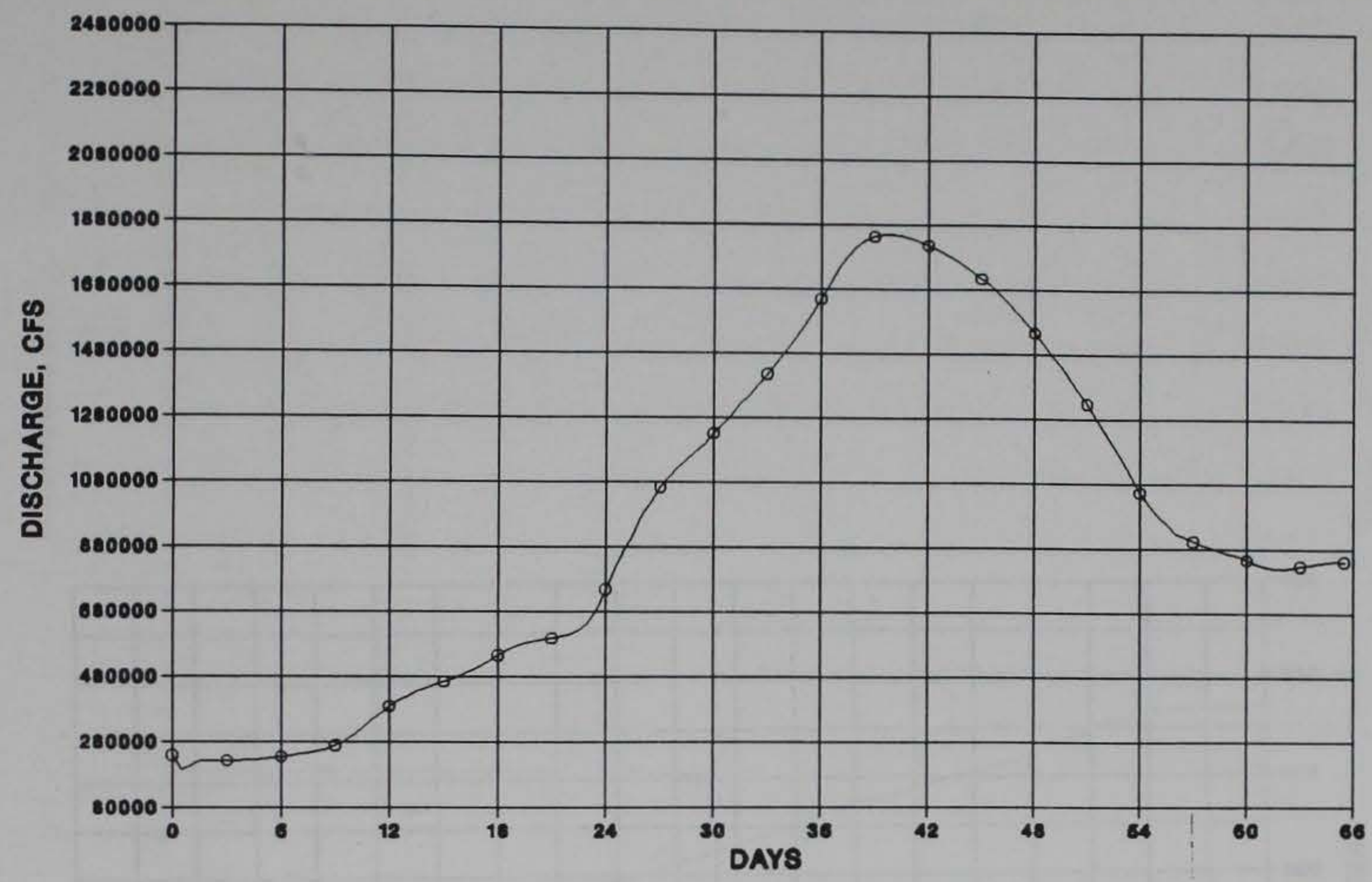

a. Discharge

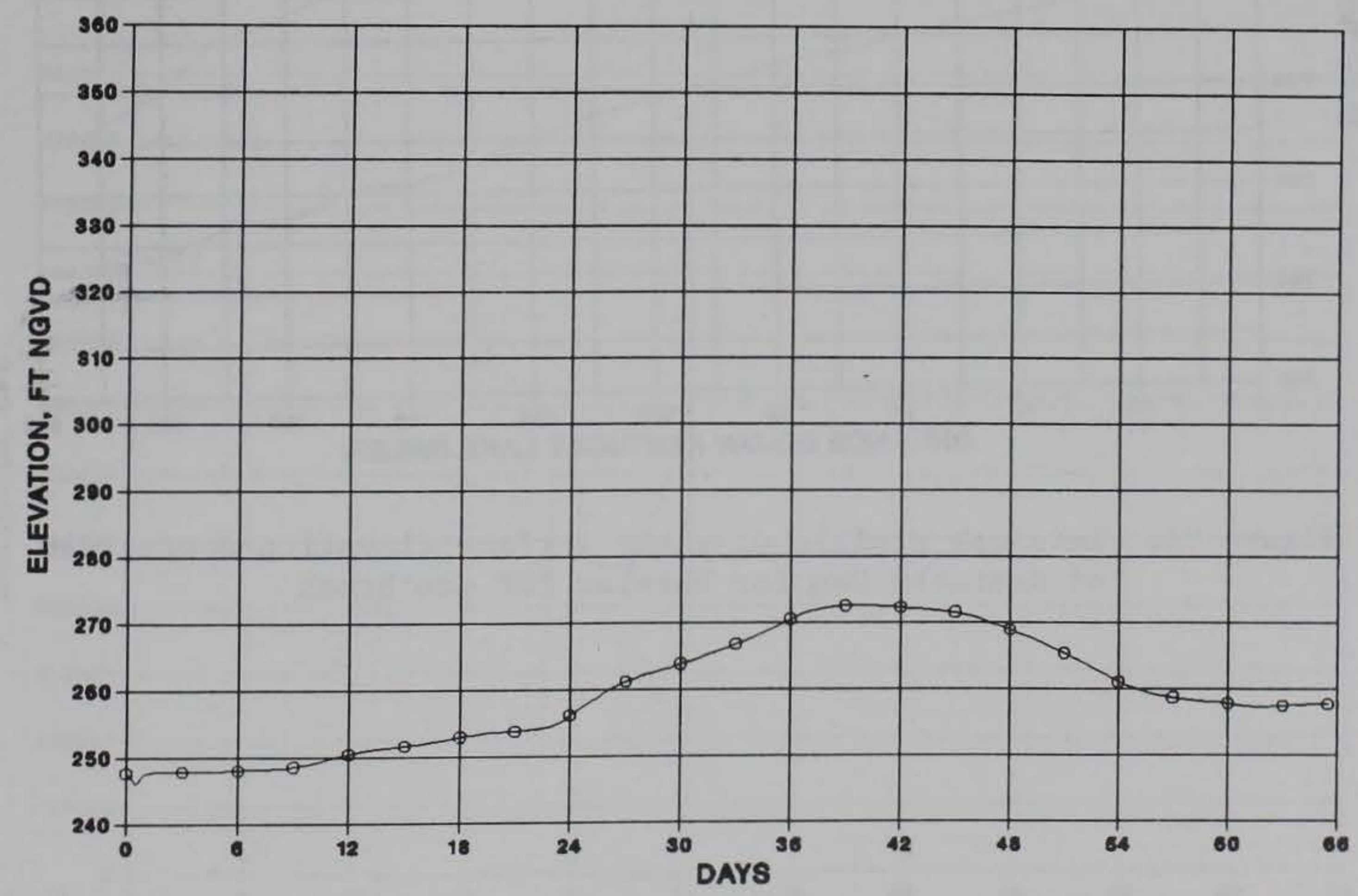

b. Elevation

Figure 95. Results at Caruthersville, MO, for Wheeler PMF dam break 


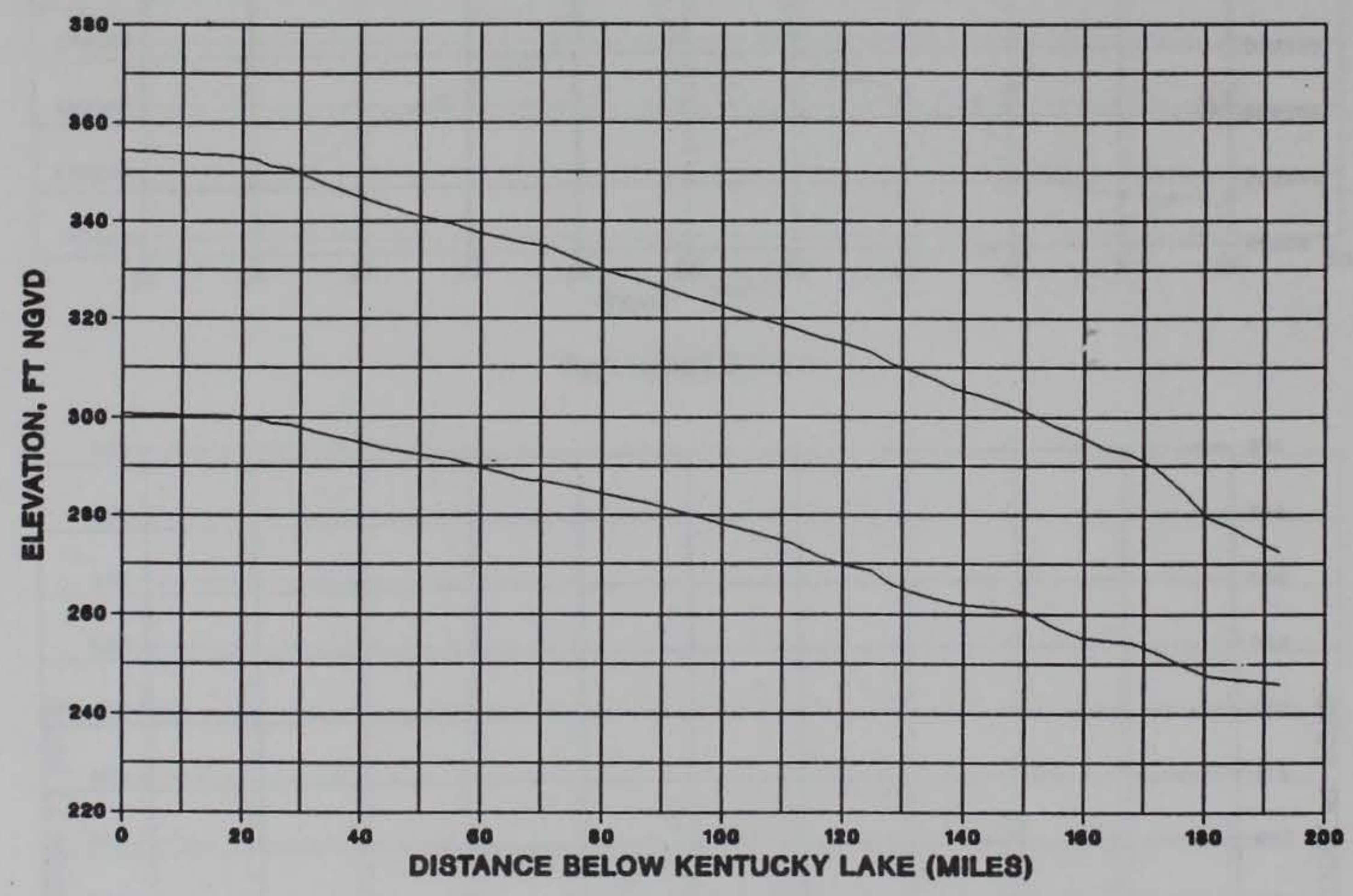

Figure 96. Envelope profile of water surface elevation downstream of Kentucky Dam for Wheeler PMF dam break 


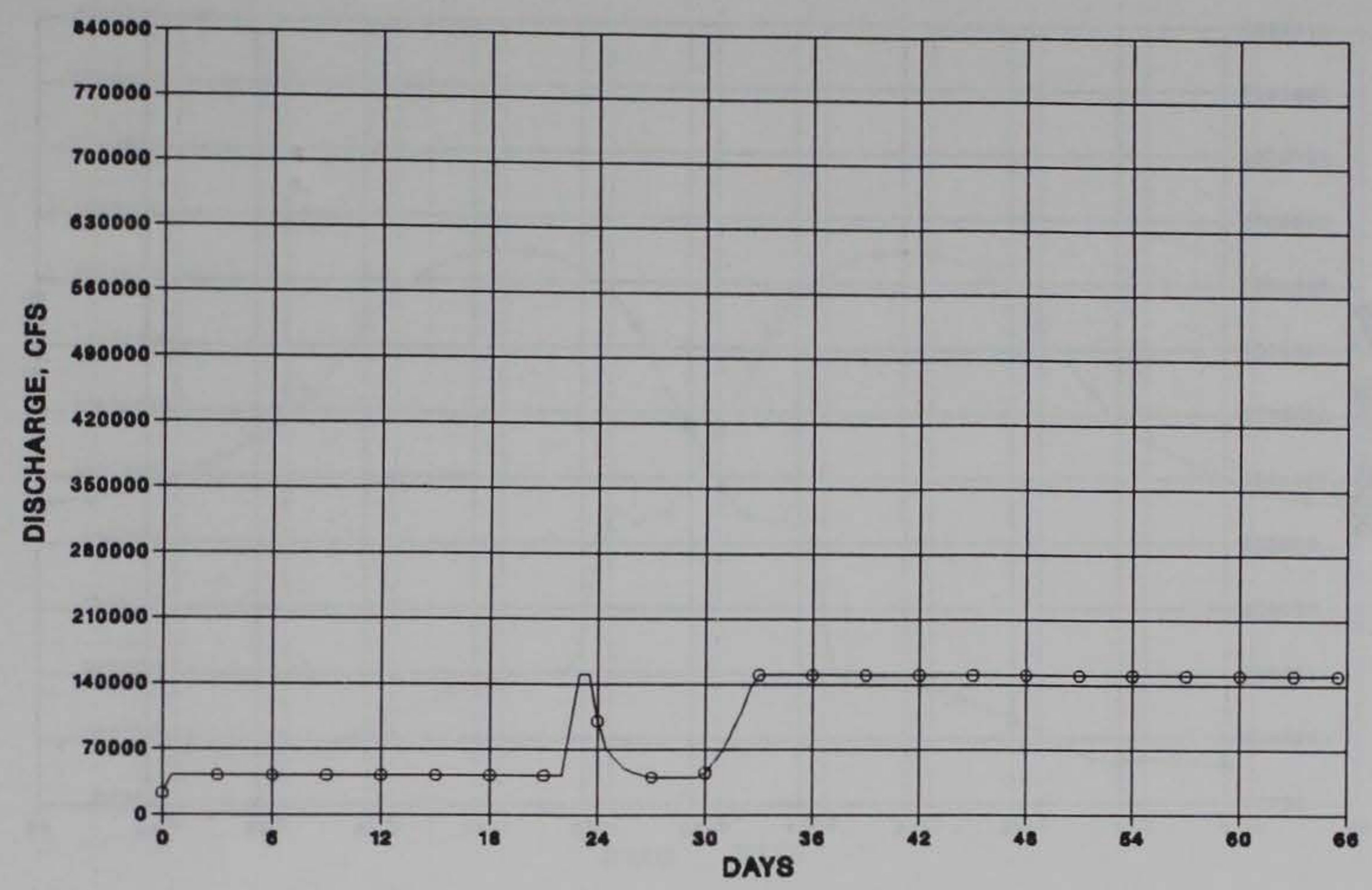

Figure 97. Discharge from Barkley Lake for Tellico PMF dam break

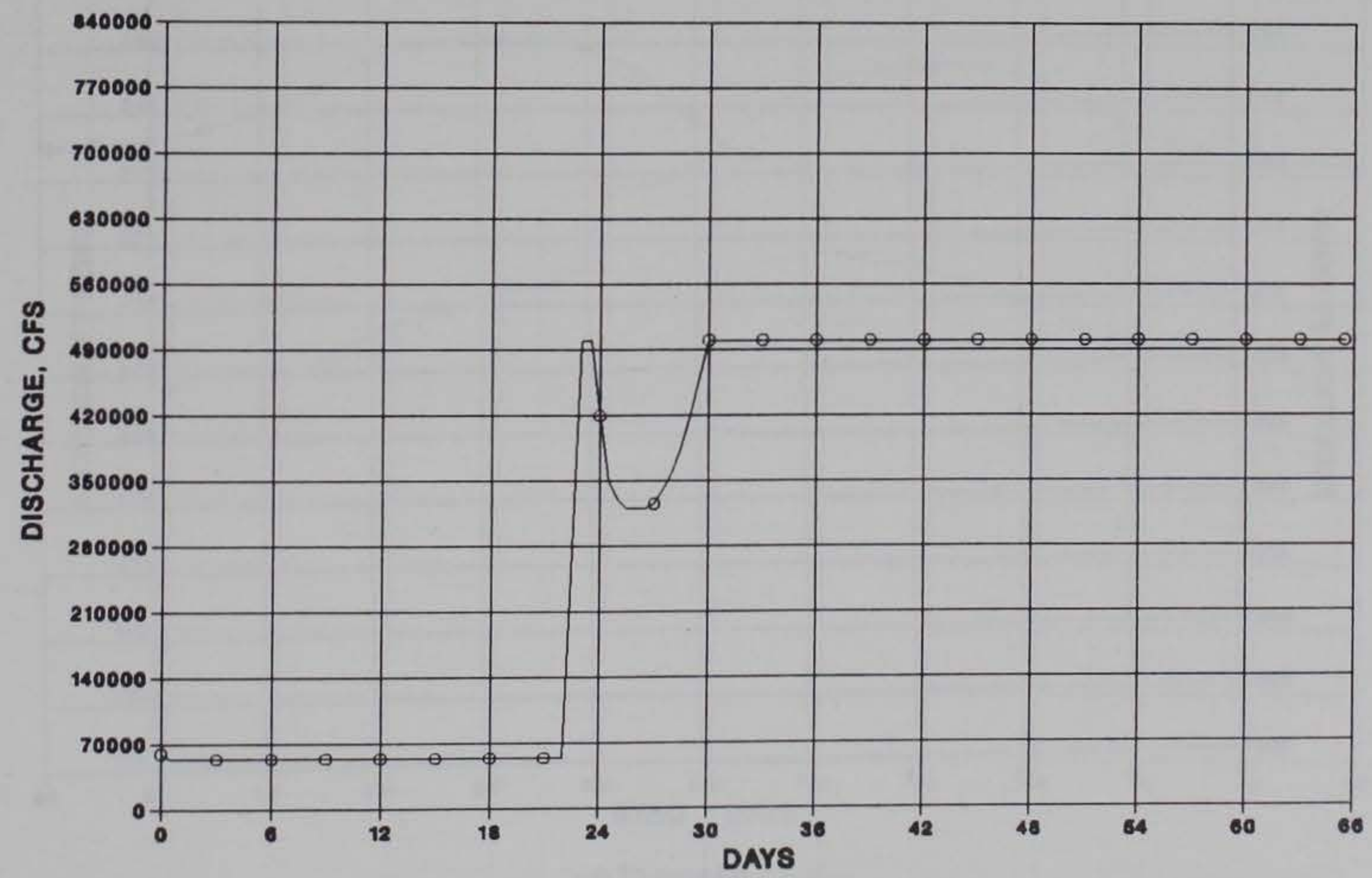

Figure 98. Discharge from Kentucky Lake for Tellico PMF dam break 


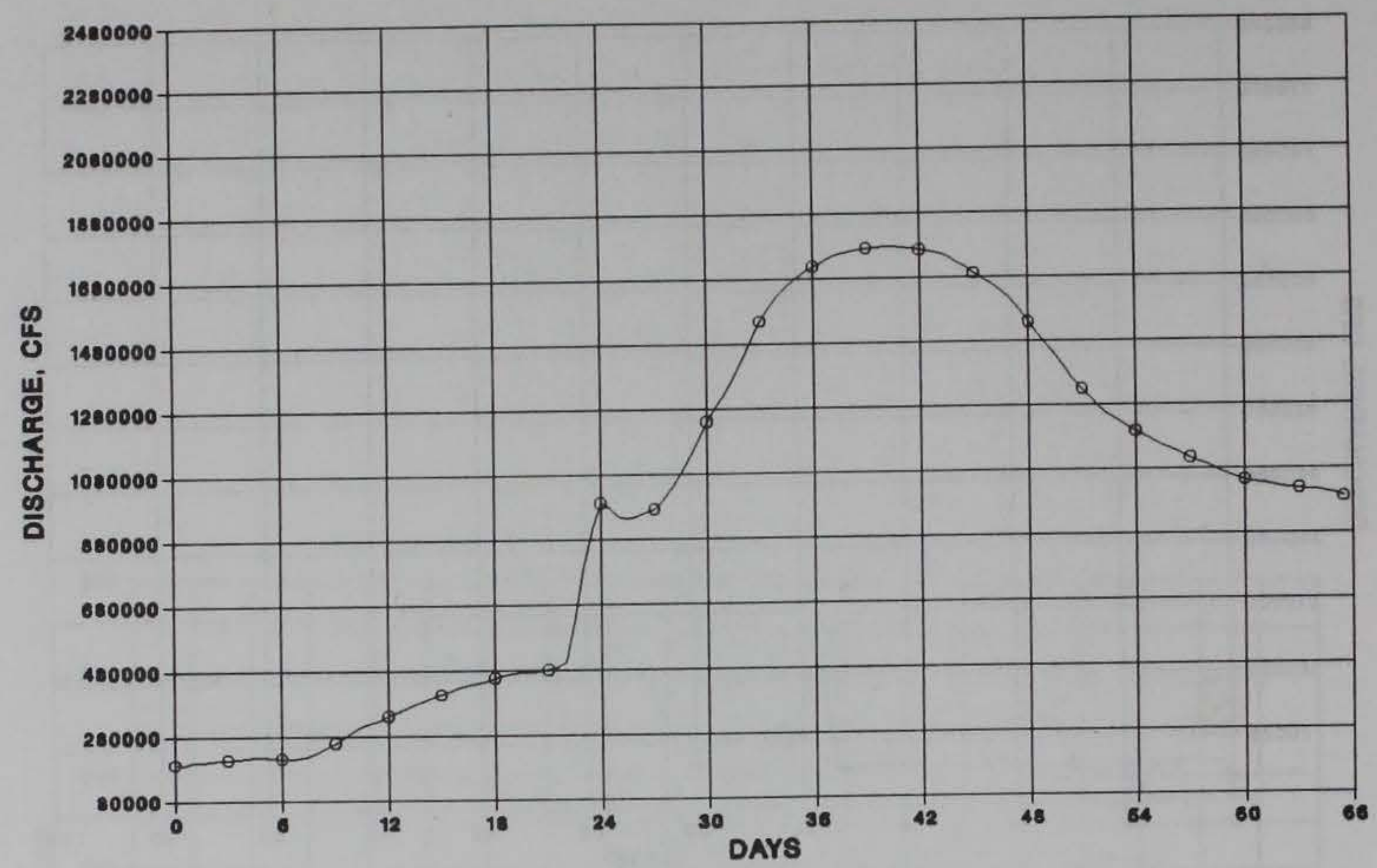

a. Discharge

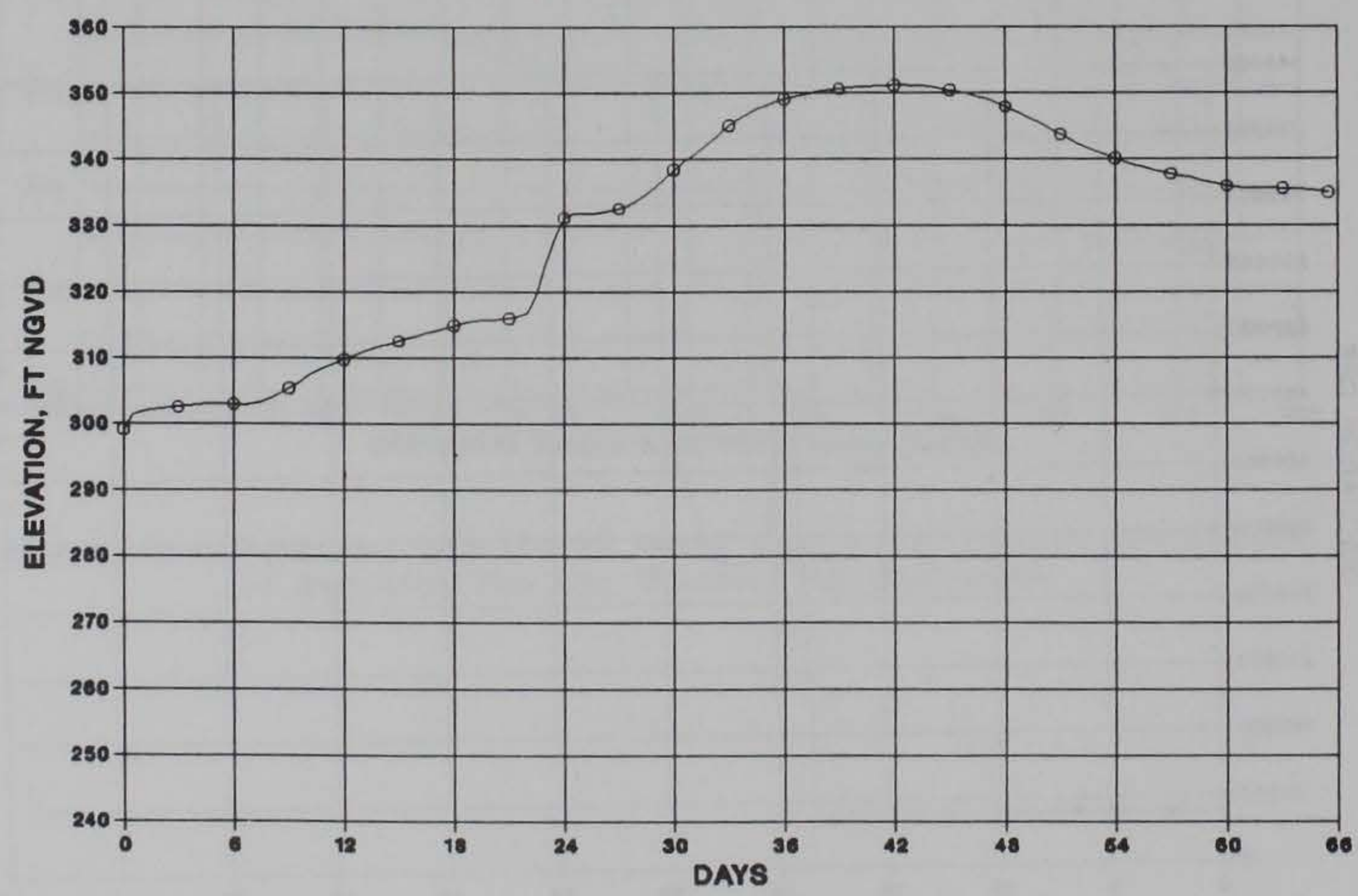

b. Elevation

Figure 99. Results at Paducah, KY, for Tellico PMF dam break 


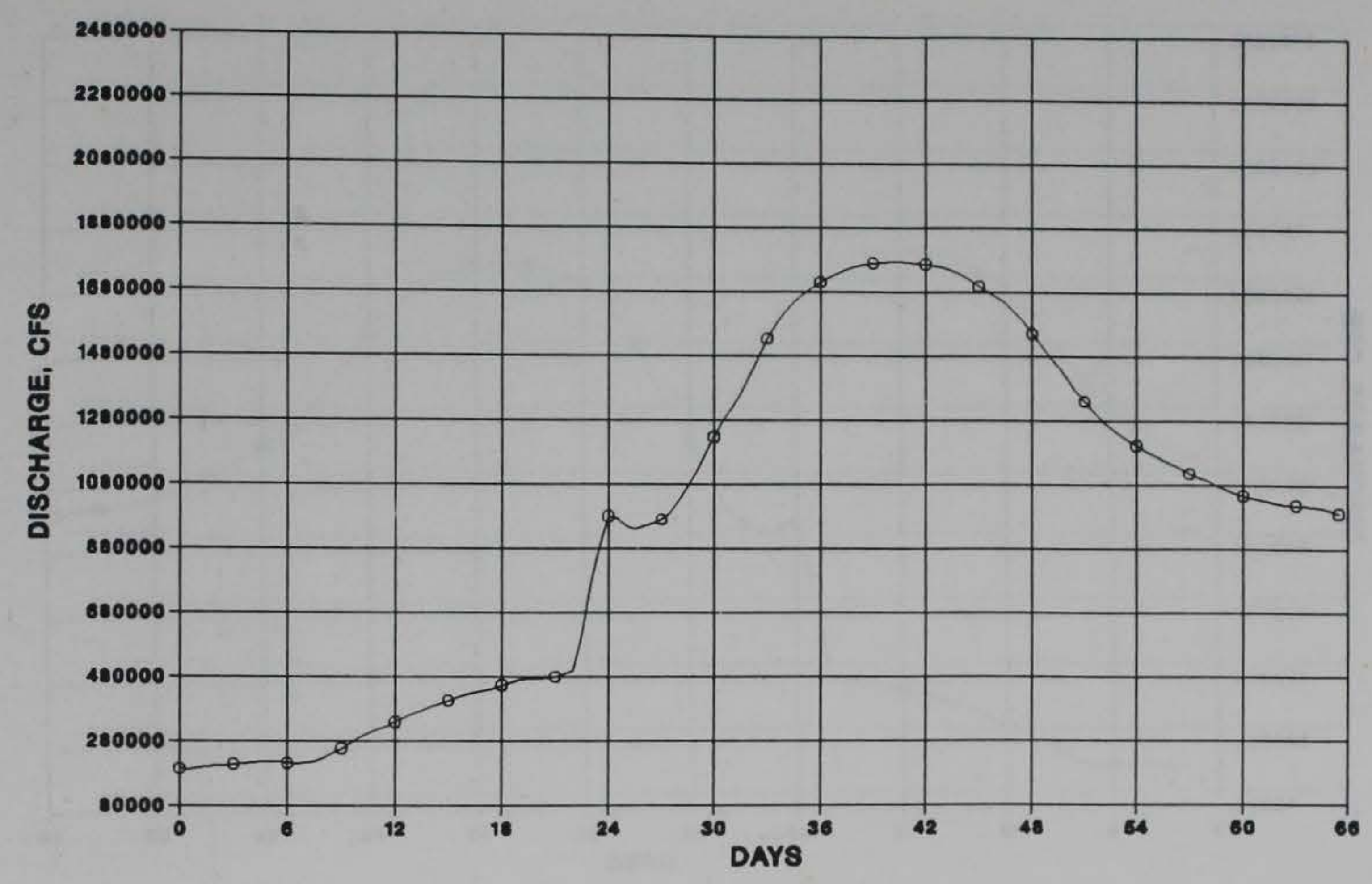

a. Discharge

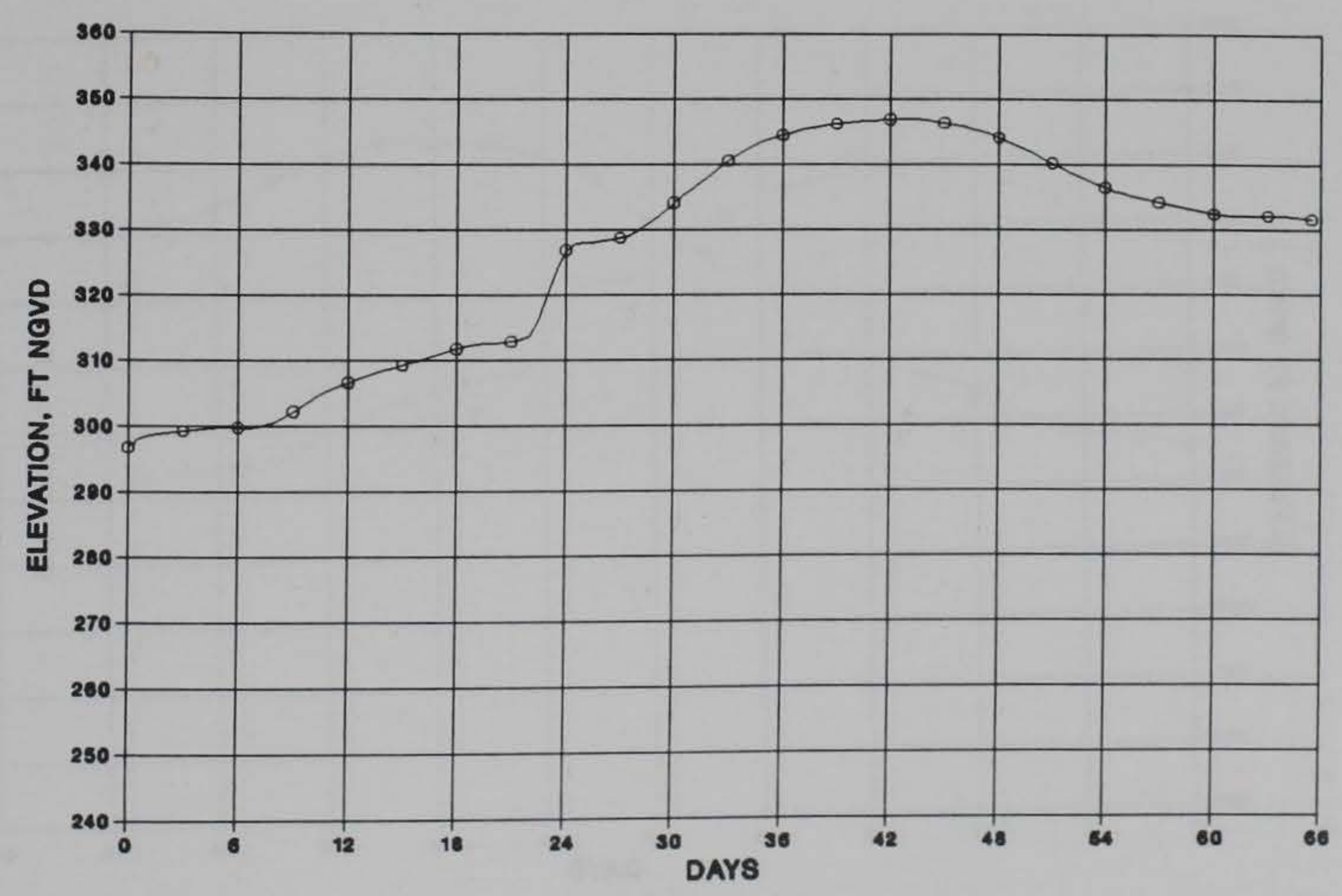

b. Elevation

Figure 100. Results at Metropolis, IL, for Tellico PMF dam break 


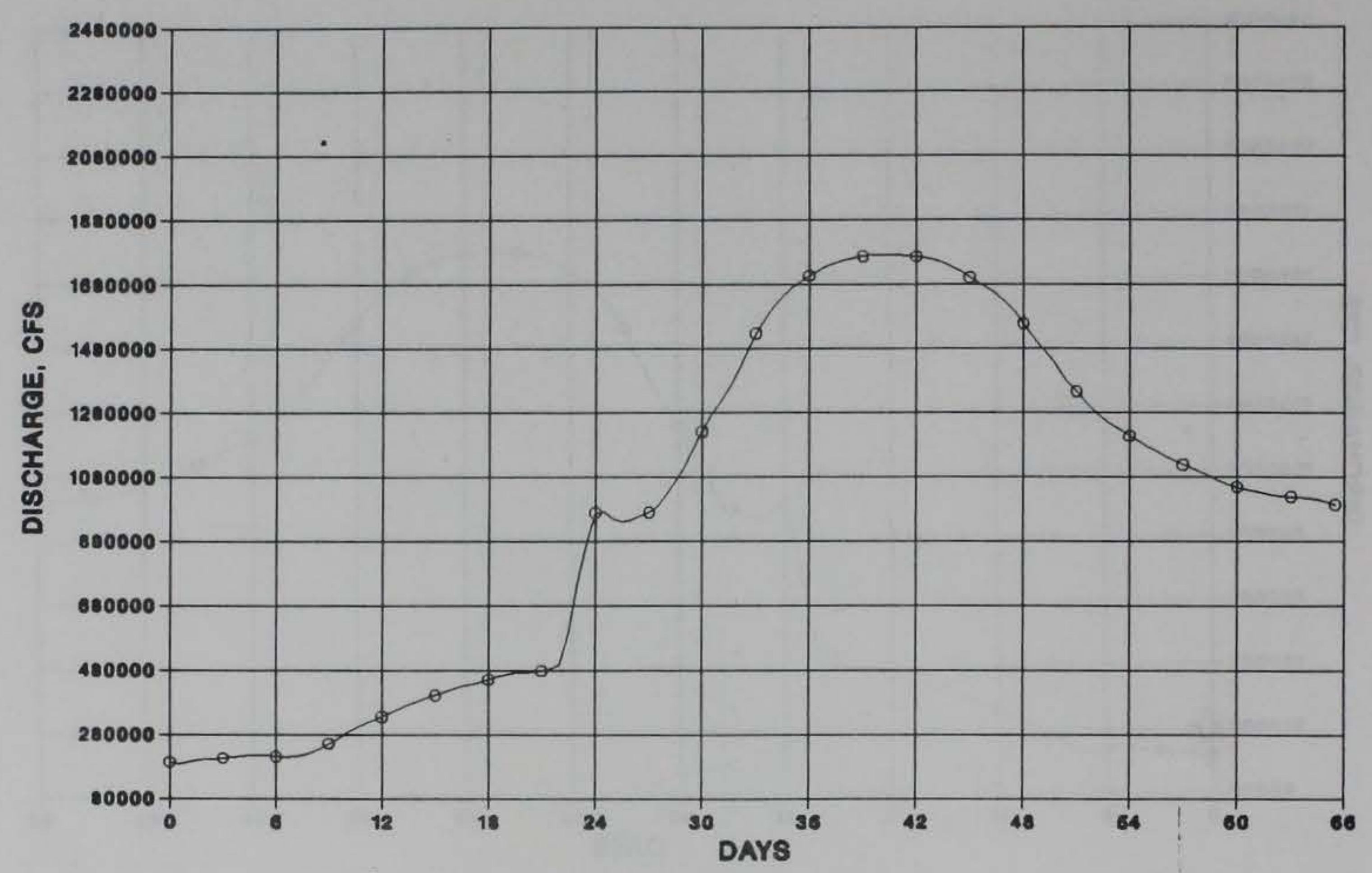

a. Discharge

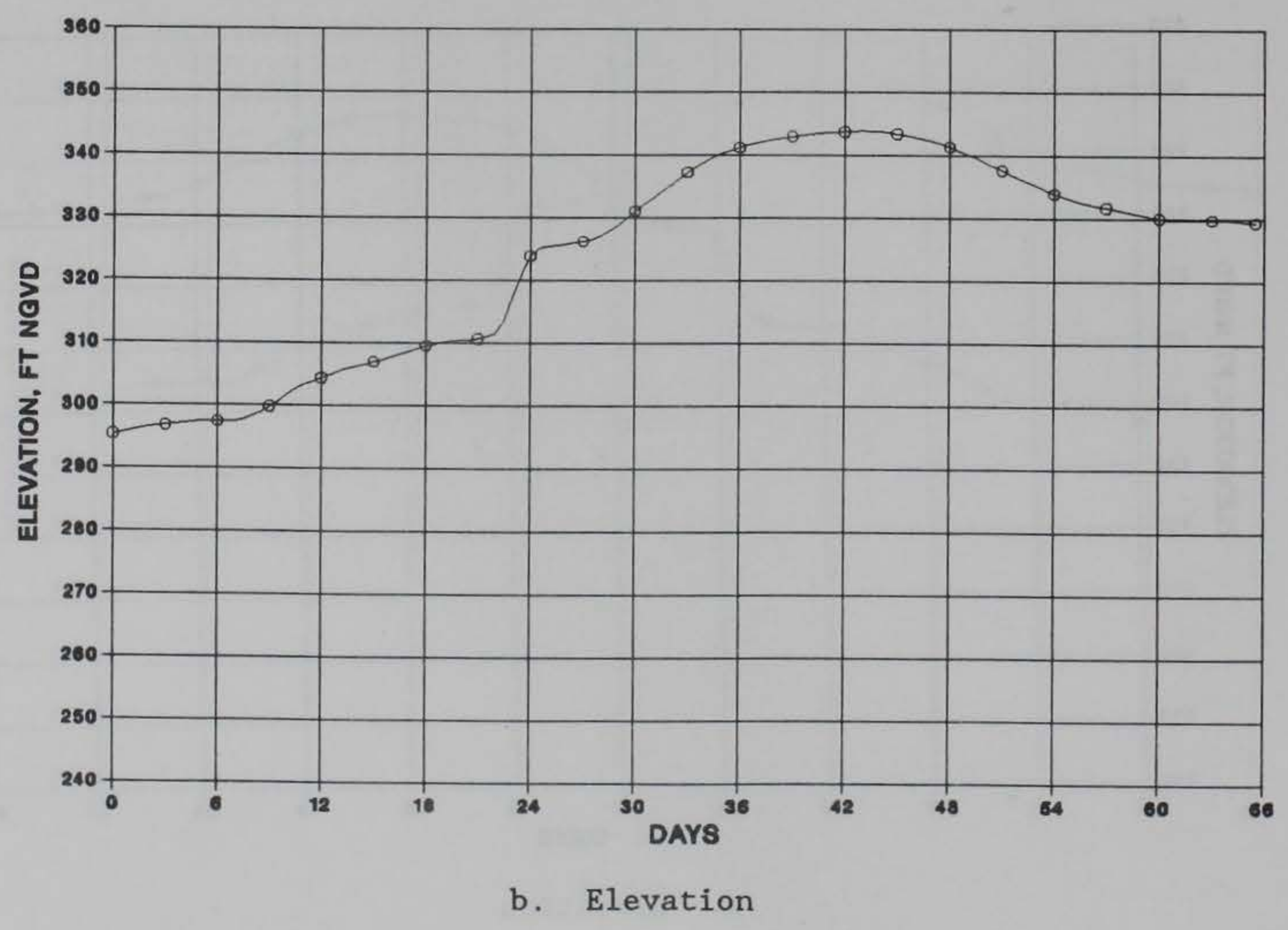

Figure 101. Results at Joppa, IL, for Tellico PMF dam break 


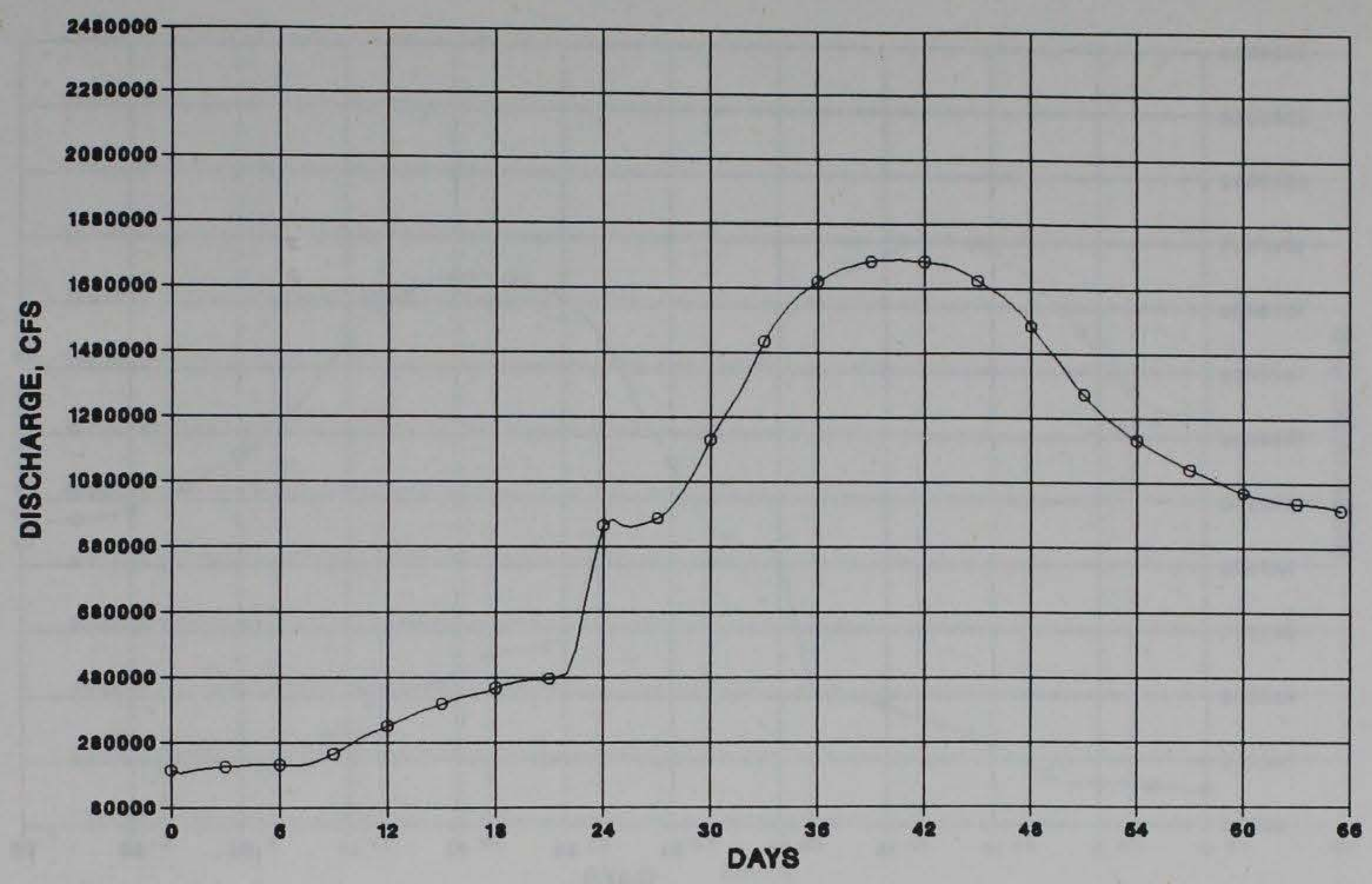

a. Discharge

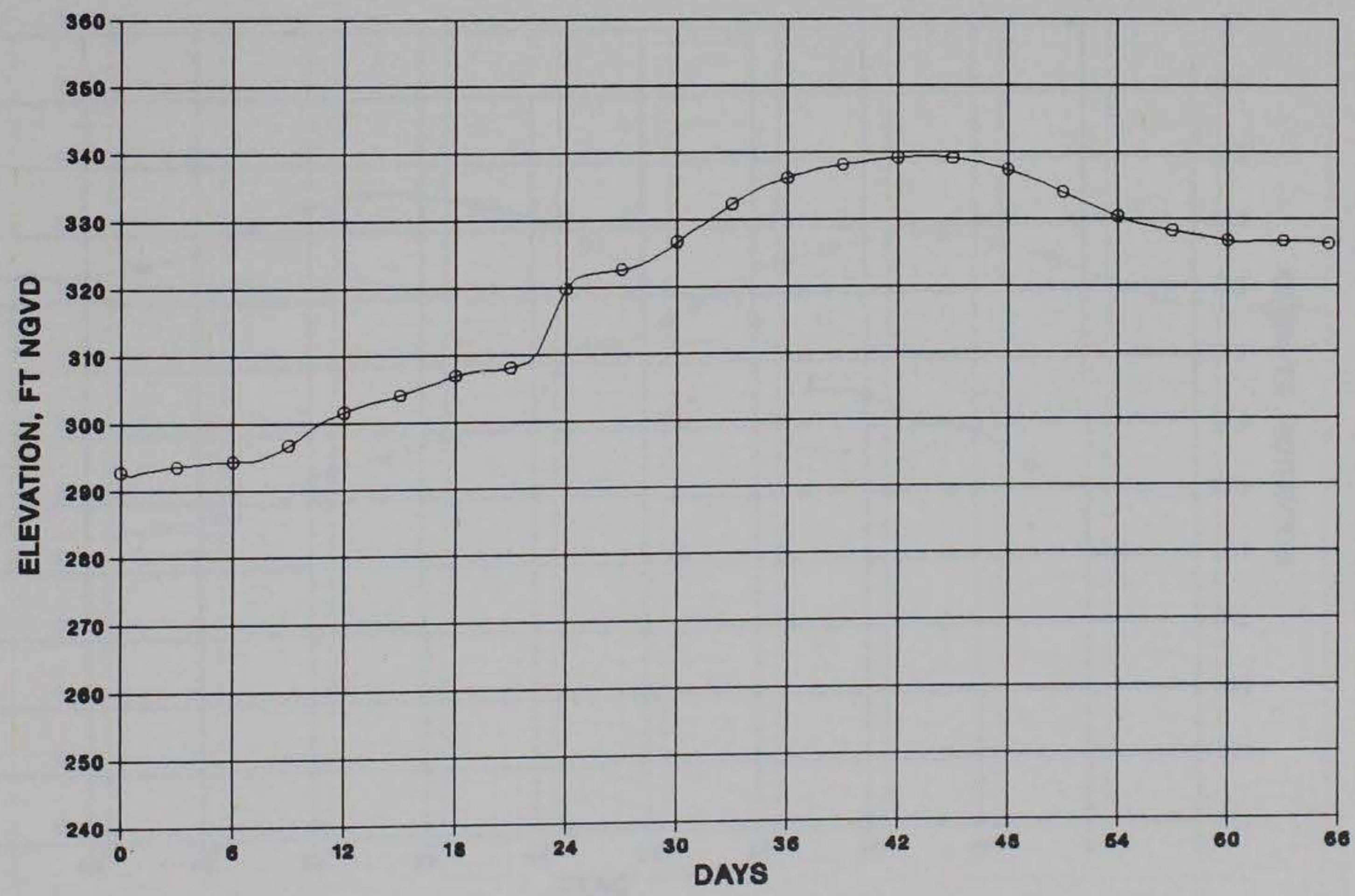

b. Elevation

Figure 102. Results at Olmstead, IL, for Tellico PMF dam break 


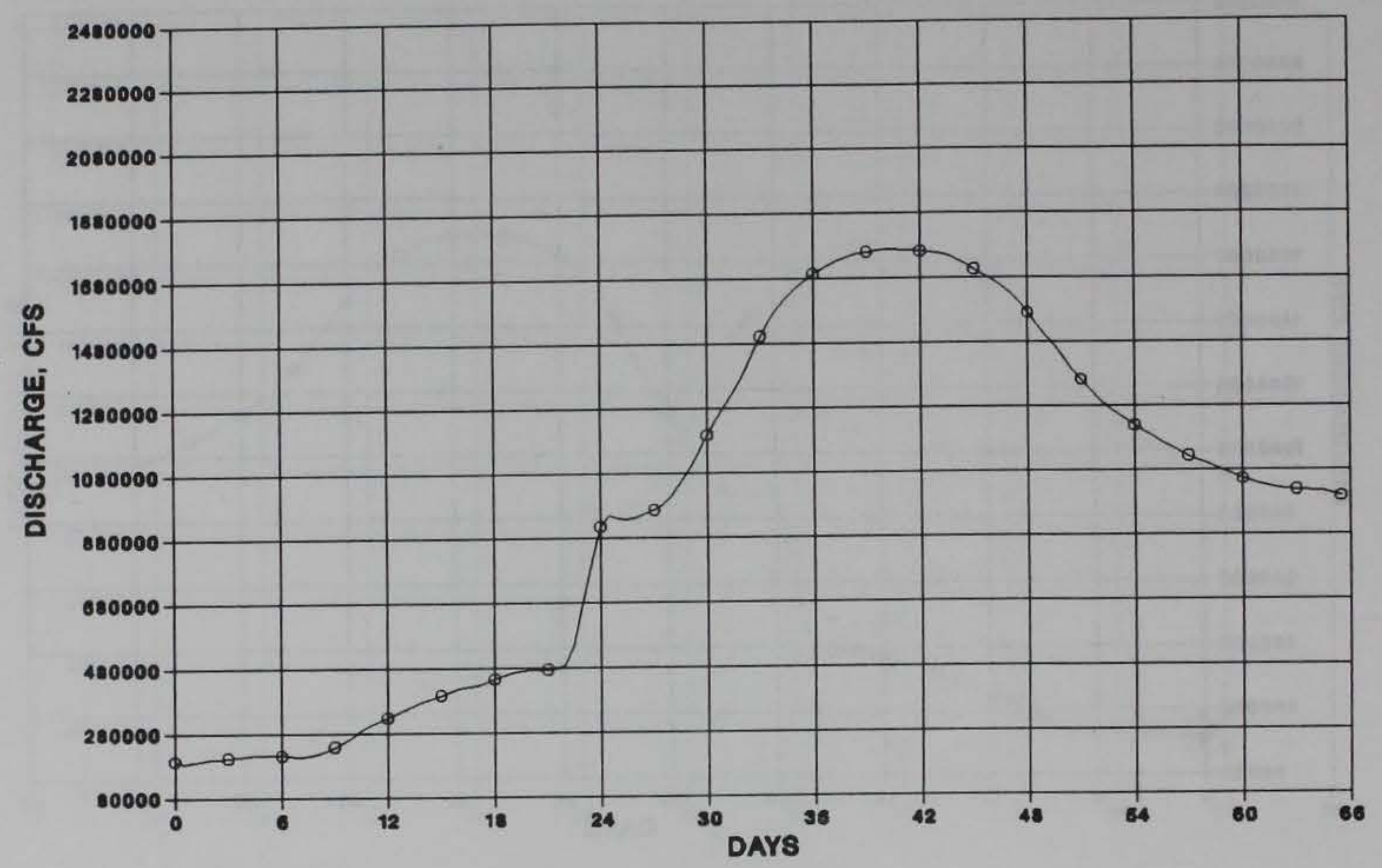

a. Discharge

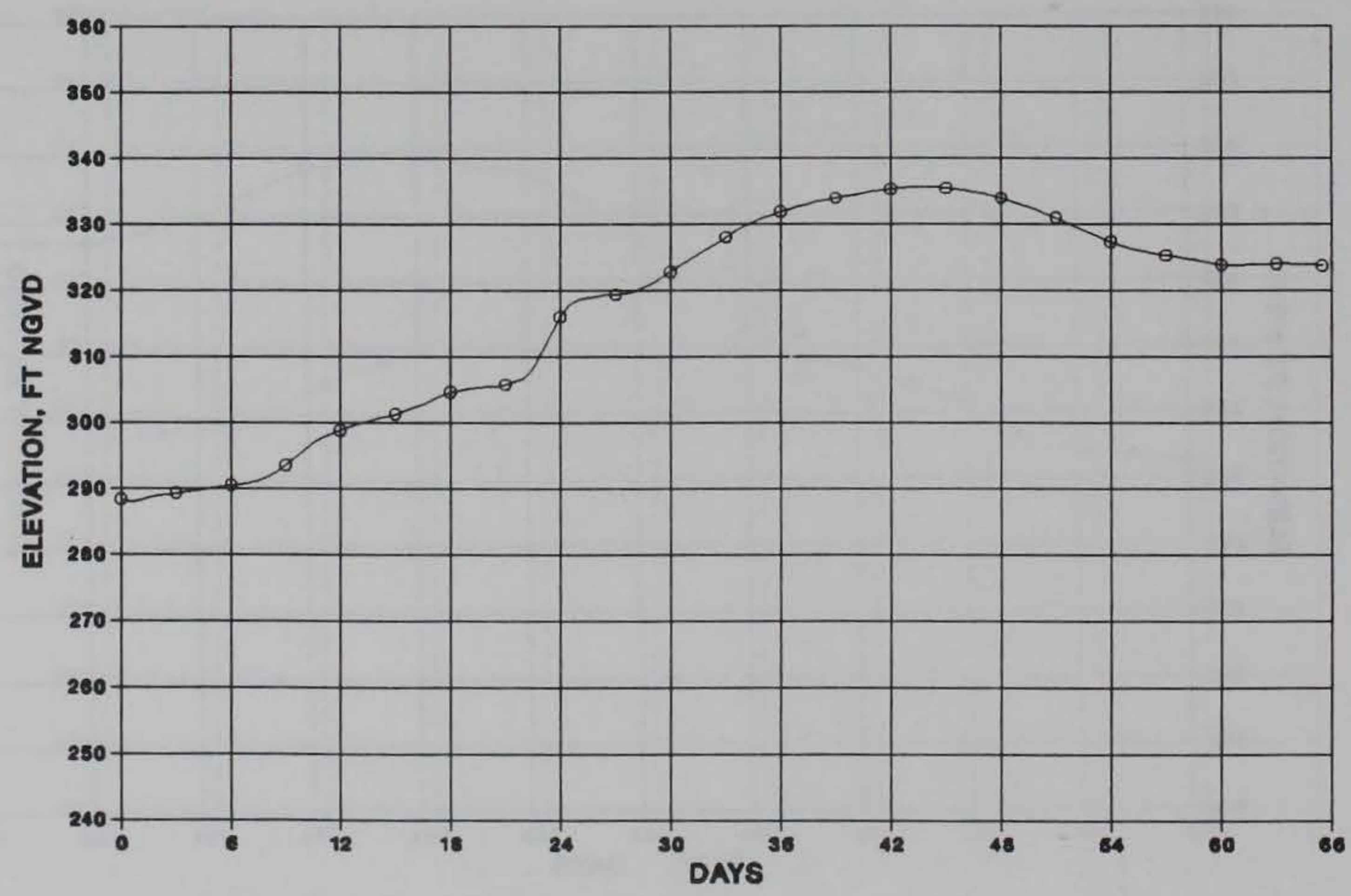

b. Elevation

Figure 103. Results at Cairo, IL, for Tellico PMF dam break 


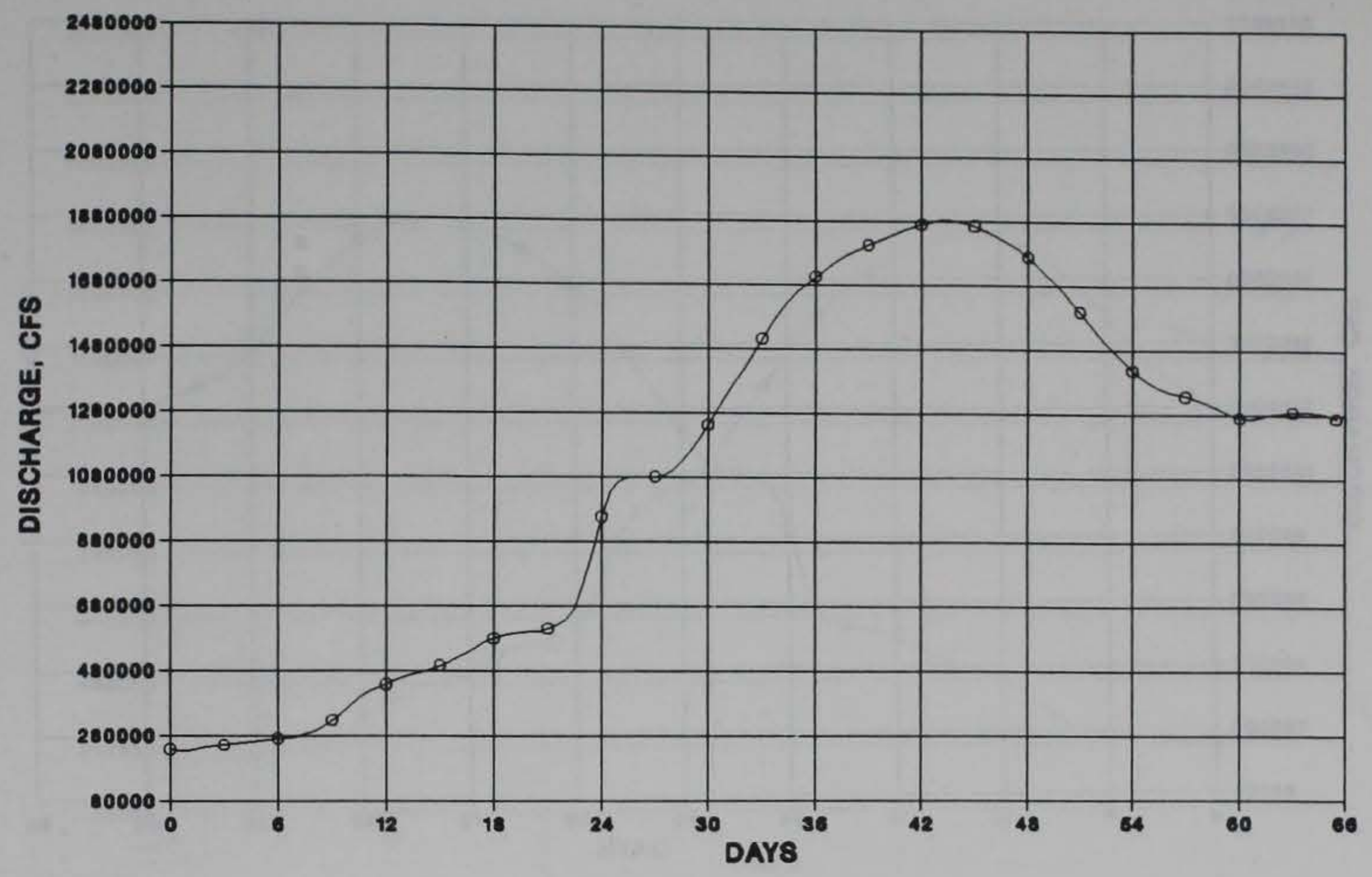

a. Discharge

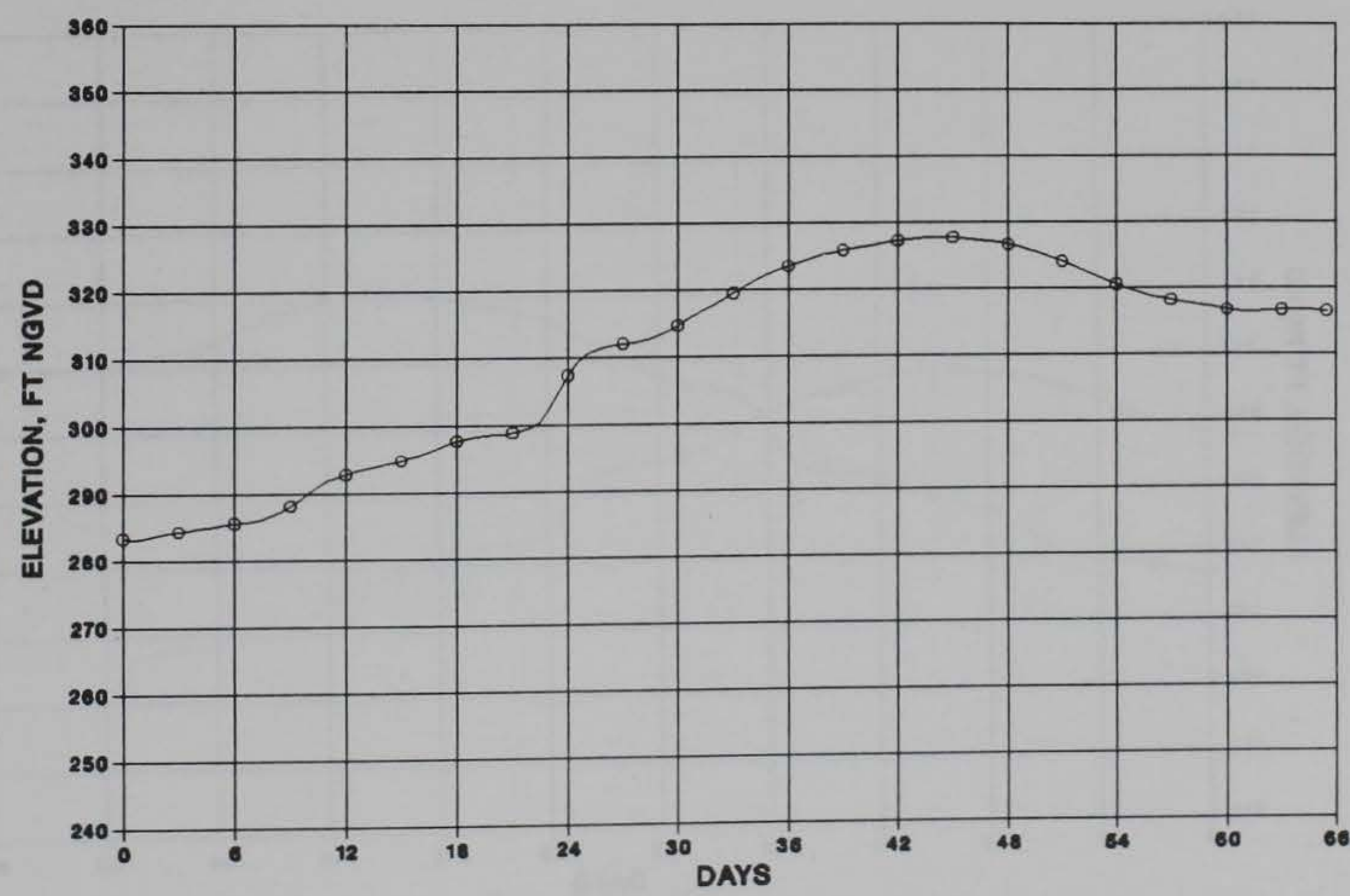

b. Elevation

Figure 104. Results at Columbus, KY, for Tellico PMF dam break 


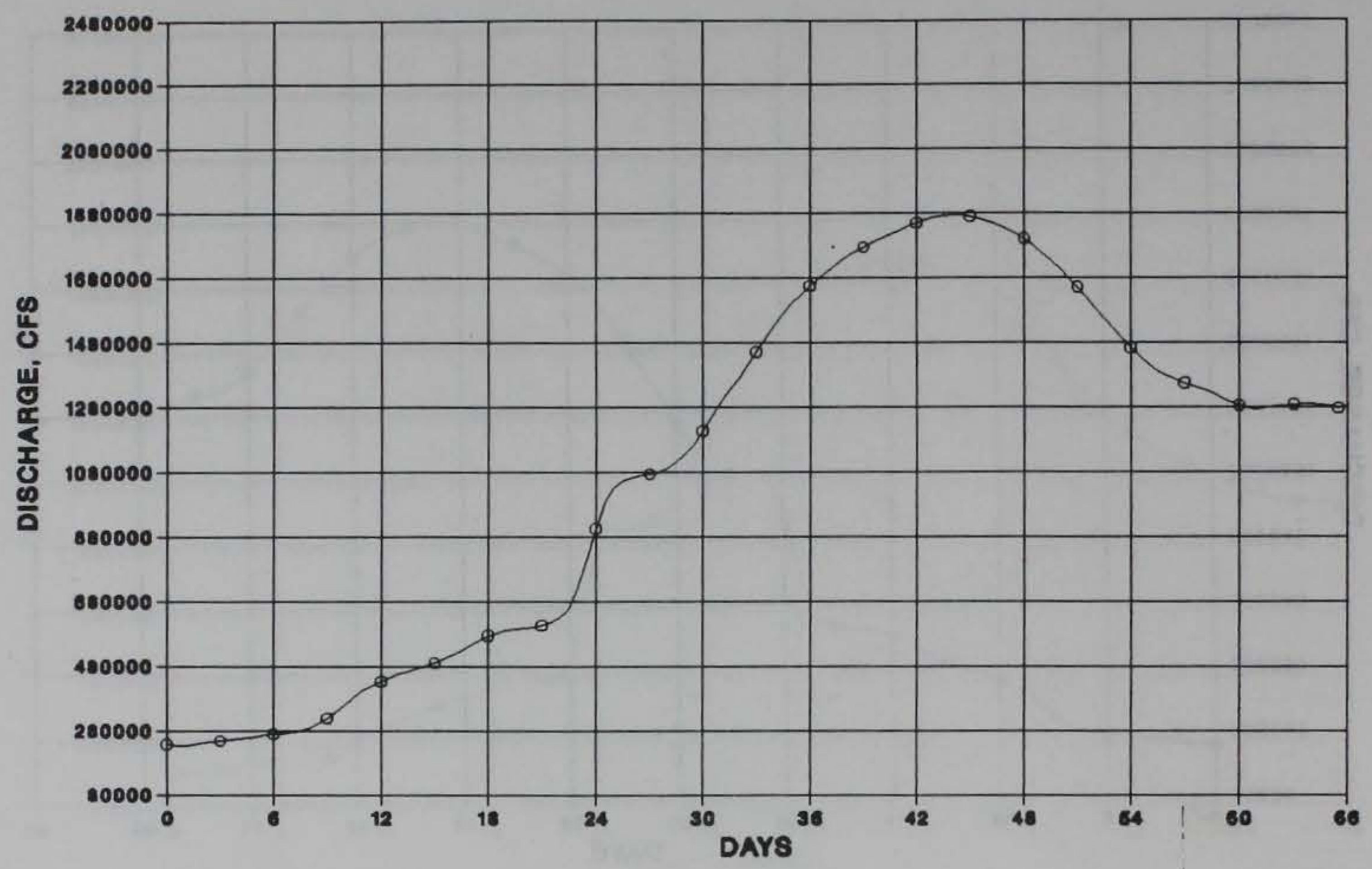

a. Discharge

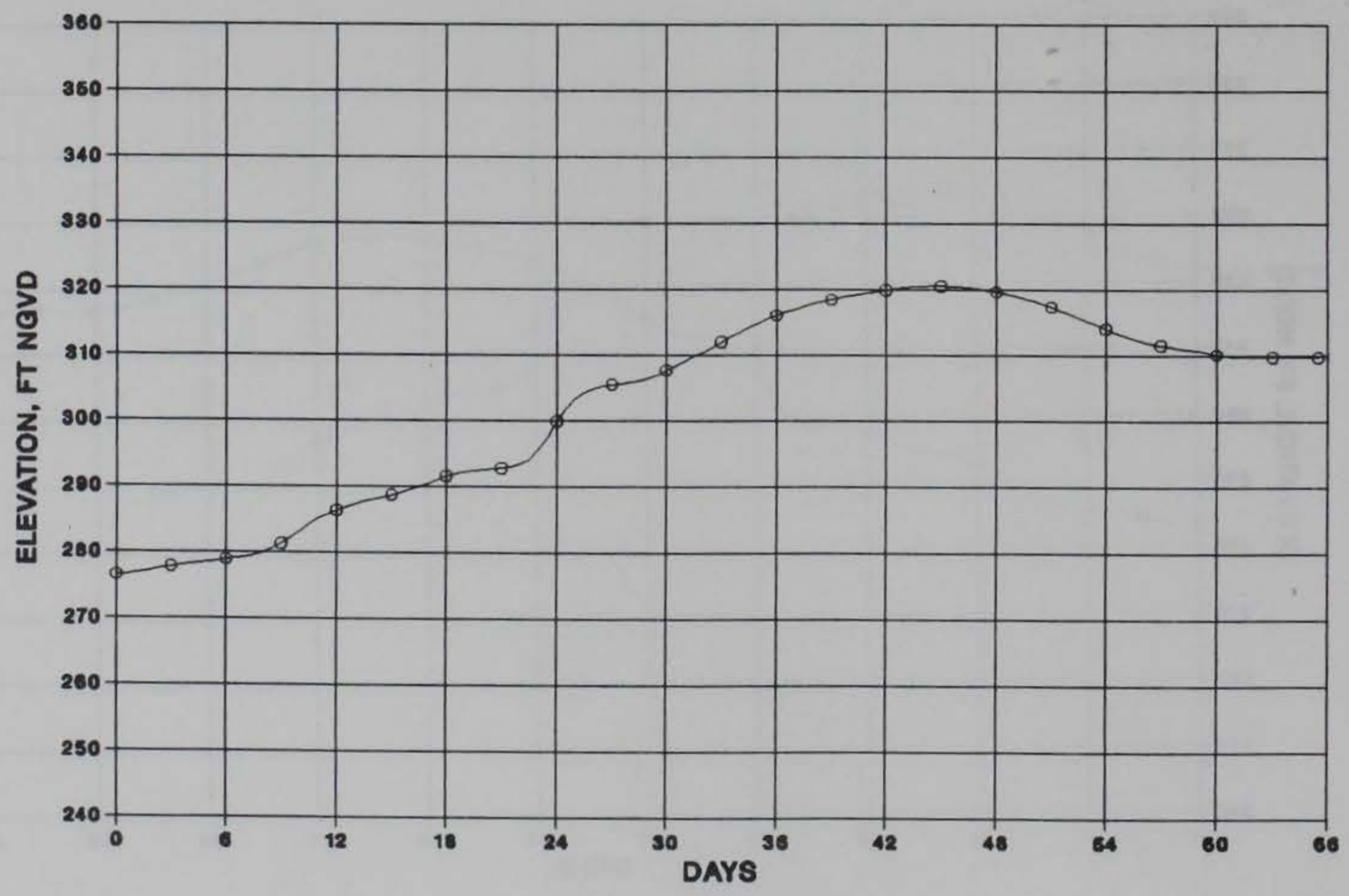

b. Elevation

Figure 105. Results at Hickman, KY, for Tellico PMF dam break 


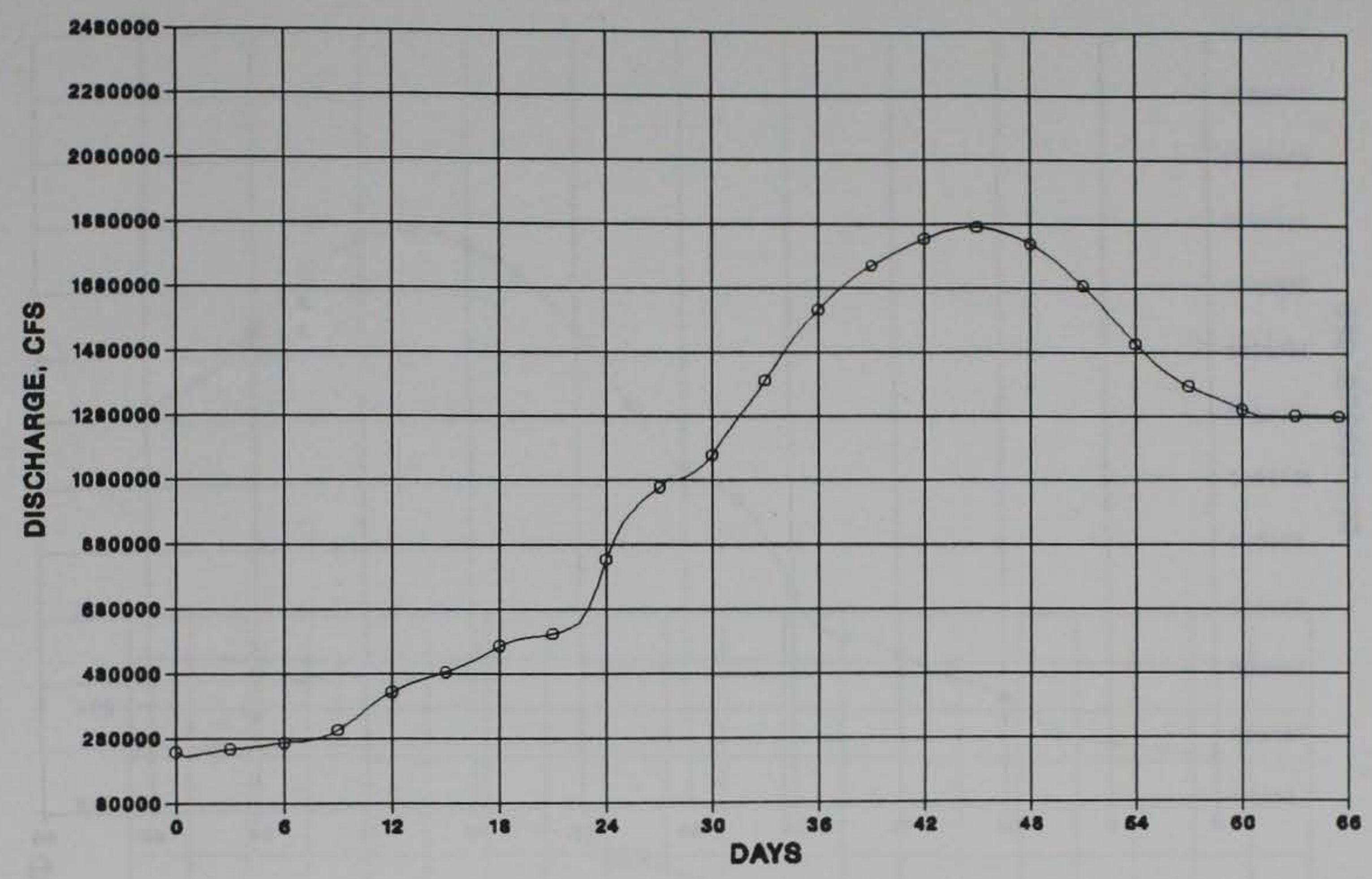

a. Discharge

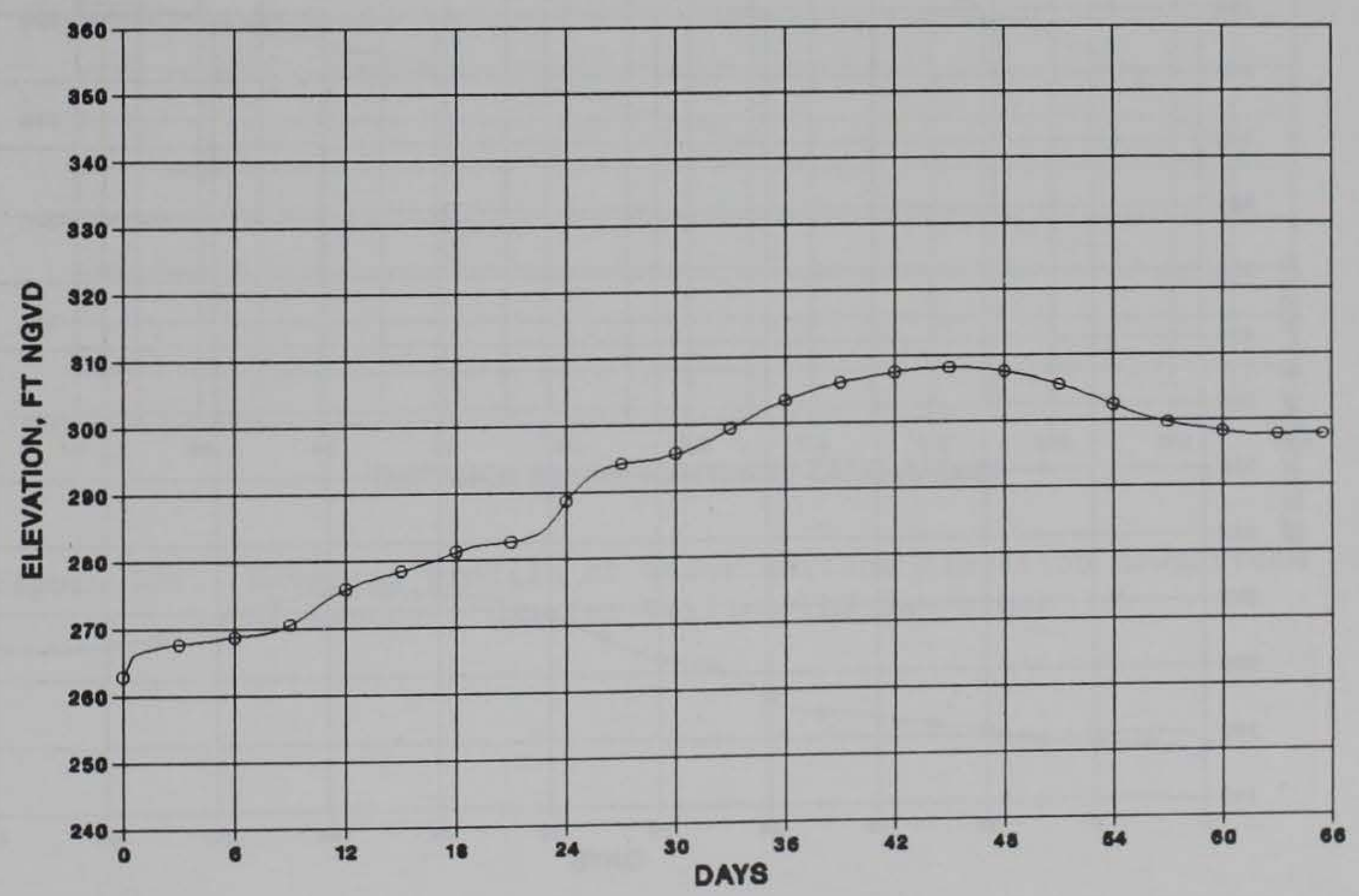

b. Elevation

Figure 106. Results at New Madrid, MO, for Tellico PMF dam break 


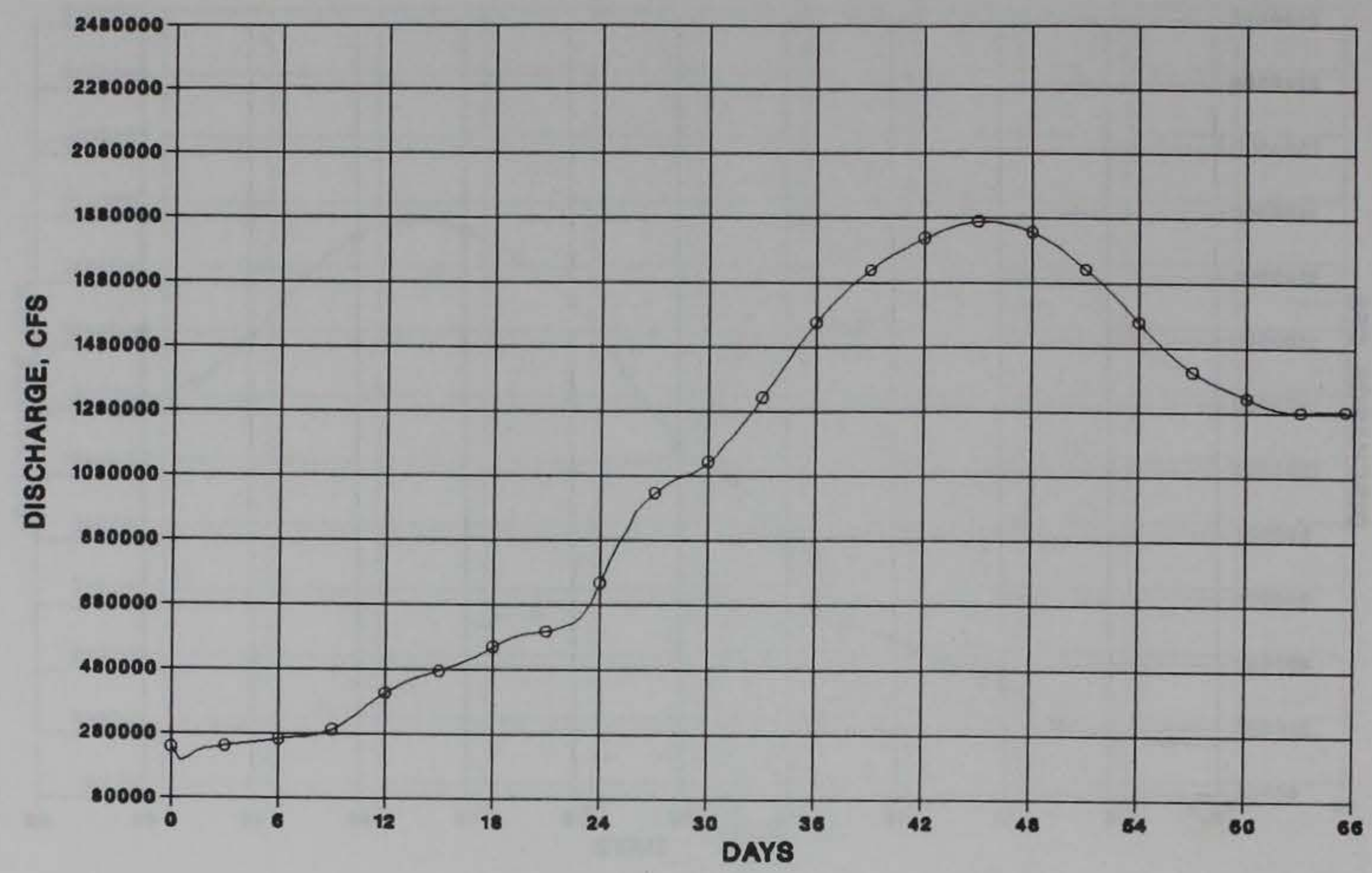

a. Discharge

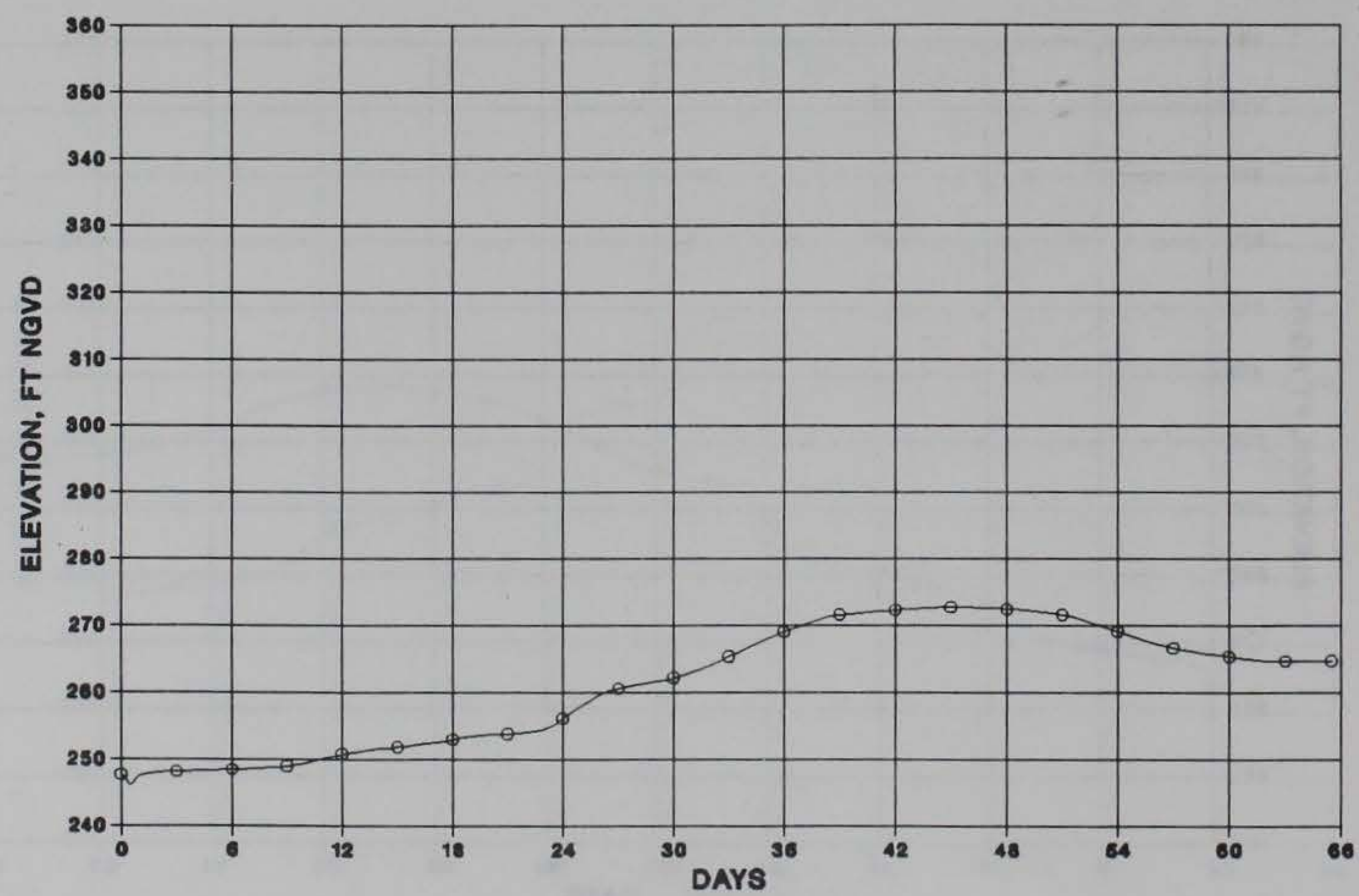

b. Elevation

Figure 107. Results at Caruthersville, MO, for Tellico PMF dam break 


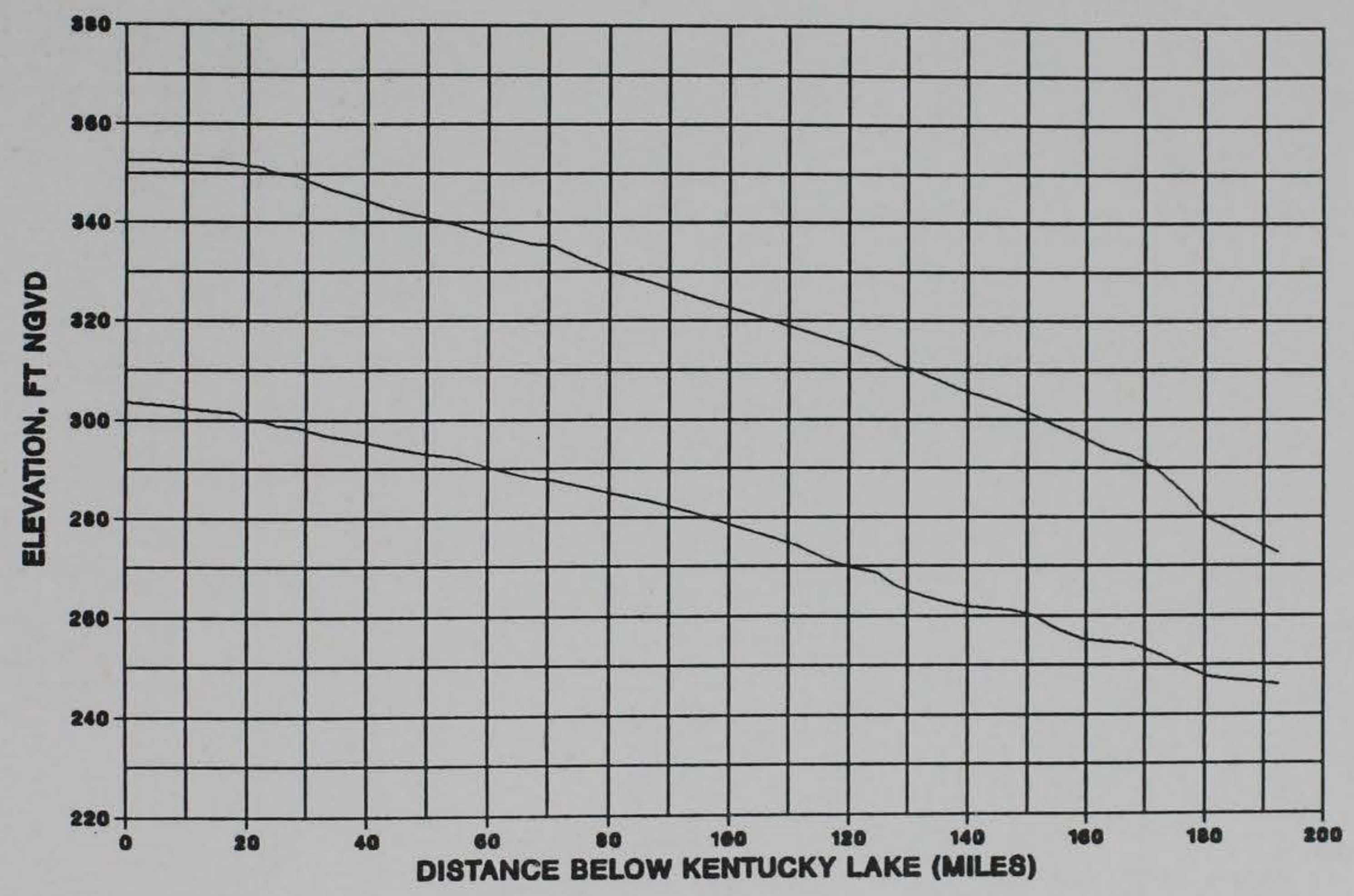

Figure 108. Envelope profile of water surface elevation downstream of Kentucky Dam for Tellico PMF dam break 